\title{
Dense Granular Fluids and the Granular Glass Transition
}

\author{
Dissertation zur Erlangung des \\ mathematisch-naturwissenschaftlichen Doktorgrades \\ „Doctor rerum naturalium“" \\ der Georg-August-Universität Göttingen
}

\author{
vorgelegt von \\ Wolf Till Kranz \\ aus Mainz
}

Göttingen 2011 
D 7

Referent: Prof. Dr. Annette Zippelius Korreferent: Prof. Dr. Reiner Kree

Tag der mündlichen Prüfung: 26. 9. 2011 


\section{Contents}

1 Collective Effects in Dense Fluids 1

1.1 Introduction . . . . . . . . . . . . . . . . . . . 1

1.2 Hard Spheres: Elastic and Inelastic Collisions . . . . . . . . . . . . 6

$1.2 .1 \quad$ Elastic Hard Spheres . . . . . . . . . . . . . . . . . . . . . 6

$1.2 .2 \quad$ Inelastic Hard Spheres . . . . . . . . . . . . . . . . . . 7

1.3 Dense Fluids . . . . . . . . . . . . . . . . . . . . . . . . . . . . 10

$1.3 .1 \quad$ Spatial Correlations . . . . . . . . . . . . . . 10

$1.3 .2 \quad$ Long-Time Tails . . . . . . . . . . . . . . . . . . . . 11

1.4 Supercooled Liquids and the Glass Transition . . . . . . . . . . . . 13

1.4 .1 Molecular Glass-Former . . . . . . . . . . . . . . . . . 13

1.4 .2 The Colloidal Glass Transition . . . . . . . . . . . . . . . . . . . 18

1.4 .3 The Granular Glass Transition . . . . . . . . . . . . . . . . . 19

1.5 Mode Coupling Theories . . . . . . . . . . . . . . . . . . 20

2 Microscopic Dynamics and Averages 23

2.1 Conservative Dynamics $\ldots \ldots \ldots \ldots$. . . . . . . . . . . . 23

2.2 Hard Spheres . . . . . . . . . . . . . . . . . . . . . . . . . . . . . . 27

2.3 Inelastic Hard Spheres . . . . . . . . . . . . . . . . . . . . . . . 28

2.4 Perspectives $\ldots \ldots \ldots \ldots \ldots \ldots$

$\begin{array}{lll}3 & \text { The Static Structure } & \mathbf{3 1}\end{array}$

$3.1 \quad$ Fluctuating Hydrodynamics . . . . . . . . . . . . . . . . . . . . . . 32

$3.1 .1 \quad$ Hydrodynamic Equations . . . . . . . . . . . . . . . . . 32

3.1 .2 Linearzation and Relaxation Rates . . . . . . . . . . . . . . . 34

$3.1 .3 \quad$ Fluctuating Hydrodynamics . . . . . . . . . . . . . . . . . 36

3.1 .4 Static Correlation Functions . . . . . . . . . . . . . . . . 37

3.2 Simulations . . . . . . . . . . . . . . . . . . . . . 40

3.2.1 $\quad$ Event Driven Molecular Dynamics and Volume Driving . . . 40

3.2 .2 Initial Conditions and Simulation Parameters . . . . . . . . . 41

$3.2 .3 \quad$ Measuring Correlation Functions . . . . . . . . . . . . . . 42

3.3 Results . . . . . . . . . . . . . . . . . . . . . . . . . . . . . . 43

3.3 .1 Static Correlation Functions . . . . . . . . . . . . . . . . 44

3.3 .2 Speed of Sound . . . . . . . . . . . . . . . . . 46 
3.3.3 Shear Viscosity and Sound Damping for Naive Volume Driving 47

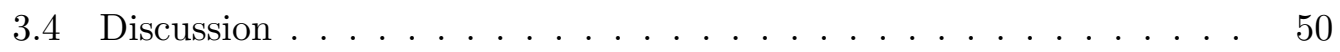

4 Mode Coupling Theories: An Overview 57

4.1 Memory Equations . . . . . . . . . . . . . . . . . . 57

4.1 .1 The Velocity Autocorrelation Function . . . . . . . . . . . 57

4.1 .2 The Coherent Scattering Function . . . . . . . . . . . 58

$4.1 .3 \quad$ Tagged Particle Dynamics . . . . . . . . . . . . . . . . . . 59

4.2 Back Scattering and Long-Time-Tails . . . . . . . . . . . . . . . . 61

4.3 The Coherent Scattering Function and the Glass Transition . . . . . 62

4.4 Coupling of the Tagged Particle to the Coherent Modes . . . . . . . 64

5 The Velocity Autocorrelation Function 67

5.1 Mode Coupling Theory for Elastic Hard Spheres . . . . . . . . . . . 67

5.2 Mode Coupling Theory for a Driven Granular Fluid . . . . . . . . . 70

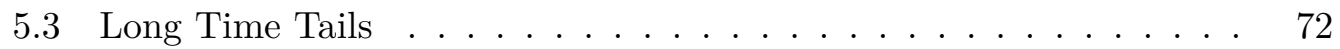

5.4 Discussion . . . . . . . . . . . . . . . . . . . . . . 73

6 Granular Mode Coupling Theory $\quad \mathbf{7 5}$

6.1 Mode-Coupling and the Glass Transition of Elastic Hard Spheres . . 75

6.2 The Granular Glass Transition . . . . . . . . . . . . . . . . . 78

$6.2 .1 \quad$ The Equation of Motion . . . . . . . . . . . . . . . . . 79

$6.2 .2 \quad$ Granular Mode Coupling Theory . . . . . . . . . . . . . . . . 80

6.3 Discussion . . . . . . . . . . . . . . . . . . . . . 82

6.4 Perspectives $\ldots \ldots \ldots \ldots \ldots \ldots$. . . . . . . . . . . . . . . . . 84

7 Tagged Particle Dynamics Close to the Glass Transition 87

7.1 Granular MCT for the Incoherent Scattering Function . . . . . . . . 87

7.2 Discussion $\ldots \ldots \ldots \ldots \ldots \ldots$

\begin{tabular}{|ll}
\hline A The Laplace Transform & 91
\end{tabular}

\begin{tabular}{ll}
\hline B Driving & 93
\end{tabular}

\begin{tabular}{ll}
\hline C Fluctuating Hydrodynamics & $\mathbf{9 5}$
\end{tabular}

C.1 Hydrodynamic Eigenvalues . . . . . . . . . . . . . . . . . . . . . . 95

C.2 $\quad$ Structure Factors from Fluctuating Hydrodynamics . . . . . . . . . . 96

\begin{tabular}{|lr}
\hline D Mode Coupling Diagrams & 97
\end{tabular}

\begin{tabular}{ll}
\hline E The Mori Identity & 99
\end{tabular} 
F Matrix Elements of the Liouville Operator 101

F.1 The Frequency Matrix . . . . . . . . . . . . . . . . . . . . . 101

F.2 The Vertices. . . . . . . . . . . . . . . . . . . . . . . . . . . 104

F.2.1 The Velocity Autocorrelation Function . . . . . . . . . . . . 104

F.2.2 The Incoherent Scattering Function . . . . . . . . . . . . 105

F.2.3 The Coherent Scattering Function … . . . . . . . . 106

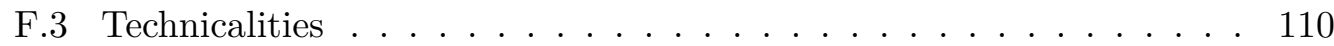





\section{List of Figures}

1.1 Examples of Granular Materials . . . . . . . . . . . . . . . . . . 2

1.2 Pitch Drop Experiment \& Angell Plot . . . . . . . . . . . . . . . . 3

1.3 Jamming Diagram . . . . . . . . . . . . . . . . . . . . 4

$1.4 \quad$ VACF for Elastic \& Inelastic Hard Spheres . . . . . . . . . . . . . 5

$1.5 \quad$ Static Structure Factor for Elastic \& Inelastic Hard Spheres . . . . . 6

$1.6 \quad$ Entropy of a Glass Former . . . . . . . . . . . . . . . . . . 13

1.7 Susceptibility Spectrum \& Intermediate Scattering Function of a

Lenard Jones Fluid . . . . . . . . . . . . . . . . . . . . . . . . 15

1.8 Mean Square Displacement for Molecular, Colloidal \& Granular Fluids 17

$1.9 \quad$ Granular Glass Transition \& Coherent Scattering Function . . . . . 20

$3.1 \quad$ Static Structure Factor $S(k)$, Naive Volume Driving $\ldots \ldots \ldots$. . . . . 45

$3.2 \quad$ Static Structure Factor $S(k)$, Local Driving . . . . . . . . . . . . . 46

$3.3 \quad$ Direct Correlation Function $n c(k)$, Naive Volume Driving $\ldots . . . . \quad 47$

$3.4 \quad$ Direct Correlation Function $n c(k)$, Local Driving $\ldots \ldots . . . . . . .49$

$3.5 \quad$ Longitudinal Current Correlator $S_{\ell \ell}(k)$, Naive Volume Driving . . . 50

$3.6 \quad$ Longitudinal Current Correlator $S_{\ell \ell}(k)$, Local Driving $\ldots . . . . . .551$

3.7 Transverse Current Correlator $S_{\perp \perp}(k)$, Naive Volume Driving . . . . 52

3.8 Transverse Current Correlator $S_{\perp \perp}(k)$, Local Driving. . . . . . . . . . 53

3.9 Density-Temperature Cross-Correlation Function $S_{n T}(k) \ldots \ldots$

3.10 Temperature Correlator $S_{T T}(k) \ldots \ldots \ldots \ldots \ldots$

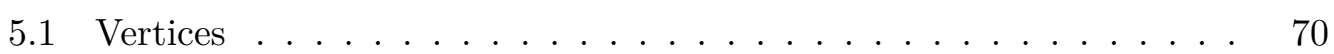

$6.1 \quad$ Speed of Sound $\ldots \ldots \ldots \ldots$. . . . . . . . . . . . . . . . 80

6.2 Granular Jamming Diagram \& EA Order Parameter . . . . . . . . . 83

6.3 Critical Exponents $\ldots \ldots \ldots \ldots \ldots$. . . . . . . . . . . . . . 84

7.1 Incoherent Scattering Function \& Lamb-Mößbaur Factor . . . . . . . 89

7.2 Mean Square Displacement \& Localization Length . . . . . . . . . . 90 



\section{Collective Effects in Dense Fluids}

\subsection{Introduction}

Letting sand flow through your fingers it certainly looks and feels like a liquid. Nevertheless, you can walk on the sandy surface of a desert. Supporting the stresses imposed by your weight, sand displays properties of a solid body. The formation of stable sand piles by slowly pouring sand on the ground (e.g. in an hour glass, Fig. 1.1 also demonstrates that sand, or more generally, granular matter, can jam and pass from a fluid state into an amorphous solid state. Adding some water makes it possible to build highly detailed sand castles [1].1 Particularly interesting is the tendency of granular systems to spontaneously form patterns [4 6].

The ability for granular matter to flow through chutes and hoppers also makes it, besides water, one of the most important form of matter handled by the industry [11]. This works well until a hopper jams and the granular material turns into a disordered solid. Naturally, the behavior of granular matter is also important for geophysics, e.g. to describe avalanches and debris flow [12] or the natural formation of ordered stone stripes [13]. Astoundingly, some animals have figured out how to swim in sand. Their technique has been understood only recently [14, 15]. As far as the early stages of planet formation [16] or the dynamics of planetary rings [17, 18] are concerned (the rings of Saturn being the most prominent [19]), the field of astrophysics also benefits from a detailed understanding of granular matter. In astrophysical conditions, the concentration of particles is so low that one can speak of a granular gas. At this point it appears that, depending on circumstances, one can have granular solids, liquids and gases [20]. All these granular "phases" are under active investigation. Some of the established results can be found in reviews by Jaeger et al. [21, 22, Rajchenbach [23] and Herminghaus [24].

Another class of amorphous solids that we are familiar with is glass. Although there is no clear cut definition and no consensus of what exactly defines a glass, let me for the moment define it as a non-crystalline, i.e. disordered solid formed by rapidly cooling a melt. Window glass (soda-lime glass) is the most well-known example but glassy materials are also common among food (including cotton candy) and thus glasses are highly relevant for the food industry [25, 26]. Even living cells can show aspects of glassy behavior [27]. The last two examples belong to the class

\footnotetext{
${ }^{1}$ Although they are not indestructible [2, they are remarkably easy to build 3 .
} 

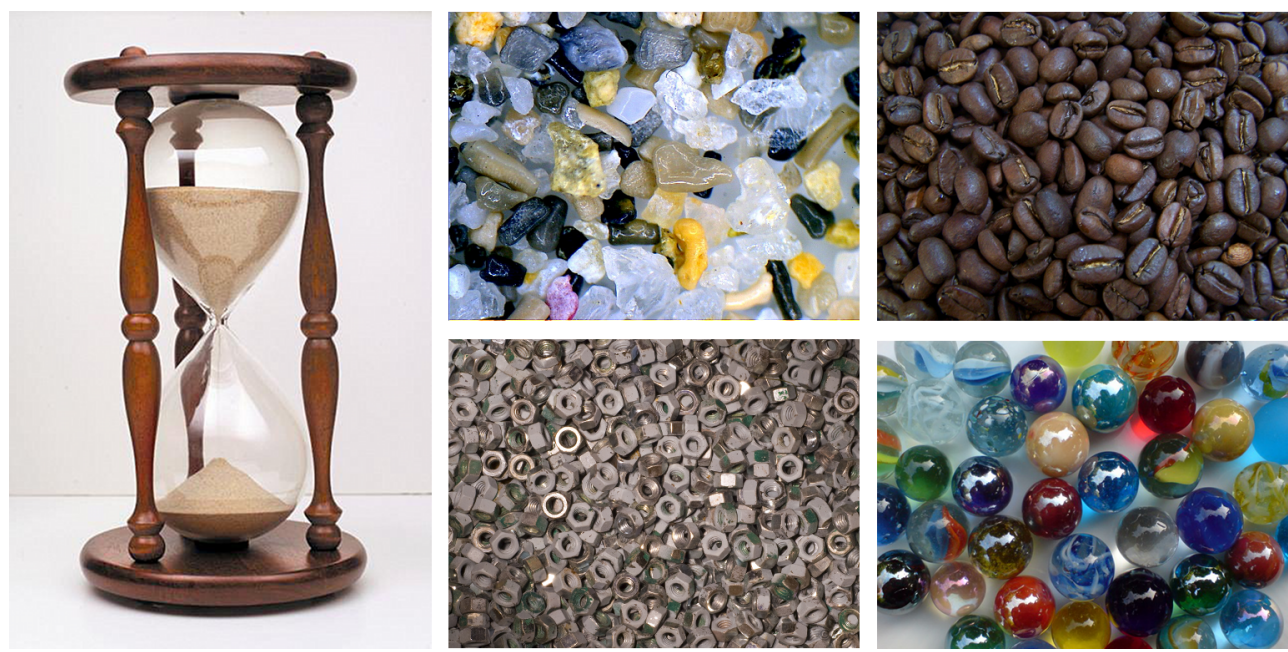

Figure 1.1: Left: A running hour glass contains sand both in the fluid and in the solid, jammed state. Incidentally, this picture also shows a strong glass former, silica glass. Picture courtesy Labormikro [7]. Right: A few examples of granular particles. Sand from the Baia di Porto Frailis, Sardinia; Picture courtesy Labormikro [8]. Roasted coffee beans; Picture courtesy dominotic [9]. Painted steel nuts; Picture courtesy Ric Martin [10. Glass marbles; Picture courtesy A. von der Heydt.

of soft glassy materials [28] which are formed by complex, non-Newtonian fluids. For the importance of amorphous solids in material science, see e.g. the review by Greer on metallic glasses [29].

The standard technique to produce a glass is to start with a fluid or melt and rapidly cool it below its melting temperature. Depending on the material, freezing and crystallization may thereby be avoided. The viscosity of this supercooled melt then rises quickly upon further lowering the temperature until the system reaches the glass transition, conventionally defined as the temperature $T_{g}$ where the viscosity reaches $10^{13} \mathrm{P}$ (see Fig. 1.2). This is extremely viscous in human terms if one keeps in mind that the pitch drop experiment [30] (see Fig. 1.2) is reported to display a viscosity of approximately $10^{7}-10^{9} \mathrm{P}$.

The glass itself is generally regarded to be out of equilibrium. Its properties then depend on the time one waits after the glass has formed, a phenomenon called aging [33, 34]. A completely satisfactory theoretical description of the glassy state is still missing.2 The question of how and exactly where the glass transition of water takes place is, for example, still highly controversial [36], although there is evidence that most of the water in the universe is in its amorphous form [37.

\footnotetext{
${ }^{2}$ The journal Science even ranked the question "What is the nature of the glassy state?" among 100 important scientific questions that are not answered yet 35.
} 

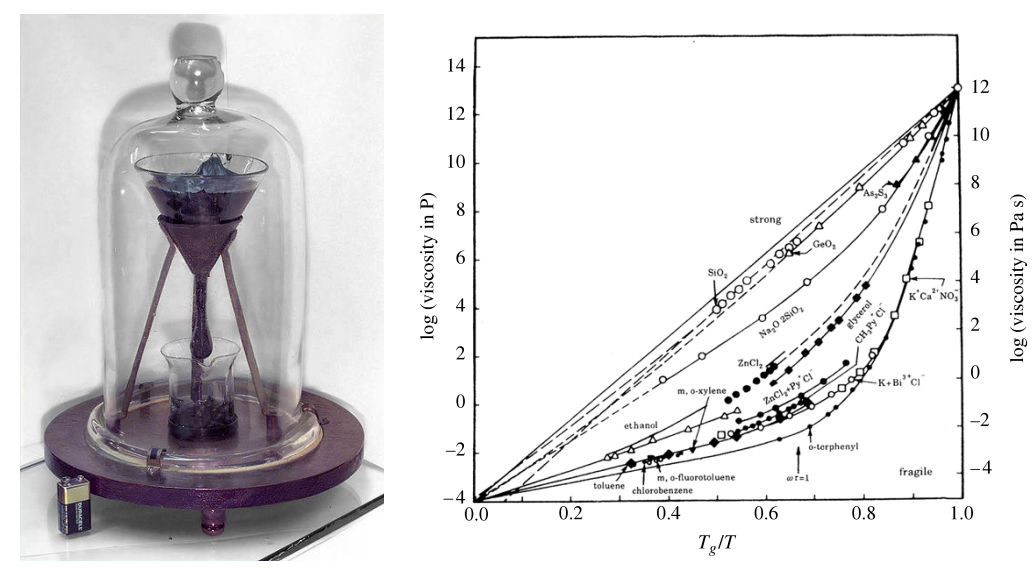

Figure 1.2: Left: The pitch drop expriment [30, 31, featuring an extremely viscous fluid. Each drop takes about ten years to fall. Right: Angell plot of the viscosity, $\eta$, as a function of inverse temperature, $T_{g} / T$, where the glass transition temperature, $T_{g}$, is defined as $\eta\left(T_{g}\right) \equiv 10^{13} \mathrm{P}$. Reprinted from Ref. [32], Copyright (2009), with permission from Elsevier.

At this point we have seen two ways to generate an amorphous solid from a fluid, in the granular case one increases the density of a flow until it jams and one gets a random close packed solid, and in the case of a melt one supercools it until one gets an amorphous glass. It turns out that in both cases one can destroy or melt the solid by loading or shearing it. This led to the idea of fragile matter [38] and the jamming diagram [39]. The conjecture is that amorphous solids can be described in a unified way in terms of the variables density, $\varphi$, temperature, $T$, and shear stress, $\sigma$ (see Fig. 1.3). A lot of work has been devoted to the jamming diagram in recent years (see Ref. [40] and references therein) but the precise form of this unified description has still to be worked out.

The choice of axes on the jamming diagram implies that the temperature, $T$, and the shear rate, $\sigma$, are understood as independent variables. Consequently, systems supposedly described by the jamming diagram must implicitly contain either a heat bath or an energy sink to balance the energy input due to shearing. For systems with an energy sink, one has to consider nonequilibrium jamming- or glass transitions. For nonequilibrium granular fluids, experimental signatures of a glass transition have indeed been recently found [41,46].

From a theoretical point of view, molecular fluids are most easily understood in the limit of a very dilute gas. On the level of kinetic theory, they are well described by the Boltzmann equation. To get a continuum description, there are carefully worked out procedures to derive hydrodynamic equations from this starting point [48]. The 


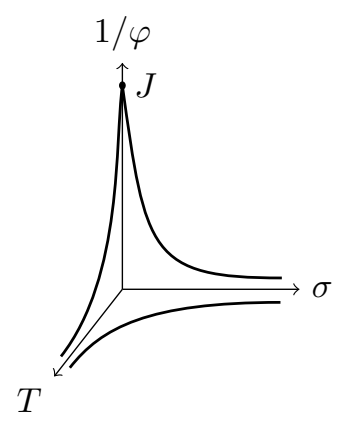

Figure 1.3: The jamming diagram as proposed by O'Hern et al. [47. The region of low temperature $T$, low shear stress $\sigma$ and high volume fraction $\varphi$ around the origin is in the jammed state while outside of this region the system shows fluid behavior. The jamming density is marked as point $J$.

Navier-Stokes equations that describe an isothermal fluid are the most important instance [49] of such a continuum description. A hydrodynamic description has also proven to be successful for granular gases, although it is actually more common to encounter situations that fall outside the range of validity of hydrodynamics [50, 51]. Using granular hydrodynamic equations allowed, for example, the discussion of the stability of granular fluids and explanations of the clustering instability of a freely cooling granular gas [52, 53], or analysis of possible mechanisms leading to the brazil nut effect [54 56]. More can be found in the reviews by Kadanoff [57] and Goldhirsch [58].

Unexpectedly, it was found in computer simulations by Alder and Wainwright [59, 60], that already at moderate densities, equilibrium fluids showed dynamic phenomena that are not explained by the most simple hydrodynamic description. In particular, the velocity autocorrelation function, $\psi(t)$, was expected to decay exponentially, $\psi(t) \propto e^{-t / \tau}$, on macroscopic time scales, but was actually found to exhibit long-time tails, $\psi(t) \propto t^{-d / 2}$, in $d$ dimensions (see Fig. 1.4). Shortly afterward, these long-time tails were also found for other correlation functions [61 and they were traced back to the effect of ring collisions, i.e. loops in the sequence of colliding particles [62, 63]. While the long-time tails are interesting in their own right, the correlated interactions also ultimately prevent a description of a perturbed fluid at finite density as an expansion around the unperturbed state [64, 65].

A second qualitative feature of the velocity autocorrelation function at higher densities is that it acquires negative values for intermediate times. This signals the onset of backscattering, i.e., the tagged particle becomes increasingly trapped by its neighbors and is reflected from them. In recent years, observations of long time tails and backscattering in simulations and experiments of various granular systems have been reported [42, 66, 67] (see Fig. 1.4).

Crystalline order can be detected in neutron- or light scattering experiments through the appearance of well-defined Bragg peaks [68]. Measurements of the scattering 

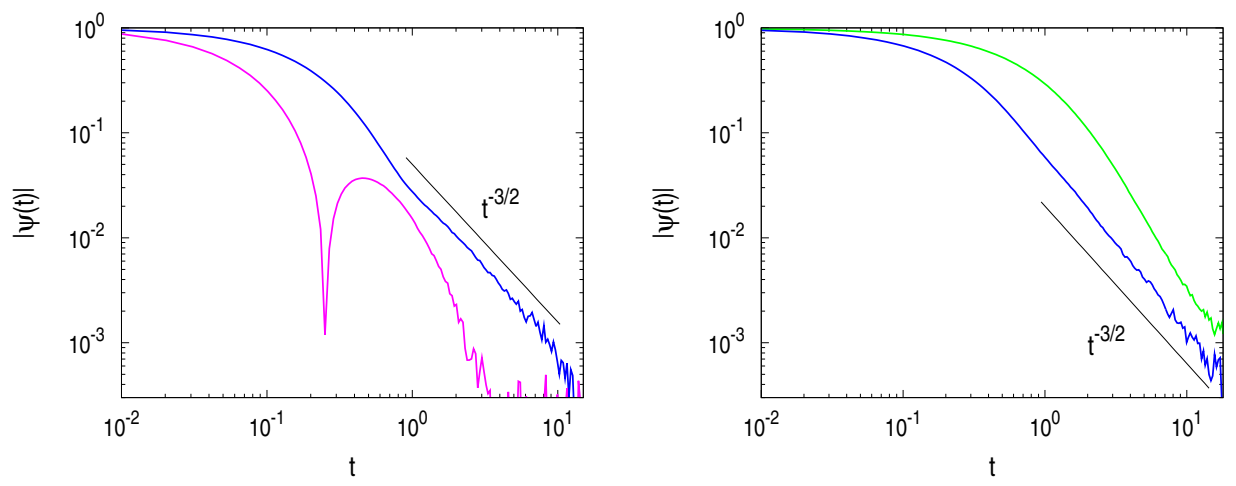

Figure 1.4: Left: The velocity autocorrelation function, $|\psi(t)|$, of an elastic hard sphere fluid for volume fraction $\varphi=0.35$ (blue) and $\varphi=0.5$ (magenta). Note the double logarithmic scaling. Right: Long time tails of the velocity autocorrelation function in a driven granular fluid with a coefficient of restitution $\epsilon=0.7$ for volume fraction $\varphi=0.2$ (green) and $\varphi=0.35$ (blue). Data courtesy A. Fiege.

function, $S(\boldsymbol{q})$, for isotropic fluids show no such peaks in accordance with the absence of long range order. At finite densities, the organization of the particles into shells of nearest neighbors, next nearest neighbors, and so on, appears instead as radial modulations of $S(\boldsymbol{q})$ [69] (see Fig. 1.5). This makes it easy to distinguish fluid and crystal phases based on the scattering function. The scattering function of a glass, on the other hand, does not possess any qualitative features that would differentiate it from that of a fluid [70].

The small wave number limit, $\lim _{q \rightarrow 0} S(q)$, is related to the magnitude of macroscopic density fluctuations and, for equilibrium fluids, to the compressibility [69]. A divergence of $S(q \rightarrow 0)$ and consequently of the compressibility is one of the signatures of a liquid at its critical point [71. An analogous divergence of $S(q)$ for nonequilibrium systems that may occur in extended regions of the parameter space has been termed self-organized criticality [72. Depending on the method of fluidization, such a low $q$ divergence may also be found in granular systems [73, 74] (see Fig. 1.5).

In the following sections I will discuss some of the phenomena addressed above in more detail. In particular, I will state the main results I obtained in the course of my work. The derivation of these results will be given in the subsequent chapters of this thesis (chapters 2 27). Technical derivations are further described in the appendices. 

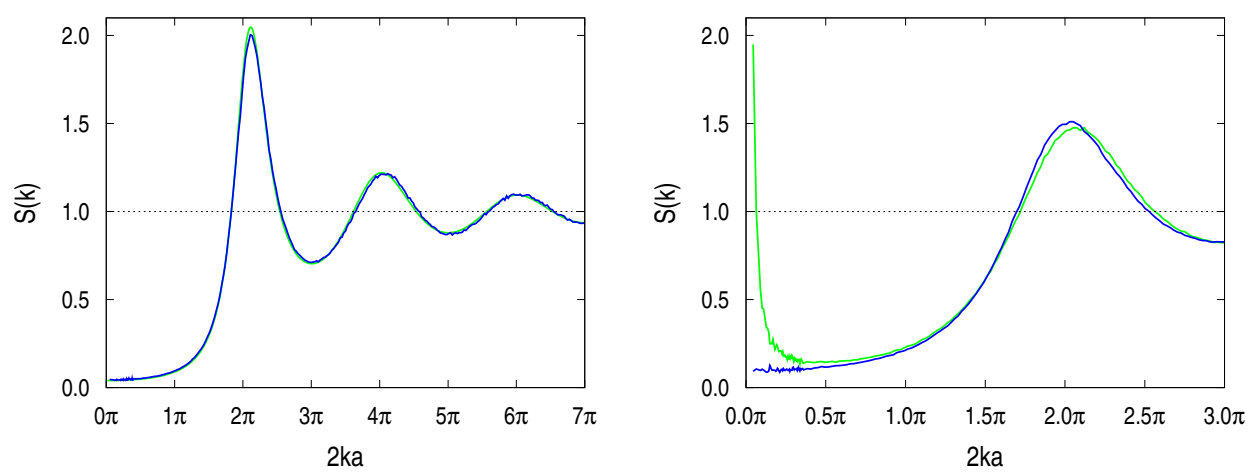

Figure 1.5: Left: Static structure factor, $S(k)$, for an equilibrium hard sphere fluid at $\varphi=0.4$ from an event driven simulation (blue) compared to the corresponding Percus-Yevick analytical approximation (green). Right: Static structure factor of a randomly driven granular fluid at a density $\varphi=0.3$ with (blue) and without (green) local momentum conservation.

\subsection{Hard Spheres: Elastic and Inelastic Collisions}

\subsubsection{Elastic Hard Spheres}

To describe the interactions of the atoms of a noble gas at room temperature, fortunately, one does not necessarily have to solve a quantum mechanical problem. Instead one can use classical potentials, like the Lennard-Jones potential. For some effects even this is too much detail and it suffices to neglect the attractive part of the potential and to approximate the repulsive part by an infinitely steep potential,

$$
V(r)=\left\{\begin{array}{r}
0 \text { if } r>2 a \\
\infty \text { if } r \leq 2 a
\end{array}\right.
$$

where $a$ is the sphere radius [69]. Another class of materials that is well described by hard spheres are colloids, i.e. suspensions of $\mu \mathrm{m}$-sized spherical particles in a solvent [75]. Due to their small size, single colloidal particles undergo random motion. Although hydrodynamic interactions between the colloidal particles may become important for dense suspensions, Brownian dynamics is usually assumed to remain a good approximation.

The distance dependence of the hard sphere interaction sets a length scale but there is no energy scale. Consequently, changing energy or temperature of a hard sphere fluid simply rescales the time scale (the particles move faster on average if the temperature is higher) but has no qualitative influence on the behavior of the system. Every nonzero temperature is equivalent. A related artifact of the hard sphere model (if regarded as an approximation to a short ranged potential) is the 
vanishing collision time. Collisions are necessarily instantaneous. This implies that the dynamics is determined by a sequence of two-particle collisions as the probability of $K$-particle collisions is zero for $K \geq 3$ and all initial conditions that are free of particle contacts.

With the temperature being an irrelevant control parameter, the behavior of hard sphere fluids is controlled by the number density, $n$, only. It is convenient to report densities in terms of the dimensionless volume fraction $\varphi:=4 \pi n a^{3} / 3$. The latter can never exceed the close packed value, $\varphi_{c p}=\pi / \sqrt{18} \approx 0.740$, valid for a fcc or hcp crystal. This has been assumed for a long time [76] but the recent proof by Hales [77] is still being cross checked.

Surprisingly at first sight, the hard sphere fluid still undergoes a first order crystallization transition to a fcc crystal at high densities [78]. As there is no interaction energy, the transition has to be driven by entropy alone. The intuitive argument is, that the loss in entropy due to the ordered structure of the crystal is overcompensated above the freezing density by the entropy gain due to the increased free volume ${ }^{3}$. Such a hard sphere phase transition is actually found in computer simulations (see, e.g., Ref. [80]) with a broad coexistence region between $\varphi_{f} \approx 0.494$ and $\varphi_{m} \approx 0.545$. While several theoretical approaches are able to reproduce the transition and even approximate the transition densities, they are still plagued by questions of convergence [78]. The question whether there is a crystallization transition for hard disks in two dimensions is even more difficult [81].

In the ordered, crystalline configuration, the packing density is bounded by the close packed value $\varphi_{\text {cp }}$. If, instead, a loose, athermal $(T=0)$ assembly of smooth spheres is compressed quasistatically, one finds that the density will not exceed the random close packed density $\varphi_{r c p} \approx 0.64[82$.

\subsubsection{Inelastic Hard Spheres}

Trying to describe the interactions of granular particles, one immediately realizes that they are extremely complicated objects in reality. First of all, they never come as a monodisperse system but, taking sand as an example, every grain is irregularly shaped and different from every other grain (see Fig. 1.1). Depending on the specific way two particles collide, they may dissipate different amounts of energy in a combination of elastic (see below) and plastic deformations [83, 84. Moreover, off-center collisions will let the particles rotate around their center of mass.

Various idealizations are commonly employed, both for the particle shape as well as for the interactions. While simulations can handle a limited amount of irregularity like (rounded) polyhedra [85, 86] or agglomerations of spheres [87, most studies,

\footnotetext{
${ }^{3}$ Free volume is the volume that is available to particle movement. Not to be confused with the volume fraction [79].
} 
both experimental and theoretical, consider spheres for simplicity. Exceptions include studies on inelastic needles [88, 89] and capped rectangles [90].

Hertz [91] studied the collision of two smooth elastic spheres already in 1882 . Later, attempts were made to describe dissipative effects on the level of continuum theories. The excitation of vibrations [92 94] and viscoelastic effects [95 97] have been considered. Especially for simulations, an interaction model based on an effective description via Hookian springs and viscous dampers is often used. It had first been proposed by Cundall and Strack [98. A more thorough discussion of various collision models can be found in Ref. [94.

The most idealized system of granular matter that still retains the properties that the particles are dissipative and of finite size is to model them as monodisperse smooth hard spheres with mass $m$ and radius $a$. Dissipation is parametrized by a constant coefficient of normal restitution, $\epsilon \in[0,1]$ (see, e.g., Refs. [99, 100]). The coefficient of restitution extends the law of reflection,

$$
\hat{\boldsymbol{r}}_{12} \cdot \boldsymbol{v}_{12}^{\prime}=-\epsilon \hat{\boldsymbol{r}}_{12} \cdot \boldsymbol{v}_{12}
$$

which relates the relative velocity immediately before the collision, $\boldsymbol{v}_{12}=\boldsymbol{v}_{1}-\boldsymbol{v}_{2}$, to the relative velocity, $\boldsymbol{v}_{12}^{\prime}$, immediately after the collision. Here, $\hat{\boldsymbol{r}}_{12}$ is the unit vector pointing from the center of one sphere to the center of the other. Together with the condition of smoothness, $\hat{\boldsymbol{r}}_{12} \times \boldsymbol{v}_{12}^{\prime}=\hat{\boldsymbol{r}}_{12} \times \boldsymbol{v}_{12}$, and the conservation law of momentum, this uniquely determines the postcollisional velocities in terms of the precollisional ones,

$$
\boldsymbol{v}_{1,2}^{\prime}=\boldsymbol{v}_{1,2} \mp \frac{1+\epsilon}{2}\left(\hat{\boldsymbol{r}}_{12} \cdot \boldsymbol{v}_{12}\right) \hat{\boldsymbol{r}}_{12},
$$

This is precisely the model I will use in the rest of this thesis. For experimental measurements of the coefficient of restitution see, e.g., Ref. [101].

In analogy to the kinetic definition of the temperature of a molecular gas, one defines the granular (kinetic) temperature, $T=m\left\langle\boldsymbol{v}^{2}\right\rangle / d$, as the mean kinetic energy per particle. The most basic situation to describe is the free cooling of a granular fluid that has been prepared in a homogeneous state of temperature $T_{0}$. Assuming that the system remains homogeneous, an explicit equation for the time evolution of the temperature, $T(t)$, can be derived, Haff's law [99],

$$
T(t)=\frac{T_{0}}{\left(1+\omega_{0} t / 2 \tau_{0}\right)^{2}},
$$

where $\omega_{0} \equiv \omega\left(T_{0}\right)$ is the initial collision frequency and $\tau_{0}=d /\left(1-\epsilon^{2}\right)$ is the relaxation time scale if we measure time as the number of collisions $\tau, T(\tau)=T_{0} \exp \left(-\tau / \tau_{0}\right)$. A stability analysis of the hydrodynamic equations derived for the so called homogeneous cooling state predicts that it is actually unstable toward a inhomogeneous, clustered state if the system exceeds a critical size, $L_{c}(\varphi, \epsilon)$, depending on the density and the coefficient of restitution [52, 53]. 
To maintain a granular system in a homogeneous, fluidized state, it has to be driven continuously to replenish the kinetic energy that gets dissipated in particle collisions. While in practice, gravity is probably the most important driving force, experimentalists have devised quite a few methods of fluidisation. The list includes shaking [102], electrostatic [103, 104] or magnetic [104, 105] excitation and fluidisation by air [106, 107] or water [108].

On the level of a theoretical description, shaking imposes boundary conditions on the system and thus destroys homogeneity. Fluidisation through the interaction with a (turbulent) host fluid (be it a gas or a liquid) will in general be more homogeneous. Unfortunately, hydrodynamic interactions between particles in a fluid are extremely difficult to describe and are thus a field of research on its own. A very crude approximation of the effect of the fluid on the particles is to assume that the particles receive instantaneous, uncorrelated, random kicks [109, 110],

$$
\boldsymbol{v}_{i}^{\prime}\left(n \tau_{D}\right)=\boldsymbol{v}_{i}\left(n \tau_{D}\right)+v_{D} \boldsymbol{\xi}_{i n},
$$

at discrete times, $t_{n}=n \tau_{D}, n \in \mathbb{N}$. The $\boldsymbol{\xi}_{\text {in }}$ are gaussian random variables with zero mean, $\left\langle\boldsymbol{\xi}_{\text {in }}\right\rangle=0$, and unit variance,

$$
\left\langle\xi_{i n}^{\alpha} \xi_{j m}^{\beta}\right\rangle_{\xi}=\delta_{i j} \delta_{n m} \delta^{\alpha \beta}
$$

and $\alpha, \beta=x, y, z$ denotes the Cartesian components. In order to maintain a steady state, the energy per time dissipated in the collisions has to be replenished through the driving. In other words, the dissipative power, $\Gamma$, has to be equal to the driving power, $P_{D}=m v_{D}^{2} / 2 \tau_{D}$.

At this point, there are two parameters, $\tau_{D}$ and $v_{D}$, to control the driving but essentially we are only interested in the driving power, $P_{D}$. If we eliminate $v_{D}$ in favor of $P_{D}$, the limit of infinite driving frequency, $\tau_{D}^{-1} \rightarrow \infty$, can be performed (see appendix $B$. The kicks are now of the form

$$
\boldsymbol{v}_{i}^{\prime}(t)=\boldsymbol{v}_{i}(t)+\sqrt{2 P_{D} / m} \boldsymbol{\xi}_{i}(t),
$$

where the random variables $\boldsymbol{\xi}_{i}(t)$ describe a random walk in momentum space with zero mean and variance,

$$
\left\langle\xi_{i}^{\alpha}(t) \xi_{j}^{\beta}\left(t^{\prime}\right)\right\rangle_{\xi}=\delta_{i j} \delta^{\alpha \beta} \delta\left(t-t^{\prime}\right)
$$

and $P_{D}$ as the single control parameter.

The center of mass momentum, $\boldsymbol{P}(t) \propto \int_{0}^{t} \sum_{i} \boldsymbol{\xi}_{i}(\tau) d \tau$, inherits the properties of a random walk. It is zero on average, $\lim _{t \rightarrow \infty} \frac{1}{t} \int_{0}^{t} \boldsymbol{P}(\tau) d \tau=0$, but the spontaneous excursions will actually grow in time $\propto \sqrt{t}$. By always kicking a pair of particles, $\{i, \pi(i)\}$, at the same time with equal strength but opposite orientation, the driving 
can be made to conserve momentum. Momentum is conserved even locally, if one requires that $r_{i, \pi(i)}=\ell$, where $\ell$ is on the order of a mean particle separation. This way of driving, which is similar to the random forces used in dissipative particle dynamics (DPD) [111], restores the validity of hydrodynamics in $d=3$ [112].

Throughout this work, I will analyze inelastic hard sphere fluids that are kept in a stationary state by volume driving. I will show in the next section below and in more detail in chapter 3 that the violation of momentum conservation by volume driving will lead to long range correlations. In the subsequent chapters 5 7, I will assume that the driving conserves momentum locally.

\subsection{Dense Fluids}

\subsubsection{Spatial Correlations}

While a gas in the dilute limit is well described by point particles, the finite size of the particles influences the properties of a fluid at higher densities. In particular, the particles can no longer be regarded as spatially uncorrelated.

Spatial correlations between the particles can be partially quantified in terms of the pair correlation function, $n^{2} g\left(\boldsymbol{r}-\boldsymbol{r}^{\prime}\right)=\sum_{i \neq j}\left\langle\delta\left(\boldsymbol{r}_{i}-\boldsymbol{r}\right) \delta\left(\boldsymbol{r}_{j}-\boldsymbol{r}^{\prime}\right)\right\rangle$, which quantifies the deviation of the two particle density from its uncorrelated value, $n^{2}$. For hard sphere fluids, the contact value, $\chi \equiv g(2 a)$, of the pair correlation function is of particular importance, as it completely determines the equation of state, $p / n T=1+4 \varphi \chi(\varphi)$, where $p$ is the pressure [69]. The most widely used approximate expression for the contact value of the pair correlation function was derived by Carnahan and Starling [113,

$$
\chi_{\mathrm{CS}}(\varphi)=\frac{1+\varphi / 2}{(1-\varphi)^{3}}
$$

Its validity is discussed, e.g., in Ref. [114]. In particular, $\chi_{\mathrm{CS}}$ shows no sign of the crystallization transition and consequently, it can only be valid sufficiently far below the freezing density, $\varphi_{f}$. The mean free path, $\ell_{0}$, is directly related to contact value $\chi$ [115],

$$
a / \ell_{0}=3 \sqrt{2} \varphi \chi,
$$

as is the collision frequency, $\omega_{0}$, in the Enskog approximation [48,

$$
\omega_{E}=12 \frac{\varphi \chi}{a} \sqrt{\frac{T}{\pi m}} .
$$

Experimentally, the structure factor, $S_{q}=1+n \mathrm{FT}[g-1](q)$, is easier to measure than the pair correlation function. Upon introducing the Fourier transformed 
densities, $\rho_{\boldsymbol{q}}=\mathrm{FT}[\rho]$, where $\rho=\rho(\boldsymbol{r})$ is the density field in real space, the structure factor can alternatively be expressed as $S_{q}=\left\langle\rho_{q} \rho_{-q}\right\rangle=\left\langle\left|\rho_{q}\right|^{2}\right\rangle[69$. A particularly useful theoretical approach to calculate the static structure factor in the fluid phase was introduced by Percus and Yevick [116]. On the one hand, it allows for deriving explicit expressions in the case of hard spheres [117-119]. On the other hand it can be made quantitatively quite accurate by introducing an effective density [120].

Van Noije et al. [74] studied static correlation functions of the hydrodynamic variables density, $n$, momentum current, $m \boldsymbol{j}=n m \boldsymbol{u}$, and of the granular temperature, $T$, in a two dimensional driven granular system. Their simulation results showed growing correlation functions for small wave numbers $q$. This had also been observed by Peng and Ohta [73] before and was recently analyzed further by Head et al. [121]. Via a granular fluctuating hydrodynamics theory, van Noije et al. were able to relate this increase on large length scales to the onset of a $q^{-2}$ divergence as $q \rightarrow 0$.

\section{New Results}

The theoretical analysis in chapter 3 closely follows van Noije et al. [74]. While I found the technical reasoning mostly correct, I will conclude that the small $q$ divergence is an immediate consequence of the violation of momentum conservation by the driving which they deemed inconsequential. This is supported by the finding that upon using a driving mechanism that conserves momentum locally, the small $q$ divergence vanishes.

Static structure factors I measured in large scale simulations of systems in three dimensions will support these considerations. So far, no specific structure factor theory for (driven) inelastic hard spheres exists. Thus, these measurements will eventually be needed as input for the mode coupling theories developed in the subsequent chapters of this thesis. Independently, I will use these measurements in combination with the hydrodynamic theory to determine the speed of sound, sound damping constant and the shear viscosity.

\subsubsection{Long-Time Tails}

Let us start by looking at the diffusion of tracer particles in a host fluid. As we are not interested in mixtures, let's assume that the tracer particles are physically identical to the host particles; they are only labeled differently. The concentration, $c$, of these particles will obey a continuity equation, $\partial_{t} c=-\nabla \boldsymbol{j}$, where $\boldsymbol{j}$ is the particle current. Within the context of linear response theory, it is natural to assume that this current will be linearly related to the concentration gradient, $\boldsymbol{j}=-D \nabla c$. The constant of proportionality, $D$, is called the coefficient of diffusion or the diffusivity. From that we get the diffusion equation, $\partial_{t} c=D \Delta c$. Green [122] and Kubo [123] found that transport coefficients such as the coefficient of diffusion are related to 
the correlation functions of the corresponding or conjugate fluxes, $D=\int_{0}^{\infty} \psi(t) d t$, where, $\psi(t)=\langle\boldsymbol{v}(0) \boldsymbol{v}(t)\rangle$, is the velocity autocorrelation function (VACF). This is one manifestation of a fluctuation dissipation relation which expresses a transport coefficient in terms of the correlation function of spontaneous fluctuations.

If one simply assumes that the velocity of a tagged particle is disturbed by collisions with its surrounding particles, one would assume that the VACF is of the form, $\psi(t)=\exp \left(-t / \tau_{c}\right)$, where $\tau_{c}$ is the time scale of collisions. This implies $D \propto \tau_{c}^{-1}$. As mentioned in the introduction, an algebraic, $t^{-d / 2}$, rather than an exponential decay is found in reality. While a precise derivation of this relation needs considerable technical machinery, one can give a simple quantitative argument 69. The more rigorous theories will be discussed in chapter 5 when I will investigate the VACF for a dense granular fluid.

Imagine that because of the repeated collisions of the tagged particle with the surrounding particles of the liquid its momentum, $\boldsymbol{p}_{s}$, gets redistributed among those particles. For simplicity let us assume that the momentum gets uniformly distributed within a sphere of volume $V(t) \propto R(t)^{d}$. Because momentum transfer occurs diffusively, the size of this sphere grows as $R(t) \propto \sqrt{t}$ and thus the momentum of the tagged particle at time $t$ will be $\boldsymbol{p}_{s}(t) \propto t^{-d / 2}$ with the same result for the $\mathrm{VACF}, \psi(t) \propto\left\langle\boldsymbol{p}_{s}(0) \boldsymbol{p}_{s}(t)\right\rangle \propto t^{-d / 2}$.

The fact that the Kubo integral, $\int_{0}^{\infty} \psi(\tau) d \tau$, does not exist in two dimensions, implies that the linear diffusion law shown above does not hold in planar fluids [64]. Fortunately, it does hold in three dimensions but here the correlations impede a virial expansion of the coefficient of diffusion [65]. The latter becomes a non-analytic function of the density,

$$
D(\varphi) / D_{0}=1+D_{1} \varphi+D_{2}^{\prime} \varphi^{2} \ln \varphi+D_{2} \varphi^{2}+\ldots,
$$

including logarithmic terms [124]. For hard spheres, the low density limit is given by the Enskog diffusivity,

$$
D_{0}=\frac{a}{8 \varphi \chi} \sqrt{\pi T / m}
$$

\section{New Results}

In order to explain the simulation results on the long time tails and back scattering by Fiege et al. 67]. (see Fig. 1.4), I will derive a mode coupling theory for the tagged particle velocity in chapter 5. This allows me to describe the coupling of the tagged particle to the collective density and current modes, $\rho_{q}$, and $\boldsymbol{j}_{q}$, respectively. I will show that the coupling to the transverse current $\boldsymbol{j}_{q}^{T}$ is responsible for the long time tails, exactly like in elastic hard sphere fluid. 


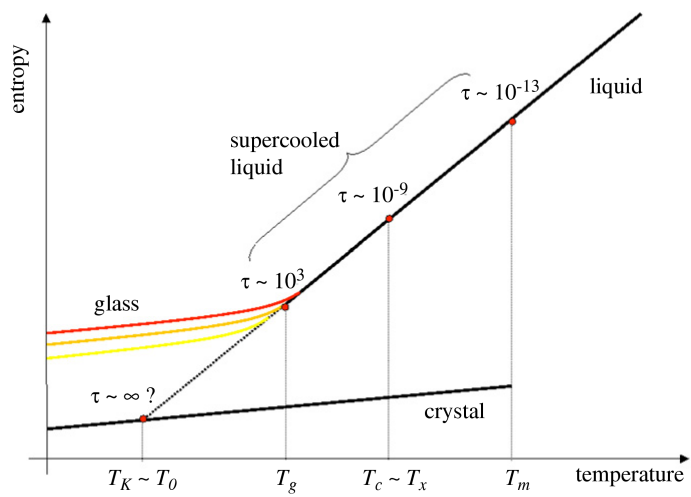

Figure 1.6: Entropy of a glass former as a function of temperature. For the relevance of the various characteristic temperatures see the main text. Reprinted from Ref. [32, Copyright (2009), with permission from Elsevier.

\subsection{Supercooled Liquids and the Glass Transition}

\subsubsection{Molecular Glass-Former}

Upon cooling, most simple fluids or melts will undergo a first oder phase transition toward a crystalline solid. In a simple fluid, the stress $\sigma$ is proportional to the strain rate, $\dot{\gamma}$, i.e, $\sigma=\eta \dot{\gamma}$. The proportionality constant, $\eta$, is defined as the fluid's viscosity. Solids, instead, build up a stress proportional to the strain, $\sigma=G \gamma$, where $G$ is the shear modulus. To describe the time dependent stress, $\sigma(t)$, in response to a varying strain rate, $\dot{\gamma}(t)$, one has to promote the shear modulus to a time dependent stress relaxation function, $G(t)$,

$$
\sigma(t)=\int_{0}^{t} d \tau G(t-\tau) \dot{\gamma}(\tau)
$$

The relation for a solid is recovered for an approximately constant stress relaxation function while in order to have a fluid, the stress relaxation function has to decay for long times, $\lim _{t \rightarrow \infty} G(t)=0$, such that $\eta=\int_{0}^{\infty} G(\tau) d \tau$ exists [125]. A A simple phenomenological expression for the shear modulus is the Maxwell model, $G(t)=$ $G_{\infty} \exp (-t / \tau)$, parametrized by the relaxation time $\tau$ [126]. This then yields the famous expression, $\eta=G_{\infty} \tau$, which states that the viscosity is directly proportional to the microscopic relaxation time. It allows for using the relaxation time and the viscosity interchangeably which is thoroughly exploited in the literature.

By following a carefully chosen protocol, it is possible to cool a lot of substances below their melting temperature without any crystallization. This can only work if it is possible, both in principle and by suitably controlling the process in an experiment, to (i) cool slower than the microscopic relaxation time, $\tau$, of the fluid in order to stay in equilibrium and (ii) to cool faster than the nucleation rate, $\tau_{N}$, for

\footnotetext{
${ }^{4}$ Note that especially if $G(t) \propto t^{-\alpha}$ for $\alpha \leq 1, G(t \rightarrow \infty)=0$ but the viscosity is still divergent. In that case the material is neither a solid nor a true fluid.
} 
the formation of crystal nuclei [127]. As both time scales depend on the temperature, $T$, it is conceivable that they eventually become equal, $\tau\left(T_{k s}\right)=\tau_{N}\left(T_{k s}\right)$. In that case it would be impossible to supercool a liquid below its kinetic spinodal, $T_{k s}$ [128]. Whether such a kinetic spinodal exists and how it could be determined is still a subject of debate [129].

As the physics of the glass transition is still not completely understood, there are a number of definitions of the concept glass transition. Not all of them are even mutually compatible. A review of all or even the most successful theories that have been proposed to (partially) explain the transition from a supercooled liquid to a glass lies beyond the scope of this work. More information can be found, e.g, in the reviews by Jäckle [130, Angell et al. [131], Ediger et al. [132], and Debenedetti and Stillinger [133] and especially the very readable introduction by Cavagna [32]. Nevertheless, I will give a brief overview of the most prominent proposals of what may define a glass transition and of the corresponding experimental signatures. This will allow me to relate the definition adopted in this thesis to the most prominent alternative definitions discussed in the literature.

I have already introduced the reaching of some arbitrary, high value of the viscosity as one indication of the glass transition. This is useful because it turns out that the value of the laboratory glass transition temperature, $T_{g}$, is not particularly sensitive to the precise value of the threshold viscosity. Upon changing the viscosity by several orders of magnitude, $T_{g}$ will only change by a few percent [134, 135].

Around $T_{g}$, the specific heat is found to drop significantly. This is attributed to the fact that at this point, the cooling rate of the experiment actually becomes faster than the relaxation rate of the system and one generates a nonequilibrium and, in particular, nonergodic state where the number of microstates accessible to the system is significantly reduced [136]. Note that simulations necessarily employ tremendous cooling rates and consequently fall out of equilibrium at comparably high temperatures [137. Up to this point, it is not obvious that the experimental glass transition at $T_{g}$ is the manifestation of a physical transition, independent of an experimental protocol.

The strong increase of the viscosity, on the other hand, hints at an actual divergence of the viscosity and indeed most of the experimental data can be fitted by the heuristic Vogel-Fulcher-Tammann"5 (VFT) law [138,140],

$$
\eta(T) \propto \exp \left(\frac{A}{T-T_{0}}\right),
$$

where $A$ and $T_{0}$ are fit parameters [141.

If $T_{0} \approx 0$, which is the case for so called strong glass formers [136], this degenerates to the Arrhenius law of activated dynamics, $\eta(T) \propto \exp (\Delta E / T)$, [142] indicating

\footnotetext{
${ }^{5}$ Note that there is a fourth author, W. Hesse
} 

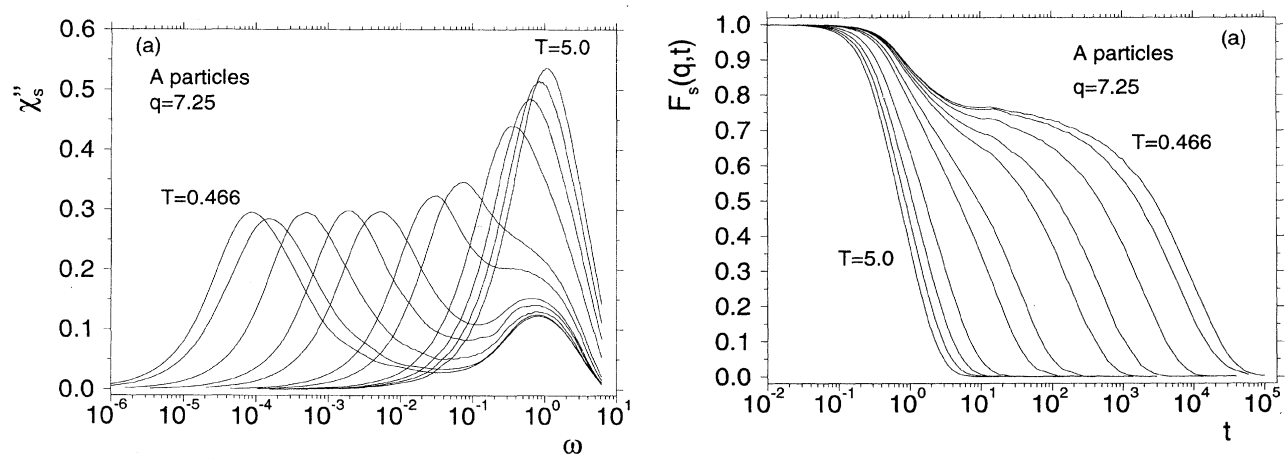

Figure 1.7: Left: Susceptibility spectrum, $\chi_{s}(\omega)$ as a function of frequency $\omega$ of a Lenard Jones fluid close to the critical temperature $T_{c}$. Right: The corresponding intermediate scattering function, $F_{s}(q, t)$, as a function of time $t$. Reprinted figures with permission from W. Kob and H. C. Andersen, Phys. Rev. E 52, 4134 (1995). Copyright (1995) by the American Physical Society.

that the system has to cross energy barriers of a characteristic, fixed height $\Delta E$. This does not provide any evidence for a (glass) transition taking place in the supercooled liquid. If $T_{0}$ significantly differs from $0 \mathrm{~K}$, the substance is said to be a fragile glass former and the dynamics must be more complicated than simple activation. In particular, there could be a transition to a new (glassy) phase close to $T_{0}<T_{g}$. More fragile glass formers will show a stronger curvature in the Angell plot, Fig. 1.2 .

From the measurement of the heat capacity, one can get the entropy of the supercooled liquid as a function of temperature, $S_{\mathrm{sc}}(T)$. Extrapolating $S_{\mathrm{sc}}$ to lower and lower temperatures, it appears to reach zero at finite temperature. As this would be unphysical, it has to change its slope at some point along that way. One candidate temperature is the so called Kauzmann temperature, $T_{K}$, where the extrapolated entropy of the supercooled liquid would cross the entropy of the crystal [128]. The Kauzmann temperature is found to be comparable to the temperature $T_{0}$ from the VFT formula above [143], lending support to the possibility of a thermodynamic glass transition.

The spectra of dynamic susceptibilities, $\chi(\omega)$, of supercooled, fragile liquids show a so called $\alpha$-peak at frequencies that are orders of magnitude below the microscopic relaxation rates [144] (see Fig. 1.7). Via the fluctuation dissipation relation, this peak in the susceptibility translates into a plateau in the corresponding dynamic correlation function, $\phi(t)$, (see Fig. 1.7). Upon lowering the temperature, the $\alpha$-peak moves to lower and lower frequencies and the plateau gets longer and longer [144]. It is natural to assume that eventually the correlations will fail to relax completely and the dynamic scattering function will instead attain a finite asymptotic value $\phi(t \rightarrow \infty)=: f_{q} \neq 0$. This would signal an intrinsic ergodic to nonergodic (ENE) or 
localization transition at a temperature $T_{c}$ which could be identified with a kinetic glass transition. Such a transition is actually found for spin glasses [145] where the nonergodicity parameter, $f_{q}$, corresponds to the Edwards-Anderson (EA) order parameter [146].

From the point of view of scattering theory, $f_{q}$ is understood as a Debye-Waller factor. Such a factor was introduced to account for the effect of thermal vibrations of atoms in a crystal lattice on scattering experiments [147, 148]. Later it was generalized to glassy, i.e. amorphous, solids [149, 150].

The long time decay from the plateau can be empirically fitted with a stretched exponential or Kohlrausch-Williams-Watts (KWW) law [151,

$$
\phi(t) \propto \exp \left[-(t / \tau)^{b}\right],
$$

where the exponent $b<1$ depends on temperature and $\tau$ is the $\alpha$-relaxation time scale. Upon expanding the $\mathrm{KWW}$ law to lowest order, one arrives at the von Schweidler law [152, 153],

$$
\phi(t)-f \propto-t^{b} .
$$

The EA order parameter, $f_{q}$, as well as the stretching exponent, $b$, were found to be independent of the details of the microscopic dynamics. In particular it is believed that the long time behavior of the dynamic correlation function, $\phi(t)$, can be matched by rescaling the time argument. This was investigated by Gleim et al. [154] for Lenard-Jones particles and for almost hard spheres by Voigtmann et al. [155]. See Ref. [156] for potential problems with this universality.

The observation that the mean square displacement, $\left\langle\delta r^{2}\right\rangle(t)=\left\langle[\boldsymbol{r}(t)-\boldsymbol{r}(0)]^{2}\right\rangle$, develops a plateau on microscopic length scales around the same temperature as the scattering function, $\phi(t)$, (see Fig. 1.8 and, e.g., Ref. [144]) inspired the so called cage picture. One imagines that upon lowering the temperature, or, what is probably more intuitive, upon increasing the density, the tagged particle gets stuck in a cage formed by the neighboring particles. It has to wiggle around in this cage for a long time, forming the plateau in $\left\langle\delta r^{2}\right\rangle(t)$, until it eventually finds an exit. This then restores the diffusive behavior, albeit with a drastically reduced diffusivity corresponding to the particle hopping from cage to cage. At $T_{c}$ the particle would be stuck in its cage forever.

A more detailed analysis of this idea goes back to Goldstein [157]. He considers the potential energy landscape of a system of particles. In the deeply supercooled regime, local clusters of particles will be stuck in minima of the potential energy with barriers which are typically of the order of several $k_{B} T$. The low probability of the activated transitions will then be responsible for the high viscosity of the supercooled liquid. This description can only be valid up to a temperature, $T_{x}$, where the depth of the local minima falls below $k_{B} T_{x}$. While the dynamics within a minimum can be 

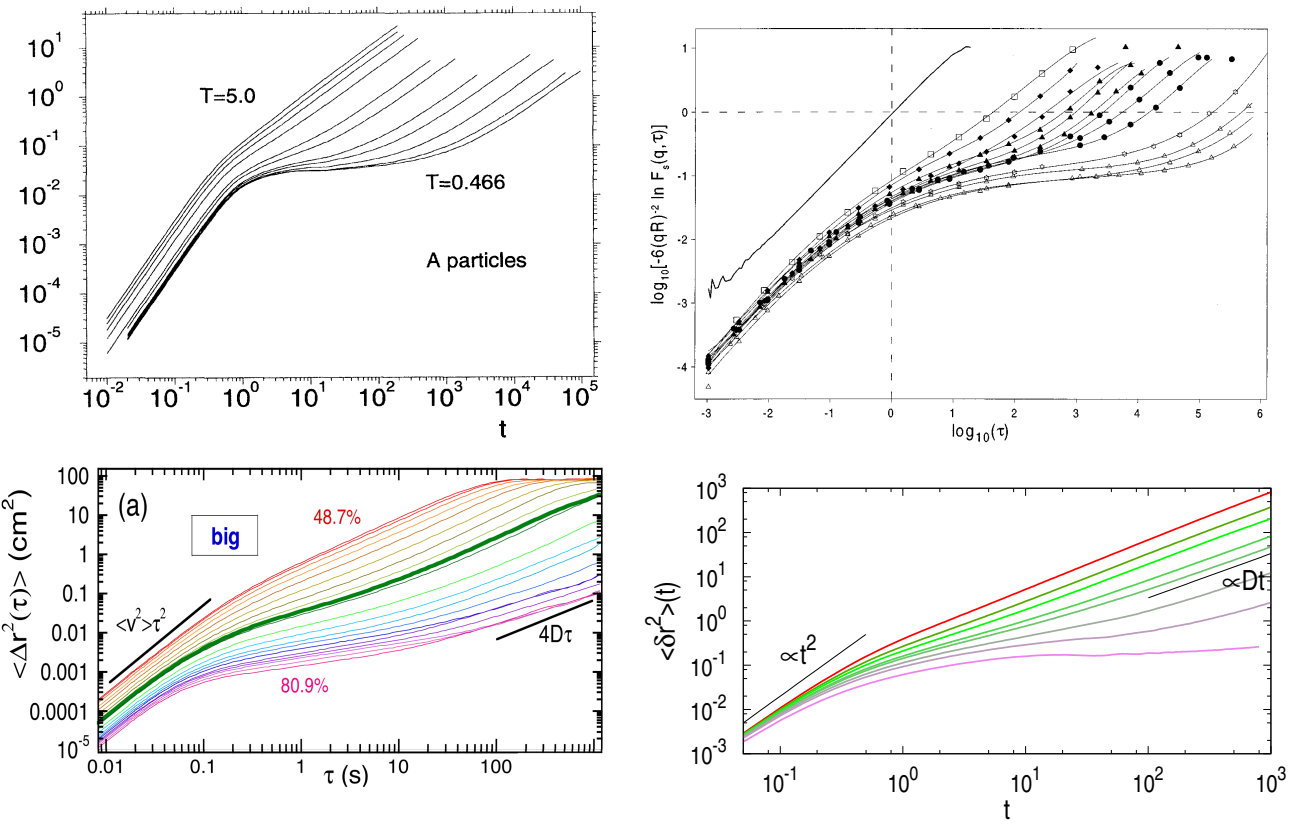

Figure 1.8: Mean square displacement as a function of time. Top Left: A Lenard Jones liquid close to the critical temperature $T_{c}$. Reprinted figure with permission from W. Kob and H. C. Andersen, Phys. Rev. E 51, 4626 (1995). Copyright (1995) by the American Physical Society. Top Right: A colloidal suspension for volume fractions $\varphi=0.466-0.583$. Reprinted figure with permission from W. van Megen, T. C. Mortensen, S. R. Williams, and J. Müller, Phys. Rev. E 58, 6073 (1998). Copyright (1998) by the American Physical Society. Bottom Left: An air fluidized granular fluid in two dimensions. Area fractions as indicated. Reprinted figure with permission from A. R. Abate and D. J. Durian, Phys. Rev. E 74, 031308 (2006). Copyright (2006) by the American Physical Society. Bottom Right: Randomly driven inelastic hard disks. Coefficient of restitution $\epsilon=0.9$ and area fractions $\varphi=0.5-0.8$. Data courtesy I. Gholami 
related to the fast $\beta$-relaxation, the slow $\alpha$-relaxation will correspond to the evolution between minima.

The idea of a kinetic glass transition, indicated by changes in the dynamics of the fluid rather than a thermodynamic glass transition indicated by nonanalyticities in the free energy was taken up by the mode coupling theory of the glass transition (MCT) (see section 4.3). MCT predicts a divergence of the $\alpha$-relaxation time at a temperature $T_{c}>T_{g}$. As MCT is formulated as a fluid dynamical theory, it does not take into account activated transport and it seems plausible that the critical temperature of $\mathrm{MCT}, T_{c}$, is related to the temperature $T_{x}$ at which activated transport becomes important [158, 159].

The types of potential glass transitions introduced above, i.e., kinetic, experimental and thermodynamical, are summarized in Fig. 1.6 together with the associated typical relaxation times $\tau$.

\subsubsection{The Colloidal Glass Transition}

Varying the concentration of colloidal suspensions, van Megen et al. [75, 160, 161] found clear indications of a kinetic glass transition (see Fig. 1.8). Only now the transition does not happen at a specific temperature, $T_{g}$, but rather at a specific density, $\varphi_{g} \approx 0.578$. At the same time, the existence of a supersaturated metastable fluid state for densities between $\varphi_{f}$ and $\varphi_{g}$ was established $\sqrt{6}$ An overview of their results can be found in Ref. [164.

While the colloidal particles in an experiment are naturally polydisperse, the first simulations of hard spheres used spheres of identical sizes for simplicity [165, 166]. More recent studies with larger system sizes then found that it is hardly possible to supersaturate monodisperse hard sphere fluids significantly [167] and that even samples that are initially perfectly amorphous, quickly crystallize [168, 169]. To avoid the crystallization, polydisperse mixtures are used to stabilize the glassy phase. Already binary mixtures where the big particles are about $10 \%$ larger than the small particles were found to slow down crystal nucleation sufficiently [170, 168] to allow for substantial supersaturation. Recently, the role of fractionation ${ }^{7}$ has been emphasized [171-173].

Simulations of hard sphere fluids at high densities were performed both with Newtonian and with Brownian dynamics. A kinetic glass transition could be located at $\varphi_{c} \approx 0.58$ which, surprisingly, coincides with the experimental value, $\varphi_{g}$. It has been argued that activated transport, which allows the material to remain fluid for temperatures $T<T_{x}$, is suppressed in colloidal suspensions [174] and that therefore the localization transition at $\varphi_{c}$ is actually the relevant glass transition.

\footnotetext{
${ }^{6}$ Although in most experiments the density and not the pressure is used as a control variable, the terms supercompressed or even supercooled are used most of the time. Notable exceptions are Refs. 162, 163 .

${ }^{7}$ I.e., the demixing of the species
} 
Experiments and simulations indicate that a colloidal glass can be molten by applying a shear that exceeds a specific yield stress [175-177]. Fuchs and Cates have extended MCT to describe this behavior [178 180]. In these experiments the energy input of the shearing force is dissipated through the viscosity of the host fluid of the colloidal suspension. The existence of a finite yield stress and a related glass transition driven by an external shear fits well into the concept of the jamming diagram (Fig. 1.3).

\subsubsection{The Granular Glass Transition}

In measurements of the mean square displacement, $\left\langle\delta r^{2}\right\rangle(t)$, in quasi two dimensional systems of air fluidized [42] or vertically vibrated [44] steel beads, a plateau reminiscent of the one found close to the localization transition was observed (see Fig. 1.8). While the influence of wall induced ordering is not easy to rule out, these experiments hint at a granular kinetic glass transition.

Simulations of the randomly driven granular fluid also display this behavior (see Fig. 1.8). Different from the experiments, the coefficient of restitution can easily be varied in a simulation. Measurements of the mean square displacement at a constant volume fraction close to the critical value $\varphi_{c}$ for a fluid of elastic hard spheres show that upon decreasing the coefficient of restitution, the plateau becomes shorter or even vanishes [181].

\section{New Results}

In chapter 6 I will extend mode coupling theory to the nonequilibrium stationary state of a randomly driven granular fluid. The resulting theory predicts a kinetic glass transition for all values of the coefficient of restitution $\epsilon$ (Fig. 1.9p). The critical density $\varphi_{c}(\epsilon)$, increases continuously from the elastic value, i.e., a kinetic glass transition occurs also in a driven granular fluid but at a higher density compared to a colloidal suspension. Close to the critical density, $\varphi_{c}(\epsilon)$, the familiar formation of a plateau is found in the dynamic scattering function, $\phi(q, t)$ (Fig. 1.9).

A second type of mode coupling theory (see chapter 7), allows me to derive predictions for the incoherent scattering function and, even more important, the mean square displacement, $\left\langle\delta r^{2}\right\rangle(t)$, of a driven granular fluid. The predictions are in qualitative agreement with the results from physical and numerical experiments (Fig. 1.8) that raised the question of an granular glass transition.

Both the critical EA order parameter ${ }^{8} f_{q}^{c}=f_{q}^{c}(\epsilon)$, and the exponent $b=b(\epsilon)$ in the von Schweidler law (1.17) are found to depend on the coefficient of restitution, $\epsilon$. This

\footnotetext{
${ }^{8}$ I choose the term Edwards-Anderson order parameter to emphasize that I use it to identify the kinetic glass transition. As little is known about the ergodicity of driven granular fluids and scattering experiments of granular assemblies are still being developed, I avoid the alternative terms nonergodicity parameter and Debye-Waller factor.
} 

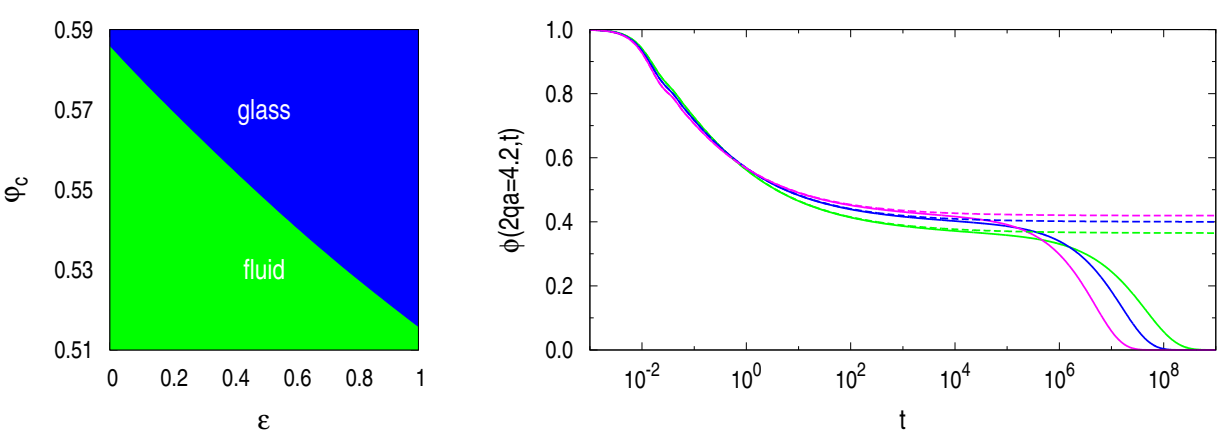

Figure 1.9: Results of the granular MCT. Left: The critical density, $\varphi_{c}$, of the granular kinetic glass transition as a function of the coefficient of restitution, $\epsilon$. Right: The coherent scattering function, $\phi(q, t)$, as a function of time $t$ at wave number $2 q a=4.2$, for a coefficient of restitution $\epsilon=1.0$ (green), 0.5 (blue), 0.0 (magenta). The dashed lines correspond to a granular glass at the crictial volume fraction, $\varphi_{c}(\epsilon)$, while the solid lines are for a granular fluid at a reduced density $\Delta \varphi(\epsilon) \equiv\left[\varphi_{c}(\epsilon)-\varphi\right] / \varphi_{c}(\epsilon)=10^{-3}$, close to the critical density.

indicates that the granular dynamics close to the glass transition is fundamentally different from either the Newtonian dynamics of hard spheres or the Brownian dynamics of colloidal suspensions. The dissipative, granular dynamics can not be mapped to the equilibrium dynamics be rescaling the unit of time. The same holds for the mean square displacement, $\left\langle\delta r^{2}\right\rangle(t)$, and the incoherent scattering function, $\phi^{s}(q, t)$, where the localization length, $r_{c}=r_{c}(\epsilon)$, and the plateau height, $f_{q}^{s c}=f_{q}^{s c}(\epsilon)$, also depend on the coefficient of restitution.

\subsection{Mode Coupling Theories}

The central results of this thesis (see chapters 5 77) have been obtained by extending mode coupling theory to the nonequilibrium steady state of a driven granular fluid. A brief introduction to standard mode coupling theory and its extensions therefore seems in order.

Fixman [182] was supposedly the first to have used mode coupling ideas. He wanted to calculate the viscosity of a liquid close to its critical point via the corresponding Green-Kubo expression. For this he needed precise expressions for the stress autocorrelation function. Fixman's ideas were generalized and formalized by Kadanoff and Swift [183] and Kawasaki [184, 185] using projection operators. With the advent of renormalization group techniques, the use of mode coupling theory to calculate transport coefficients close to the critical point was soon superseded (See Ref. [186] for a review that relates the two approaches.) Mode coupling continued 
to be successful, though, for the calculation of dynamic correlation functions and consequently, a number of groups developed mode coupling equations employing various approaches. Rather comprehensive reviews have been provided by Pomeau and Resibois [62], Oppenheim [187] and more recently Das [188]. These authors all wanted to describe simple liquids, but mode coupling theory has also been applied to magnetic phenomena [189, 190] and the direct interaction approximation [191] employed in the field of turbulence appears to be a closely related approach.

There are in fact several theories that go by the name mode coupling theory 9 I will show that at least three of those can be extended to the nonequilibrium stationary state of a driven granular fluid. As any discussion of mode coupling theory is necessarily rather technical, here I can give only a glimpse of the defining aspect, i.e., a certain type of approximation for the memory kernel. A more technical discussion will follow in chapter 4

I am going to discuss a number of dynamic correlation functions, $\phi_{a}(t)$, including the VACF, $\psi(t)$, the coherent scattering function, $\phi(q, t)$, and its incoherent counterpart, $\phi^{s}(q, t)$. All of these are solutions of either of two types of equations of motion [115,

$$
\begin{gathered}
\dot{\phi}_{a}(t)+\nu \phi_{a}(t)+\int_{0}^{t} M_{a}(t-\tau) \phi_{a}(\tau) d \tau=0, \\
\ddot{\phi}_{a}(t)+\nu \dot{\phi}_{a}(t)+\Omega^{2} \phi_{a}(t)+\int_{0}^{t} M_{a}(t-\tau) \dot{\phi}_{a}(\tau) d \tau=0,
\end{gathered}
$$

where $\nu$ and $\Omega$ are characteristic relaxation rates and the function $M_{a}(t)$ is called the memory kernel. While the relaxation rates $\nu$ and $\Omega$ can in principle be determined exactly, apart from special cases one has to resort to approximations for the memory kernel $M_{a}(t)$. One of the many approximation schemes that have been used (see, e.g., Ref. [115]), is the mode coupling approximation,

$$
M_{a}(t) \approx M_{a}^{\mathrm{MCT}}(t)=\sum_{b, c} \mathcal{V}_{a b c}^{2} \phi_{b}(t) \phi_{c}(t),
$$

which assumes that the time dependence of the memory kernel's dominant part can be written as a product of two dynamic correlation functions, $\phi_{b}(t)$ and $\phi_{c}(t)$. The so called vertices, $\mathcal{V}_{a b c}$, are time independent.

In chapter $4 \mathrm{I}$ will try to further motivate the form of the equations of motion and the chosen mode coupling approximations for the correlation functions under consideration; Namely the VACF $\psi(t)$ (chapter 5), the coherent scattering function $\phi(q, t)$ (chapter 6) and the incoherent scattering function $\phi^{s}(q, t)$ (chapter 7 ).

\footnotetext{
${ }^{9}$ The older literature also sometimes uses the term mode-mode coupling theory.
} 



\section{Microscopic Dynamics and Averages}

The microscopic dynamics of particles whose interactions can be described by an interaction potential $V$, is fully described by the Hamiltonian equations of motion. A particularly powerful reformulation of these equations of motion employs the Liouville operator, $\mathcal{L}_{+}$, which I will introduce in the following section. Already the hard sphere interaction introduces some subtleties into this description that I will indicate in the subsequent section 2.2. For the driven inelastic hard sphere system, I will give a description of the driving in terms of a suitable Liouville operator. In the nonequilibrium stationary state of the driven granular fluid, the probability distribution of the microscopic degrees of freedom is no longer given by the canonical distribution function. While there are some results on the form of this nonequilibrium distribution function, I still need to specify an approximate form that I will use in the following chapters. Lastly, I will relate the violation of detailed balance in the nonequilibrium stationary state to the fact that the adjoint Liouville operator, $\mathcal{L}_{+}^{\dagger}$, is no longer given by the time reversed operator, $\mathcal{L}_{-}$.

\subsection{Conservative Dynamics}

As is known from every text book on classical mechanics (e.g., Ref. [192]), the dynamics of particles interacting by conservative forces, can be determined as the solution of the Hamiltonian equations of motion. I will first introduce the concepts for such a Hamiltonian system as the Hamiltonian requirements avoid most of the subtleties introduced when I will generalize these concepts to hard spheres and eventually inelastic hard spheres that are both necessarily nonconservative [193].

In all of these cases the state of an $N$ particle system is described uniquely by specifying the positions $\boldsymbol{q} \equiv\left(\boldsymbol{r}_{1}, \ldots, \boldsymbol{r}_{N}\right)$ and momenta $\boldsymbol{p} \equiv\left(\boldsymbol{p}_{1}, \ldots, \boldsymbol{p}_{N}\right)$ of all the particles. The pair, $(\boldsymbol{q}, \boldsymbol{p}) \in \Gamma$, specifies the location of the system in the corresponding phase space $\Gamma$.

Within the Hamiltonian formulation of classical mechanics, the dynamics of the positions and momenta follows from the Hamiltonian equations of motion,

$$
\dot{\boldsymbol{q}}=\frac{\partial H}{\partial \boldsymbol{p}}, \quad \dot{\boldsymbol{p}}=-\frac{\partial H}{\partial \boldsymbol{q}},
$$

where $H=\boldsymbol{p}^{2} / 2 m+V(\boldsymbol{q})$ is the Hamiltonian corresponding to an interaction potential $V(\boldsymbol{q})=\sum_{i<j} V\left(\left|\boldsymbol{r}_{i}-\boldsymbol{r}_{j}\right|\right)$. 
On the microscopic level, every observable $A$ must be a function of the phase space coordinate $(\boldsymbol{q}, \boldsymbol{p})$ and possibly of the time, $A=A(\boldsymbol{q}, \boldsymbol{p}, t)$. The time evolution of the observable $A$ can be expressed with the help of the Poisson brackets $\{\cdot, \cdot\}$ as

$$
\frac{d A}{d t}=\{H, A\}+\frac{\partial A}{\partial t}=: i \mathcal{L} A+\frac{\partial A}{\partial t},
$$

defining the Liouville operator $\mathcal{L}$ [194]. The explicit imaginary unit in this definition is purely conventional and was introduced to make formal contact with the time evolution operator used in quantum mechanics. For the simple generic system specified above the Liouville operator reads,

$$
i \mathcal{L}=i \mathcal{L}_{0}+\sum_{i<j} \mathcal{L}_{i j}^{I}=\frac{\boldsymbol{p}}{m} \cdot \frac{\partial}{\partial \boldsymbol{q}}+\sum_{i<j} \frac{\partial V\left(r_{i j}\right)}{\partial \boldsymbol{r}_{i j}} \cdot\left(\frac{\partial}{\partial \boldsymbol{p}_{i}}-\frac{\partial}{\partial \boldsymbol{p}_{j}}\right) .
$$

For an observable that does not depend on time explicitly, the time evolution equation 2.2 can be solved formally to yield an expression for the propagator, $U\left(t, t^{\prime}\right)$,

$$
A(t)=U(t, 0) A(0)=\exp (i t \mathcal{L}) A(0),
$$

where the exponential operator, exp, is understood as a shorthand, $\exp \mathcal{O}:=$ $\sum_{n} \mathcal{O}^{n} / n$ !, assuming that this power series converges.

The ensemble average, $\langle A ; t\rangle$, at time $t$ of an observable $A$ can be defined as an average over the distribution of initial conditions, $\varrho(\Gamma, t=0)$,

$$
\langle A ; t\rangle=\int d \Gamma \varrho(\Gamma, 0) \exp (i t \mathcal{L}) A(\Gamma)=\int d \Gamma \varrho(\Gamma, 0) A(\Gamma ; t) .
$$

Unless it can be the source of confusion, I will usually drop the time argument and use $\langle A\rangle \equiv\langle A ; t\rangle$. To introduce yet more notation, one can regard this average as a scalar product, $\langle A ; t\rangle=(\varrho, A(t))$. From $(\varrho, A(t))=(\varrho, \exp (i t \mathcal{L}) A)$ one then immediately finds

$$
\frac{d}{d t}\langle A ; t\rangle=(\varrho, \exp (i t \mathcal{L}) i \mathcal{L} A)=(\varrho, \dot{A}(t))=\langle\dot{A} ; t\rangle,
$$

i.e., time derivatives and the averaging commute.

The scalar product notation suggests the introduction of an adjoint operator,

$$
(\varrho, i \mathcal{L} A)=:(i \overline{\mathcal{L}} \varrho, A) .
$$

It is possible to [195], that this operator can be used to transfer the average from the Heisenberg representation used above to the Schrödinger representation,

$$
\langle A ; t\rangle=\int d \Gamma \varrho(\Gamma, t) A(\Gamma)=\int d \Gamma[\exp (i t \overline{\mathcal{L}}) \varrho(\Gamma, 0)] A(\Gamma),
$$


where the integration now no longer runs over the initial conditions but over the phase space, weighted with its density at time $t, \varrho(\Gamma, t)$. It is mostly this representation that I will use in the rest of this work.

The fact that the time evolution of the distribution function is given by $i \overline{\mathcal{L}}$, can also be expressed as the Liouville equation,

$$
\frac{\partial \varrho}{\partial t}+i \overline{\mathcal{L}} \varrho=0 .
$$

For Hamiltonian systems, the Liouville operator is self-adjoint, $\overline{\mathcal{L}}=\mathcal{L}$, and the Liouville equation also holds with the Liouville operator, $\mathcal{L}$, itself [196].

Although the distribution function, $\varrho$, is well defined as the solution of the Liouville equation 2.9 this is, apart from simple examples, only a formal specification. For most systems the Liouville equation can not be solved explicitly. A very usefull set of identities to derive approximations is the so called BBGKY-hierarchy [69

To make contact with notation used in the field, let $f_{N}=N \varrho$ and let $f_{n}\left(x_{1}, \ldots, x_{n}\right)=$ $\frac{N !}{(N-n) !} \int d x_{n+1} \cdots d x_{N} f_{N}\left(x_{1}, \ldots, x_{N}\right)$ be the distribution function of the first $n$ particles. Then one can derive the following exact identity,

$$
\left(\frac{\partial}{\partial t}+\sum_{i=1}^{n} \frac{\boldsymbol{p}_{i}}{m} \nabla_{i}\right) f_{n}+\sum_{i=1}^{n} \int d x_{n+1} i \mathcal{L}_{i, n+1}^{I} f_{n+1}=0,
$$

which relates the $n$-particle distribution function, $f_{n}$, to the $(n+1)$-particle distribution function, $f_{n+1}$ [69]. As it stands, the BBGKY-hierarchy does not help a lot as it expresses, e.g., the unknown single particle distribution, $f_{1}$, in terms of the two particle distribution function, $f_{2}$, that usually is even less known. On the other hand, one can argue that the single particle distribution function will depend less and less sensitvely on the higher order distribution function in this hierarchy. Thus, one might be able to truncate the hierarchy by applying some approximation to one of these higher oder functions. In fact, the famous Boltzmann equation can be understood as the realisation of such a procedure.

From the first two equations of this hierarchy and the assumption that (i) the duration of a collision is much shorter than the mean time between collisions (which will be fulfilled in the limit of low density and short ranged interaction potentials) and (ii) the two-particle distribution function, $f_{2}$, factorizes with respect to the velocities, i.e., $f_{2}\left(\boldsymbol{r}, \boldsymbol{v}_{1}, \boldsymbol{r}, \boldsymbol{v}_{2}\right)=f_{1}\left(\boldsymbol{r}, \boldsymbol{v}_{1}\right) f_{1}\left(\boldsymbol{r}, \boldsymbol{v}_{2}\right)$ (called molecular chaos) one can find a closed equation for the one-particle distribution function,

$$
\left(\frac{\partial}{\partial t}+\boldsymbol{v}_{1} \nabla\right) f_{1}=J\left[f_{1}\right]
$$

the Boltzmann equation, where,

$$
J\left[f_{1}\right]=\int d^{d} v_{2} \int d \Omega \frac{\partial \sigma}{\partial \Omega} v_{12}\left[f_{1}\left(\boldsymbol{v}_{1}^{\prime}\right) f_{1}\left(\boldsymbol{v}_{2}^{\prime}\right)-f_{1}\left(\boldsymbol{v}_{1}\right) f_{1}\left(\boldsymbol{v}_{2}\right)\right],
$$


is the collision integral and the primed quantities are the values of the velocities after a collision. The term $\partial \sigma / \partial \Omega$ denotes the scattering cross section, $\sigma$, per solid angle, $\Omega$ [69]. The Boltzmann equation is one possible starting point to derive the hydrodynamic equations [48].

A special case of a relation from the BBGKY hierarchy is the Yvon-Born-Green (YBG) theorem for the pair correlation function, $g(r)$ [197]. From the definition,

$$
g\left(r_{12}\right)=V^{2} \int d^{3} r_{3} \cdots d^{3} r_{N} \prod_{i<j} \varrho_{r 1}\left(r_{i j}\right)
$$

where $\varrho_{r 2}\left(r_{i j}\right) \propto \exp \left[-\beta V\left(r_{i j}\right)\right]$ is the spatial distribution function one finds,

$$
\begin{aligned}
\nabla_{1} g\left(r_{12}\right) & =V^{2} \int d^{3} r_{3} \cdots d^{3} r_{N}\left[\nabla_{1} \ln \varrho_{r 2}\left(r_{12}\right)+\sum_{i} \nabla_{1} \ln \varrho_{r 2}\left(r_{1 i}\right)\right] \varrho_{N} \\
& =g\left(r_{12}\right) \nabla_{1} \ln \varrho_{r 2}\left(r_{12}\right)+n \int d^{3} r_{3} g_{3}\left(\boldsymbol{r}_{1}, \boldsymbol{r}_{2}, \boldsymbol{r}_{3}\right) \nabla_{1} \ln \varrho_{r 2}\left(r_{13}\right)
\end{aligned}
$$

which relates the pair correlation function to a particular integral of the triplet correlation function, $g_{3}\left(\boldsymbol{r}, \boldsymbol{r}_{2}, \boldsymbol{r}_{3}\right)$. With the special form of the canonical distrbution function, this can be further simplified to

$$
n \beta \int d^{3} r_{3} g_{3}\left(\boldsymbol{r}_{1}, \boldsymbol{r}_{2}, \boldsymbol{r}_{3}\right) \nabla_{1} V\left(r_{13}\right)=-\beta g\left(r_{12}\right) \nabla_{1} V\left(r_{12}\right)-\nabla_{1} g\left(r_{12}\right) .
$$

In the same way, one can also derive the higher order analogues of this relation.

In the chapters on mode coupling theory I will need to calculate matrix elements, $\langle A \mid \mathcal{L} B\rangle \equiv\left\langle A^{*} \mathcal{L} B\right\rangle$, of the Liouville operator with respect to the observables $A$ and $B$. If one considers this kind of average as a scalar product, it is natural to introduce the adjoint Liouville operator, $\mathcal{L}^{\dagger}$, defined via

$$
\left\langle\mathcal{L}^{\dagger} A \mid B\right\rangle=\langle A \mid \mathcal{L} B\rangle
$$

From the sequence of identities,

$$
\left(\varrho \mathcal{L}^{\dagger} A, B\right)=\left\langle\mathcal{L}^{\dagger} A \mid B\right\rangle=\langle A \mid \mathcal{L} B\rangle=(\varrho A, \mathcal{L} B)=(\overline{\mathcal{L}} \varrho A, B)
$$

one finds

$$
\mathcal{L}^{\dagger} A=\varrho^{-1} \overline{\mathcal{L}} \varrho A,
$$

where $\varrho^{-1}$ is the pseudo inverse of the phase space distribution function. In the Hamiltonian case, the Liouville operator is a differential operator such that this can be simplified to

$$
\mathcal{L}^{\dagger} A=\overline{\mathcal{L}} A+A \varrho^{-1} \overline{\mathcal{L}} \varrho
$$


and due to the Liouville equation $(2.9)$, the adjoint operators with respect to both scalar products are actually identical,

$$
\mathcal{L}^{\dagger}=\overline{\mathcal{L}}=\mathcal{L}
$$

Because of the first identity, the free streaming and interaction part, are also self adjoint separately, i.e., $\mathcal{L}_{0}^{\dagger}=\mathcal{L}_{0}$.

Moreover, the Hamiltonian equations of motion are invariant under time reversal, $t \rightarrow-t$, which implies that the forward in time Liouville operator, $\mathcal{L}_{+}$, is identical to the backward in time Liouville operator, $\mathcal{L}_{-}$. Detailed balance, valid in equilibrium systems, implies that $\left\langle\mathcal{L}_{-} A \mid B\right\rangle=\left\langle A \mid \mathcal{L}_{+} B\right\rangle$ (see, e.g., Ref. [198]), which in turn requires that $\mathcal{L}_{ \pm}^{\dagger}=\mathcal{L}_{\mp}$. Microscopic time reversal invariance, $\mathcal{L}_{+}=\mathcal{L}_{-}$, combined with the result that the Liouville operator is self adjoint is compatible with this requirement.

\subsection{Hard Spheres}

For hard spheres the definition of the Liouville operator via the Poisson bracket certainly is not applicable. Nevertheless, it is still a deterministic system such that the propagator $U\left(t, t^{\prime}\right)$ must exist. The free streaming, $U_{0}(t, 0)=\exp \left(i t \mathcal{L}_{0}\right)$, of the particles remains the same. It is only the hard core interactions that need to be considered carefully. The fact that the particles experience periods of free streaming interrupted by instantaneous pairwise collisions can be captured in the following recursive equation, [199]

$$
U(t, 0)=U_{0}(t, 0)+\sum_{j, k} \int_{0}^{t} d \tau U(t, \tau) i T_{j k}^{+} U_{0}(\tau, 0),
$$

where $i T_{i j}^{+}$is the (forward in time) binary collision operator detailed below. Iterating the above equation once and expanding it up to second order in time, we get,

$$
\begin{aligned}
U(t, 0)=1 & +i t \mathcal{L}_{0}(0)-\frac{1}{2} t^{2} \mathcal{L}_{0}^{2}(0) \\
& +\sum_{j<k} \int_{0}^{t} d \tau\left[1+i(t-\tau) \mathcal{L}_{0}(\tau)\right] i T_{j k}^{+}(\tau)\left[\left(1+i \tau \mathcal{L}_{0}(0)\right)\right] \\
& +\sum_{j, k, \ell, m}^{\prime} \int_{0}^{t} d \tau \int_{\tau}^{t} d \tau^{\prime} i T_{j k}^{+}\left(\tau^{\prime}\right) i T_{\ell m}^{+}(\tau)+\mathcal{O}\left(t^{3}\right)
\end{aligned}
$$

where the primed multisum runs over all pairwise distinct indices. If one insists to write the propagator $U$ in terms of a generator, the pseudo Liouville operator 
$\hat{\mathcal{L}}_{+}=\mathcal{L}_{0}+\sum_{j<k} \mathcal{T}_{j k}^{+}=\mathcal{L}_{0}+\mathcal{T}_{+}[200]$, one finds that $\hat{\mathcal{L}}_{+}^{2} \neq \hat{\mathcal{L}}_{+} \hat{\mathcal{L}}_{+}$but

$$
\hat{\mathcal{L}}_{+}^{2}:=\mathcal{L}_{0}^{2}+\sum_{j<k}\left[\mathcal{L}_{0} \mathcal{T}_{j k}^{+}+\mathcal{T}_{j k}^{+} \mathcal{L}_{0}\right]+\sum_{j, k, \ell, m}^{\prime} \mathcal{T}_{j k}^{+} \mathcal{T}_{\ell m}^{+}
$$

and similar for higher powers of $\hat{\mathcal{L}}_{+}$. A related approach led Ernst et al. [199] to introduce the binary collision operators. The power series for the exponential operator, $\exp \left(i t \hat{\mathcal{L}}_{+}\right)$, must then also be understood in terms of this modified product rule 11 For a critique of the pseudo Liouville operator and an alternative approach, see Ref. [201].

The (forward or backward in time) collision operator, $i \mathcal{T}_{j k}^{ \pm}$, can be written as

$$
i \mathcal{T}_{j k}^{ \pm} \equiv \lim _{t \rightarrow 0} \frac{1}{t} \int_{0}^{t} d \tau i T_{j k}^{ \pm}(\tau)=-\left(\hat{\boldsymbol{r}}_{j k} \cdot \boldsymbol{v}_{j k}\right) \Theta\left(\mp \hat{\boldsymbol{r}}_{j k} \cdot \boldsymbol{v}_{j k}\right) \delta\left(r_{j k}-2 a\right)\left(b_{j k}-1\right),
$$

where the operator $b_{i j}$ implements the collision rules 1.2 (with $\left.\epsilon=1\right)$ [100].

Detailed balance also holds for an hard sphere fluid in thermal equilibrium. Therefore the adjoint of the Liouville operator is simply related to the time reversed operator, $\hat{\mathcal{L}}_{ \pm}^{\dagger}=\hat{\mathcal{L}}_{\mp}$, which only differs in the sign inside the Heaviside step function, $\Theta(\cdot)$, of the collision operator. Note, that the free streaming operator, $i \mathcal{L}_{0}$, even though it is formally identical to the one for Hamiltonian systems, is no longer self adjoint. In fact one can show from eq. (2.19) that $i \mathcal{L}_{0}^{\dagger}=i \mathcal{L}_{0}+\sum_{j<k}\left(\hat{\boldsymbol{r}}_{j k} \cdot \boldsymbol{v}_{j k}\right) \delta\left(r_{j k}-2 a\right)$.

Due to the degenerate form of the hard sphere potential, the spatial distribution function, $\varrho_{r}(\boldsymbol{q})=\lim _{\beta \rightarrow \infty} \prod_{i<j} \exp \left[-\beta \Theta\left(2 a-r_{i j}\right)\right]=\Theta\left(r_{i j}-2 a\right)$, is not immediately recognizable as the usual Boltzmann factor that it is. Its interpretation is, that all configurations that are overlap free are equally probable. With $\nabla_{1} \ln \varrho_{r}\left(r_{12}\right)=$ $\Theta\left(r_{12}-2 a\right) \delta\left(r_{12}-2 a\right) \hat{\boldsymbol{r}}_{1}$, the YBG theorem for hard spheres reads

$$
n \int d^{3} r_{3} g_{3}\left(\boldsymbol{r}_{1}, \boldsymbol{r}_{2}, \boldsymbol{r}_{3}\right) \delta\left(r_{13}-2 a\right) \hat{\boldsymbol{r}}_{1}=\nabla_{1} g\left(r_{12}\right)-\chi \delta\left(r_{12}-2 a\right) \hat{\boldsymbol{r}}_{1} .
$$

\subsection{Inelastic Hard Spheres}

The dissipative interactions of inelastic hard spheres ${ }^{2}$ can also be described by a pseudo Liouville operator,

$$
\mathcal{L}_{+}=\mathcal{L}_{0}+\sum_{j<k} \mathcal{T}_{j k}^{+}+\mathcal{L}_{D}^{+}
$$

where,

$$
i \mathcal{T}_{j k}^{+}=-\left(\hat{\boldsymbol{r}}_{j k} \cdot \boldsymbol{v}_{j k}\right) \Theta\left(-\hat{\boldsymbol{r}}_{j k} \cdot \boldsymbol{v}_{j k}\right) \delta\left(r_{j k}-2 a\right)\left(b_{j k}^{+}-1\right),
$$

\footnotetext{
${ }^{1}$ Although the pseudo Liouville operator is widely and successfully used, I am not aware of a mathematically rigorous discussion of its nontrivial algebraic properties.

${ }^{2}$ It appears that this dynamics can also be interpreted in terms of an economic model 202
} 
and $b_{j k}^{+}$implements the inelastic collision rules $(1.2)$ [203, 204]. In the limit of infinite driving frequency, the Liouville operator corresponding to the driving force can be written as a differential operator (see appendix B and Ref. [205]),

$$
i \mathcal{L}_{D}^{+}=\sum_{i} \boldsymbol{\xi}_{i}(t) \cdot \frac{\partial}{\partial \boldsymbol{v}_{i}}+\frac{P_{D}}{m} \frac{\partial^{2}}{\partial \boldsymbol{v}_{i}^{2}} .
$$

There is no reason to believe that detailed balance holds in the nonequilibrium driven granular fluid. The stationary state is time translation invariant but no longer time reversal invariant. By looking at the inelastic collisions one can easily detect the direction of time. To obtain an explicit expression for the adjoint pseudo Liouville operator one has to go back to eq. (2.18). Fortunately, it turns out that knowledge of the adjoint pseudo Liouville operator, $\mathcal{L}_{+}^{\dagger}$, is not strictly needed for any of the following calculations.

No exact analytical expression analogous to the canonical distribution function of the elastic hard sphere system is known for the driven inelastic hard sphere fluid. I will follow the common assumption that the distribution function, $\varrho(\Gamma)$, can be factorized into a velocity distribution, $\varrho_{v}(\boldsymbol{p})$, and a spatial distribution, $\varrho_{r}(\boldsymbol{q})$, i.e., $\varrho(\Gamma)=\varrho_{r}(\boldsymbol{q}) \varrho_{v}(\boldsymbol{p})$.

Not much is known about the spatial part. For the homogeneous and isotropic systems considered here, it can only depend on the distances between all the particles, $\varrho_{r}=\varrho_{r}\left(\left\{r_{i j}\right\}\right)$, and overlapping configurations must have zero weight. From that I conclude that it can be written in the form,

$$
\varrho_{r}\left(\left\{r_{i j}\right\}\right) \propto \prod_{i<j} \Theta\left(r_{i j}-2 a\right) \vartheta\left(r_{i j}\right),
$$

with an unknown function $\vartheta(r)$. Often it is assumed that the expressions for the pair correlation function or the static structure factor of an elastic hard sphere system are also approximately valid for inelastic hard sphere fluids. This corresponds to the assumption $\vartheta(r) \equiv 1$. In chapter 3 below, I will show explicitly that the static structure factor of a driven granular fluid is not identical to that of the corresponding elastic hard sphere fluid at the same density. Nevertheless, I will also use the approximation $\vartheta(r)=1$ as a simplifying assumption in the subsequent chapters.

As far as the velocity distribution function is concerned, it was found that the one-particle velocity distribution function, $\varrho_{v 1}(\boldsymbol{v})$, is not a gaussian, but that the high velocity tails are overpopulated (see, e.g., Ref. [206]). For an analysis of the molecular chaos assumption in driven granular fluids, $\varrho_{v 2}\left(\boldsymbol{v}_{i}, \boldsymbol{v}_{j}\right)=\varrho_{v 1}\left(\boldsymbol{v}_{i}\right) \varrho_{v 1}\left(\boldsymbol{v}_{j}\right)$, see Ref. [207]. I will only need the first two moments of the velocity distribution, $\langle\boldsymbol{v}\rangle=\mathbf{0}$ and, $\left\langle\boldsymbol{v}^{2}\right\rangle=3 T / m<\infty$. Therefore I assume without loss of generality a gaussian form of the one-particle velocity distribution function,

$$
\varrho_{v 1}(\boldsymbol{v}) \propto \exp \left[-m \boldsymbol{v}^{2} / 2 T\right],
$$


parametrized by the granular kinetic temperature $T$. Let me stress here that the severe approximation is the factorization of the velocity distribution function. Once this is done, the quantities I am going to calculate do not depend on non-gaussian corrections of the one particle velocity distribution function.

In conclusion, I will use the following approximate stationary distribution function,

$$
\varrho(\Gamma) \propto \prod_{i<j} \Theta\left(r_{i j}-2 a\right) \vartheta\left(r_{i j}\right) e^{-m v_{i}^{2} / 2 T} .
$$

With $\vartheta\left(r_{i j}\right) \equiv 1$, this is the exact distribution function of a fluid of elastic hard spheres in thermal equilibrium.

To get results that are independent of a specific realization of the driving, I will include an average over the distribution of the random force, $P(\boldsymbol{\xi}) \propto \exp \left(-\xi^{2} / 2\right)$, i.e., I introduce the double average,

$$
\left\langle\langle A ; t\rangle=\int d^{3} \xi P(\boldsymbol{\xi}) \int d \Gamma \varrho(\Gamma, t) A(\Gamma) .\right.
$$

While the driving does not change the density directly, $i \mathcal{L}_{D}^{+} \varrho_{q}=0$, matrix elements with the current vanish, because the kicks are uncorrelated with the particles' velocities, $\left\langle\left\langle\boldsymbol{j}_{q} \mid \mathcal{L}_{D}^{+} \boldsymbol{j}_{q}\right\rangle=0\right.$.

The formally exact form of the granular YBG theorem reads

$$
\begin{aligned}
n \int d^{3} r_{3} g_{3}\left(\boldsymbol{r}_{1}, \boldsymbol{r}_{2}, \boldsymbol{r}_{3}\right)[ & \left.\delta\left(r_{13}-2 a\right) \hat{\boldsymbol{r}}_{1}+\nabla_{1} \ln \vartheta\left(r_{13}\right)\right] \\
& =\nabla_{1} g\left(r_{12}\right)-\chi \delta\left(r_{12}-2 a\right) \hat{\boldsymbol{r}}_{1}+g\left(r_{12}\right) \nabla_{1} \ln \vartheta\left(r_{12}\right) .
\end{aligned}
$$

To be able to use the YBG theorem in the form for elastic hard spheres [eq. 2.25]], I make the nontrivial assumption,

$$
g\left(r_{12}\right) \nabla_{1} \ln \vartheta\left(r_{12}\right) \approx n \int d^{3} r_{3} g_{3}\left(\boldsymbol{r}_{1}, \boldsymbol{r}_{2}, \boldsymbol{r}_{3}\right) \nabla_{1} \ln \vartheta\left(r_{13}\right) .
$$

\subsection{Perspectives}

While the velocity distribution function of the driven granular fluid is relatively well understood (see, Ref. [208] but also Ref. [209]), it would be good to have a more detailed characterization of the spatial distribution function that goes beyond the approach of treating it as identical to that of an elastic hard sphere fluid.

An explicit derivation of the adjoint pseudo Liouville operator, $\mathcal{L}_{+}^{\dagger}$, in the driven dissipative case is work in progress. Preliminary results show that its knowledge will probably not simplify any calculations but I expect that it will allow new insights into the dynamics of the granular fluid. For some established mathematical results concerning granular dynamics, see the review by Villani [210]. 


\section{The Static Structure}

While the following chapters will be concerned with dynamic correlation functions, in this chapter I want to discuss the static structure of a driven granular fluid. Besides the static structure factor, $S(k)=N\left\langle\rho_{q} \rho_{-q}\right\rangle$, I will focus on the current correlator, $S_{j j}(k)=N\left\langle\boldsymbol{j}_{q} \boldsymbol{j}_{-q}\right\rangle$, where, $m \boldsymbol{j}(\boldsymbol{r})=m n \boldsymbol{u}$, is the momentum current. The correlations of the temperature field, $T(\boldsymbol{r})$, with the particle density, $\rho(\boldsymbol{r})$, and momentum current, $\boldsymbol{j}(\boldsymbol{r})$, will also be considered briefly.

One of the first observations will be that the static structure of driven granular fluid qualitatively resembles that of a fluid in thermal equilibrium - at least as long as the driving conserves momentum. If this is not the case, one observes a divergence, $S(k \rightarrow 0) \propto k^{-2}$, for small wave numbers $k$. Equivalent formulations of this feature predict long range correlations, $g(r \rightarrow \infty)-1 \propto r^{-1}$, in real space and giant number fluctuations, $\delta N^{2}=\left\langle N^{2}\right\rangle-\langle N\rangle^{2} \propto N^{\alpha}$, with $\alpha>1$. In fact an argument adapted from Refs. [121] and [67] predicts $\delta N \propto N^{2 / 3} \ln N$, i.e., $\alpha=4 / 3$ up to logarithmic corrections.

An explicit derivation of this result within a theory of fluctuating hydrodynamics will be given below. Alternatively, it can be understood from results in the study of self organized criticality. The sand pile model, which was used to introduce the concept of self organized criticality [72, 211] is a lattice model. A corresponding continuum description was developed by Hwa and Kardar [212. With this model in mind Grinstein et al. [213, 214] studied the correlation function $C(k) \propto\left\langle h_{k} h_{-k}\right\rangle$ of an observable $h$ obeying the stochastic equation of motion,

$$
\partial_{t} h=\eta \nabla^{2} h+\xi,
$$

with a stochastic force $\xi$ with zero mean, $\langle\xi\rangle=0$, and variance $\left\langle\xi(k, t) \xi\left(-k, t^{\prime}\right)\right\rangle=$ $2 D(k) \delta\left(t-t^{\prime}\right)$. Then the correlation function is essentially given by $C(k) \propto D(k) / \eta k^{2}$ which leads to a $k^{-2}$ divergence if $D(k)=$ const. as in the continuum sandpile model. If $h$ were a macroscopic observable of a system in thermal equilibrium, then a fluctuation dissipation theorem would require that $D(k) \propto \eta k^{2}$ and consequentially, $C(k \rightarrow 0)=$ const. Moreover, in this case $C(k \rightarrow 0)$ is completely independent of the transport coefficient $\eta$. The analysis of Grinstein et al. also showed that this argument remains valid even if the equations of motion is modified by additional terms from a large class of functional forms. Therefore, the same small $k$ behavior can be predicted for the hydrodynamic correlation functions including the static structure factor $S(k)$. 
While the equilibrium fluctuation dissipation theorems make it impossible to extract the transport coefficients like the viscosity or the heat conductivity from the static correlation functions of a fluid in thermal equilibrium, the speed of sound, $c$, is related to the static structure factor, $c^{2}=\lim _{k \rightarrow 0} T / m S(k)$. I will show below, that this can be generalized for the driven granular fluid in the form $c^{2}=\lim _{k \rightarrow 0} S_{\ell \ell}(k) / S(k)$, where $S_{\ell \ell}(k)$ is the longitudinal current correlator.

The noise induced by the external stochastic driving force is not restricted by an equilibrium fluctuation dissipation theorem. I will show below that this makes it possible to measure the transport coefficients of a driven granular fluid from the static correlation functions in the case of naive volume driving. In particular, I will use the current correlator, $S_{j j}(k)$, to measure the shear viscosity, $\nu_{\perp}$, and the sound damping constant, $D_{S}$, from its transverse, $S_{\perp \perp}(k) \propto 1 / \nu_{\perp} k^{2}$, and longitudinal part, $S_{\ell \ell}(k) \propto 1 / D_{S} k^{2}$, respectively.

In the next section, I will develop a fluctuating hydrodynamic theory based on the work in Ref. [74. In section 3.2. I will present details of the event driven simulations performed to study the driven granular fluid as well as of the way I measured the static correlation functions. The results, including the extracted quantities: speed of sound, $c$, sound damping constant, $D_{S}$, and shear viscosity, $\nu_{\perp}$, will be discussed in section 3.3

\subsection{Fluctuating Hydrodynamics}

\subsubsection{Hydrodynamic Equations}

Either from rederiving it starting from the Boltzmann equation [215]217] or by analogy one can write down the following set of balance equations for a homogeneous freely cooling inelastic granular gas,

$$
\begin{gathered}
D_{t} n+n \nabla \cdot \boldsymbol{u}=0 \\
D_{t} u_{i}+\frac{1}{m n} \nabla_{j} \mathrm{P}_{i j}=0 \\
D_{t} T+\frac{2}{3 n}\left(\mathrm{P}_{i j} \nabla_{j} u_{i}+\nabla \cdot \boldsymbol{q}\right)=-\Gamma .
\end{gathered}
$$

These are the continuity equations for the number density, $n$, the flow velocity, $\boldsymbol{u}$, and the granular temperature, $T$. Einstein summation convention is understood and $D_{t}=\partial_{t}+\boldsymbol{u} \cdot \nabla$ denotes the material derivative. Dissipation gives rise to the energy sink term $\Gamma$. Within the Enskog approximation and neglecting the deviations of the velocity distribution function from a gaussian on finds $\Gamma \approx \Gamma_{E}=\gamma \omega_{E} T$ where $\omega_{E}$ is the elastic Enskog collision frequency and $\gamma=\left(1-\epsilon^{2}\right) / 3$ contains the coefficient of restitution [99]. 
To include the driving force into the hydrodynamic description, the random force, $\boldsymbol{\xi}_{i}(t)$, acting on individual particles has to be promoted to a random field,

$$
\boldsymbol{\xi}(\boldsymbol{r}, t)=\frac{1}{n} \sum_{i} \boldsymbol{\xi}_{i}(t) \delta\left(\boldsymbol{r}-\boldsymbol{r}_{i}(t)\right)
$$

with variance

$$
\left\langle\xi^{\alpha}\left(\boldsymbol{r}_{i}, t\right) \xi^{\beta}\left(\boldsymbol{r}_{j}, t^{\prime}\right)\right\rangle_{\xi}=\frac{1}{n} \xi_{0}^{2}\left(r_{i j}\right) \delta^{\alpha \beta} \delta\left(t-t^{\prime}\right) .
$$

For the temperature or energy equation there is both an average contribution, $m \xi_{0}^{2} \equiv m \xi_{0}^{2}(r=0)$, as well as a fluctuating field,

$$
\vartheta(\boldsymbol{r}, t)=\frac{2 m}{3 n} \sum_{i}\left[\boldsymbol{v}_{i} \cdot \boldsymbol{\xi}_{i}(t)\right] \delta\left(\boldsymbol{r}-\boldsymbol{r}_{i}(t)\right)
$$

with variance

$$
\left\langle\vartheta\left(\boldsymbol{r}_{i}, t\right) \vartheta\left(\boldsymbol{r}_{j}, t^{\prime}\right)\right\rangle_{\xi}=\frac{4 m T}{3 n} \xi_{0}^{2}\left(r_{i j}\right) \delta\left(t-t^{\prime}\right) .
$$

Put together, one arrives at a set of stochastic equations [74],

$$
\begin{aligned}
D_{t} n+n \nabla \cdot \boldsymbol{u} & =0 \\
D_{t} u_{i}+\frac{1}{m n} \nabla_{j} \mathrm{P}_{i j} & =\xi_{i} \\
D_{t} T+\frac{2}{3 n}\left(\mathrm{P}_{i j} \nabla_{j} u_{i}+\nabla \cdot \boldsymbol{q}\right) & =m \xi_{0}^{2}-\Gamma+\vartheta .
\end{aligned}
$$

Asymptotically, the energy input from driving will balance the dissipation, $m \xi_{0}^{2}-$ $\Gamma \rightarrow 0$, and the system is characterized by a mean density, $n_{0}$, and a mean temperature, $T_{0}$. Moreover, let us assume that the mean flow velocity vanishes, $\boldsymbol{u}_{0} \equiv \mathbf{0}$. On the Enskog level, one finds

$$
\frac{T_{0 E}}{m}=\left[\frac{\sqrt{\pi} a \xi_{0}^{2}}{4\left(1-\epsilon^{2}\right) \chi \varphi}\right]^{2 / 3} .
$$

The main assumption of hydrodynamic theory is that the balance equations 3.2 can be closed by expanding the stress tensor, $\mathrm{P}$, and the heat flux, $\boldsymbol{q}$, in gradients of the hydrodynamic fields, $n, \boldsymbol{u}$ and $T$. To first, or Navier-Stokes order, this expansion reads for an homogeneous and isotropic fluid [115,

$$
\begin{aligned}
\mathrm{P}_{i j} & \simeq p \delta_{i j}-\eta\left(\nabla_{i} u_{j}+\nabla_{j} u_{i}-\frac{2}{3} \delta_{i j} \nabla_{k} u_{k}\right)-\zeta \delta_{i j} \nabla_{k} u_{k}, \\
\boldsymbol{q} & \simeq-\kappa \nabla T .
\end{aligned}
$$

The pressure, $p$, is related to the hydrodynamic fields via the equation of state: $p(n, T)=n T(1+4 \varphi \chi)$ for elastic hard spheres [48]. The contact value, $\chi(n)$, of 
the pair correlation function, $g(r)$, is a function of density as well. The remaining parameters are the shear and bulk viscosity, $\eta$ and $\zeta$, respectively, and the heat conductivity $\kappa$. A peculiar contribution to the heat flux $\propto \nabla n$ that is specific to granular gases and absent in elastic fluids [218] is neglected. For the case of a driven granular fluid, it was found to be extremely small anyway [219].

\subsubsection{Linearzation and Relaxation Rates}

If we linearize the hydrodynamic equations around this homogeneous state in the fields $n, \boldsymbol{u}, T$ and Fourier transform both the spatial as well as the time coordinate, we can write the hydrodynamic equations as a set of linear equations for the deviations from the homogeneous state:,

$$
i \omega \delta \boldsymbol{a}(\boldsymbol{k}, \omega)=\mathrm{M}(\boldsymbol{k}) \delta \boldsymbol{a}(\boldsymbol{k}, \omega)+\boldsymbol{f}^{\mathrm{dr}}(\boldsymbol{k}),
$$

where $\delta \boldsymbol{a}=\left(\delta n, \delta T, u_{\ell}, \boldsymbol{u}_{\perp}\right)$ is the vector of deviation fields, $\delta n(\boldsymbol{r})=n(\boldsymbol{r})-n_{0}$, $\delta T(\boldsymbol{r})=T(\boldsymbol{r})-T_{0}$ and the flow velocity has been decomposed into the longitudinal part, $u_{\ell}(\boldsymbol{k})=\hat{\boldsymbol{k}} \cdot \boldsymbol{u}(\boldsymbol{k})$, and the transverse part, $\boldsymbol{u}_{\perp}(\boldsymbol{k})=\boldsymbol{u}-\hat{\boldsymbol{k}} u_{\ell}(\boldsymbol{k})$. The hydrodynamic matrix,

$$
\mathrm{M}(\boldsymbol{k})=-\left(\begin{array}{ccccc}
0 & 0 & i k n & 0 & 0 \\
g(n) \Gamma / n & D_{T} k^{2}+3 \Gamma / 2 T & 2 i k p / 3 n & 0 & 0 \\
i k c_{T}^{2} / n & i k p / m n T & \nu_{l} k^{2} & 0 & 0 \\
0 & 0 & 0 & \nu_{\perp} k^{2} & 0 \\
0 & 0 & 0 & 0 & \nu_{\perp} k^{2}
\end{array}\right),
$$

is given in terms of the thermal diffusivity, $D_{T}=2 \kappa / 3 n$, the isothermal sound velocity, $c_{T}^{2}=\partial p / \partial m n$, and $\nu_{\ell}=(4 \eta / 3+\zeta) / m n, \nu_{\perp}=\eta / m n$ the longitudinal and transverse viscosity, respectively. The term $g(n)=1+n \partial \ln \chi / \partial n$ is the linearized equation of state. In an elastic gas the $M_{n T}$ term would be zero and the $M_{T T}$ term would come without the $k$-independent part. Note that the equation for the transverse velocity, $\boldsymbol{u}_{\perp}$, is decoupled form the rest of the equations as in elastic fluids. The vector of driving forces, $\boldsymbol{f}^{\mathrm{dr}}=\left(0, \vartheta, \xi_{\ell}, \boldsymbol{\xi}_{\perp}\right)$, contains all stochastic terms, where $\xi_{\ell}=\hat{\boldsymbol{k}} \cdot \boldsymbol{\xi}$ and $\boldsymbol{\xi}_{\perp}=\boldsymbol{\xi}-\hat{\boldsymbol{k}} \xi_{\ell}$.

The eigenvalues, $z_{\lambda}(\boldsymbol{k}) \equiv i \omega_{\lambda}(\boldsymbol{k})$, of the hydrodynamic matrix determine the principal macroscopic relaxation rates. In an inelastic system, McNamara [52] identified a critical length scale or wave number, $k_{*}$, which separates the elastic regime, $k \gg k_{*}$, where relaxation rates are that of an elastic system from the dissipative regime, $k \ll k_{*}$, where the dissipation of energy is completely dominated by the inelastic collisions.

From the hydrodynamic matrix, $\mathrm{M}(k)$, one finds that the critical wave number, $k_{*}$, is given by (see appendix C.1)

$$
\left(D_{T}+\nu_{\ell}\right) k_{*}^{2}=3 \Gamma / 2 T .
$$


Values of $k_{*}$ for the parameters of the simulations and with expressions for $D_{T}$ and $\nu_{\ell}$ from Ref. [219] and $\Gamma=\Gamma_{E}$ are given in table 3.2 .

The relaxation rate of the shear mode can immediately be seen to be given by

$$
z_{\perp}=-\nu_{\perp} k^{2}
$$

For the other eigenvalues one finds to second order in $k$ in the elastic regime (see also Ref. [115])

$$
\begin{aligned}
& z_{H} \simeq-D_{H} k^{2}, \\
& z_{ \pm} \simeq \mp i c_{>} k-D_{S}^{>} k^{2},
\end{aligned}
$$

where $c_{>}$is the adiabatic speed of sound, $c_{>}^{2}=\gamma_{\mathrm{ad}} c_{T}^{2} \equiv c_{T}^{2}+2 p^{2} / 3 m n^{2} T$, with the adiabatic index, $\gamma_{\text {ad }}$. The sound damping, $2 D_{S}^{>}=\nu_{\ell}+\left(\gamma_{\text {ad }}-1\right) D_{T} / \gamma_{\text {ad }}$, includes a contribution from the thermal diffusivity and $D_{H}=D_{T} / \gamma_{\text {ad }}$.

In the dissipative regime (see appendix C.1, heat diffusion is irrelevant but there are still propagating sound waves,

$$
\begin{aligned}
& z_{H}=-3 \Gamma / 2 T, \\
& z_{ \pm} \simeq \mp i c_{<} k-D_{S}^{<} k^{2},
\end{aligned}
$$

with a speed of sound,

$$
c_{<}^{2}=c_{T}^{2}-2 g p / 3 m n,
$$

different from the elastic speed of sound, $c_{>}$. In general, the speed of sound in the inelastic fluid is considerably smaller than in the corresponding elastic system. In the limit of vanishing density, $n \rightarrow 0, p=n T$ and $g=1$, while $\gamma_{\text {ad }}=5 / 3$ for spherical particles. Then one finds $c_{>}^{2}=\frac{5}{3} c_{T}^{2}=5 c_{<}^{2}$.

The sound damping constant, $2 D_{S}^{<}=\nu_{\ell}+D_{\Gamma}$, is independent of the thermal conductivity and contains the new transport coefficient,

$$
D_{\Gamma}=\frac{4 p T}{9 m n \Gamma}(g+p / n T),
$$

instead. For low densities, $D_{\Gamma}=\frac{8}{9} \frac{T}{m} \frac{T}{\Gamma}$. The thermal diffusivity in the elastic regime can be written as $D_{T}=C \frac{T}{m} \omega_{0}^{-1}$ with a constant, $C$, of the order one. For the transport coefficient $D_{\Gamma}$, the collision frequency, $\omega_{0}$, is replaced by the cooling rate, $\Gamma / T$. The sound damping constant, $D_{S}^{<}$, diverges in the elastic limit, $\epsilon \rightarrow 1$, but as the length scales necessary to observe the inelastic regime also diverges, the relaxation rate $D_{S}^{<} k^{2} \ll D_{S}^{<} k_{*}^{2}=\frac{p}{3 m n} \frac{g+p / n T}{D_{T}+\nu_{\ell}}<\infty$ remains finite.

The relaxation rates given by the equations $(3.13)-(3.17)$ confirm the results of Ernst et al. [74] except for an additional heat diffusion term that was consistently dropped here. Both in the elastic as well as in the inelastic regime, all eigenvalues 
are negative implying that the homogeneous state is at least linearly stable [52]. The intuitive expectation that driving the fluid will avoid the clustering instability is thus confirmed.

As the hydrodynamic matrix, $\mathrm{M}$, is not symmetric, it has both right eigenvectors, $\mathrm{M}(\boldsymbol{k}) \boldsymbol{w}^{\lambda}(\boldsymbol{k})=z_{\lambda}(\boldsymbol{k}) \boldsymbol{w}^{\lambda}(\boldsymbol{k})$, and left eigenvectors, $\boldsymbol{v}^{\lambda}(\boldsymbol{k}) \mathrm{M}(\boldsymbol{k})=\boldsymbol{v}^{\lambda}(\boldsymbol{k}) z_{\lambda}(\boldsymbol{k})$ to the eigenvalue $z_{\lambda}(\boldsymbol{k})$. To fix a normalization, we require $\sum_{\lambda} \boldsymbol{w}^{\lambda} \boldsymbol{v}^{\lambda}=\mathrm{I}$ with the identity I. Besides the trivial result $\boldsymbol{w}^{\perp}=\boldsymbol{v}_{\perp}=(0,0,0,1)$, one finds to zeroth order in $k$ and in the dissipative regime

$$
\begin{aligned}
& \boldsymbol{w}^{H} \simeq(0,1,0,0), \\
& \boldsymbol{w}^{ \pm} \simeq\left(1,-2 g T / 3 n, \pm c_{<} / n, 0\right)
\end{aligned}
$$

and

$$
\begin{aligned}
\boldsymbol{v}_{n} & \simeq(2 \sqrt{2} g T / 3 n, 1,1,0) / \sqrt{2}, \\
\boldsymbol{v}_{T} & \simeq(1,0,0,0), \\
\boldsymbol{v}_{\ell} & \simeq\left(0, n / c_{<},-n / c_{<}, 0\right),
\end{aligned}
$$

in agreement with Ref. [74]. I give the duals of the left eigenvectors, $\boldsymbol{v}^{\lambda}$, as they are needed below. Note that the sound modes $w^{ \pm}$couple to the temperature mode. This is not the case in an equilibrium fluid.

\subsubsection{Fluctuating Hydrodynamics}

In the linear regime of small fluctuations $\delta \boldsymbol{a}$, the terms neglected in expansion of the pressure tensor and the heat flux can be described by random variables, $\pi_{i j}$ and $\boldsymbol{g}$ (see, e.g. Refs. [49] and [122, 220]),

$$
\begin{aligned}
\mathrm{P}_{i j} & \simeq p \delta_{i j}-\eta\left(\nabla_{i} u_{j}+\nabla_{j} u_{i}-\frac{2}{3} \delta_{i j} \nabla_{k} u_{k}\right)-\zeta \delta_{i j} \nabla_{k} u_{k}+\pi_{i j}, \\
\boldsymbol{q} & \simeq-\kappa \nabla T+\boldsymbol{g} .
\end{aligned}
$$

It turns out to be more convenient to work with the variables $\Xi_{i}=\nabla_{j} \pi_{i j} / \mathrm{mn}$ and $\Theta=2 \nabla \cdot \boldsymbol{g} / 3 n$, which can then be combined into a vector of internal noise, $f^{\text {int }}=\left(0, \Theta, \Xi_{\ell}, \boldsymbol{\Xi}_{\perp}\right)$, where $\Xi_{\ell}$ and $\boldsymbol{\Xi}_{\perp}$ are defined as usual. The internal noise has zero mean, $\left\langle\boldsymbol{f}^{\text {int }}\right\rangle=0$, by construction. For a fluid in thermal equilibrium, one can express the variance of $\boldsymbol{f}^{\text {int }}$ in terms of the transport coefficients [220]: For the temperature fluctuations one finds

$$
\langle\theta(\boldsymbol{k}, t) \theta(-\boldsymbol{k}, t)\rangle_{\Theta}=\frac{4 T}{3 n} D_{T} V k^{2},
$$

while the velocity fluctuations take the following form,

$$
\left\langle\Xi_{i}(\boldsymbol{k}, t) \Xi_{j}(-\boldsymbol{k}, t)\right\rangle_{\Xi}=\frac{2 T}{m n} V k^{2}\left[\nu_{\perp}\left(\delta_{i j}-\hat{k}_{i} \hat{k}_{j}\right)+\nu_{l} \hat{k}_{i} \hat{k}_{j}\right] .
$$


In the terminology of Toda et al. [221, the two relations above are instances of the fluctuation dissipation theorem (FDT) of the second kind.

The distribution function of a driven granular fluid is known to be not a simple Boltzmann factor, $\exp (-\beta H)$, and thus the equilibrium FDTs do not hold. The question of what determines the amplitudes of the internal fluctuations in a driven granular fluid is beyond the scope of this thesis. First results have been obtained recently by Maynar et al. [222]. They found that under some assumptions the equilibrium FDT still applies for the transverse component of flow velocity. Here, I will rely on heuristic reasoning instead.

The tensorial structure of the variances follows from the assumption of homogeneity and isotropy that are the same whether we consider an equilibrium or a non-equilibrium fluid. This also requires that the variances are a function of $k^{2}$. Neglecting the possibility of a nonanalyticity at $k=0$, we expect that the variances of the internal noise are of the same functional form as for fluids in thermal equilibrium but will be parametrized by constants $D_{T}^{\text {int }}, \nu_{\ell}^{\text {int }}$ and $\nu_{\perp}^{\text {int }}$ different from the heat conductivity, $\kappa$, and the viscosities $\nu_{\ell}$ and $\nu_{\perp}$. A wave number independent term can be ruled out because the statistical fluctuations vanish in the thermodynamic limit. At this point the question whether the fluctuations, quantified by, e.g., $\nu_{\perp}^{\text {int }}$, are completely determined by the parameters quantifying dissipation is still unresolved. If the answer were negative, the relation that determines the strength of the fluctuations should probably not be called a fluctuation dissipation theorem.

Fluctuations around the homogeneous state are thus excited both by the driving force, $f^{\mathrm{dr}}$, and by the internal noise, $\boldsymbol{f}^{\mathrm{int}}$,

$$
i \omega \delta \boldsymbol{a}(\boldsymbol{k}, \omega)=\mathrm{M}(\boldsymbol{k}) \delta \boldsymbol{a}(\boldsymbol{k}, \omega)+\boldsymbol{f}(\boldsymbol{k}),
$$

where, $\boldsymbol{f}=\boldsymbol{f}^{\mathrm{dr}}+\boldsymbol{f}^{\mathrm{int}}$, is a vector of random variables with zero mean, $\langle\boldsymbol{f}\rangle=0$, and variance $\left\langle f_{i}(\boldsymbol{k}, t) \mid f_{j}\left(\boldsymbol{p}, t^{\prime}\right)\right\rangle=\Gamma_{i j}(k) V \delta(\boldsymbol{k}-\boldsymbol{p}) \delta\left(t-t^{\prime}\right)$. The matrix of variances, $\Gamma$, has the following nonzero entries

$$
\begin{gathered}
\frac{3}{4} n \Gamma_{T T}(k)=T m \xi_{0}^{2}(k)+D_{T}^{\mathrm{int}} T^{2} k^{2} \\
n \Gamma_{\ell \ell}(k)=\xi_{0}^{2}(k)+\frac{2 \nu_{\ell}^{\mathrm{int}} T}{m} k^{2} \\
n \Gamma_{\perp \perp}(k)=\xi_{0}^{2}(k)+\frac{2 \nu_{\perp}^{\text {int }} T}{m} k^{2} .
\end{gathered}
$$

\subsubsection{Static Correlation Functions}

Based on the linearized fluctuating hydrodynamic equations we can now derive an expression for the static structure factor, $\Sigma_{a b}(\boldsymbol{k})$, (see appendix C.2)

$$
\Sigma_{a b}(\boldsymbol{k})=-\sum_{\lambda, \mu \in \mathcal{H}} \frac{w_{a}^{\lambda}(\boldsymbol{k}) w_{b}^{\mu}(-\boldsymbol{k})}{z_{\lambda}(k)+z_{\mu}^{*}(k)} \sum_{c \in \mathcal{N}} v_{c}^{\lambda}(\boldsymbol{k}) \Gamma_{c c}(k) v_{c}^{\mu}(-\boldsymbol{k}),
$$


with the index sets $\mathcal{H}=\{H, \pm, \perp\}$ and $\mathcal{N}=\{n, T, \ell, \perp\}$.

In the limit of small wave number one finds the following nonzero results. Apart from the transverse current correlator,

$$
S_{\perp \perp}(k)=n \Sigma_{\perp \perp}(k) \simeq \frac{n \Gamma_{\perp \perp}(k)}{2 \nu_{\perp} k^{2}}
$$

and the two-point temperature correlation function,

$$
S_{T T}(k)=n \Sigma_{T T}(k) \simeq \frac{4 g^{2} T^{2}}{9 c_{<}^{2}} S_{\ell \ell}(k)+\frac{T n \Gamma_{T T}(k)}{3 m \xi_{0}^{2}},
$$

all other correlation functions are proportional to the longitudinal current correlator,

$$
S_{\ell \ell}(k)=n \Sigma_{\ell \ell}(k) \simeq \frac{n \Gamma_{\ell \ell}(k)}{2 D_{S}^{<} k^{2}} .
$$

The most interesting is most likely the static structure factor,

$$
S(k)=\frac{1}{n} \Sigma_{n n}(k) \simeq S_{\ell \ell}(k) / c_{<}^{2} .
$$

In particular this relation will be used below to measure the speed of sound as $c_{<}^{2}=\lim _{k \rightarrow 0} S_{\ell \ell}(k) / S(k)$. Sound propagation relies on the effect that density and current are $90^{\circ}$ out of phase,

$$
S_{n \ell}(k)=n \Sigma_{n \ell}(k) \simeq i \frac{D_{S}^{<} k}{c_{<}^{2}} S_{\ell \ell}(k)
$$

while the result that density and temperature fluctuations are anticorrelated,

$$
S_{n T}(k)=n \Sigma_{n T}(k) \simeq-\frac{2 g n T}{3 c_{<}^{2}} S_{\ell \ell}(k)
$$

is a peculiar effect of granular fluids. In a freely cooling granular gas, this leads to the clustering instability [52]. For an equilibrium fluid one can show that $S_{n T}(k \rightarrow$ $0) \equiv 0[223$.

The last nonzero cross-correlator is

$$
S_{T \ell}(k)=n \Sigma_{T \ell}(k) \simeq i \frac{2 g T D_{S}^{<} k}{3 c_{<}^{2}} S_{\ell \ell}(k) .
$$

The purely imaginary correlators $S_{n \ell}(k)$ and $S_{T \ell}(k)$ have been ignored in Ref. [74].

At this point the origin of the small $k$ divergence becomes apparent. The two important correlation functions $S_{\perp \perp}(k)$ and $S_{\ell \ell}(k)$ are of the form $S_{a a}(k) \propto \Gamma_{a a}(k) / k^{2}$. For an equilibrium fluid, $\Gamma_{a a}(k) \propto k^{2}$, such that all the correlation functions tend to a 
finite value, $S_{a a}(k \rightarrow 0)=$ const., but for the naive volume driving, $n \Gamma_{a a}(k \rightarrow 0)=\xi_{0}^{2}$, which leads to the $k^{-2}$ divergence of the correlation functions.

If the driving conserves momentum on a length scale $\ell$, we have

$$
\xi_{0}^{2}(k)=\xi_{0}^{2}[1-\operatorname{sinc}(k \ell)] \simeq \xi_{0}^{2} \ell^{2} k^{2}
$$

and thus, $\Gamma_{a a}(k) \propto k^{2}$, again like for an equilibrium fluid. This relation between momentum conservation and the small $k$ behavior of the static correlation functions is one of the main results of this chapter.

Let us first consider the driving that does not respect momentum conservation, $\xi_{0}^{2}(k) \equiv \xi_{0}^{2}$. Then one finds

$$
S_{\perp \perp}(k) \simeq \frac{\xi_{0}^{2}}{2 \nu_{\perp} k^{2}}+\frac{\eta^{\text {int }}}{\eta} \frac{T}{m}
$$

which can be used to measure the shear viscosity, $\nu_{\perp}$, and the ratio $\eta^{\text {int }} / \eta$ which quantifies the FDT violation. From the longitudinal current correlator,

$$
S_{\ell \ell}(k) \simeq \frac{\xi_{0}^{2}}{2 D_{S}^{<} k^{2}}+\frac{\nu_{\ell}^{\text {int }}}{D_{S}^{<}} \frac{T}{m}
$$

I will extract the sound damping constant $D_{S}^{<}$.

For the locally momentum conserving driving, one finds

$$
S_{\perp \perp}(k) \simeq \frac{\xi_{0}^{2} \ell^{2}}{2 \nu_{\perp}}+\frac{\eta^{\text {int }}}{\eta} \frac{T}{m}
$$

i.e., a sum of two terms where $\nu_{\perp}$ and $\eta^{\text {int }} / \eta$ are the unknowns. One the one hand, this makes it impossible to extract the shear viscosity from measurements of the transverse current correlator. On the other hand, eq. (3.36) above can be rewritten as

$$
\tilde{\nu}_{\perp}^{\text {int }}=\tilde{\nu}_{\perp} \frac{m}{T} S_{\perp \perp}(k \rightarrow 0)-\frac{\gamma(\epsilon)}{2}
$$

in terms of the dimensionless viscosity, $\tilde{\nu}_{\perp}=\nu_{\perp} / \omega_{0} \ell^{2}$, The measurements described below are compatible with $m S_{\perp \perp}(k \rightarrow 0) / T=1$, independent of density $\varphi$ and dissipation $\epsilon$ (see Fig. 3.8). Therefore I propose the following FDT for the granular fluid with locally momentum conserving driving,

$$
\tilde{\nu}_{\perp}^{\text {int }}=\tilde{\nu}_{\perp}-\frac{\gamma(\epsilon)}{2},
$$

which smoothly connects to the equilibrium result, $\nu_{\perp}^{\mathrm{int}} \equiv \nu_{\perp}$. Fluctuations of the internal transverse random force, $\boldsymbol{\xi}_{\perp}$, are thus smaller in the driven granular fluid compared to a fluid in thermal equilibrium 
From the longitudinal current correlator,

$$
S_{\ell \ell}(k) \simeq \frac{\xi_{0}^{2} \ell^{2}}{2 D_{S}^{<}}+\frac{\nu_{\ell}^{\text {int }}}{D_{S}^{<}} \frac{T}{m},
$$

and the observation that it appears to tend toward an universal value, $m S_{\ell \ell}(k \rightarrow$ 0 ) $/ T \approx 0.5$ (see Fig. 3.6), one can derive another FDT,

$$
2 \tilde{\nu}_{\ell}^{\text {int }}=\frac{1}{2}\left(\tilde{\nu}_{\ell}+\tilde{D}_{\Gamma}\right)-\gamma(\epsilon) .
$$

In contrast to the transverse random force, the fluctuations of the longitudinal random force, $\xi_{\ell}$, in the driven granular fluid are not smoothly connected to the fluctuations in thermal equilibrium. Instead, they diverge in the elastic limit, $\epsilon \rightarrow 1$. As any resulting large current is quickly damped away by the divergent sound damping, $D_{S}^{<}$, this does not necessarily lead to large current fluctuations.

\subsection{Simulations}

\subsubsection{Event Driven Molecular Dynamics and Volume Driving}

Event driven [224] simulations were performed using an existing program that was modified to allow for the locally momentum conserving driving. To resolve the small $k$ behavior, the system size should be as large as possible. At the time of the first simulations, the maximum number of particles that could be accomodated by the main memory was $N=400000$. The runtime of the simulations was mainly limited by the maximum permissible CPU time of 28 days per job.

Whether an inelastic collapse can occur in driven system is still a controversial issue [225, 226]. A number of techniques has been proposed to avoid the inelastic collapse by using modified collision rules [227, 228]. Here, we use the method of virtual hulls [67] with a thickness of $\Delta r / 2 a=10^{-5}$. I believe that this is one of the least intrusive methods to treat the inelastic collapse.

Despite what we assume for the theoretical description of the momentum conserving driving mechanism, it is impossible to find a partner particle at a distance $\ell$, exactly. Apart from completely artificial situations, the probability for two particles to be any given distance apart is always zero. Thus, for the simulation, we have to allow for some range of distances for finding a partner particle. What is done here, is to start at a given distance, $\ell_{\min }$, and search for the first particle, $j$, such that its distance from the particle $i$ is at least $\ell_{\min }$, i.e,

$$
j=\underset{k}{\arg \min }\left\{r_{i k} \geq \ell_{\min }\right\} .
$$

This will give rise to a distribution, $P(\ell)$, of pair distances chosen for a driving pair with a well defined mean, $\bar{\ell}$. The mean driving distance, $\bar{\ell}$, of the simulated systems 
Table 3.1: Mean driving distance, $\bar{\ell} / a$, as a function of volume fraction, $\varphi$, and coefficient of restitution, $\epsilon$.

\begin{tabular}{ccccc}
\hline \multirow{2}{*}{ Restitution $\epsilon$} & \multicolumn{4}{c}{ Volume Fraction $\varphi$} \\
\cline { 2 - 5 } & 0.1 & 0.2 & 0.3 & 0.4 \\
\hline 0.9 & 2.439 & 2.157 & 2.098 & 2.051 \\
0.8 & 2.433 & 2.153 & 2.095 & 2.049 \\
0.7 & 2.429 & 2.149 & 2.092 & 2.046 \\
\hline
\end{tabular}

is shown in table 3.1. Note the small but significant decrease in $\bar{\ell}$ with increasing dissipation.

To keep the center of mass momentum, $\boldsymbol{P}(t)$, constant throughout a simulation run, pairs of particles were kicked simultaneously even for the naive volume driving. For that purpose, random pairs of particles were determined at the start of the simulation. These pairs remained unchanged throughout the simulation. One can show [229] that this leads to corrections in the long wavelength behavior of the structure factor on the order of $1 / N$. In particular, a renormalized structure factor, $\tilde{S}(k)=S(k) /[1+S(k) / N]$, is measured. Within the error bars, it will be indistinguishable, $\tilde{S}(k) \approx S(k)$.

The driving strength, $p_{\mathrm{dr}}$, was determined within the Enskog approximation such that the driving frequency, $\omega_{\mathrm{dr}}$, equals the Enskog collision frequency, $\omega_{E}$ (see also Ref. [110]). Using a higher driving frequency, $\omega_{\mathrm{dr}}=10 \omega_{E}$, for a subset of the initial conditions did not show significant differences. The random numbers needed for the driving were generated by the RANMAR algorithm [230].

\subsubsection{Initial Conditions and Simulation Parameters}

Initial configurations were generated by the following method. The system size, $L$, for $N=400000$ particles corresponding to a given volume fraction, $\varphi=\frac{4}{3} \pi N(a / L)^{3}$, was determined and then 400 particles where placed in a box of size $L / 10$ by random sequential addition 1 . If this did not succeed within a reasonable time, the run was canceled and restarted with a different random seed. Once the random positions for the small system had been determined, the complete system was tiled with copies of the subsystem. Subsequently, $N-1$ random velocities were drawn from a gaussian distribution with unit varianc $\mathrm{f}^{2}$ and assigned to all but one of the particles. The velocity of the last particle was chosen such that the system has vanishing total

\footnotetext{
${ }^{1}$ A Mersenne Twister pseudo random number generator [231] in the implementation by SciPy 232 ] was used. As the hard sphere dynamics is chaotic (see, e.g., Ref. 233), I expect that the results are independent of the pseudo random number generator.

${ }^{2}$ Generated by the common Box-Muller transformation [234].
} 
Table 3.2: Number of initial conditions, $M$, per combination of volume fraction, $\varphi$, and coefficient of restitution, $\epsilon$; corresponding minimal wave number, $2 k_{\min } a$, for $N=400000$ particles and critical wave number, $2 k_{*} a$ [see eq. 3.12 ]

\begin{tabular}{ccccc}
\hline$\varphi$ & $\epsilon$ & $M$ & $2 k_{\min } a$ & $2 k_{*} a$ \\
\hline \multirow{4}{*}{0.1} & 1.0 & 50 & 0.098 & 0 \\
& 0.9 & 48 & 0.098 & 0.182 \\
& 0.8 & 49 & 0.098 & 0.266 \\
& 0.7 & 49 & 0.098 & 0.333 \\
& 1.0 & 50 & 0.124 & 0 \\
0.2 & 0.9 & 50 & 0.124 & 0.292 \\
& 0.8 & 50 & 0.124 & 0.439 \\
& 0.7 & 50 & 0.124 & 0.563 \\
& 1.0 & 50 & 0.142 & 0 \\
0.3 & 0.9 & 46 & 0.142 & 0.331 \\
& 0.8 & 47 & 0.142 & 0.510 \\
& 0.7 & 46 & 0.142 & 0.671 \\
& 1.0 & 100 & 0.156 & 0 \\
0.4 & 0.9 & 93 & 0.156 & 0.323 \\
& 0.8 & 94 & 0.156 & 0.511 \\
& 0.7 & 93 & 0.156 & 0.691 \\
\hline
\end{tabular}

momentum. To destroy the structure of the regular tiling, the system was evolved elastically $(\epsilon=1)$ for $\sim 80$ collisions per particle. For reference, the structure factors were also measured for these elastic systems. The driving and dissipation were switched on and the system was given time to relax to the stationary state for $\sim 1600$ collisions per particle.

For every combination of volume fraction $\varphi$, coefficient of restitution $\epsilon$ and driving mechanism, either $\sim 50$ or $\sim 100$ different initial conditions were simulated. All the measurements were averaged over the initial conditions. To check whether the stationary state had indeed been reached, 10 systems were evolved for an additional $\sim 800$ collisions per particle. No significant further evolution of the measured quantities was observed. Table 3.2 summarizes the simulation parameters.

\subsubsection{Measuring Correlation Functions}

To measure the static structure factor, $S(q)$, I first calculated

$$
\tilde{\rho}(\boldsymbol{k})=\frac{1}{N} \sum_{i} e^{i \boldsymbol{k} \cdot \boldsymbol{r}_{i}}
$$


for all $\boldsymbol{k}=\left(n_{x}, n_{y}, n_{z}\right) \Delta k, n_{\alpha}=1,2, \ldots$ where $\Delta k=4 \pi / L$ is the reciprocal lattice spacing, with the constraint that $|\boldsymbol{k}| \leq k_{\max }$. From this I got $\tilde{S}(\boldsymbol{k})=N \rho(\boldsymbol{k}) \rho^{*}(\boldsymbol{k})$.

Assuming a isotropic system, the structure factor, $\tilde{S}(\boldsymbol{k})$, must be independent of the direction $\hat{\boldsymbol{k}}$, i.e., $\tilde{S}(\boldsymbol{k}) \equiv S(k)$. This can be exploited to average $\tilde{S}(\boldsymbol{q})$ over all wave vectors with the same absolute value $k$. For larger wave numbers I additionally averaged over bins $[k, k+\delta k]$ of size $\delta k=\Delta k / 2$. To properly resolve the small $k$ divergence, I did not use the binning for small wave numbers. The averaging procedure allows to define a standard deviation, $\delta S(k)$, to quantify the precision of the results. To further reduce the standard deviation, the structure factors were finally averaged over different initial conditions. The error bars will be given as $\pm \delta S(q)$.

For the current correlators, the x-component of the total current,

$$
\tilde{\jmath}_{x}(k)=\frac{\sqrt{3}}{N} \sum_{i} \boldsymbol{v}_{i}^{x} \exp \left(i \boldsymbol{k} \cdot \boldsymbol{r}_{i}\right)
$$

and the longitudinal current,

$$
\tilde{\jmath}_{\ell}(k)=\frac{1}{N} \sum_{i}\left(\hat{\boldsymbol{k}} \cdot \boldsymbol{v}_{i}\right) \exp \left(i \boldsymbol{k} \cdot \boldsymbol{r}_{i}\right),
$$

were determined and then correlated in the same way as the density above to form $S_{\ell \ell}(k)$ and $S_{x x}(k)$. The transverse current correlator was then determined as $2 S_{\perp \perp}(k)=S_{x x}(k)-S_{\ell \ell}(k)$.

The temperature, $T(\boldsymbol{r})=m\left(\left\langle\boldsymbol{v}^{2}\right\rangle(\boldsymbol{r})-\boldsymbol{u}^{2}(\boldsymbol{r})\right) / 3$, and the flow velocity, $\boldsymbol{u}(\boldsymbol{r})$, can only be determined as local averages. The size of the averaging region shall be characterized by a length scale $\sigma$. To proceed, I determine $M=N \ell_{m}^{3} / \sigma^{3}$, uniformly distributed, random positions $\boldsymbol{R}_{\alpha}$. At each position, $\boldsymbol{R}_{\alpha}$, I determine the local flow velocity,

$$
\boldsymbol{u}_{\alpha} \equiv \boldsymbol{u}\left(\boldsymbol{R}_{\alpha}\right)=\sum_{i=1}^{N} w_{\sigma}\left(\boldsymbol{R}_{\alpha}-\boldsymbol{r}_{i}\right) \boldsymbol{v}_{i}
$$

and the local temperature,

$$
T_{\alpha} \equiv T\left(\boldsymbol{R}_{\alpha}\right)=\frac{m}{3}\left[\sum_{i}^{N} w_{\sigma}\left(\boldsymbol{R}_{\alpha}-\boldsymbol{r}_{i}\right) \boldsymbol{v}_{i}^{2}-\boldsymbol{u}_{\alpha}^{2}\right] .
$$

For the weighting function, I choose a gaussian, $w_{\sigma}(r) \propto \exp \left(-r^{2} / 2 \sigma^{2}\right)$. For the calculation of $S_{n T}(k)$, the density, $n\left(\boldsymbol{R}_{\alpha}\right)=\sum_{i} w\left(\boldsymbol{R}_{\alpha}-\boldsymbol{r}_{i}\right)$ is calculated analogously.

\subsection{Results}

The quantity that is measured most easily is the granular kinetic temperature, $T_{0}$, in the stationary state. Table 3.3 collects the measured temperatures relative to 
Table 3.3: Stationary temperature, $T_{0} / T_{0 E}$, relative to the Enskog prediction, $T_{0 E}$, as function of volume fraction, $\varphi$, and coefficient of restitution, $\epsilon$. The uncertainty is on the order of $2 \times 10^{-4}$. For every value of $\epsilon$, the first row concerns the naive volume driving while the second row is related to systems with local momentum conservation.

\begin{tabular}{ccccc}
\hline \multirow{2}{*}{ Restitution $\epsilon$} & \multicolumn{4}{c}{ Volume Fraction $\varphi$} \\
\cline { 2 - 5 } & 0.1 & 0.2 & 0.3 & 0.4 \\
\hline \multirow{2}{*}{0.9} & 1.0107 & 1.0197 & 1.0223 & 1.0154 \\
& 0.9949 & 0.9939 & 0.9916 & 0.9872 \\
0.8 & 1.0217 & 1.0419 & 1.0513 & 1.0412 \\
& 0.9896 & 0.9901 & 0.9884 & 0.9808 \\
0.7 & 1.0323 & 1.0668 & 1.0859 & 1.0745 \\
& 0.9857 & 0.9890 & 0.9887 & 0.9794 \\
\hline
\end{tabular}

the one expected from the driving parameters and the Enskog approximation, $T_{0 E}$. Especially the systems without momentum conservation show surprisingly large deviations from the Enskog prediction. Van Noije et al. [74] propose an approximate theory to explain these deviations as a coupling of the temperature modes to the other hydrodynamic modes. The deviations are significantly smaller if momentum conservation is restored but interestingly, the temperatures are now consistently lower than the prediction. The apparent maximum of $T_{0}$ for the naive volume driving in the $\varphi=0.3-0.4$ range should be studied more closely in the future.

\subsubsection{Static Correlation Functions}

Let me start with the static structure factor, $S(k)$, itself. Figs. 3.1 and 3.2 show the structure factor as I measured it from the simulations. For all parameters, the qualitative difference for small $k$ with regard to momentum conservation is easily visible. In agreement with Ref. [121] I find it hardly possible to extract the exponent from a fit to $k^{-\alpha}$ but the measurements are certainly compatible with $\alpha=2$ for the naive volume driving.

The height of the first peak is affected by both the degree of inelasticity and by the specific driving mechanism. A more detailed analysis of the detailed behavior of the structure factor at intermediate and large wave numbers $k$ must be relegated to future work. For the time being, I will only use the observation that the PY-approximation is not systematically worse for the inelastic fluid with locally momentum conserving driving as compared to the elastic case. For reference, I also show the direct correlation function, $n c(k)=1-S^{-1}(k)$, in Figs. 3.3 and 3.4 . Note that in case of naive volume 

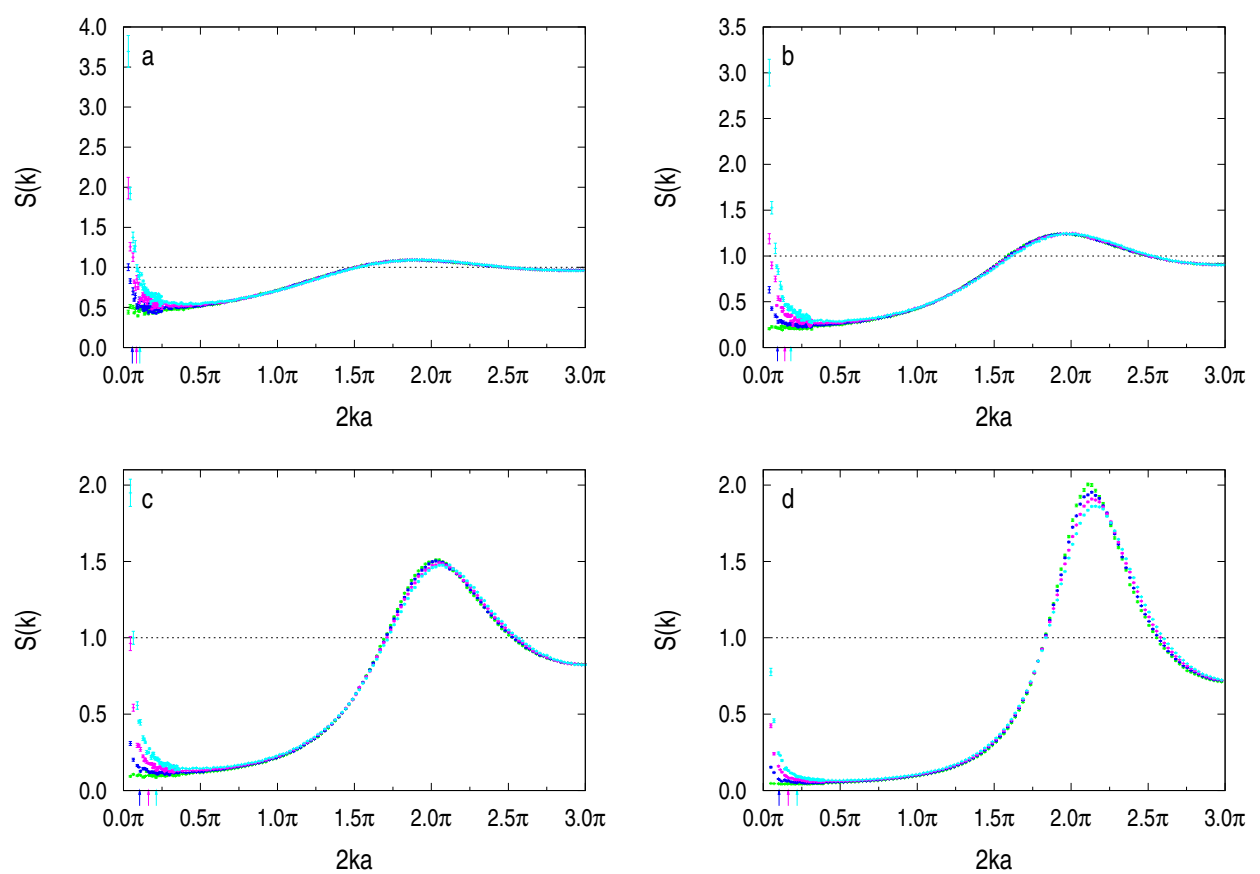

Figure 3.1: Static structure factor, $S(k)$, as a function of wavenumber, $k$, for an elastic hard sphere fluid (green) and for a granular fluid with naive volume driving and coefficient of restitution $\epsilon=0.9$ (dark blue), 0.8 (magenta), and 0.7 (light blue). The volume fraction is $\varphi=0.1,0.2,0.3,0.4$ from a-d. The arrows indicate the critical wavenumber, $k_{*}$ [see eq. (3.12)]

driving, the divergence of the structure factor, $S(k \rightarrow 0) \rightarrow \infty$, corresponds to $n c(k \rightarrow 0)=1$ for all densities and all values of the coefficient of restitution $\epsilon<1$.

For the longitudinal current correlator, $S_{\ell \ell}(k)$, (see Fig. 3.5) and even more so for the transverse current correlator, $S_{\perp \perp}(k)$, (see Fig. 3.7) the small $k$ divergence is quite prominent even for the finite system size considered here. No significant structure seems to be present for intermediate wave numbers.

For the momentum conserving driving, the longitudinal current correlator, $S_{\ell \ell}(k)$, (Fig. 3.6) shows a significantly smoother structure on large length scales compared to the equilibrium fluid. Remember that this is despite the possibly large internal random forces. The rise of $S_{\ell \ell}(k)$ for small wave numbers is not covered by the hydrodynamic theory discussed above which only predicts the limiting value, $S_{\ell \ell}(k \rightarrow 0)$. The transverse current correlator, $S_{\perp \perp}(k)$, (Fig. 3.8) on the other hand seems to be completely wave number independent. Different from the elastic case, there is a slight but significant non-equipartition, $S_{\perp \perp}(k)>S_{\ell \ell}(k)$, between the longitudinal and transverse modes. 

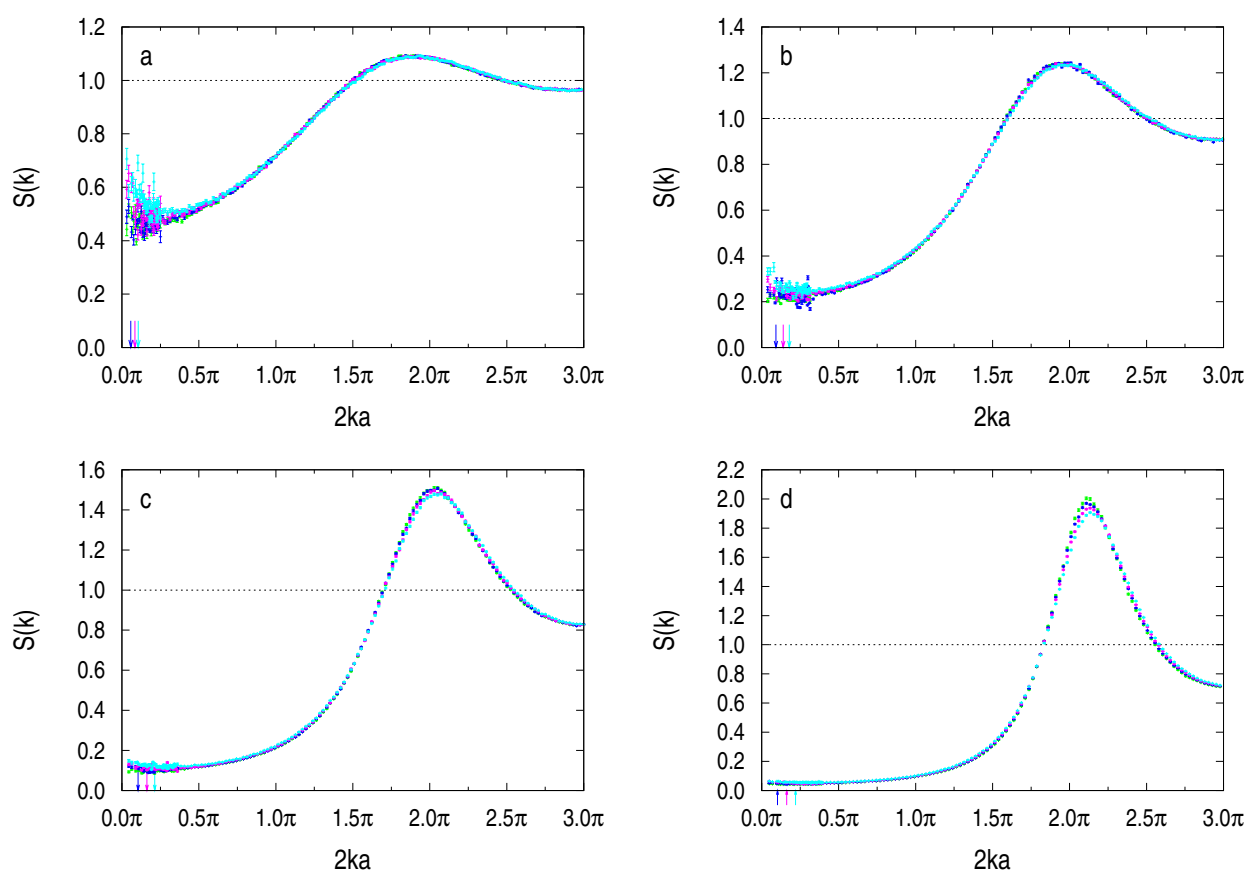

Figure 3.2: Static structure factor, $S(k)$, as a function of wavenumber $k$ for momentum conserving driving. See also Fig. 3.1

For the naive volume driving the density-temperature correlations, $S_{n T}(k)$, (see Fig. 3.9 a) are negative as expected. For the elastic hard sphere fluid, these correlations are expected to be absent. In Fig. 3.9p it can bee seen that the measurements show significant correlations on large length scales for the momentum conserving driving. While I do not understand this result completely, I believe that it is an artifact of the choice of weighting function, $w(r)$, used for the definition of the local temperature, $T(\boldsymbol{r})$. For the time being, the negative correlations in Fig. 3.9 should be considered with some caution. For the temperature correlator, $S_{T T}(k)$, (Fig. 3.10 the situation is even less clear.

\subsubsection{Speed of Sound}

The speed of sound, $c_{<}$, could be extracted for both types of driving. Table 3.4 summarizes the results. While the factor 5 between the squared speed of sound of an elastic fluid and an dissipative fluid expected in the low density limit is not achieved for the intermediate densities, sound waves are still considerably slowed down by the dissipative interactions. Moreover, there seems to be a small dependence of the speed of sound on the coefficient of restitution $\epsilon$, not captured by the hydrodynamic theory. 

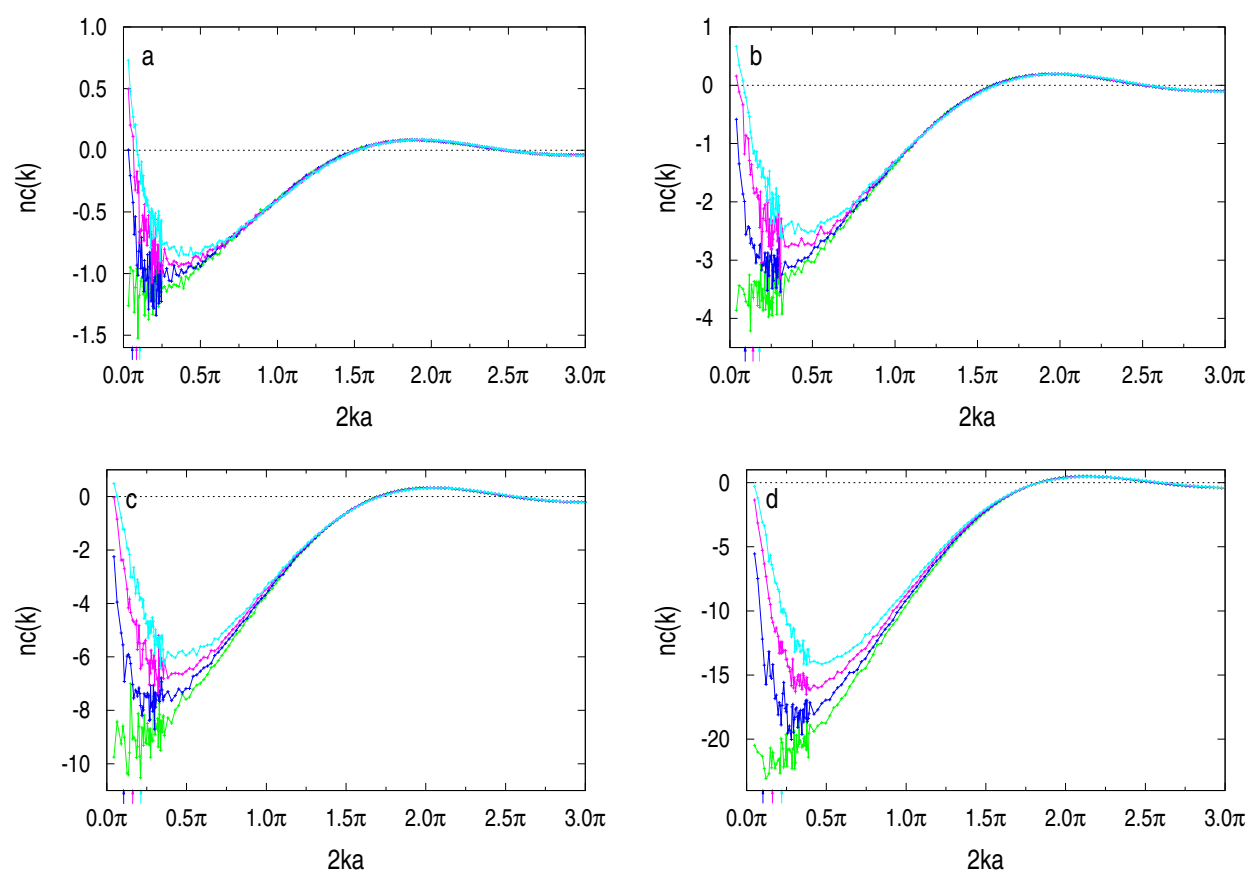

Figure 3.3: Direct Correlation Function, $n c(k)$, as a function of wave number, $k$, for naive volume driving. See also Fig. 3.1

One could also speculate that the speed of sound is slightly lower if the momentum conserving driving is used, but a statistically significant conclusion cannot be drawn from the current data.

\subsubsection{Shear Viscosity and Sound Damping for Naive Volume Driving}

From the transverse current correlator, $S_{\perp \perp}(k)$, alone, one can extract both the shear viscosity, $\eta$, as well as the ratio $\eta^{\text {int }} / \eta$, quantifying the deviations from the equilibrium FDT. The latter quantity is summarized in table 3.5. One observes an increasing deviation from unity both for increasing dissipation and increasing density. All values being less than one means that the fluctuations induced by the microscopic degrees of freedom are suppressed in an granular fluid as compared to an elastic fluid in thermal equilibrium. The result that $\eta^{\text {int }}$ only slightly deviates from $\eta$ supports the conclusion of Ref. 222 that $\eta^{\text {int }} \equiv \eta$ is a good approximation.

The theoretical prediction, eq. 3.34, can be written in the form

$$
\frac{m}{T_{0}} S_{\perp \perp}(k) \simeq S_{-2}^{\perp \perp} \cdot(k d)^{-2}+\eta^{\mathrm{int}} / \eta
$$


Table 3.4: Speed of sound, $m c_{<}^{2} / T_{0}$, in units of the thermal velocity, $\sqrt{T_{0} / m}$, as a function of volume fraction, $\varphi$, and coefficient of restitution, $\epsilon$. For the inelastic systems, the first row concerns the naive volume driving while the second row contains data from the momentum conserving driving. The uncertainty is given as one standard deviation.

\begin{tabular}{clccc}
\hline \multirow{2}{*}{ Restitution $\epsilon$} & \multicolumn{4}{c}{ Volume Fraction $\varphi$} \\
\cline { 2 - 5 } & \multicolumn{1}{c}{0.1} & 0.2 & 0.3 & 0.4 \\
\hline \multirow{2}{*}{1.0} & $2.18(2)$ & $4.61(5)$ & $10.4(1)$ & $23.7(3)$ \\
& $0.9(3)$ & $2.5(5)$ & $6 .(1)$ & $11 .(2)$ \\
0.9 & $1.0(2)$ & $2.0(4)$ & $3.9(7)$ & $11 .(1)$ \\
& $1.1(2)$ & $2.7(2)$ & $4.5(8)$ & $10.6(9)$ \\
0.8 & $0.8(1)$ & $1.9(2)$ & $4.4(4)$ & $9.1(8)$ \\
& $1.0(2)$ & $2.2(2)$ & $4.1(4)$ & $8.9(7)$ \\
0.7 & $0.78(8)$ & $1.8(1)$ & $3.9(4)$ & $9.1(5)$ \\
\hline
\end{tabular}

Table 3.5: Ratio $\eta^{\text {int }} / \eta$ that quantifies the deviation from the equilibrium FDT for the naive volume driving.

\begin{tabular}{ccccc}
\hline \multirow{2}{*}{ Restitution $\epsilon$} & \multicolumn{4}{c}{ Volume Fraction $\varphi$} \\
\cline { 2 - 5 } & 0.1 & 0.2 & 0.3 & 0.4 \\
\hline 0.9 & $0.992(2)$ & $0.978(3)$ & $0.962(3)$ & $0.967(3)$ \\
0.8 & $0.988(2)$ & $0.952(3)$ & $0.929(4)$ & $0.929(3)$ \\
0.7 & $0.981(3)$ & $0.928(4)$ & $0.890(5)$ & $0.885(4)$ \\
\hline
\end{tabular}



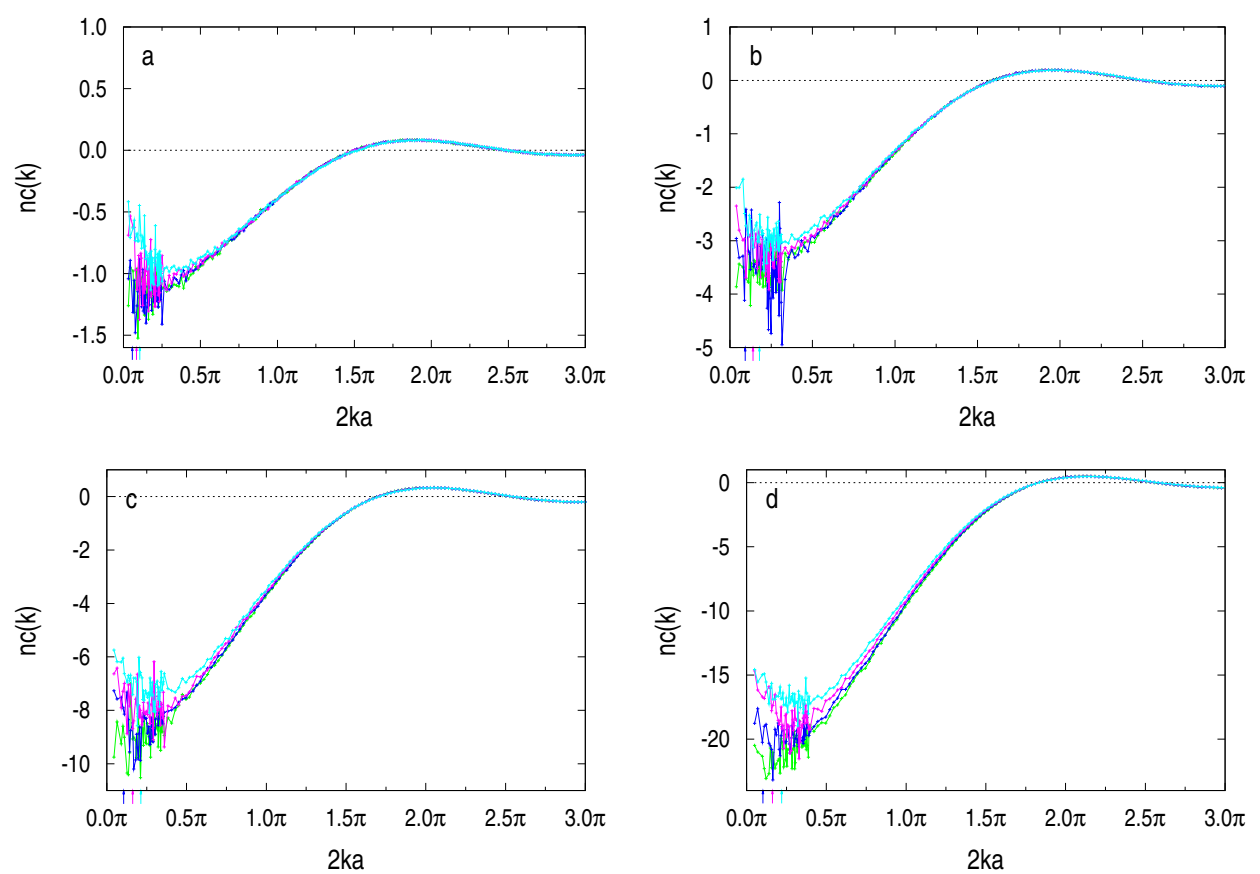

Figure 3.4: Direct Correlation Function, $n c(k)$, as a function of wave number, $k$, for momentum conserving driving. See also Fig. 3.1 .

where, $S_{-2}^{\perp \perp}=36 \gamma \varphi^{2} \chi^{2} / \nu_{\perp}^{*}$, and $\nu_{\perp}^{*}=\nu_{\perp} / \omega_{0} \ell_{0}^{2}$ is the dimensionless shear viscosity. In principle, different approximations could be used for $\gamma$ and $\chi$. Therefore, I report both $\nu_{\perp}^{*}$ as well as $\nu_{\perp}^{*} / \gamma \chi^{2}$ in table 3.6 . Like in an elastic fluid, the shear viscosity rises with density and is expected to diverge at the freezing density, $\varphi_{f}$, or, alternatively, at the glass transition density, $\varphi_{g}$. The observation that the viscosity is a decreasing function of the coefficient of restitution will be a recurring theme in the following chapters.

Garzó and Montanero [219] have determined the transport coefficients of a driven granular gas via the Chapman-Enskog method. For reference, I give their expression in units of $\omega_{0} \ell_{0}^{2}$ :

$$
\nu_{\perp}^{\mathrm{CE}}=\frac{5 \pi}{8} \chi \frac{1+\frac{2}{5} \varphi \chi(1+\epsilon)(3 \epsilon-1)}{(3-\epsilon)(1+\epsilon)}\left[1+\frac{4}{5} \varphi \chi(1+\epsilon)\right]+\frac{12}{5} \varphi^{2} \chi^{2}(1+\epsilon) .
$$

The agreement between simulation and theory is rather good, although the ChapmanEnskog method seems to systematically underestimate the value of the shear viscosity.

An expression completely analogous to eq. (3.47) holds for the sound damping constant $D_{S}^{*}=D_{S} / \omega_{0} \ell_{0}^{2}$. Sound damping is less strongly dependent on density 

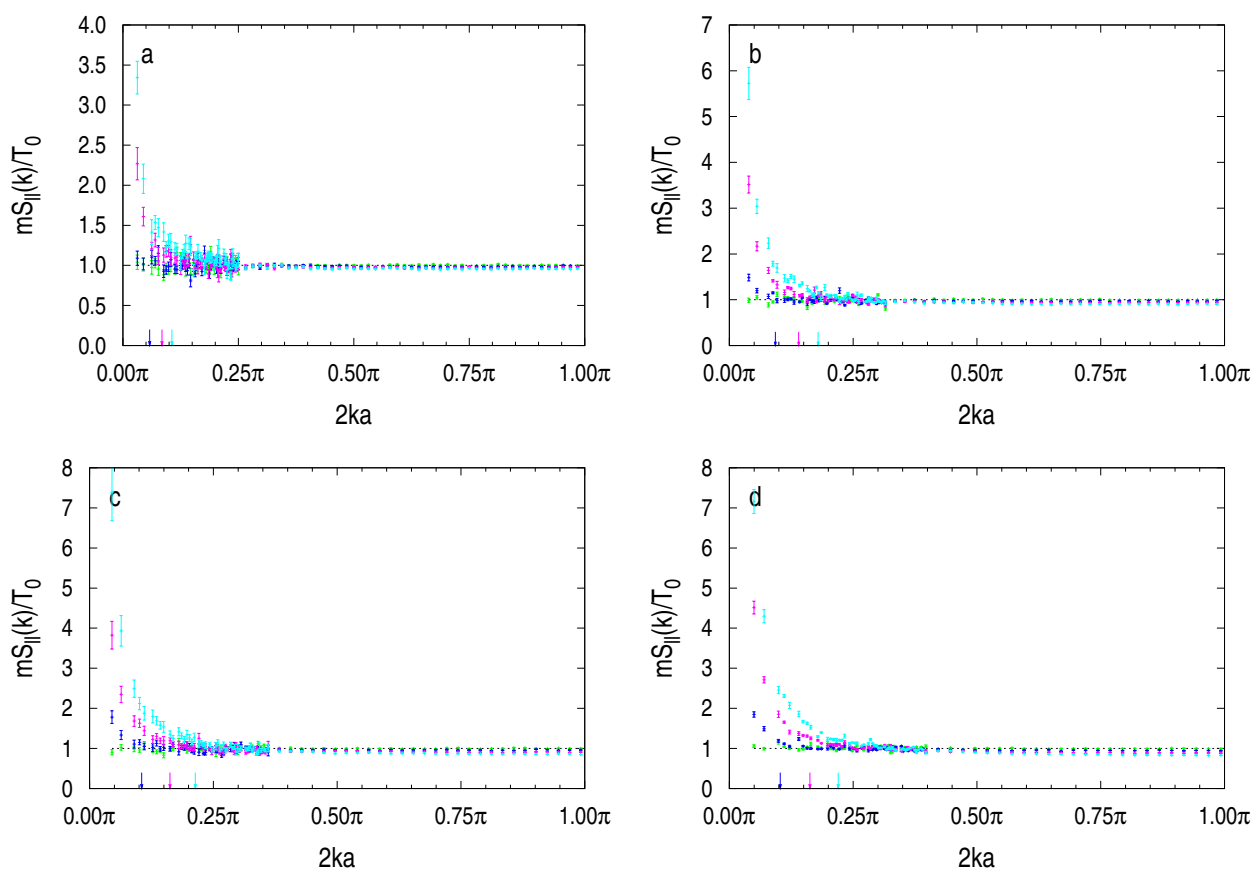

Figure 3.5: Longitudinal current correlator, $S_{\ell \ell}(k)$, as a function of wave number, $k$, relative to its uncorrelated value $T_{0} / m$ for naive volume driving. See also Fig. 3.1

but the dependence on the coefficient of restitution, $\epsilon$, is rather strong. This is in agreement with the prediction from fluctuating hydrodynamics, eq. (3.17), which indicates a divergence of $D_{S} \propto\left(1-\epsilon^{2}\right)^{-1}$.

As I have no information about the value of kinematic viscosity, $\nu_{\ell}$, I can only compare the measured sound damping, $D_{S}^{*}$, to the transport coefficient $D_{\Gamma}$, peculiar to a granular system. Assuming, that the values of the sound damping constant, $D_{S}^{<}$, from the hydrodynamic theory are reasonably correct, one expects that the kinematic viscosity, $\nu_{\ell}$, is roughly proportional to $D_{\Gamma}$.

\subsection{Discussion}

In this chapter, I explored the static structure of a driven granular fluid in a stationary state. The simulations described in section 3.2 and the measurements reported in Figs. 3.13 .10 are an attempt to cover a broad range of fluid densities, $\varphi=0.1-0.4$, as well as different degrees of moderate inelasticities, quantified by a coefficient of restitution $\epsilon \geq 0.7$.

Using the fluctuating hydrodynamic theory discussed in section 3.1, I could show explicitly that the small wave number divergence of the static correlation functions, 

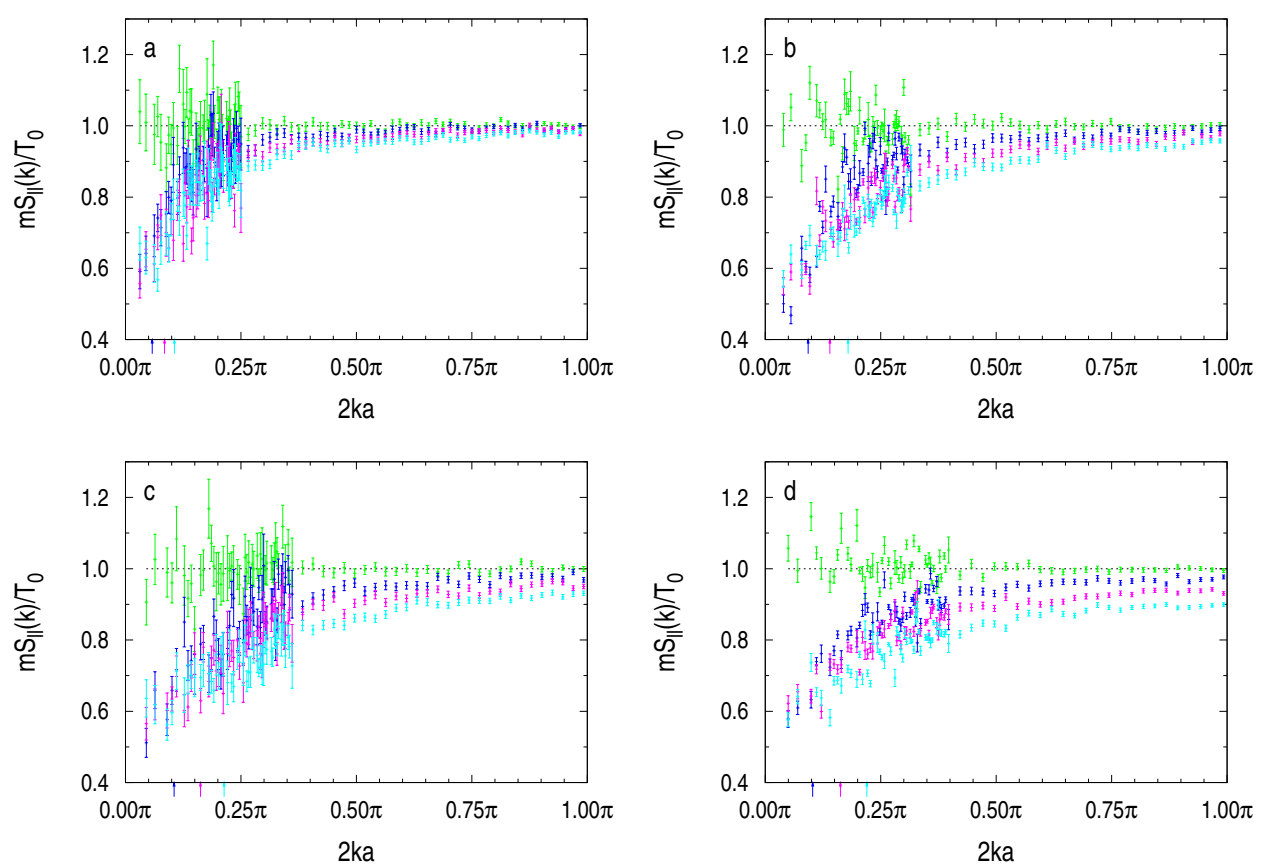

Figure 3.6: Longitudinal current correlator, $S_{\ell \ell}(k)$, as a function of wave number, $k$, relative to its uncorrelated value $T_{0} / m$ for momentum conserving driving. See also Fig. 3.1

$S_{a a}(k \rightarrow 0) \propto k^{-2}$, is a result of the combination of momentum conserving interactions of the granular particles and the naive volume driving that does not respect momentum conservation.

For a fluid in thermal equilibrium, the longitudinal current correlator, $S_{\ell \ell}(k)=$ $T / m$, is independent of the wave number $k$. My simulations showed that this does not hold for the granular fluid, even for the momentum conserving driving. Nevertheless, the speed of sound, $c$, can be determined from the ratio $S_{\ell \ell}(k) / S(k)$. In agreement with the hydrodynamic theory, the speed of sound is reduced for inelastic hard spheres compared to the elastic case. The speed of sound measured from the static correlations here, are identical within error bars to the results from the dynamic correlation functions [235].

Experimentally, it is difficult to generate a homogeneous, three dimensional granular fluid. Consequently, experimental measurements of the speed of sound in such a system are lacking. For measurements in a quasi two dimensional system, see, e.g., Ref. [236].

For the naive volume driving, I could measure the shear viscosity, $\nu_{\perp}$, and the sound damping constant, $D_{S}^{<}$. The measurement of the shear viscosity validates the 

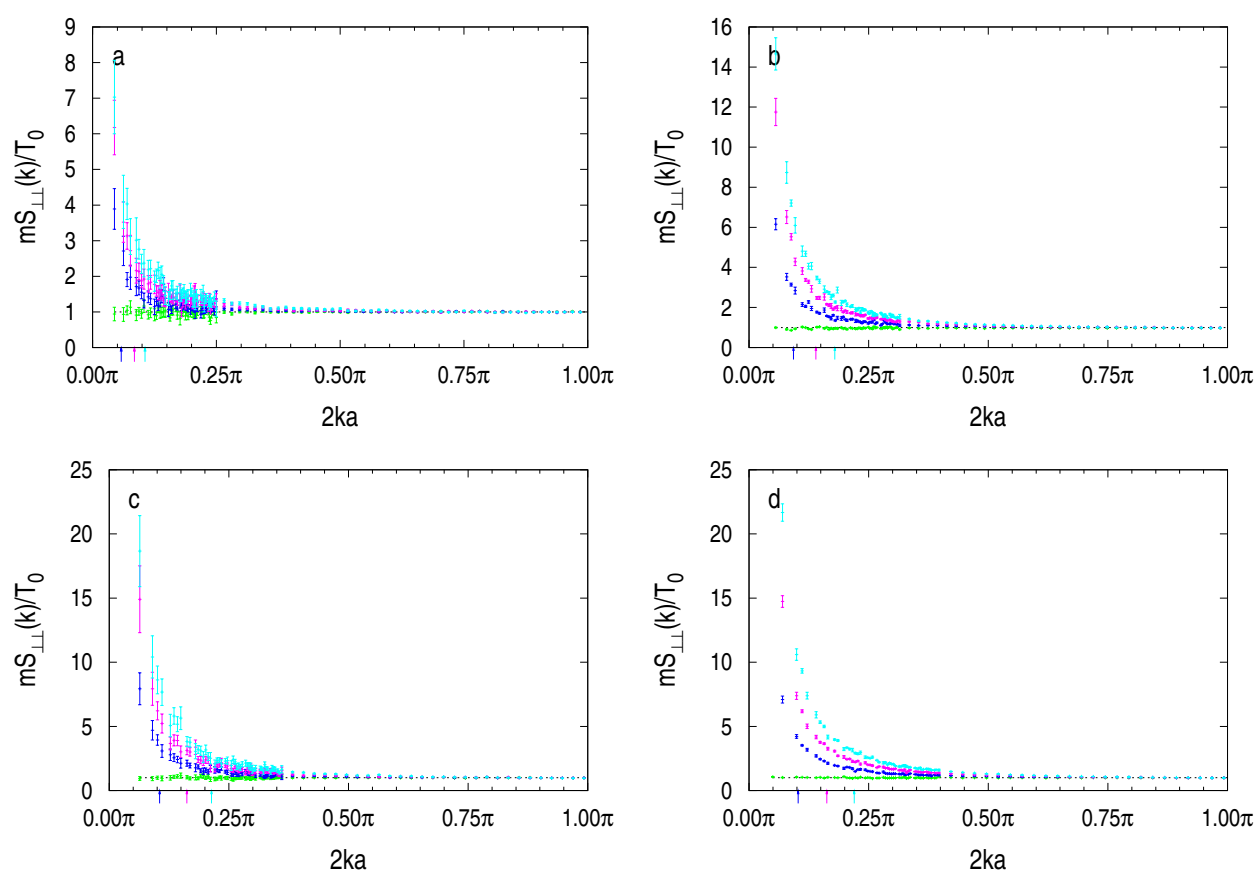

Figure 3.7: Transverse current correlator, $S_{\perp \perp}(k)$, as a function of wave number, $k$, relative to its uncorrelated value $T_{0} / m$ for naive volume driving. See also Fig. 3.1.

Chapman-Enskog expansion performed by Garzó and Montanero [219]. Independent of the density, the viscosity slightly decreases with increasing inelasticity. The surprising prediction that the sound damping diverges in the elastic limit is also supported by the simulation results. The fluctuations of the internal transverse random force, $\boldsymbol{\xi}_{\perp}$, were found to be slightly suppressed, $\eta^{\text {int }} \lesssim \eta$, compared to an fluid in thermal equilibrium. A similar ratio has been determined experimentally [237] and interpreted as an effective temperature $T_{\text {eff }}$.

For the momentum conserving driving, the transport coefficients could not be determined. Instead, the measurements allowed the proposition of two non-equilibrium FDTs,

$$
\begin{gathered}
\nu_{\perp}^{\text {int }}=\nu_{\perp}-\frac{\Gamma}{2 T} \ell^{2}, \\
\nu_{\ell}^{\text {int }}=\frac{1}{4} D_{S}^{<}-\frac{\Gamma}{2 T} \ell^{2} .
\end{gathered}
$$

For the theoretical analysis of the simulation results, I focused on the regime of large length scales. It would be desirable to have theoretical predictions for the 

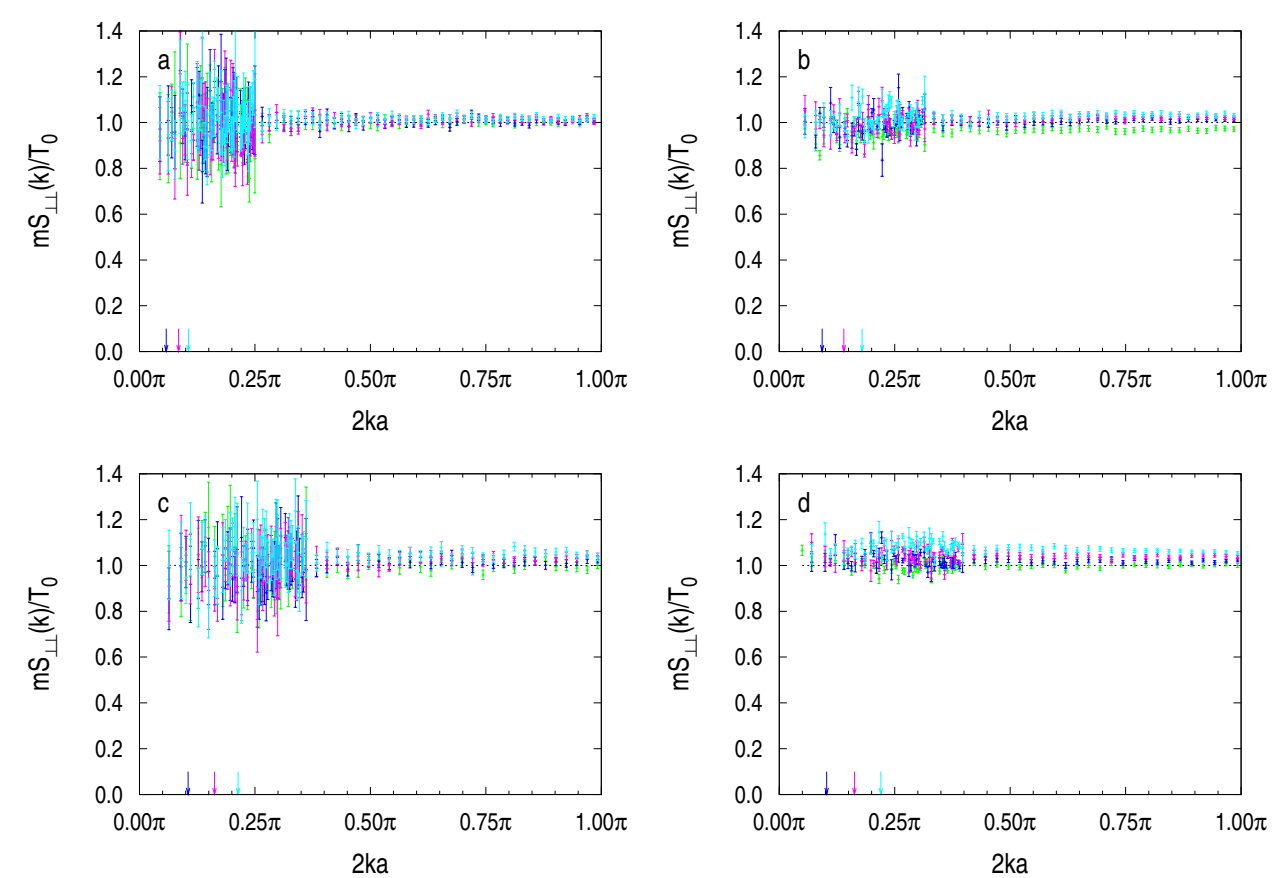

Figure 3.8: Transverse current correlator, $S_{\perp \perp}(k)$, as a function of wave number, $k$, relative to its uncorrelated value $T_{0} / m$ for momentum conserving driving. See also Fig. 3.1

intermediate and short range regime similar to, e.g., the Percus-Yevick equation for elastic hard spheres.

On a quantitative level, it would also be interesting to explore the effect of different collision rules. As long as the interactions are momentum conserving, the qualitative behavior regarding the small wave number divergence of the static correlation functions will remain unchanged, though. The study of rough spheres would also be interesting but likely quite challenging [238, 239]. Simulation results on the viscosity are available from Walton and Braun [240].

The second possibility to suppress the small $k$ divergence, namely an equation of motion that does not conserve momentum has been studied independently [241]. There, a viscous friction force, $-\gamma \boldsymbol{v}$, inhibits the propagation of large wave length sound modes.

Generalized Green-Kubo relations have recently been developed for granular fluids [242, 243. It would be interesting to see, which method allows for the most precise determination of the transport coefficients. Finally, if the problems with determining the temperature field indicated above could be resolved, one could also get information about the heat conductivity, $\kappa$, and the equation of state. 

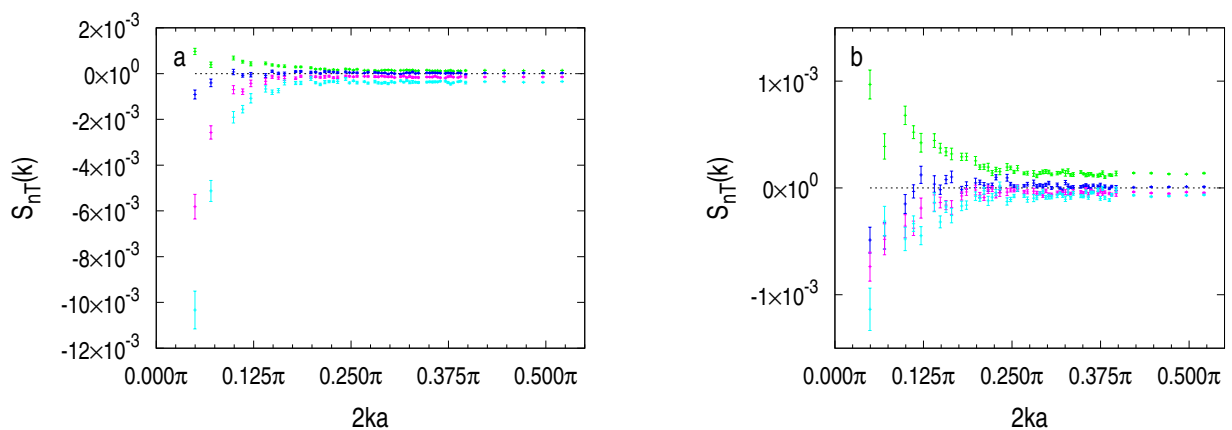

Figure 3.9: Density-Temperature cross-correlation function, $S_{n T}(k)$, as a function of wave number, $k$, for volume fraction $\varphi=0.4$ and a) naive volume driving and $\mathrm{b}$ ) momentum conserving driving. Colors are as in Fig. 3.1 Note the reduced range in $k$ compared to the previous figures.
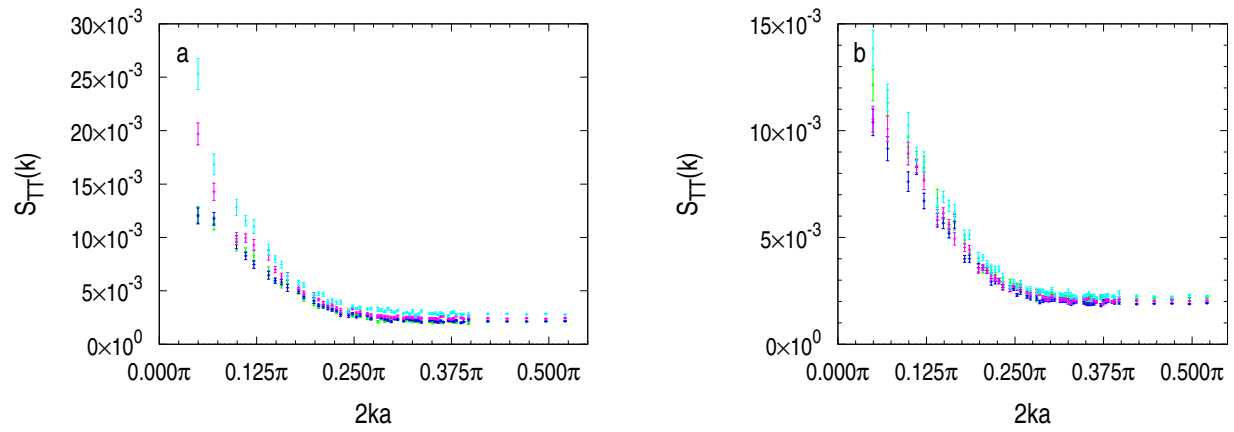

Figure 3.10: Temperature correlator, $S_{T T}(k)$, as a function of wavenumber, $k$, for volume fraction $\varphi=0.4$ and a) naive volume driving and b) momentum conserving driving. Colors are as in Fig. 3.1 Note the reduced range in $k$ compared to the previous figures. 
Table 3.6: Transport Coefficients. The dimensionless shear viscosity, $\eta_{\perp}^{*}$, and the dimensionless sound damping constant, $D_{S}^{*}$, as a function of volume fraction, $\varphi$, and coefficient of restitution, $\epsilon$. For comparison, the theoretical expressions $\nu_{\perp}^{\mathrm{CE}}$ [see, eq. (3.48)] and $D_{\Gamma}$ [see eq. (3.17)] are also listed.

\begin{tabular}{cccccllc}
\hline$\varphi$ & $\epsilon$ & $\nu_{\perp}^{*} / \gamma \chi^{2}$ & $\nu_{\perp}^{*}$ & $\nu_{\perp}^{\mathrm{CE}}$ & $D_{S}^{*} / 10^{2} \gamma \chi^{2}$ & $D_{S}^{*} / 10^{2}$ & $D_{\Gamma} / 10^{2}$ \\
\hline \multirow{4}{*}{0.1} & 0.9 & $13.0(6)$ & $1.70(8)$ & 1.12 & $3 .(1)$ & $0.4(2)$ & 0.064 \\
& 0.8 & $6.6(2)$ & $1.64(4)$ & 1.08 & $0.28(2)$ & $0.070(5)$ & 0.034 \\
& 0.7 & $4.3(1)$ & $1.52(4)$ & 1.04 & $0.138(5)$ & $0.049(2)$ & 0.024 \\
& 0.9 & $17.4(4)$ & $5.0(2)$ & 3.56 & $1.8(2)$ & $0.53(6)$ & 0.171 \\
0.2 & 0.8 & $8.52(8)$ & $4.72(4)$ & 3.27 & $0.38(2)$ & $0.21(1)$ & 0.090 \\
& 0.7 & $5.64(6)$ & $4.42(4)$ & 3.01 & $0.190(7)$ & $0.149(6)$ & 0.064 \\
& 0.9 & $23.6(6)$ & $16.8(4)$ & 14.2 & $2.0(3)$ & $1.5(2)$ & 0.51 \\
0.3 & 0.8 & $12.2(2)$ & $16.4(2)$ & 12.6 & $0.53(2)$ & $0.72(3)$ & 0.27 \\
& 0.7 & $8.1(2)$ & $15.4(4)$ & 11.1 & $0.239(7)$ & $0.46(1)$ & 0.19 \\
& 0.9 & $36.0(4)$ & $70.4(8)$ & 68.8 & $2.3(2)$ & $4.6(4)$ & 1.78 \\
0.4 & 0.8 & $17.6(1)$ & $65.4(4)$ & 58.8 & $0.61(2)$ & $2.24(7)$ & 0.94 \\
& 0.7 & $11.40(8)$ & $59.8(4)$ & 50.0 & $0.305(6)$ & $1.60(3)$ & 0.66 \\
\hline
\end{tabular}





\section{Mode Coupling Theories: An Overview}

Most derivations of mode coupling theories either explicitly or implicitly used projection operators [244] introduced by Zwanzig [245, 246] and Mori [247, 248]. This is also the approach I will use in the derivation of the granular mode coupling theories. While I found it well suited for a detailed calculation, the abundance of technical details can easily obscure the underlying idea. To get an intuitive understanding, I found a diagramatical representation helpful instead 1

In the next section I will introduce the formally exact equations of motion for the VACF, $\psi(t)$, the coherent scattering function, $\phi(q, t)$ and the incoherent scattering function, $\phi^{s}(q, t)$. In sections 4.24 .4 I will discuss the three types of mode coupling theory for the correlation functions $\psi(t), \phi(q, t)$ and $\phi^{s}(q, t)$. While, originally, both the equations of motion and the mode coupling approximations were derived for equilibrium fluids, in the subsequent chapters I will show explicitly that they also hold in the nonequilibrium stationary state of a randomly driven granular fluid.

\subsection{Memory Equations}

\subsubsection{The Velocity Autocorrelation Function}

The autonomous differential equation,

$$
\dot{\psi}_{0}(t)+\omega_{0} \psi_{0}(t)=0
$$

together with the initial condition $\psi_{0}(0)=1$ represents a particularly simple equation of motion for the velocity autocorrelation function, $\psi_{0}(t)=m\left\langle\boldsymbol{v}_{s}(0) \mid \boldsymbol{v}_{s}(t)\right\rangle / 3 T$, of a dilute gas. Intuitively, it describes the randomization of the initial velocity due to the scattering of the tagged particle off the particles of the surrounding fluid, $\dot{\boldsymbol{v}}(t)=-\omega_{0} \boldsymbol{v}(t)$. While it is nontrivial to deduce the dependence of the collision frequency, $\omega_{0}$, on the state of the system, this equation of motion necessarily describes an exponential decay of the velocity autocorrelation function. This is an exact result for short times [115], but the existence of the long time tails indicates that full

\footnotetext{
${ }^{1}$ See the publications by Andersen [249 251] and van Beijeren and Ernst [252] for much more thorough attempts to formulate kinetic theory in terms of diagrams.
} 
dynamics is more complicated. A formally exact equation of motion is given by the Langevin equation,

$$
\dot{\boldsymbol{v}}(t)+\omega_{0} \boldsymbol{v}(t)=\boldsymbol{f}(t),
$$

where the fluctuating force, $\boldsymbol{f}$, accounts for the influence of all the other degrees of freedom on the tagged particle's velocity [115]. It can be shown (see section 5 below) that the corresponding equation of motion for the $\mathrm{VACF}, \psi(t)$, reads

$$
\dot{\psi}(t)+\omega_{0} \psi(t)+\int_{0}^{t} M_{v}(t-\tau) \psi(\tau) d \tau=0
$$

where $M_{v}(t)$ is aptly called memory kernel [244, 115]. The solution of this full equation of motion can be written implicitly in terms of the solution, $\psi_{0}$, of the time local equation (4.1),

$$
\psi(t)=\psi_{0}(t)+\left(\psi_{0} \star M_{v} \star \psi\right)(t),
$$

where $\star$ denotes a convolution in time. A possible representation of this equation in terms of diagrams is given by

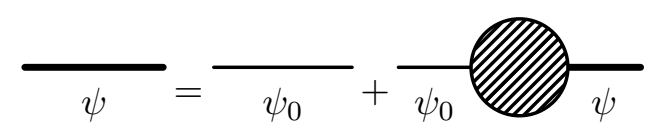

It has been found that the relaxation rate $\omega_{0}$ is smaller in a driven granular fluid by a factor $(1+\epsilon) / 2$ using an Enskog approximation [67].

\subsubsection{The Coherent Scattering Function}

Along the same lines, the hydrodynamic equations for an inviscid, isothermal fluid, the Euler equations, can be augmented by a fluctuating force. Consider the dynamic scattering function, $\phi(q, t)=N\left\langle\rho_{q}(0) \mid \rho_{q}(t)\right\rangle / S_{q}$, where, $\rho_{q}=\mathrm{FT}[\rho](q)=$ $\frac{1}{N} \sum_{j} \exp \left(i \boldsymbol{q} \cdot \boldsymbol{r}_{j}\right)$, is the Fourier transform of the density field, $\rho(\boldsymbol{r})=\frac{1}{N} \sum_{j} \delta\left(\boldsymbol{r}-\boldsymbol{r}_{j}\right)$, and the normalization is provided by the static structure factor, $S_{q}=N\left\langle\rho_{q}(t) \mid \rho_{q}(t)\right\rangle$. The corresponding equation of motion is given by,

$$
\ddot{\phi}(q, t)+\Omega_{q}^{2} \phi(q, t)+\int_{0}^{t} M(q, t-\tau) \dot{\phi}(q, \tau) d \tau=0 .
$$

The initial conditions are $\phi(q, 0)=1$ and $\dot{\phi}(q, 0)=0$ due to stationarity. The frequency $\Omega_{q}=q c(q)$ is essentially given by the generalized, wave number dependent speed of sound, $c(q)=\sqrt{T / m S_{q}}$ [115]. The formal solution of the above equation can again be written in the form of a Dyson equation,

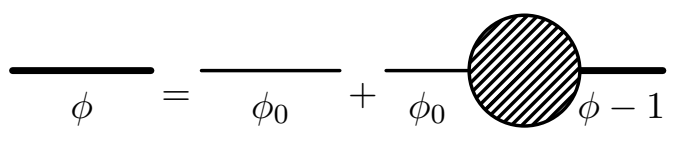


where $\phi_{0}(q, t)=\cos (c q t)$, the solution of eq. 4.6 without memory $[M(q, t) \equiv 0]$, describes undamped sound modes and $c=\lim _{q \rightarrow 0} c(q)$ is the long wave length speed of sound.

Either by Laplace transforming the above equation of motion or by looking at the Dyson equation (4.7), one finds that the EA order parameter, $f_{q}$, is given as a solution of the equation

$$
\frac{f_{q}}{1-f_{q}}=M(q, t \rightarrow \infty) / \Omega_{q}^{2}
$$

If the fluid is supposed to undergo a kinetic glass transition, signaled by $f_{q}>0$, then it also must have a nontrivial long time limit of the memory kernel $M(q, t \rightarrow \infty)>0$.

\section{New Results}

I am going to show in chapter 6 that the equation of motion 4.6 formally also holds for a driven granular fluid at least approximately. The speed of sound acquires a nontrivial dependence on the coefficient of restitution, $\epsilon$,

$$
c^{2}(q)=\frac{T}{m} \frac{1}{S_{q}}\left(\frac{1+\epsilon}{2}+\frac{1-\epsilon}{2} S_{q}\right)-\frac{(1+\epsilon)^{2}}{16} \omega_{E}^{2}\left[1-j_{0}(2 a q)+2 j_{2}(2 a q)\right]^{2} / q^{2} .
$$

\subsubsection{Tagged Particle Dynamics}

Motivating the equation of motion commonly employed for the incoherent scattering function, $\phi^{s}(q, t)$, is surprisingly nontrivial. A straightforward approach would yield a first order equation of motion, similar to the one for the VACF [eq. 4.3]]. Meaningful results are only obtained from a second order equation, formally identical to the one for the coherent scattering function, $\phi(q, t)$, [eq. [4.6)], though. Here, I can only give a sketch of a possible justification.

On macroscopic time scales, the inertial force on a tagged particle is small compared to the friction force, $-\gamma \boldsymbol{v}$, due to the presence of the other fluid particles.$^{2}$ In this case it is possible to formulate an equation of motion for the particle's spatial probability density, $\rho_{s}(\boldsymbol{r}, t)$, the Smoluchowski equation, independent of the particle's velocity [253.

The incoherent scattering function, $\phi^{s}(q, t)=\left\langle\rho_{q}^{s}(0) \mid \rho_{q}^{s}(t)\right\rangle$, of a fluid governed by the Smoluchowski equation can also be formulated in terms of a memory kernel, $\mathcal{M}^{s}(q, s)$. Later, it was found that consistency requires working in terms of a redefined,

\footnotetext{
${ }^{2}$ It is also possible to approximately describe the interaction of particles with the host fluid in a colloidal suspension in terms of a friction term $-\tilde{\gamma} \boldsymbol{v}$. Note that here I do not discuss a suspension but a pure fluid.
} 
irreducible memory kernel, $M_{s}^{\text {irr }}(q, t)$ [254, 198]. A relation between the two is given in the Laplace domain by

$$
\hat{M}_{s}^{\mathrm{irr}}(q, s)=-\frac{\hat{\mathcal{M}}_{s}(q, s)}{1+\tau_{q} \hat{\mathcal{M}}_{s}(q, s)},
$$

where $\tau_{q}:=1 / D_{0} q^{2}$ is the time scale of the diffusion process [255] and $D_{0}$ is the short time diffusion coefficient.

If one derives the equation of motion for the incoherent scattering function, $\phi^{s}(q, t)$, based on Newtonian dynamics, i.e., retaining inertial effects, it was found that on macroscopic time scales, the memory effects are essentially given by the same, irreducible memory kernel,

$$
\ddot{\phi} s(q, t)+\nu^{s} \dot{\phi}^{s}(q, t)+q^{2} \frac{T}{m} \phi^{s}(q, t)+\int_{0}^{t} M_{s}^{\mathrm{irr}}(q, t-\tau) \dot{\phi}^{s}(q, \tau) d \tau=0,
$$

where $\nu^{s}:=T / m D_{0}$ and the initial conditions are the same as in eq. (4.6) above: $\phi^{s}(q, 0)=1$ and $\dot{\phi}^{s}(q, 0)=0$ [255]. This explains the universality between the Brownian and Newtonian description in dense colloidal suspensions. Note that this equation of motion admits unphysical oscillatory solutions if one neglects the memory effects.

The asymptotic value ${ }^{3} f_{q}^{s}=\phi^{s}(q, t \rightarrow \infty)$, follows from the equation

$$
\frac{f_{q}^{s}}{1-f_{q}^{s}}=m M_{s}^{\mathrm{irr}}(q, t \rightarrow \infty) / q^{2} T
$$

which is the direct analogue of eq. (4.8) [258.

The mean square displacement, $\left\langle\delta r^{2}\right\rangle(t)$, can also be understood as the second order coefficient in a small wave number expansion of the incoherent scattering function, $\phi^{s}(q, t)=1-q^{2}\left\langle\delta r^{2}\right\rangle(t) / 6+\mathcal{O}\left(q^{4}\right)$. A simple but often successful approximation is to assume that the mean square displacement captures all of the time dependence and write the incoherent scattering function in the gaussian approximation, $\phi^{s}(q, t)=$ $\exp \left[-q^{2}\left\langle\delta r^{2}\right\rangle(t) / 6\right][115$, 235].

If one assumes that the dynamics in the fluid will eventually always be diffusive, $\lim _{t \rightarrow \infty}\left\langle\delta r^{2}\right\rangle(t)=6 D_{\infty} t$, one finds that the long time diffusion coefficient is determined by the time integral of the memory kernel $M_{0}(t):=\lim _{q \rightarrow 0} q^{2} M_{s}^{\text {irr }}(q, t)$ [257],

$$
D_{\infty}^{-1}=D_{0}^{-1}+\frac{m}{T} \int_{0}^{\infty} M_{0}(\tau) d \tau
$$

At the kinetic glass transition, the mean square displacement is bounded, $\left\langle\delta r^{2}\right\rangle(t \rightarrow$ $\infty)=r_{c}^{2}$, instead, and the localization length is (see Ref. [257]) given by

$$
r_{c}^{2}=3 T / m M_{0}(t \rightarrow \infty) .
$$

\footnotetext{
${ }^{3}$ Sometimes referred to as the Lamb-Mößbauer factor [256, 257
} 


\section{New Results}

In chapter 7 I will show that the equation of motion (4.11) also hold for a driven granular fluid. The relaxation rate $\nu^{s}$ is reduced by a factor $(1+\epsilon) / 2$ compared to a fluid of elastic hard spheres in agreement with the result for the VACF.

At this point, the equations of motion 4.3, 4.6 and 4.11 are exact but formal. If the memory kernels were known, the equations could be solved in the Laplace domain. Thus, knowing the exact memory kernel is equivalent to having a complete solution of the dynamical equations.

In the remaining sections of this chapter, I will briefly discuss the application of the mode coupling formalism to the three memory kernels introduced above. In particular, I will emphasize the differences between mode coupling theory as it has been applied to equilibrium systems and the nonequilibrium mode coupling as it is outlined in this thesis.

\subsection{Back Scattering and Long-Time-Tails}

The nontrivial behavior of the velocity autocorrelation function is a consequence of the interaction of the tagged particle with the surrounding fluid. On macroscopic time and length scales, one expects that the fluid is well described by hydrodynamic equations. The most basic way to approximately include the effect of the interaction between the tagged particle and the hydrodynamic modes is to retain the lowest order term in a perturbative expansion of the memory kernel (see also appendix D)

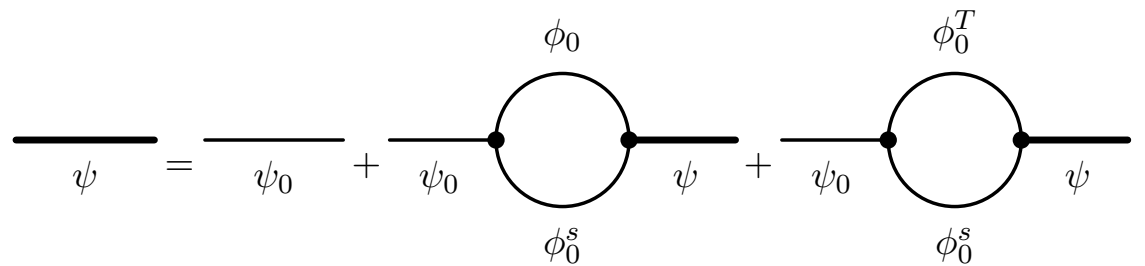

Detailed balance (see chapter 2 requires that the transition rates expressed by the left $\boldsymbol{\alpha}$ and the right vertex $\boldsymbol{\gamma}-$ are equal in equilibrium fluids.

As the suppression of diffusion and back scattering are attributed to the blocking effect of the dense host fluid, one should be able to get such an effect by explicitly treating the interaction of the tagged particle with the hydrodynamic density field, $\phi_{0}(q, t)=\exp \left(-D_{S} q^{2} t\right) \cos (c q t)$, characterized by the speed of sound, $c$, and the sound damping constant, $D_{S}$. The long time tails, on the other hand were found to be related to the transverse currents, $\boldsymbol{j}_{q}^{T}=\boldsymbol{j}_{q}-\hat{\boldsymbol{q}} j_{q}^{L}$. The corresponding hydrodynamic correlator, $\phi_{0}^{T}(q, t)=\exp \left(-\nu_{\perp} q^{2} t\right)$, is characterized by the shear viscosity, $\nu_{\perp}=\eta / m n$. Indeed, 
one finds (see chapter 5) that the memory kernel is given asymptotically as

$$
M_{v}(t) \propto \int_{0}^{\infty} q^{2} e^{-(D+\eta) q^{2} t} d q \propto[(D+\eta) t]^{-3 / 2}
$$

reproducing the long time tail result.

Inspired by the original explanation of the long time tails by Ernst et al. [259], there are quite a few derivations of mode coupling approximations for the velocity autocorrelation function [260-268]. Starting from either kinetic theory or fluctuating hydrodynamics, they mainly differ in two points: (i) The approximations used in determining the vertices and (ii) the precise form of the correlation functions that are used for the loops. While the theory as it is presented above can essentially only explain the asymptotic regime, $t \rightarrow \infty$, some groups tried to formulate mode coupling theories that yield reasonable results for all times. This can be done by replacing the hydrodynamic correlators by correlation functions with a larger range of validity and it allows, e.g., to calculate the coefficient of diffusion self consistently. For particles with a smooth potential, mode coupling has to be applied to the second order memory function, see, e.g., Ref. [269].

Apart form the mode coupling approach, the velocity autocorrelation function has also been calculated with the help of other kinetic theories, see e.g., Refs. [270 273].

\section{New Results}

In chapter 5 . I will derive the mode coupling expression for the memory kernel of a driven granular fluid via the projection operator formalism. The resulting equations are formally equivalent to those in the elastic case. In particular, the result for the long time tails, $\psi(t) \propto t^{-3 / 2}$, will be recovered. What is fundamentally different is that the granular fluid violates detailed balance and consequently the left and right vertex are different. More specifically, the ability of the tagged particle to excite coherent density waves is reduced as compared to the elastic case. In turn, this reduces the efficiency of the back scattering, which results in an increased diffusivity of the granular fluid.

\subsection{The Coherent Scattering Function and the Glass Transition}

While the mode coupling theory for the velocity autocorrelation could be interpreted as the inclusion of some first order terms of a perturbation series, no such straightforward interpretation is possible for the most widely used mode coupling theory for the coherent scattering function. First, the memory kernel, $M(q, t) \approx \nu_{q} \delta(t)+M_{\mathrm{MCT}}[\phi](q, t)$, is assumed to be composed of a quickly decaying 
part, approximated by a delta function in time and an additional part that shall be approximated in the spirit of mode coupling theory. In terms of diagrams, $M_{\mathrm{MCT}}[\phi]$ it is given as

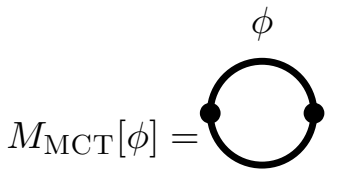

Note the heavy lines in the loop, denoting the full correlation function. This turns the equation of motion (4.6) into a recursive definition of the coherent scattering function, $\phi(q, t)$, in other words, the equation of motion has become a nonlinear integro-differential equation which has to be solved self-consistently.

Again, quite a number of authors have derived equations of this type for particles interacting via smooth potentials [274-277] and for hard spheres [278 280]. Götze and coworkers later based their extensive studies of mode coupling predictions on the derivation of the equations by Sjögren [281]. Boley [282] and Lindenfeld [283] were able to show that Mazenko's approach [274] is actually equivalent to the projection operator formalism. Here, and in a large part of the literature, the vertices are calculated with an Enskog approximation, i.e., spatial pair correlations are treated exactly while the velocities of different particles are assumed to be uncorrelated.

Applied to classical fluids, the mode coupling expression for the memory kernel constitutes an approximation but it is possible to construct physical models where the mode coupling memory kernel is exact [284]. On the other hand one can also find systems were it fails completely [285].

Bengtzelius et al. [258] realized that the nonlinear self-consistency equation admits bifurcations in the space of solutions. If the high temperature or low density solution with $f_{q}=0$ suddenly becomes unstable toward a solution with $f_{q} \neq 0$ this describes the kinetic glass transition. This behavior of the mode coupling equations becomes especially transparent for the schematic model [286]. Here, the additional complexity introduced via the wave number dependence of the scattering function is avoided by approximating the static scattering function by a delta function, $S_{q}=1+S^{*} \delta\left(q-q^{*}\right)$. The characteristic wave number $q^{*}$ is chosen as the location of the first and highest peak of $S_{q}$. The schematic mode coupling equations then read

$$
\ddot{\phi}(t)+\Omega^{2} \phi(t)+4 \Lambda \Omega^{2} \int_{0}^{t} \phi^{2}(\tau) \dot{\phi}(t-\tau) d \tau=0,
$$

where the strength of the memory effect is controlled by the coupling constant $\Lambda$.

Equation (4.8), determining the EA order parameter, $f$, turns into a simple algebraic equation, $f /(1-f)=4 \Lambda f^{2}$. For $\Lambda<1$, the only real solution is $f \equiv 0$, indicating fluid behavior while for $\Lambda \geq 1$, the only stable solution turns out to be $f \geq 1 / 2$, i.e., glassy behavior. A careful analysis (see, e.g., Ref. [287]) shows that the 
bifurcation scenario, where $f_{q}$ jumps from $f_{q} \equiv 0$ to $f_{q}>0$ discontinuously, carries over to the fluid model where everything depends on the wave number $q$.

From an expansion around the critical state at the glass transition, one can confirm the von Schweidler law,

$$
\phi(q, t)-f_{q} \propto-t^{b},
$$

and a similar power law behavior for the $\beta$-relaxation,

$$
\phi(q, t)-f_{q} \propto t^{-a},
$$

for a fluid close to the glass transition [287]. The critical exponents, $a$ and $b$, are related to a single critical parameter $\lambda$ via

$$
\lambda=\frac{\Gamma^{2}(1-a)}{\Gamma(1-2 a)}=\frac{\Gamma^{2}(1+b)}{\Gamma(1+2 b)}
$$

where $\Gamma(x)$ is the Gamma function.

As a result of the Enskog approximation used to calculate the vertices and the relaxation frequencies, the static structure factor, $S_{q}$, is a parameter of the theory. Choosing the Percus-Yevick approximation of $S_{q}$, one arrives at a critical density $\varphi_{c}^{P Y} \approx 0.516[258$. Using extrapolated structure factors from simulation measurements, the value is slightly higher, $\varphi_{c} \approx 0.546[288]$.

\section{New Results}

For a driven granular fluid, the vertices and the relaxation frequency, $\Omega_{q}^{2}$, deviate from their elastic values in such a way, that the memory kernel for the dissipative fluid, $m_{\mathrm{MCT}}^{\epsilon}[\phi](q, t)=A(q, \epsilon) m_{\mathrm{MCT}}[\phi](q, t)$, acquires a positive prefactor $A(q, \epsilon)$ that depends on the wave number $q$ and on the coefficient of restitution $\epsilon$. An important prerequisite for mode coupling theory to be mathematically sound is that the memory kernel is positive definite [287]. This property was shown for the memory kernel of the elastic hard sphere fluid and is manifestly retained for the driven granular fluid. Consequently, the granular mode coupling theory predicts a granular glass transition for all values of the coefficient of restitution. With increasing dissipation, the critical density, $\varphi_{c}(\epsilon)$, smoothly increases from the elastic value (see Fig. 1.9).

\subsection{Coupling of the Tagged Particle to the Coherent Modes}

To determine the coupling of a tagged particle to the collective density modes, a third kind of mode coupling theory can be used [289, 258, 290] 


$$
M_{\mathrm{MCT}}^{\mathrm{irr}}\left[\phi, \phi^{s}\right]=\bigodot_{\phi^{s}}^{\phi}
$$

For a mode coupling treatment of the tagged particle dynamics of soft particles see, e.g., Ref. [291].

Close to the glass transition, it is natural to choose $\phi(q, t) \equiv \phi_{\mathrm{MCT}}(q, t)$ [258], the solution of the mode coupling equation (4.6) for the coherent part of the loop. Again, this turns the equation of motion into a closed equation for $\phi^{s}(q, t)$ that has to be solved self consistently. Although it is widely assumed that this a successful description of the tagged particle dynamics, besides Refs. [289, 290], there is almost no discussion of the physical motivation for this approximation.

For the full time dependence the equation of motion can, again, only be solved numerically. The asymptotic value of the incoherent scattering function, $f_{q}^{s}$, follows the behavior of the EA order parameter, $f_{q}$, and becomes nonzero at the glass transition.

\section{New Results}

In chapter $7 \mathrm{I}$ will show how to carry out this type of mode coupling theory for the driven granular fluid. It turns out that the memory kernel, $m_{\mathrm{MCT}}^{\epsilon \text {,irr }}\left[\phi, \phi^{s}\right](q, t)=$ $(1+\epsilon) m_{\mathrm{MCT}}^{\mathrm{irr}}\left[\phi, \phi^{s}\right](q, t) / 2$, formally differs from the elastic expression only by a simple prefactor. The more inelastic the fluid, the weaker the memory effects and thus, the higher the diffusivity, $D_{\infty}$, for a given density. As the glass transition density is increased in the dissipative fluid for the same reason, the critical length scale, $r_{c}^{\epsilon}$, is nevertheless smaller than in the elastic fluid. 



\section{The Velocity Autocorrelation Function}

In this chapter I want to discuss the mode coupling description of the velocity autocorrelation function (VACF) of a driven granular fluid for densities far away from the glass transition. The main focus will be to find a description of the long time tail. I will first apply the projection operator formalism of mode coupling theory to an elastic hard sphere fluid in section 5.1. In the following section 5.2, I will discuss the complications introduced by the extension to a nonequilibrium dissipative fluid. With the feasibility of granular mode coupling established, I will present a granular mode coupling description of the long time tails in section 5.3 .

To find a mathematical description for the VACF, $\psi(t)$, that predicts a long time tail, $\psi(t \rightarrow \infty) \propto t^{-3 / 2}$, the memory kernel needs to have the same asymptotic behavior, $M_{v}(t \rightarrow \infty) \propto t^{-3 / 2}$. This can most easily be seen in the Laplace domain, where the VACF is given by, $\hat{\psi}(s)=\left[s-i \omega_{0}-\hat{M}_{v}(s)\right]^{-1}$ and where the long time tail corresponds to $\hat{\psi}(s \rightarrow 0) \propto \sqrt{s}$. An expansion of $\hat{\psi}(s)$ in powers of $\sqrt{s}$ only contains a linear term if $\hat{M}_{v}(s \rightarrow 0) \propto \sqrt{s}$ [115]. This implies that the memory kernel of the $\mathrm{VACF}$ of an elastic or inelastic hard sphere fluid must decay algebraically for long times, $M_{v}(t) \propto t^{-3 / 2}$.

\subsection{Mode Coupling Theory for Elastic Hard Spheres}

The results obtained in this section are a variant of those found in Refs. [261, 272]. Technically, I closely follow the formalism of Bosse et al. [269].

To get an explicit expression for the memory kernel of the VACF, $\psi(t)=$ $m\left\langle\boldsymbol{v}_{s} \mid \boldsymbol{v}_{s}(t)\right\rangle / 3 T$, I use the Mori identity (see appendix E) where the relevant projection operator is given by $\mathcal{P}=m\left|\boldsymbol{v}_{s}\right\rangle\left\langle\boldsymbol{v}_{s}\right| / 3 T$. Consequently, one gets for the VACF in the Laplace domain

$$
\hat{\psi}(s)=\left[s-i \omega_{0}-\hat{M}_{v}(s)\right]^{-1},
$$

where, $\omega_{0}=m\left\langle\boldsymbol{v}_{s} i \hat{\mathcal{L}}_{+} \boldsymbol{v}_{s}\right\rangle / 3 T$, is the collision frequency and the memory kernel is formally given by

$$
\hat{M}_{v}(s)=\frac{m}{3 T}\left\langle\boldsymbol{v}_{s} \mid \hat{\mathcal{L}}_{+} \mathcal{Q}\left(s-\mathcal{Q} \hat{\mathcal{L}}_{+} \mathcal{Q}\right)^{-1} \mathcal{Q} \hat{\mathcal{L}}_{+} \boldsymbol{v}_{s}\right\rangle
$$

and $\mathcal{Q}=1-\mathcal{P}$. This shows that the memory kernel, $M_{v}(t) \propto\langle\boldsymbol{F} \mid \boldsymbol{F}(t)\rangle$, can also be regarded as the autocorrelation of the so called fluctuating force, $\boldsymbol{F}=\mathcal{Q} \hat{\mathcal{L}}_{+} \boldsymbol{v}_{s}[244$. 
Let me introduce the mode coupling approximation with the coupling of the tagged particle to the collective density mode, $\rho_{k}=\frac{1}{N} \sum_{i} e^{i \boldsymbol{k} \cdot \boldsymbol{r}_{i}}$. Following the projection operator formulation of mode coupling theory, I introduce a second projection operator,

$$
\mathcal{P}_{\rho}=N \sum_{\boldsymbol{k}, \boldsymbol{p}}\left|\rho_{k} \rho_{p}^{s}\right\rangle\left\langle\rho_{k} \rho_{p}^{s}\right| / S_{k}
$$

where, $\rho_{p}^{s}=e^{i \boldsymbol{p} \cdot \boldsymbol{r}_{s}}$, is the probability density of the tagged particle and write

$$
M_{v}(t) \approx \frac{m}{3 T}\left\langle\boldsymbol{v}_{s} \mid \hat{\mathcal{L}}_{+} \mathcal{Q} \mathcal{P}_{\rho} \exp \left(i t \mathcal{Q} \hat{\mathcal{L}}_{+} \mathcal{Q}\right) \mathcal{P}_{\rho} \mathcal{Q} \hat{\mathcal{L}}_{+} \boldsymbol{v}_{s}\right\rangle .
$$

Using the definition of $\mathcal{P}_{\rho}$, one finds

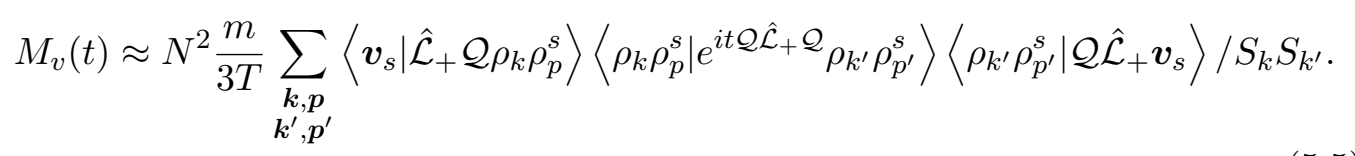

Two more approximations are needed to get a tractable expression for the memory kernel: A factorization approximation,

$$
\begin{aligned}
\left\langle\rho_{k} \rho_{p}^{s} \mid e^{i t \mathcal{Q} \hat{\mathcal{L}}_{+} \mathcal{Q}} \rho_{k^{\prime}} \rho_{p^{\prime}}^{s}\right\rangle & \approx\left\langle\rho_{k} \mid e^{i t \mathcal{Q} \hat{\mathcal{L}}_{+} \mathcal{Q}} \rho_{k^{\prime}}\right\rangle\left\langle\rho_{p}^{s} \mid e^{i t \mathcal{Q} \hat{\mathcal{L}}_{+} \mathcal{Q}} \rho_{p^{\prime}}^{s}\right\rangle \\
& =\left\langle\rho_{k} \mid e^{i t \mathcal{Q} \hat{\mathcal{L}}_{+} \mathcal{Q}} \rho_{k}\right\rangle\left\langle\rho_{p}^{s} \mid e^{i t \mathcal{Q} \hat{\mathcal{L}}_{+} \mathcal{Q}} \rho_{p}^{s}\right\rangle \delta_{k k^{\prime}} \delta_{\boldsymbol{p} p^{\prime}}
\end{aligned}
$$

where the second equality holds exactly for a homogeneous system (see, e.g., Ref. [287]). The second assumption is that for long times, higher powers of the reduced Liouville operator, $\tilde{\mathcal{L}}_{+}:=\mathcal{Q} \hat{\mathcal{L}}_{+} \mathcal{Q}$, become identical to those of the original operator, $\hat{\mathcal{L}}_{+}$, i.e., $\exp \left(i t \mathcal{Q} \hat{\mathcal{L}}_{+} \mathcal{Q}\right) \approx \exp \left(i t \hat{\mathcal{L}}_{+}\right)$. While this is difficult to prove in general, in the present case it does not appear to be unreasonable as $\tilde{\mathcal{L}}_{+}$describes a dynamics that differs from the full dynamics only in so far as the momentum of the tagged particle remains fixed. I expect this to be a largely irrelevant modification for long times, indeed.

The last step allows to identify the dynamic correlation functions above with the coherent scattering function, $\phi(k, t)$, and its incoherent counterpart, $\phi^{s}(p, t)$,

$$
\left\langle\rho_{k} \mid \exp \left(i t \mathcal{Q} \hat{\mathcal{L}}_{+} \mathcal{Q}\right) \rho_{k}\right\rangle\left\langle\rho_{p}^{s} \mid \exp \left(i t \mathcal{Q} \hat{\mathcal{L}}_{+} \mathcal{Q}\right) \rho_{p}^{s}\right\rangle \approx S_{k} \phi(k, t) \phi^{s}(p, t)
$$

With the three approximations introduced above, namely (i) the restriction to a subspace with the projector $\mathcal{P}_{\rho}$; (ii) the factorization of the resulting four-point function and (iii) the replacement $\tilde{\mathcal{L}}_{+} \rightarrow \hat{\mathcal{L}}_{+}$which together constitute the mode coupling approximation, one finds

$$
M_{v}(t) \approx M_{v \rho}(t)=\frac{m}{3 T} \sum_{k, \boldsymbol{p}} \mathcal{V}_{\rho}^{2}(\boldsymbol{k}, \boldsymbol{p}) S_{k} \phi(k, t) \phi^{s}(p, t),
$$


where the vertex, $\mathcal{V}_{\rho}(\boldsymbol{k}, \boldsymbol{p})$, is given by

$$
\mathcal{V}_{\rho}(\boldsymbol{k}, \boldsymbol{p})=N\left\langle\boldsymbol{v}_{s} \mid \mathcal{Q} \hat{\mathcal{L}}_{+} \rho_{k} \rho_{p}^{s}\right\rangle / S_{k}=N\left\langle\rho_{k} \rho_{p}^{s} \mid \mathcal{Q} \hat{\mathcal{L}}_{+} \boldsymbol{v}_{s}\right\rangle / S_{k}
$$

and the left and right vertex are identical due to detailed balance (see section 2.2). Considering the defintion of the left vertex, one finds

$$
\left\langle\boldsymbol{v}_{s} \mid \mathcal{L}_{+} \mathcal{Q} \rho_{k} \rho_{p}^{s}\right\rangle=\left\langle\boldsymbol{v}_{s} \mid \mathcal{L}_{0} \rho_{k} \rho_{p}^{s}\right\rangle=k\left\langle\boldsymbol{v}_{s} \mid j_{k}^{L} \rho_{p}^{s}\right\rangle+p\left\langle\boldsymbol{v}_{s} \mid \rho_{k} j_{p}^{s L}\right\rangle .
$$

The remaining averages are not difficult to calculate (see appendix F.2.1 and yield

$$
V \mathcal{V}_{\rho}(\boldsymbol{k}, \boldsymbol{p})=\frac{T}{m} \boldsymbol{k} n c_{k} \delta(\boldsymbol{k}+\boldsymbol{p}),
$$

where the delta function expresses the momentum conservation of the hard sphere interaction.

Finally, we get a closed expression for the memory kernel,

$$
M_{v \rho}(t)=\frac{4 \pi}{3} \frac{T}{m} \frac{1}{n} \int_{0}^{\infty} d k k^{4}\left(n c_{k}\right)^{2} S_{k} \phi(k, t) \phi^{s}(k, t),
$$

or ,

$$
M_{v \rho}(t)=12 \sqrt{2} \pi^{3} \omega_{E}^{2} \varphi^{2} \chi^{3} \int_{0}^{\infty} d \tilde{k} \tilde{k}^{4}\left(n c_{\tilde{k}}\right)^{2} S_{\tilde{k}} \phi(\tilde{k}, t) \phi^{s}(\tilde{k}, t),
$$

with the dimensionless wave number $\tilde{k}=k \ell_{0}$. Remember that $\omega_{E}$ denotes the Enskog collision frequency [eq. (1.11)] and $\chi$ is the pair correlation function at contact.

As I am ultimately interested in the long time behavior, I will assume that the time evolution of the collective density is well described by hydrodynamics, $\phi(k, t) \equiv \phi_{0}(k, t)=\exp \left(-D_{S} k^{2} t\right) \cos (c k t)$, where from now on I will assume that the temperature is constant in space and time for simplicity. Consistently with the hydrodynamic expression for the coherent scattering function, $\phi(k, t)$, I assume a diffusive behavior for the incoherent scattering function, $\phi^{s}(p, t) \equiv \phi_{0}^{s}(p, t)=$ $\exp \left(-D p^{2} t\right)$, where $D$ is the coefficient of diffusion.

From the Percus-Yevick approximation, one finds that $k^{4}\left(n c_{k}\right)^{2} S_{k} \simeq \cos ^{2}(2 a k)$ asymptotically for large $k$ (see Fig. 5.1). With $\phi(k, t=0)=\phi^{s}(k, t=0)=1$, this implies that the short time limit of the mode coupling memory kernel, $M_{v \rho}(t \rightarrow$ $0) \propto \int_{0}^{\infty} \cos ^{2}(2 k a) d k$, does not exist. Quite to the contrary, the memory kernel must vanish for short times, $M_{v}(t=0)=0$, because even the smallest ring collision involves at least three particles and two collisions with a finite interval of free streaming in between.

More careful derivations [260 264] find that, instead of $\phi(q, t) \phi^{s}(q, t)$, the memory kernel contains a term $\left[\phi(q, t)-\phi_{f}(q, t)\right] \phi^{s}(q, t)$, where $\phi_{f}(q, t)=\exp \left(-T k^{2} t^{2} / m\right)$ is the coherent scattering function of noninteracting particles. As, $\phi(q, t \rightarrow 0)=$ 

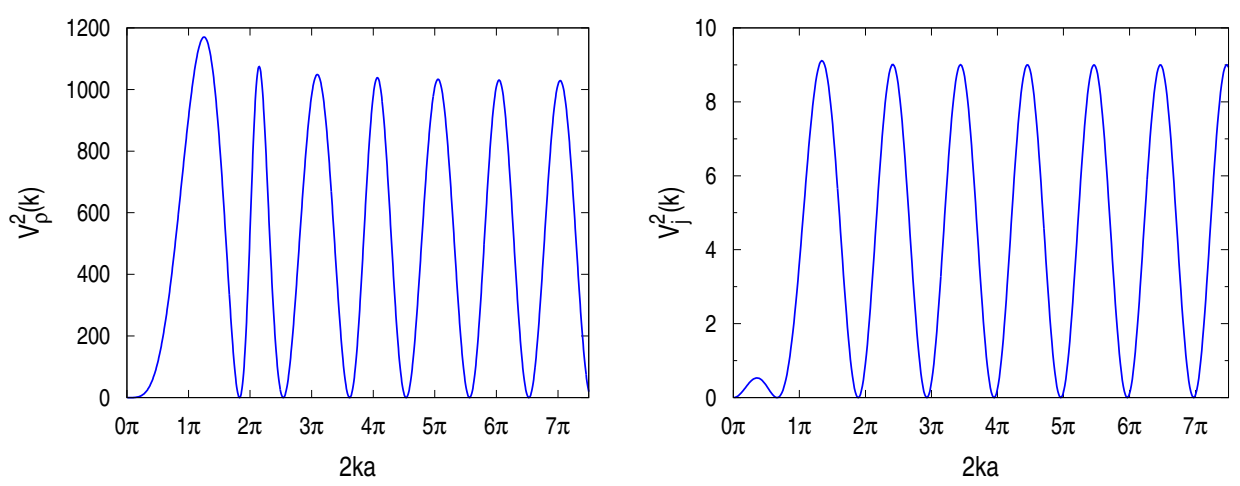

Figure 5.1: Left: The nontrivial wave number dependence, $V_{\rho}^{2}(k):=k^{4}\left(n c_{k}\right)^{2} S_{k}$, of the integral kernel of the mode coupling memory kernel $M_{v \rho}(t)$, eq. $[5.12$. Right: The same for the mode coupling memory kernel $M_{v \ell}$, i.e., $V_{j}^{2}(k):=k^{2}\left[2 j_{2}(2 a k)-j_{0}(2 a k)\right]$.

$\phi_{f}(q, t \rightarrow 0)$, the difference term vanishes for $t \rightarrow 0$ as it should. The behavior of the long time tails is independent of these considerations and thus I will continue to use the simple memory kernel derived above.

As both the static structure factor, $S_{k}$ as well as the direct correlation function, $c_{k}$, tend to a constant for $k \rightarrow 0$, one finds the following asymptotic form of the mode coupling memory kernel

$$
\begin{aligned}
M_{v \rho}(t \rightarrow \infty) & \propto \int_{0}^{\infty} d \tilde{k} \tilde{k}^{4} \exp \left[-\left(\tilde{D}+\tilde{D}_{S}\right) \tilde{k}^{2} t\right] \cos (c \tilde{k} t) \\
& \propto t^{-1 / 2} \exp \left[-c^{2} t / 4\left(D+D_{S}\right)\right]
\end{aligned}
$$

That this does not explain the long time tails was to be expected. Instead, in analogy to the mode coupling description of the incoherent scattering function, $\phi^{s}(q, t)$, (see chapter 7), I expect that $M_{v \rho}(t)$ describes the negative part of the VCAF. A negative region in the VACF is indicative of back scattering which in turn is a precursor of the cage effect.

Before coming back to question of long time tails, let me first show that the mode coupling approximation explained above can be generalized to the nonequilibrium stationary state of a driven granular fluid. After I have shown that this is indeed possible, I will introduce more mode coupling contributions to the memory kernel that will eventually be able to describe the long time tails.

\subsection{Mode Coupling Theory for a Driven Granular Fluid}

The Mori-Zwanzig expansion (appendix E) of the stationary state time evolution of a driven granular fluid in terms of a relaxation frequency, $\omega_{0}$, and a memory 
kernel, $M_{v}^{\epsilon}(t)$, is formally identical to that of an equilibrium fluid discussed above. The introduction of the projection operator $\left.\mathcal{P}=m\left|\boldsymbol{v}_{s}\right\rangle\right\rangle\left\langle\boldsymbol{v}_{s}\right| / 3 T$ yields a reduced relaxation frequency [67]

$$
\frac{m}{3 T}\left\langle\left\langle\boldsymbol{v}_{s} \mid i \mathcal{L}_{+} \boldsymbol{v}_{s}\right\rangle=\frac{1+\epsilon}{2} \omega_{E},\right.
$$

smaller than the elastic Enskog collision frequency, $\omega_{E}$, and a memory kernel

$$
M_{v}^{\epsilon}(t)=\frac{m}{3 T}\left\langle\left\langle\boldsymbol{v}_{s} \mid \mathcal{L}_{+} \mathcal{Q} \exp \left(i t \mathcal{Q} \mathcal{L}_{+} \mathcal{Q}\right) \mathcal{Q} \mathcal{L}_{+} \boldsymbol{v}_{s}\right\rangle\right\rangle
$$

where the dynamics is encoded in the granular pseudo Liouville operator, $\mathcal{L}_{+}(2.26)$, and the average $\langle\langle\cdot\rangle\rangle$ is defined over the stationary distribution function, $\varrho(\Gamma)(2.31$, and the realizations of the random driving force. With $\boldsymbol{F}^{\dagger}=\mathcal{Q} \mathcal{L}_{+}^{\dagger} \boldsymbol{v}_{s} \neq \mathcal{Q} \mathcal{L}_{+} \boldsymbol{v}_{s}=\boldsymbol{F}$, the memory kernel is no longer an autocorrelation of the fluctuating force $\boldsymbol{F}$ but the crosscorrelation function of two fluctuating forces, $\boldsymbol{F}$ and $\boldsymbol{F}^{\dagger}$, i.e., $M_{v}^{\epsilon}(t) \propto\left\langle\left\langle\boldsymbol{F}^{\dagger} \mid \boldsymbol{F}(t)\right\rangle\right.$.

The mode coupling projector

$$
\left.\mathcal{P}_{\rho}=N \sum_{\boldsymbol{k}, \boldsymbol{p}}\left|\rho_{k} \rho_{p}^{s}\right\rangle\right\rangle\left\langle\left\langle\rho_{k} \rho_{p}^{s}\right| / S_{k},\right.
$$

together with the mode coupling approximations discussed above yields the approximation

$$
M_{v}^{\epsilon}(t) \approx M_{v \rho}^{\epsilon}(t)=\frac{m}{3 T} \sum_{\boldsymbol{k}, \boldsymbol{p}} \mathcal{V}_{\rho}(\boldsymbol{k}, \boldsymbol{p}) \mathcal{V}_{\rho}^{\dagger}(\boldsymbol{k}, \boldsymbol{p}) \phi(k, t) \phi^{s}(p, t)
$$

with two distinct vertices $\mathcal{V}_{\rho}(\boldsymbol{k}, \boldsymbol{p})$ and $\mathcal{V}_{\rho}^{\dagger}(\boldsymbol{k}, \boldsymbol{p})$ due to the violation of detailed balance.

With $\mathcal{L}_{D}^{+} \rho_{k} \rho_{p}^{s}=0$, the left vertex, $\mathcal{V}_{\rho}=N\left\langle\left\langle\boldsymbol{v}_{s} \mid \mathcal{L}_{+} \mathcal{Q} \rho_{k} \rho_{p}^{s}\right\rangle / S_{k}\right.$, is formally given by eq. 5.11). The right vertex, $\mathcal{V}_{\rho}^{\dagger}(\boldsymbol{k}, \boldsymbol{p})=N\left\langle\left\langle\rho_{k} \rho_{p}^{s} \mathcal{Q} \mid \mathcal{L}_{+} \boldsymbol{v}_{s}\right\rangle\right\rangle / S_{k}$, can be calculated directly. Knowledge of the adjoint operator $\mathcal{L}_{+}^{\dagger}$ is not required. With the approximate granular YBG theorem 2.25 one finds (see appendix F.2.1),

$$
V \mathcal{V}_{\rho}^{\dagger}(\boldsymbol{k}, \boldsymbol{p})=\frac{1+\epsilon}{2} \frac{T}{m} \boldsymbol{k} n c_{k} \delta(\boldsymbol{k}+\boldsymbol{p}),
$$

smaller than the left vertex, $\mathcal{V}_{\rho}(\boldsymbol{k}, \boldsymbol{p})$. The random driving force suppresses the generation of density fluctuations through collisions between the tagged particle and the particles of the host fluid. The result $\mathcal{V}_{\rho}(\boldsymbol{k}, \boldsymbol{p}) \neq \mathcal{V}_{\rho}^{\dagger}(\boldsymbol{k}, \boldsymbol{p})$ supports the hypothesis that the granular pseudo Liouville operator $\mathcal{L}_{+}$has a nontrivial adjoint, $\mathcal{L}_{+}^{\dagger} \neq \mathcal{L}_{-}$ (see section 2.3).

For the momentum conserving driving considered here, the static structure factor of the driven granular fluid, $S_{k}$, although it is different from the one for a hard sphere fluid in thermal equilibrium, also tends to a constant value for $k \rightarrow 0$. Therefore, the asymptotic behavior of the memory kernel is the same as for an elastic hard sphere fluid, eq. 5.14). The strength of backscattering is reduced by a factor $(1+\epsilon) / 2$, though. 


\subsection{Long Time Tails}

Continuing the search for the origin of the long time tails, I introduce two more mode coupling contributions that describe the coupling of the tagged particle to the longitudinal, $j_{k}^{L}=\frac{1}{N} \sum_{j}\left(\hat{\boldsymbol{k}} \cdot \boldsymbol{v}_{j}\right) e^{i \boldsymbol{k} \cdot \boldsymbol{r}_{j}}$, and transverse, $\boldsymbol{j}_{k}^{T}=\boldsymbol{j}_{k}-\hat{\boldsymbol{k}} j_{k}^{L}$, current, respectively. Here, $\boldsymbol{j}_{k}=\frac{1}{N} \sum_{j} \boldsymbol{v}_{j} e^{i \boldsymbol{k} \cdot \boldsymbol{r}_{j}}$, is the collective current mode. To this end I define the projectors

$$
\begin{aligned}
\mathcal{P}_{\ell} & \left.=N \frac{m}{T} \sum_{\boldsymbol{k}, \boldsymbol{p}}\left|j_{k}^{L} \rho_{p}^{s}\right\rangle\right\rangle\left\langle\left\langle j_{k}^{L} \rho_{p}^{s}\right|,\right. \\
\mathcal{P}_{\perp} & \left.=N \frac{m}{2 T} \sum_{\alpha} \sum_{\boldsymbol{k}, \boldsymbol{p}}\left|j_{k}^{T \alpha} \rho_{p}^{s}\right\rangle\right\rangle\left\langle j_{k}^{T \alpha} \rho_{p}^{s}\right|,
\end{aligned}
$$

which lead to the following mode coupling memory kernels,

$$
M_{v \ell}^{\epsilon}(t)=\frac{m}{3 T} \sum_{\boldsymbol{k}, \boldsymbol{p}} \mathcal{V}_{\ell}(\boldsymbol{k}, \boldsymbol{p}) \mathcal{V}_{\ell}^{\dagger}(\boldsymbol{k}, \boldsymbol{p}) \phi^{L}(k, t) \phi(p, t)
$$

and

$$
M_{v \perp}^{\epsilon}(t)=\frac{m}{3 T} \sum_{\alpha, \beta} \sum_{\boldsymbol{k}, \boldsymbol{p}} \mathcal{V}_{\perp, \alpha \beta}(\boldsymbol{k}, \boldsymbol{p}) \mathcal{V}_{\perp, \alpha \beta}^{\dagger}(\boldsymbol{k}, \boldsymbol{p}) \phi^{T}(k, t) \phi^{s}(p, t),
$$

where $\phi^{L}(k, t)=m\left\langle\left\langle j_{k}^{L} \mid j_{k}^{L}(t)\right\rangle / T\right.$ and $\phi^{T}(k, t)=m\left\langle\left\langle\boldsymbol{j}_{k}^{T} \mid \boldsymbol{j}_{k}^{T}(t)\right\rangle\right\rangle / 2 T$ are the longitudinal and transverse current correlators, respectively.

In this case the left and right vertices turn out to be identical,

$$
\begin{aligned}
& \begin{array}{r}
V \mathcal{V}_{\ell}^{\dagger}(\boldsymbol{k}, \boldsymbol{p})=V \mathcal{V}_{\ell}(\boldsymbol{k}, \boldsymbol{p})=N m\left\langle\left\langle\boldsymbol{v}_{s} \mid \mathcal{L}_{+} \mathcal{Q} j_{k}^{L} \rho_{p}^{s}\right\rangle / T\right. \\
=\frac{1+\epsilon}{2} \omega_{E} \hat{\boldsymbol{k}}\left[2 j_{2}(2 a k)-j_{0}(2 a k)\right] \delta(\boldsymbol{k}+\boldsymbol{p}) \\
V \mathcal{V}_{\perp, \alpha \beta}^{\dagger}(\boldsymbol{k}, \boldsymbol{p})=V \mathcal{V}_{\perp, \alpha \beta}(\boldsymbol{k}, \boldsymbol{p})=N m\left\langle\left\langle v_{s}^{\alpha} \mid \mathcal{L}_{+} \mathcal{Q} j_{k}^{T \beta} \rho_{p}^{s}\right\rangle / T\right. \\
=\left(\delta_{\alpha \beta}-\hat{k}_{\alpha} \hat{k}_{\beta}\right) \frac{1+\epsilon}{2} \omega_{E}\left[2 j_{2}(2 a k)-j_{0}(2 a k)\right] \delta(\boldsymbol{k}+\boldsymbol{p})
\end{array}
\end{aligned}
$$

and are given in terms of the spherical Bessel functions, $j_{\nu}(x)$, of order $\nu$ [292] (see Fig. 5.1p. For the longitudinal vertices $\mathcal{V}_{\ell}(\boldsymbol{k}, \boldsymbol{p})$ and $\mathcal{V}_{\ell}^{\dagger}(\boldsymbol{k}, \boldsymbol{p})$, the nontrivial static correlations, $S_{\ell \ell}(k)$, discussed in chapter 3 above have been neglected.

With $\lim _{k \rightarrow 0}\left[2 j_{2}(2 a k)-j_{0}(2 a k)\right]=1$ and $\phi^{T}(k, t)=\exp \left(-\nu_{\perp} k^{2} t\right)$, the transverse contribution finally yields a memory kernel that is able to describe the long time tails,

$$
M_{v \perp}^{\epsilon}(t \rightarrow \infty) \propto \int_{0}^{\infty} d \tilde{k} \tilde{k}^{2} \exp \left[-\left(\tilde{\nu}_{\perp}+\tilde{D}\right) \tilde{k}^{2} t\right] \propto 1 /\left[\left(D+\nu_{\perp}\right) t\right]^{3 / 2},
$$


exactly like for elastic hard spheres. The magnitude of the memory kernel is reduced by a factor $(1+\epsilon)^{2} / 4$, though, which is again due to the randomizing effects of volume driving.

Finally, the sum of the mode coupling memory kernels, $M_{v}^{\epsilon}(t) \approx M_{v \rho}^{\epsilon}(t)+M_{v \ell}^{\epsilon}(t)+$ $M_{v \perp}^{\epsilon}(t)$, is expected to be a accurate description of the VACF of a driven granular fluid on macroscopic time scales.

\subsection{Discussion}

The mode coupling treatment of the VACF for elastic hard sphere fluids showed that it is one of the simplest mode coupling theories with some predictive power. Therefore it seemed to be a good starting point for the generalization of mode coupling theory to the stationary state of a dissipative fluid far from equilibrium. In the preceding sections I showed that indeed there are no fundamental obstacles to such a generalization. In particular, I was able to show that the long time tail, $\psi(t) \propto t^{-3 / 2}$, in a driven granular fluid has exactly the same origin as in a fluid in thermal equilibrium. In both situations the coupling of the tagged particles' velocity to the transverse current, $\boldsymbol{j}_{k}^{T}$, is responsible for the slow decay of the correlations.

It is tempting to try to calculate the coefficient of diffusion, $D$, self consistently via the Green-Kubo expression, $D \propto \int_{0}^{\infty} \psi(t) d t$. Unfortunately, this integral is dominate by the short time behavior of the VACF despite the long time tails. Therefore one would need a rather precise understanding of the short time evolution of the VACF. Naturally, this can not be given by mode coupling theory. A numerical solution of the equation of motion for the VACF is work in progress.

While I concentrated on the locally momentum conserving driving, it would also be interesting to get a mode coupling description of the modified long time tail, $\psi(t) \propto t^{-1}$, in the case of naive volume driving [67]. A similar situation is given by the Lorentz gas, where a tagged particle interacts with randomly placed fixed obstacles. The corresponding mode coupling theory [293] might therefore be relevant. 



\section{Granular Mode Coupling Theory ${ }^{1}$}

In this chapter I want to discuss the mode coupling description of the kinetic glass transition. I will start by describing the established projection operator approach in section 6.1 and then explain the modifications that appear if this approach is applied to the driven granular fluid in section 6.2. As the granular mode coupling theory is still in an early stage of development, I will complement the discussion of the first results (section 6.3) by an extended discussion of possible future directions (section 6.4).

\subsection{Mode-Coupling and the Glass Transition of Elastic Hard Spheres}

The results obtained in this section for a elastic hard sphere fluid in thermal equilibrium are a simplified version of the analysis by Leutheusser [278]. Technically, I follow the formalism by Bosse et al. [275].

Like in the preceding chapter, I want to use the projection operator approach to formulate the Langevin equation (4.6) for the coherent scattering function, $\phi(q, t)$, and the mode coupling memory kernel, eq. 4.17). To this end, I introduce the following projection operator,

$$
\mathcal{P}=N \sum_{q}\left|\rho_{q}\right\rangle\left\langle\rho_{q}\left|/ S_{q}+N m \sum_{q}\right| j_{q}^{L}\right\rangle\left\langle j_{q}^{L}\right| / T
$$

and use it to calculate correlation functions of the microscopic state

$$
\boldsymbol{A}_{q}=\sqrt{N}\left(\rho_{q} / \sqrt{S_{q}}, \sqrt{m / T} j_{q}^{L}\right) .
$$

What I ultimately want to know is the dynamic scattering function, $\phi(q, t)=$ $N\left\langle\rho_{q} \mid \rho_{q}(t)\right\rangle / S_{q}$, which is the 11-component of the matrix of correlation functions, $\phi_{\alpha \beta}(q, t)=\left\langle A_{q}^{\alpha} \mid A_{q}^{\beta}(t)\right\rangle$. In the Laplace domain this is equivalent to

$$
\hat{\phi}_{\alpha \beta}(s)=\left\langle A_{q}^{\alpha} \mid[s-\Omega-\mathrm{M}(s)]^{-1} A_{q}^{\beta}\right\rangle
$$

\footnotetext{
${ }^{1} \mathrm{~A}$ brief account of the subject of this chapter has been published in Ref. 294.
} 
where, according to the Mori-Zwanzig formalism, $\Omega=\mathcal{P} \hat{\mathcal{L}}_{+} \mathcal{P}, \mathrm{M}(s)=\mathcal{P} \hat{\mathcal{L}}_{+} \mathcal{Q}(s-$ $\left.\mathcal{Q} \hat{\mathcal{L}}_{+} \mathcal{Q}\right)^{-1} \mathcal{Q} \hat{\mathcal{L}}_{+} \mathcal{P}$ and $\mathcal{Q}=1-\mathcal{P}$. Inverting

$$
\left\langle\boldsymbol{A}_{q} \mid(s-\Omega-\mathrm{M}(s)) \boldsymbol{A}_{q}\right\rangle=N\left(\begin{array}{cc}
s / N & -\Omega_{\rho j} / \sqrt{T S_{q} / m} \\
-\Omega_{j \rho} / \sqrt{T S_{q} / m} & s / N-m \Omega_{j j} / T-m M_{j j}(s) / T
\end{array}\right),
$$

where all but the 22-component of $\mathrm{M}(s)$ vanish because $\mathcal{Q} \hat{\mathcal{L}}_{+} \rho_{q}=0$, one finds

$$
\begin{aligned}
& \left\langle\boldsymbol{A}_{q} \mid\left(s-\hat{\mathcal{L}}_{+}\right)^{-1} \boldsymbol{A}_{q}\right\rangle \\
= & \frac{1}{s\left[s-\frac{m N \Omega_{j j}}{T}-\frac{m N M_{j j}(s)}{T}\right]-\frac{m N^{2} \Omega_{\rho j} \Omega_{j \rho}}{T S_{q}}}\left(\begin{array}{cc}
s-\frac{m N \Omega_{j j}}{T}-\frac{m N M_{j j}(s)}{T} & \frac{N \Omega_{\rho j}}{\sqrt{T S_{q} / m}} \\
\frac{N \Omega_{j \rho}}{\sqrt{T S_{q} / m}} & s
\end{array}\right),
\end{aligned}
$$

where I used the notation $\Omega_{j j}=\left\langle j_{q}^{L} \mid \Omega j_{q}^{L}\right\rangle, \Omega_{\rho j}=\Omega_{j \rho}=\left\langle\rho_{q} \mid \Omega j_{q}^{L}\right\rangle$ and $M_{j j}(s)=$ $\left\langle A_{q}^{2} \mid \mathrm{M}(s) A_{q}^{2}\right\rangle$ and suppressed most of the wave number dependence. Due to parity, $\Omega_{\rho \rho}=\left\langle\rho_{q} \mid \hat{\mathcal{L}}_{+} \rho_{q}\right\rangle=q\left\langle\rho_{q} \mid j_{q}^{L}\right\rangle=0$ vanishes. In particular one finds

$$
\hat{\phi}(q, s)=N\left\langle\rho_{q} \mid\left(s-\hat{\mathcal{L}}_{+}\right)^{-1} \rho_{q}\right\rangle / S_{q}=\frac{1}{s-\frac{\Omega_{\rho j} \Omega_{j \rho} N^{2} / v_{0}^{2} S_{q}}{s-\Omega_{j j} N / v_{0}^{2}-M_{j j}(s) N / v_{0}^{2}}} .
$$

This can be written more transparently as

$$
\hat{\phi}^{-1}(q, s)=s-\frac{\Omega_{q}^{2}}{s-i \nu_{q}-M(q, s)},
$$

where

$$
\begin{aligned}
\Omega_{q}^{2} & =m N^{2} \Omega_{\rho j} \Omega_{j \rho} / T S_{q}=-q^{2} T / m S_{q}, \\
\nu_{q} & =m N \Omega_{j j} / T=\omega_{E}\left[1-j_{0}(2 a q)+2 j_{2}(2 a q)\right],
\end{aligned}
$$

with the Enskog frequency $\omega_{E}$ and the spherical Bessel functions $j_{\nu}(x)$ [292]. The relaxation rates $\left\langle\rho_{q} \mid \hat{\mathcal{L}}_{+} j_{q}^{L}\right\rangle=\left\langle j_{q}^{L} \mid \hat{\mathcal{L}}_{+} \rho_{q}\right\rangle=q\left\langle j_{q}^{L} \mid j_{q}^{L}\right\rangle=q T / m$ are easily determined. More details on the explicit calculation of the term $\nu_{q}$ can be found in appendix F.1. By Laplace transforming the equation of motion (4.6), one can show that it yields exactly the form of eq. 6.7) above.

The instantaneous part of the memory kernel, $\nu_{q}$, is sometimes called a viscosity. In fact, the coherent scattering function of damped sound waves described by a speed of sound, $c(q)$, and a viscosity, $\zeta_{q}$, reads

$$
\hat{\phi}^{-1}(q, s)=s-\frac{q^{2} c^{2}(q)+q^{4} \zeta_{q}^{2}}{s-2 i q^{2} \zeta_{q}-\hat{M}(q, s)} .
$$


This implies that, more precisely, $\zeta_{q} \equiv \nu_{q} / 2 q^{2}$ is a viscous term. To lowest order in $q$ one finds $\zeta_{q}=3 a^{2} \omega_{E} / 5+\mathcal{O}\left(q^{2}\right)$. The speed of sound is then given by

$$
c^{2}(q)=\frac{\Omega_{q}^{2}}{q^{2}}-q^{2} \zeta_{q}^{2},
$$

where the viscous correction is of the order $q^{2}$ and does not alter the long wavelength speed of sound, $\lim _{q \rightarrow 0} c^{2}(q)=T / m S(q=0)$.

For the memory kernel, $\hat{M}(q, s)=m N M_{j j}(q, s) / T$, I am going to use the mode coupling approximation discussed above (section 5.1). To this end I define the projector

$$
\mathcal{P}_{2}=N^{2} \sum_{\boldsymbol{k}, \boldsymbol{p}}\left|\rho_{k} \rho_{p}\right\rangle\left\langle\rho_{k} \rho_{p}\right| / S_{k} S_{p}
$$

and write

$$
\begin{aligned}
M_{j j}(q, t) & =\left\langle j_{q}^{L} \mid \hat{\mathcal{L}}_{+} \mathcal{Q} \exp \left(i t \mathcal{Q} \hat{\mathcal{L}}_{+} \mathcal{Q}\right) \mathcal{Q} \hat{\mathcal{L}}_{+} j_{q}^{L}\right\rangle \\
& \approx\left\langle j_{q}^{L} \mid \hat{\mathcal{L}}_{+} \mathcal{Q} \mathcal{P}_{2} \exp \left(i t \mathcal{Q} \hat{\mathcal{L}}_{+} \mathcal{Q}\right) \mathcal{P}_{2} \mathcal{Q} \hat{\mathcal{L}}_{+} j_{q}^{L}\right\rangle .
\end{aligned}
$$

After the factorization step and with all the normalization factors included, this yields

$$
M(q, t) \approx M^{\mathrm{MCT}}(q, t)=\frac{m}{T} N^{3} \sum_{k, p}\left\langle j_{q}^{L} \mid \hat{\mathcal{L}}_{+} \mathcal{Q} \rho_{k} \rho_{p}\right\rangle\left\langle\rho_{k} \rho_{p} \mathcal{Q} \mid \hat{\mathcal{L}}_{+} j_{q}^{L}\right\rangle \phi(k, t) \phi(p, t) / S_{k} S_{p}
$$

For attempts to avoid the factorization of the four point function, see Refs. [295297]. Alternatively, the factorization can be understood as a kind of mean-field approximation, neglecting fluctuations of the 'order parameter' [32]. Indeed, if we regard the scattering function, $\phi(q, t)=\langle\Phi(q, t)\rangle$, as the macroscopic value of a microscopic 'order parameter', $\Phi(q, t)=\rho_{q}(0)^{*} \rho_{q}(t)$, then the factorization assumption corresponds to setting the fluctuations, $\Delta \phi(k, p, t)=\langle\Phi(k, t) \Phi(p, t)\rangle-$ $\langle\Phi(k, t)\rangle\langle\Phi(p, t)\rangle$, to zero.

The vertices, $\mathcal{V}_{q k p}=N\left\langle j_{q}^{L} \mid \hat{\mathcal{L}}_{+} \mathcal{Q} \rho_{k} \rho_{p}\right\rangle / S_{k}=N\left\langle\rho_{k} \rho_{p} \mathcal{Q} \mid \hat{\mathcal{L}}_{+} j_{q}^{L}\right\rangle / S_{p}$ can be reduced to static correlation functions explicitly (see appendix F.2.3),

$$
V \mathcal{V}_{\boldsymbol{q} k \boldsymbol{p}}=\frac{1}{N} \frac{T}{m} S_{k}^{-1}\left[(\hat{\boldsymbol{q}} \cdot \boldsymbol{k}) S_{p}+(\hat{\boldsymbol{q}} \cdot \boldsymbol{p}) S_{k}-q S^{(3)}(\boldsymbol{k}, \boldsymbol{p}) / S_{q}\right] \delta(\boldsymbol{k}+\boldsymbol{p}-\boldsymbol{q}),
$$

where the delta function is a consequence of momentum conservation, again.

Unfortunately, much less is known about the triplet correlation function, $S^{(3)}(\boldsymbol{k}, \boldsymbol{p})$, than about the structure factor, $S_{q}$. For some results, see e.g., Refs. [298, 299]. Conventionally, the Kirkwood superposition approximation [300, 301, $S^{(3)}(\boldsymbol{k}, \boldsymbol{p}) \approx$ $S_{q} S_{k} S_{p}$, is used. While it is largely uncontrolled and was shown to fail on large 
length scales [302], it can be understood as the maximization of an approximate entropy functional [303].

Using the superposition approximation, the vertex can be expressed in terms of the direct correlation function, $n c_{q}=1-S_{q}^{-1}$,

$$
V \mathcal{V}_{\boldsymbol{q} k \boldsymbol{p}}=n \frac{1}{N} \frac{T}{m} S_{p}\left[(\hat{\boldsymbol{q}} \cdot \boldsymbol{k}) c_{k}+(\hat{\boldsymbol{q}} \cdot \boldsymbol{p}) c_{p}\right] \delta(\boldsymbol{k}+\boldsymbol{p}-\boldsymbol{q})
$$

Thus, the memory kernel of an elastic hard sphere fluid within the mode coupling and Kirkwood superposition approximation can be given explicitly in terms of the static spatial correlation functions as

$$
M[\phi](q, s)=\frac{T}{m} \frac{n^{2}}{N} \sum_{\boldsymbol{k}+\boldsymbol{p}=\boldsymbol{q}} S_{k} S_{p}\left[(\hat{\boldsymbol{q}} \cdot \boldsymbol{k}) c_{k}+(\hat{\boldsymbol{q}} \cdot \boldsymbol{p}) c_{p}\right]^{2} \phi(k, t) \phi(p, t) .
$$

Whenever I want to emphasis that the mode coupling memory kernel is expressed in terms of the correlation function $\phi$, I will write it with the functional argument explicitly. Going over to a infinite system we can write the dimensionless memory kernel, $m(q, t)=M(q, t) / \Omega_{q}^{2}$, as

$$
m[\phi](q, t)=\frac{n}{q^{2} S_{q}} \int d^{3} k S_{k} S_{|\boldsymbol{q}-\boldsymbol{k}|}\left\{[\hat{\boldsymbol{q}} \cdot \boldsymbol{k}] c_{k}+[\hat{\boldsymbol{q}} \cdot(\boldsymbol{q}-\boldsymbol{k})] c_{|\boldsymbol{q}-\boldsymbol{k}|}\right\}^{2} \phi(k, t) \phi(|\boldsymbol{q}-\boldsymbol{k}|, t) .
$$

The temperature does not appear in the above expression at all. This expresses the fact that the hard sphere system has no intrinsic energy scale and thus the temperature only sets the overall timescale of the system.

Apart from the viscous term $\nu_{q}$ that is peculiar to hard sphere fluids, the memory kernel for a fluid composed of particles with a differentiable potential has a formally identical mode coupling approximation [287.

\subsection{The Granular Glass Transition}

To get a theoretical description of a possible kinetic glass transition in a driven granular fluid, I want to generalize the mode coupling formalism outlined above to the nonequilibrium stationary state. The success of this program for the VACF in the preceding chapter shows that there is reason to expect that the coherent scattering function, $\phi(q, t)=N\left\langle\left\langle\rho_{q} \mid \rho_{q}(t)\right\rangle / S_{q}\right.$, of the driven granular fluid will be determined by an equation of motion that is formally identical to the one for a fluid in thermal equilibrium.

Moreover it may also be possible to apply the mode coupling approximation introduced above for elastic hard spheres. For the VACF it turned out that the lack of knowledge of the adjoint Liouville operator, $\mathcal{L}_{+}^{\dagger}$, made matters technically 
more involved but presented no fundamental problems. I will show below that the situation is more complicated for the coherent scattering function but that in the end, a granular mode coupling theory can still be formulated.

To get a prediction of a granular kinetic glass transition, granular mode coupling theory has to fulfill one more requirement in addition to the treatment of the VACF in the preceding chapter. If the bifurcation scenario, i.e, the discontinuous jump of the EA order parameter, $f_{q}$, at the critical density $\varphi_{c}$ is to remain valid, the approximate memory kernel derived by the granular mode coupling theory must be positive definite. I will show that this is guaranteed because the memory kernel of the driven granular fluid formally differs from equation (6.17) above by a positive prefactor only.

\subsubsection{The Equation of Motion}

To begin with, I define the equation of motion by introducing the projector

$$
\left.\mathcal{P}=N \sum_{q}\left|\rho_{q}\right\rangle\right\rangle\left\langle\langle \rho _ { q } | / S _ { q } + N m \sum _ { q } | j _ { q } ^ { L } \rangle \left\langle\left\langle j_{q}^{L}\right| / T\right.\right.
$$

and the microscopic state $\boldsymbol{A}_{q}$, equation $(6.2)$. Again, the average $\left.\langle\cdot \cdot\rangle\right\rangle$ is defined over the distribution function in the nonequilibrium stationary state, eq. (2.31), and the realizations of the stochastic driving force $\boldsymbol{\xi}$. The dynamics is encoded in the pseudo Liouville operator, $\mathcal{L}_{+}$, eq. 2.26 .

Without having an explicit expression for the adjoint Liouville operator, $\mathcal{L}_{+}^{\dagger}$, I can not rule out that the memory kernel $M_{\rho j}(q, t)=\left\langle\left\langle\rho_{q} \mid \mathcal{L}_{+} \mathcal{Q} \exp (i t \mathcal{Q} \mathcal{L} \mathcal{Q}) \mathcal{Q} \mathcal{L}_{+} j_{q}^{L}\right\rangle\right\rangle$ is actually nonzero. Following the procedure outlined in the preceding section, I therefore conclude that the coherent scattering function is given by

$$
\hat{\phi}^{-1}(q, s)=s-\frac{m N^{2} \Omega_{j \rho}\left[\Omega_{\rho j}+M_{\rho j}(s)\right] / T S_{q}}{s-m N \Omega_{j j} / T-m N M_{j j}(s) / T} .
$$

Within the mode coupling approximation discussed below, $M_{\rho j}^{\mathrm{MCT}}(q, s)=0$. In this case, the equation of motion for the coherent scattering function, $\phi(q, t)$, of the driven granular fluid is formally identical to the one for the elastic hard spheres (6.7).

For the relaxation rates one finds

$$
\Omega_{j \rho}=\left\langle\left\langle j_{q}^{L} \mid \mathcal{L}_{+} \rho_{q}\right\rangle\right\rangle,=\frac{q}{N} \frac{T}{m}
$$

identical to the elastic case but (see appendix F.1),

$$
\Omega_{\rho j}=\left\langle\left\langle\rho_{q} \mid \mathcal{L}_{+} j_{q}^{L}\right\rangle\right\rangle=\frac{q}{N} \frac{T}{m}\left[\frac{1+\epsilon}{2}+\frac{1-\epsilon}{2} S_{q}\right],
$$




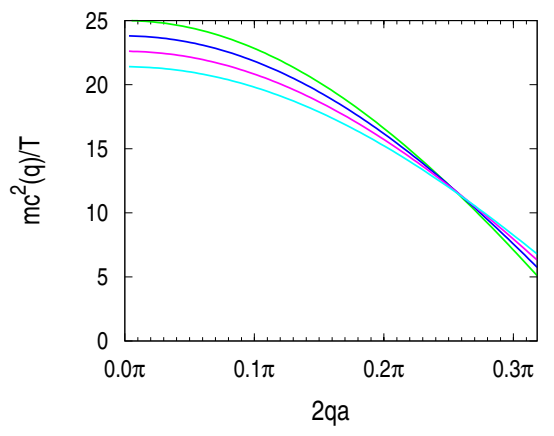

Figure 6.1: Speed of sound, $m c^{2}(q) / T$ as a function of wave number, $q$, at a volume fraction $\varphi=0.4$ for different values of the coefficient of restitution $\epsilon=1.0$ (green), 0.9 (dark blue), 0.8 (magenta), 0.7 (light blue).

different and explicitly dependent on the coefficient of restitution. Note the additional structure factor appearing here. It accounts for the fact that due to the inelasticity the granular particles have a higher probability to be close together compared to elastic particles. The hard sphere contribution,

$$
\Omega_{j j}=\left\langle\left\langle j_{q}^{L} \mid \mathcal{L}_{+} j_{q}^{L}\right\rangle\right\rangle=-i \omega_{E} \frac{1+\epsilon}{2 N} \frac{T}{m}\left[1-j_{0}(2 a q)+2 j_{2}(2 a q)\right],
$$

only acquires an $\epsilon$-dependent prefactor.

The viscosity,

$$
\zeta_{q}=\frac{1+\epsilon}{4} \omega_{E}\left[1-j_{0}(2 a q)+2 j_{2}(2 a q)\right] / q^{2},
$$

is thus also reduced compared to a fluid of elastic hard spheres in thermal equilibrium. For the speed of sound one finds

$$
c^{2}(q)=\frac{T}{m} \frac{1}{S_{q}}\left[\frac{1+\epsilon}{2}+\frac{1-\epsilon}{2} S_{q}\right]-q^{2} \zeta_{q}^{2} .
$$

For increasing dissipation, i.e., smaller coefficient of restitution $\epsilon$, the long wave length speed of sound decreases smoothly from its elastic value (see Fig. 6.1). This is in contrast to the hydrodynamic result [eq. (3.16)] which predicts a significantly lower speed of sound as soon as $\epsilon<1$. Temperature fluctuations and their coupling to the density fluctuations lead to the modified speed of sound in the hydrodynamic theory. They are completely neglected in the present theory, which, consequently, can not describe the discontinuous jump in the speed of sound upon introducing any inelasticity.

\subsubsection{Granular Mode Coupling Theory}

With the mode coupling approximation defined by the projector

$$
\left.\mathcal{P}_{2}=N^{2} \sum_{k, p}\left|\rho_{k} \rho_{p}\right\rangle\right\rangle\left\langle\left\langle\rho_{k} \rho_{p}\right| / S_{k} S_{p},\right.
$$


one finds

$$
\begin{aligned}
M_{\rho j}(q, t) & =\left\langle\left\langle\rho_{q} \mid \mathcal{L}_{+} \mathcal{Q} \exp \left(i t \mathcal{Q} \mathcal{L}_{+} \mathcal{Q}\right) \mathcal{Q} \mathcal{L}_{+} j_{q}^{L}\right\rangle\right\rangle \\
& \approx N^{2} \sum_{k, p}\left\langle\left\langle\rho_{q} \mid \mathcal{Q} \mathcal{L}_{+} \rho_{k} \rho_{p}\right\rangle\right\rangle\left\langle\left\langle\rho_{k} \rho_{p} \mid \mathcal{Q} \mathcal{L}_{+} j_{q}^{L}\right\rangle\right\rangle \phi(k, t) \phi(p, t) / S_{k} S_{p}=0,
\end{aligned}
$$

because $\left\langle\left\langle\rho_{q} \mid \mathcal{Q} \mathcal{L}_{+} \rho_{k} \rho_{p}\right\rangle\right\rangle=0$, confirming the assumption made above, and

$$
\begin{aligned}
M_{j j}(q, t) & =\left\langle\left\langle j_{q}^{L} \mid \mathcal{L}_{+} \mathcal{Q} \exp \left(i t \mathcal{Q L}_{+} \mathcal{Q}\right) \mathcal{Q} \mathcal{L}_{+} j_{q}^{L}\right\rangle\right. \\
& \approx N^{2} \sum_{k, p}\left\langle\left\langle j_{q}^{L} \mid \mathcal{Q} \mathcal{L}_{+} \rho_{k} \rho_{p}\right\rangle\right\rangle\left\langle\rho_{k} \rho_{p} \mid \mathcal{Q} \mathcal{L}_{+} j_{q}^{L}\right\rangle \phi(k, t) \phi(p, t) / S_{k} S_{p}
\end{aligned}
$$

For the left vertex we find

$$
\left\langle\left\langle j_{q}^{L} \mid \mathcal{L}_{+} \mathcal{Q} \rho_{k} \rho_{p}\right\rangle\right\rangle=\frac{T}{m} \frac{\delta(\boldsymbol{k}+\boldsymbol{p}-\boldsymbol{q})}{N^{2} V}\left[(\hat{\boldsymbol{q}} \cdot \boldsymbol{k}) S_{p}+(\hat{\boldsymbol{q}} \cdot \boldsymbol{p}) S_{k}-q S^{(3)}(\boldsymbol{k}, \boldsymbol{p}) / S_{q}\right]
$$

which is exactly the elastic result but the right vertex,

$$
\left\langle\left\langle\rho_{k} \rho_{p} \mathcal{Q} \mid \mathcal{L}_{+} j_{q}^{L}\right\rangle=-\frac{1+\epsilon}{2} \frac{T}{m} \frac{\delta(\boldsymbol{k}+\boldsymbol{p}-\boldsymbol{q})}{N^{2} V}\left[(\hat{\boldsymbol{q}} \cdot \boldsymbol{k}) S_{p}+(\hat{\boldsymbol{q}} \cdot \boldsymbol{p}) S_{k}-q S^{(3)}(\boldsymbol{k}, \boldsymbol{p}) / S_{q}\right]\right.
$$

explicitly depends on the coefficient of restitution again (see appendix F.2.3). With these results and upon applying the Kirkwood superposition approximation on conventional grounds, we find the memory kernel for the granular dynamics,

$$
M[\phi](q, s)=\frac{1+\epsilon}{2} \frac{T}{m} \frac{n^{2}}{N} \sum_{\boldsymbol{k}+\boldsymbol{p}=\boldsymbol{q}} S_{k} S_{p}\left[(\hat{\boldsymbol{q}} \cdot \boldsymbol{k}) c_{k}+(\hat{\boldsymbol{q}} \cdot \boldsymbol{p}) c_{p}\right]^{2} \phi(k, t) \phi(p, t),
$$

which is formally identical to the expression for the elastic case safe for the prefactor $(1+\epsilon) / 2$.

For the dimensionless memory kernel,

$$
m[\phi](q, t)=\frac{n A_{\epsilon}(q)}{q^{2} S_{q}} \int d^{3} k S_{k} S_{|\boldsymbol{q}-\boldsymbol{k}|}\left\{[\hat{\boldsymbol{q}} \cdot \boldsymbol{k}] c_{k}+[\hat{\boldsymbol{q}} \cdot(\boldsymbol{q}-\boldsymbol{k})] c_{|\boldsymbol{q}-\boldsymbol{k}|}\right\}^{2} \phi(k, t) \phi(|\boldsymbol{q}-\boldsymbol{k}|, t),
$$

this implies an $\epsilon$-dependent prefactor, $A_{\epsilon}(q)=\left[1+(1-\epsilon) S_{q} /(1+\epsilon)\right]^{-1}$. This is a quite remarkable result. First of all, it guarantees that the memory kernel remains positive definite. The effect of the prefactor $A_{\epsilon}(q)<1$ is to weaken the memory effects compared to the elastic case. To get a memory effect for a dissipative system comparable to the elastic case, one thus has to increase the density. This will make the structure factor peaks more pronounced and thereby leads to a larger contribution to the memory kernel. 
At this point we have established that the mode coupling formalism can be generalized to a genuinely far from equilibrium system. This is a fundamental result of this thesis.

It needed considerable mathematical and numerical ingenuity to, first, establish how to find the physically relevant solution of the mode coupling equation and, second, devise an efficient numerical scheme to actually solve it within reasonable time. It would be a formidable task to redo all these developments for the generalized, non-equilibrium theory I set up so far. Fortunately this is not necessary. The only formal change to the memory kernel is the strictly positive prefactor $A_{q}(\epsilon)$. This does not invalidate the positive definiteness of the memory kernel which has been crucial for the equilibrium theory. Consequently, all the mathematical results that appear in the literature for the mode coupling theory can readily be applied for our non-equilibrium extension. For the same reason also the numerical programs to solve the equations only need to be adjusted for the prefactors depending on the coefficient of restitution $\epsilon$.

\subsection{Discussion}

At this point it should be no surprise that we do find a granular glass transition. It is also virtually impossible that it will not depend on the coefficient of restitution, $\epsilon$. Furthermore if it depends on $\epsilon$ it will smoothly deviate from the elastic, equilibrium result. The only problem could be, that we are restricted to almost elastic particles because the critical density will attain unphysical values for stronger dissipation. For the EA order parameter, $f_{q}$, we expect a dependence on the coefficient of restitution, $\epsilon$. This precludes an universality of the long time dynamics among the different degrees of dissipation. The critical long time dynamics for different coefficients of restitution cannot be mapped on a single master curve by rescaling the time.

The numerical solution is based on a program by Dr. M. Sperl which we adapted to the granular system. To make the results comparable with the existing literature, I retained the choice of discretization parameters. In particular I used a wave number cutoff at $2 q_{\max } a=40$ and a discretization into $M=400$ grid points in reciprocal space. Time gets discretized on a logarithmic grid. Starting with $2^{11}$ time steps with a spacing of $\Delta t v_{0} / d=10^{-9}$ the time step gets doubled for the next $2^{11}$ steps and this procedure is repeated until the final time $t_{\max }$ is reached. More details on the efficient numerical algorithm to calculate the memory kernel can be found in Ref. 304].

Taking the elastic Percus-Yevick expression for the static structure factors for simplicity, a numerical estimation of the critical density shows that the theory predicts values that are not a priori unreasonable all the way down to $\epsilon \rightarrow 0$ (see Fig. 1.9). The increase of the critical packing fraction with increasing dissipation is to be 

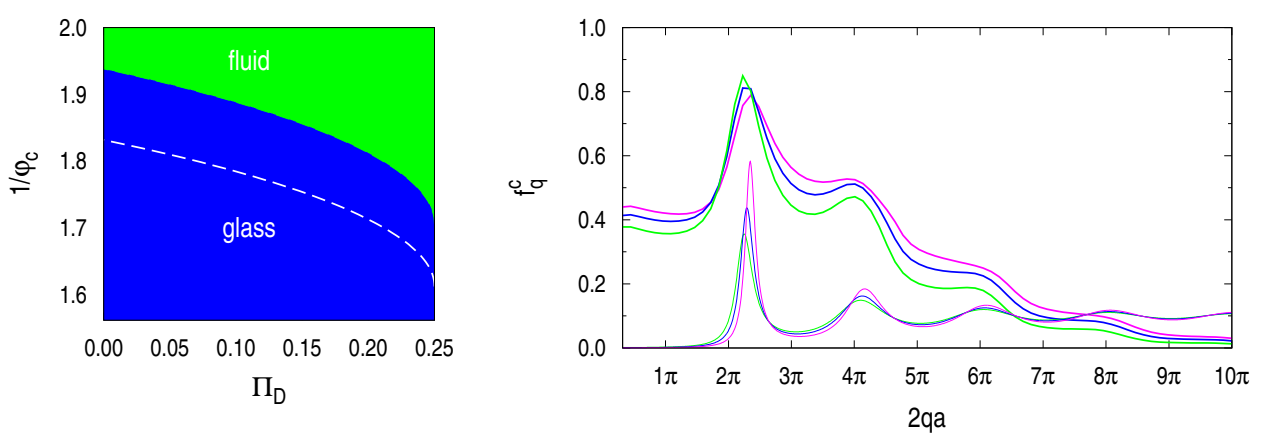

Figure 6.2: Left: Alternative rendering of the phase diagram. The inverse critical density, $1 / \varphi_{c}$, as a function of the dimensionless driving power, $\Pi_{D}$. The white dashed line is an extrapolation of the critical density corrected for the Percus-Yevick approximation in the limit of elastic hard spheres. Right: The EA order parameter at the critical density, $f_{q}^{c}$, as a function of the wave number, $q$, for different values of the coefficient of restitution $\epsilon=1.0$ (green), 0.5 (blue), 0.0 (magenta). The thin lines show the scaled Percus-Yevick structure factor, $S_{q} / 10$, for the corresponding critical densities, $\varphi_{c}(\epsilon)$.

expected insofar as the memory kernel for a given density is a decreasing function of $\epsilon$. Only at a higher density the memory effects are strong enough to trigger the bifurcation.

Instead of using the coefficient of restitution, $\epsilon$, as the control parameter, one can also use the dimensionless driving power, $\Pi_{D}=P_{D} / \omega_{0} T$. In the Enskog approximation of the stationary temperature, it is simply given by $\Pi_{D}=\left(1-\epsilon^{2}\right) / 4$. In this way, one could make closer contact to the jamming diagram (Fig. 1.3), where the external driving force, the shear stress, $\sigma$, is used instead of a quantity that would characterize the dissipation. Plotting $1 / \varphi_{c}$ versus $\Pi_{D}$ (Fig. 6.2 a), one gets a variant of the jamming diagram adapted to the case of a randomly driven granular fluid. The granular kinetic glass transition is the same for any nonzero temperature and thus, the temperature axis in the original jamming diagram can be neglected.

For a fluid of elastic hard spheres, it was found that MCT with the Percus-Yevick structure factor underestimates the critical density $\varphi_{c}$ by $\sim 5.8 \%$ [288]. Assuming that this holds also for the granular kinetic glass transition and the granular mode coupling theory, the dashed line in Fig. 6.2 a represents an estimate of the "true" granular glass transition.

While the granular kinetic glass transition defines the lower bound for the density of a granular glass, the upper bound is likely given by the random close packed density $\varphi_{\text {rcp }} \approx 0.64$. Consequently, I used $1 / \varphi_{\text {rcp }}$ as the lower bound in Fig. 6.2 a.

The corresponding critical EA oder parameters, $f_{q}^{c}$, are shown for a few values of the coefficient of restitution, $\epsilon$, in Fig. 6.2 $\mathrm{p}$. For all values of $\epsilon$, the EA order parameter 

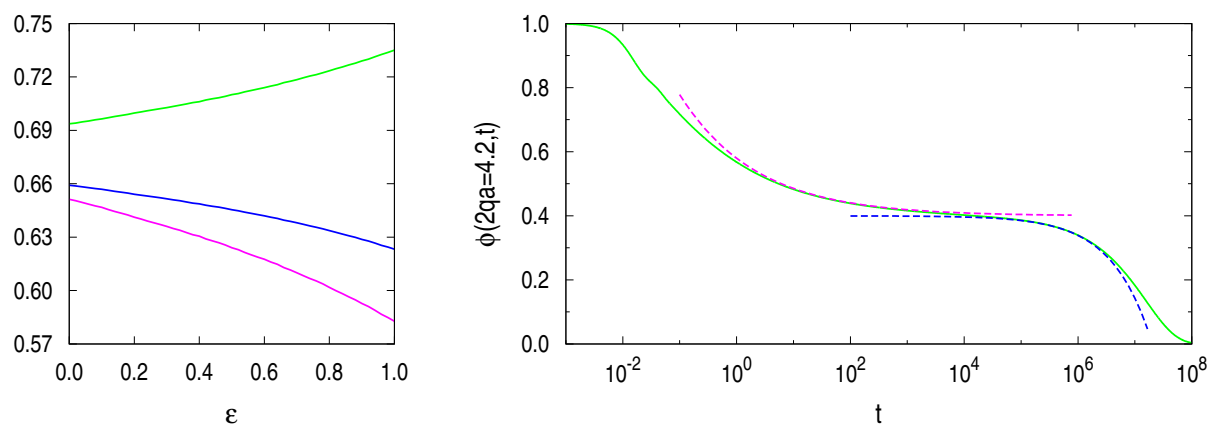

Figure 6.3: Left: The critical parameter, $\lambda$ (green), and the critical exponents, $2 a$ (blue) and $b$ (magenta), as a function of the coefficient of restitution, $\epsilon$. Right: The coherent scattering function, $\phi(q, t)$, close to the granular glass transition $\left(\Delta \varphi=10^{-4}\right)$ as a function of time for wave number $2 q a=4.2$ and coefficient of restitution $\epsilon=0.5$. The blue dashed line is the von Schweidler law with exponent $b$, while the magenta dashed line denotes the approximation for the $\beta$-relaxation with exponent $a$.

roughly follows the static structure factor. Upon increasing the inelasticity, $f_{q}^{c}$ attains higher values for most length scales, implying a stronger correlation between the corresponding density modes, except around the first peak, where it is slightly depressed compared to the result for an elastic hard sphere fluid.

As expected, the critical parameter, $\lambda$, and consequently also the critical exponents, $a$ and $b$, depend on the coefficient of restitution, $\epsilon$ (see Fig. 6.3p. Close to the glass transition, the three parameters $f_{q}^{c}, a$ and $b$ provide a good description of the coherent scattering function over several orders of magnitude in time (see Fig. 6.3).

\subsection{Perspectives}

The mathematical analysis of the mode coupling equations is quite advanced (see, Ref. [287]). The application of this analysis is work in progress and will yield a better understanding of the coherent scattering function close to the glass transition. The extraction of rheological quantities like the viscosity or the shear modulus [305], now all expected to be a function of the coefficient of restitution, will be a logical next step.

The granular mode coupling theory developed in this chapter shows explicitly that mode coupling theory can be applied to systems that are genuinely far from equilibrium. The integrating through transients (ITT) approach of Fuchs et al. [180] considers a system that is forced out of equilibrium by an applied shear but that is in thermal equilibrium initially. For the driven granular fluid, there is no such equilibrium reference state. Within the approach presented here, there no need for 
such a reference state. This gives me reason to believe that it will be applicable to a range of nonequilibrium systems other than driven granular fluids.

The rather formal approach to extend the ITT formalism to sheared granular fluids that was recently presented by Hayakawa and Otsuki [306], implicitly assumes detailed balance. If this is precisely verified or if this constitutes a crucial assumption is not clear to me at the moment. Oppenheim et al. also generalize their derivation of mode coupling theory [307, 308, to inelastically interacting, macroscopic particles [309]. Their approach both to mode coupling and to the interaction of granular particles is completely different from the one chosen here. This makes a comparison challenging but I expect it to be a worthwhile exercise.

With the possibility of a nonequlibrium granular mode coupling theory established a number of refinements present themselves as natural next steps. First of all granular MCT should be generalized to mixtures to make contact with experimental realizations. Following the work of Latz et al. [310, 311, I do not expect this will introduce any essentially new problems.

To avoid unnecessary complications, I chose a rather well established mode coupling formalism for my generalization to a granular mode coupling theory. Initial attempts to extend MCT focused on the description of hopping as a possible mechanism to eventually avoid the localization transition [312 316]. More recent approaches try to make the interpretation of MCT as a mean field theory more precise [317+320] or provide a more careful derivation from a field theoretic perspective [321, 322]. It would certainly be interesting to study some of this approaches with respect to their implications for a granular mode coupling theory. 



\section{Tagged Particle Dynamics Close to the Glass Transition}

In this chapter, I want to discuss the tagged particle dynamics close to the granular kinetic glass transition. Technically, the mode coupling theory for the incoherent scattering function, $\phi^{s}(q, t)$, is very similar to the mode coupling theory for the coherent scattering function, $\phi(q, t)$. In the next section, I will therefore only sketch the derivation of the granular mode coupling theory for the incoherent scattering function.

Partial information about the incoherent scattering function is contained in the mean square displacement, $\left\langle\delta r^{2}\right\rangle(t)$, the quantity that originally led to the proposition of a granular glass transition. In section 7.2 I will discuss granular mode coupling results both for the incoherent scattering function and the mean square displacement.

\subsection{Granular MCT for the Incoherent Scattering Function}

Via the Mori-Zwanzig projection operator technique, one first derives a generalized Langevin equation for the tagged particle density, $\rho_{q}^{s}=\exp \left(i \boldsymbol{q} \cdot \boldsymbol{r}_{s}\right)$, and longitudinal current, $j_{q}^{s L}=\left(\hat{\boldsymbol{q}} \cdot \boldsymbol{v}_{s}\right) e^{i \boldsymbol{q} \cdot \boldsymbol{r}_{s}}$. In particular, by applying the projector

$$
\left.\mathcal{P}^{s}=\sum_{k}\left|\rho_{k}^{s}\right\rangle\right\rangle\left\langle\left\langle\rho_{k}^{s}\left|+\frac{m}{T} \sum_{k}\right| j_{k}^{s L}\right\rangle\right\rangle\left\langle\left\langle j_{k}^{s L}\right|\right.
$$

to the state

$$
\boldsymbol{A}_{k}^{s}=\left(\rho_{k}, j_{k}^{L} \sqrt{m / T}\right)
$$

one finds,

$$
\begin{aligned}
\left\langle\left\langle\boldsymbol{A}_{k}^{s} \mid(s-\Omega-\mathrm{M}(s)) \boldsymbol{A}_{k}^{s}\right\rangle^{-1}\right. & \\
= & \frac{1}{s\left[s-\frac{m \Omega_{j j}^{s}}{T}-\frac{m M_{j j}^{s}(s)}{T}\right]-\frac{m \Omega_{\rho j}^{s} \Omega_{j \rho}^{s}}{T}}\left(\begin{array}{cc}
s-\frac{m \Omega_{j j}^{s}}{T}-\frac{m M_{j j}^{s}(s)}{T} & \sqrt{\frac{m}{T}} \Omega_{\rho j}^{s} \\
\sqrt{\frac{m}{T}} \Omega_{j \rho}^{s} & s
\end{array}\right),
\end{aligned}
$$

which looks superficially identical to the continued fraction for the coherent quantities. One should regard the inclusion of the tagged particle current, $j_{q}^{s L}$, in the set of 
macroscopic observables although it is not a conserved quantity as a technical step to arrive at the equation of motion (4.11).

The elements of the frequency matrix, $\Omega$, are again easy to calculate:

$$
\Omega_{\rho j}^{s}=\left\langle\left\langle\rho_{q}^{s} \mid \mathcal{L}_{+} j_{q}^{s L}\right\rangle=q \frac{T}{m},\right.
$$

see appendix F.1 and

$$
\Omega_{j \rho}^{s}=\left\langle\left\langle j_{q}^{s L} \mid \mathcal{L}_{+} \rho_{q}^{s}\right\rangle\right\rangle=q \frac{T}{m},
$$

which follows directly from $\left\langle\left\langle j_{q}^{s L} \mid \mathcal{L}_{+} \rho_{q}^{s}\right\rangle=q\left\langle\left\langle j_{q}^{s L} \mid j_{q}^{s L}\right\rangle\right\rangle\right.$ and

$$
\Omega_{j j}^{s}=\left\langle\left\langle j_{q}^{s L} \mid \mathcal{L}_{+} j_{q}^{s L}\right\rangle\right\rangle=-i \omega_{E} \frac{1+\epsilon}{2}\left[1-j_{0}(2 a q)+2 j_{2}(2 a q)\right]
$$

which is already known from the coherent scattering function.

In order to calculate the memory kernel, I resort to a mode coupling approximation again. Close to the glass transition, the dynamics of the tagged particle will be completely dominated by the slow relaxation of the collective density modes. Therefore, I will use the same mode coupling projector I already introduced for the VACF (see section 5.2,

$$
\left.\mathcal{P}_{2}^{s}=N \sum_{k, p}\left|\rho_{k} \rho_{p}^{s}\right\rangle\right\rangle\left\langle\rho_{k} \rho_{p}^{s}\right| / S_{k}
$$

With the usual set of mode coupling approximations, one gets the following approximation of the memory kernel,

$$
M_{j j}^{s}(q, t) \approx N \sum_{k, p}\left\langle\left\langle j_{q}^{s L} \mid \mathcal{L}_{+} \mathcal{Q}_{k} \rho_{p}^{s}\right\rangle\right\rangle\left\langle\left\langle\rho_{k} \rho_{p}^{s} \mathcal{Q} \mid \mathcal{L}_{+} j_{q}^{s L}\right\rangle\right\rangle \phi(k, t) \phi^{s}(p, t) / S_{k},
$$

which is the wave number dependent generalization of the memory kernel $M_{v \rho}(t)$, eq. (5.18).

The left vertex is easily calculated (see appendix F.2.2) and it is again identical to the one for elastic hard spheres,

$$
V\left\langle\left\langle j_{q}^{s L} \mid \mathcal{Q} \mathcal{L}_{+} \rho_{k} \rho_{p}^{s}\right\rangle\right\rangle=\frac{1}{N} \frac{T}{m}(\hat{\boldsymbol{q}} \cdot \boldsymbol{k})\left(S_{k}-1\right) \delta(\boldsymbol{q}-\boldsymbol{k}-\boldsymbol{p}),
$$

while the right vertex is decorated with an $\epsilon$-dependent prefactor again,

$$
V\left\langle\left\langle\rho_{k} \rho_{p}^{s} \mathcal{Q} \mid \mathcal{L}_{+} j_{q}^{s L}\right\rangle=\frac{1+\epsilon}{2} \frac{1}{N} \frac{T}{m}(\hat{\boldsymbol{q}} \cdot \boldsymbol{k})\left(S_{k}-1\right) \delta(\boldsymbol{q}-\boldsymbol{k}-\boldsymbol{p}) .\right.
$$

This leads to a memory kernel

$$
M_{j j}^{s}(q, t)=\frac{1+\epsilon}{2} \frac{T}{m} \frac{n^{2}}{N} \sum_{\boldsymbol{k}+\boldsymbol{p}=\boldsymbol{q}} S_{k}(\hat{\boldsymbol{q}} \cdot \boldsymbol{k})^{2} c_{k}^{2} \phi(k, t) \phi_{s}(p, t),
$$



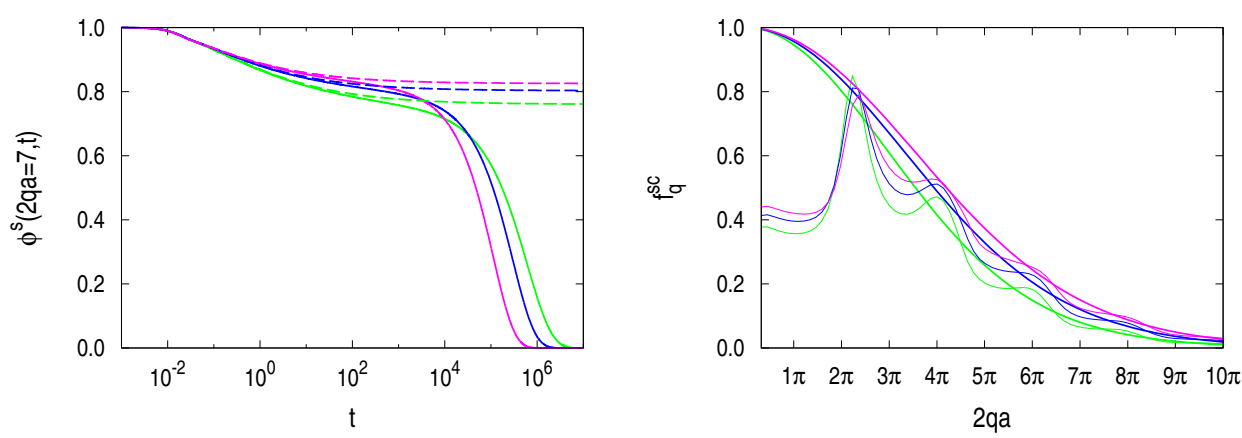

Figure 7.1: Left: The incoherent scattering function, $\phi^{s}(q, t)$, as a function of time for a characteristic wave number, $2 q a=7.0$, several values of the coefficient of restitution $\epsilon=1.0$ (green), 0.5 (blue), 0.0 (magenta) at the critical density $\varphi_{c}$ (dashed lines) and close to the glass transition, $\Delta \varphi(\epsilon)=10^{-3}$ (solid lines). Right: Critical Lamb-Mößbauer factor, $f_{q}^{s c}$ (thick lines), and EA order parameter, $f_{q}^{c}$ (thin lines; see Fig. 6.2 b), for several values of the coefficient of restitution $\epsilon=1.0$ (green), 0.5 (blue), 0.0 (magenta).

or, in dimensionless form,

$$
m^{s}\left[\phi, \phi^{s}\right](q, t)=-\frac{1+\epsilon}{2} \frac{n}{q^{2}} \int d^{3} k S_{k}(\hat{\boldsymbol{q}} \cdot \boldsymbol{k})^{2} c_{k}^{2} \phi(k, t) \phi_{s}(\boldsymbol{q}-\boldsymbol{k}, t) .
$$

Again, the memory kernel acquired a prefactor that depends on the coefficient of restitution but that is strictly positive. The memory kernel for the mean square displacement, $m_{0}(t)=\lim _{q \rightarrow 0} q^{2} m^{s}(q, t)$, is identical to the memory kernel $M_{v \rho}(t)$ [eq. [5.18)]

\subsection{Discussion}

The mode coupling approximation of the memory kernel $m^{s}(q, t)$ transformed the equation of motion (4.11) into closed equation for the incoherent scattering function, $\phi^{s}(q, t)$, once the coherent scattering function, $\phi(q, t)$, and the static structure factor, $S_{q}$, are specified. Using the coherent scattering function in the mode coupling approximation, one expects that the corresponding solution for the incoherent scattering function, $\phi^{s}(q, t)$ is valid close to the glass transition. Fig. 7.1 shows the incoherent scattering function for a number of values of the coefficient of restitution $\epsilon$ at volume fractions $\Delta \varphi(\epsilon)=10^{-3}$ close to the critical density, $\varphi_{c}(\epsilon)$. The latter also depends on the coefficient of restitution as I have shown in the previous chapter. The two step relaxation scenario is also observed for the incoherent scattering function. The plateau value, $f_{q}^{s c}$, (see Fig. 7.1) is structurally much simpler than the EA order parameter for the coherent scattering function which is also shown for comparison. 

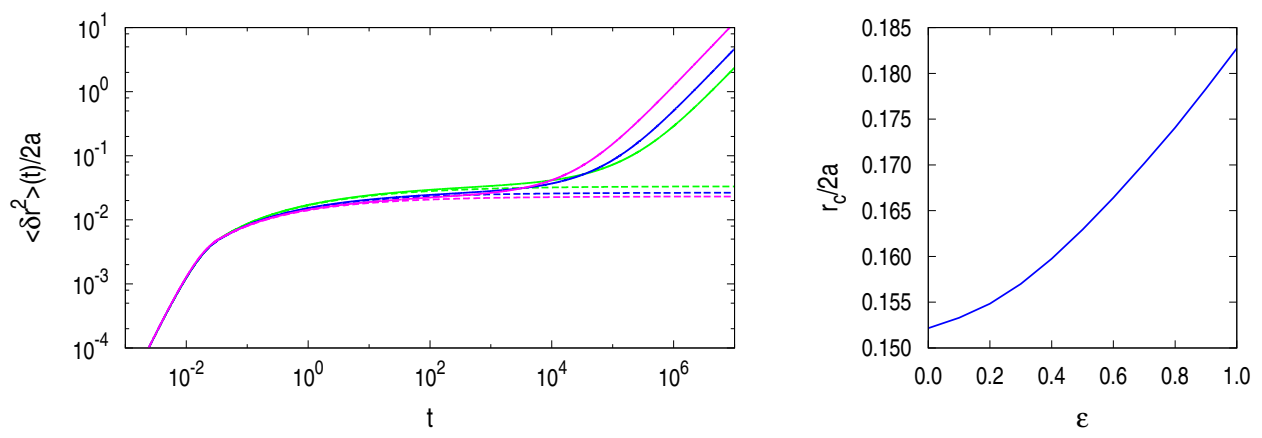

Figure 7.2: Left: Mean square displacement, $\left\langle\delta r^{2}\right\rangle(t)$, as a function of time at the critical density, $\varphi_{c}(\epsilon)$ (dashed lines), and close to the glass transition $\left[\Delta \varphi(\epsilon)=10^{-3}\right]$ (solid lines) for some values of the coefficient of restitution $\epsilon=1.0$ (green), 0.5 (blue), 0.0 (magenta). Right: Localization length, $r_{c}$, as a function of the coefficient of restitution, $\epsilon$.

Consistent with the result for the EA order parameter, the persistent correlations, $f_{q}^{s c}$, are stronger for the more inelastic systems.

The mean square displacement, $\left\langle\delta r^{2}\right\rangle(t)$, is shown in Fig. 7.2. Comparison with the simulation results in Fig. 1.8 for a two dimensional system, and indeed with all the mean square displacements in that figure show that the granular mode coupling theory strongly supports a localization transition of the driven granular fluid. The critical localization length, $r_{c}(\epsilon)$, as a function of the coefficient of restitution, $\epsilon$ (Fig. 7.2 shows that the more inelastic the particles are, the more tightly localized they are at the granular glass transition.

While the localization length is smaller for the more dissipative systems and consequently the plateau of the mean square displacement close to the glass transition is lower, the long time diffusion coefficient, $D_{\infty}$, is larger for inelastic hard spheres compared to a fluid of elastic hard spheres in thermal equilibrium. This is consistent with the simulation results of Fiege et al. [67].

To allow for a quantitative comparison with the experimental and numerical results, the granular mode coupling theory developed here has to be formulated for twodimensional systems. In addition, the numerically exact static structure factors from simulations should be used instead of the Percus-Yevick approximation for elastic hard spheres.

Again, it would of course be interesting to apply the present formalism to other nonequilibrium systems. I expect that for mesoscopic systems, where it may always be challenging to obtain a dynamic scattering function experimentally, the mean square displacement will be an accessible observable to detect a glass transition. 


\section{A The Laplace Transform}

I use the following definitions for the Fourier transform, $\psi^{\prime \prime}(\omega)=\mathrm{FT}[\psi](\omega)$, its inverse and the Laplace transform, $\hat{\psi}(s)=\operatorname{LT}[\psi](s)$,

$$
\begin{aligned}
\psi^{\prime \prime}(\omega) & :=\frac{1}{2} \int_{-\infty}^{\infty} d t e^{-i \omega t} \psi(t) \\
\psi(t) & =\frac{1}{\pi} \int_{-\infty}^{\infty} d \omega e^{i \omega t} \psi^{\prime \prime}(\omega) \\
\hat{\psi}(s) & :=i \int_{0}^{\infty} d t e^{-i s t} \psi(t) .
\end{aligned}
$$

With these definitions the following relation between the Laplace transform $\hat{\psi}(s)$ and the Fourier transform $\psi^{\prime \prime}(\omega)$ holds,

$$
\begin{aligned}
\hat{\psi}(s) & =\frac{i}{\pi} \int_{0}^{\infty} d t e^{-i s t} \int_{-\infty}^{\infty} d \omega e^{i \omega t} \psi^{\prime \prime}(\omega) \\
& =\frac{i}{\pi} \int_{-\infty}^{\infty} d \omega \psi^{\prime \prime}(\omega) \int_{0}^{\infty} d t e^{-i s t} e^{i \omega t} \\
& =\frac{1}{\pi} \int_{-\infty}^{\infty} d \omega \frac{\psi^{\prime \prime}(\omega)}{s-\omega} .
\end{aligned}
$$

With the following famous identity for integral kernels,

$$
\lim _{\epsilon \rightarrow 0} \frac{1}{\omega-i \epsilon}=\mathrm{P} \frac{1}{\omega}+i \pi \delta(\omega)
$$

one can show that

$$
\begin{aligned}
\hat{\psi}(\omega) & =\lim _{\epsilon \rightarrow 0} \hat{\psi}(\omega-i \epsilon)=\mathrm{P} \int_{-\infty}^{\infty} \frac{d \Omega}{\pi} \frac{\psi^{\prime \prime}(\Omega)}{\omega-\Omega}+i \psi^{\prime \prime}(\omega) \\
& =\psi^{\prime}(\omega)+i \psi^{\prime \prime}(\omega)
\end{aligned}
$$

i.e. the Fourier transform, $\psi^{\prime \prime}(\omega)$, is just the imaginary part of $\hat{\psi}(\omega)$. This in turn is connected to the real part via the Cramers-Kronig relation,

$$
\psi^{\prime}(\omega)=\mathrm{P} \int_{-\infty}^{\infty} \frac{d \Omega}{\pi} \frac{\psi^{\prime \prime}(\Omega)}{\omega-\Omega}
$$





\section{B Driving}

For simplicity I concentrate on a one particle system that is only affected by the driving. The generalization to more particles and additional forces is straight forward. In the case of kicks that happen with a finite frequency $f_{D}=1 / \tau_{D}$ and with a characteristic strength, $v_{D}$, the corresponding Liouville operator reads

$$
i \mathcal{L}_{D}^{+} A(\boldsymbol{v} ; t)=\sum_{n}\left[A\left(\boldsymbol{v}+v_{D} \boldsymbol{\xi}_{n} ; t\right)-A(\boldsymbol{v} ; t)\right] \delta\left(t-n \tau_{D}\right)
$$

where the $\boldsymbol{\xi}_{n}$ are random gaussian variables with zero mean $\left\langle\boldsymbol{\xi}_{n}\right\rangle=0$ and variance $\left\langle\xi_{n}^{\alpha} \xi_{m}^{\beta}\right\rangle=\delta^{\alpha \beta} \delta_{n m}$.

Due to the $\delta$-function in equation (B.1), one should look at the change due to driving integrated over a small time interval. Doing this, one finds for the change in momentum $\boldsymbol{p}=m \boldsymbol{v}$ and energy $E=m \boldsymbol{v}^{2} / 2$,

$$
\frac{1}{\tau_{D}} \int^{*} d t i \mathcal{L}_{D} \boldsymbol{p}(t)=m v_{D} \boldsymbol{\xi}_{n} / \tau_{D}
$$

where $\int^{*} d t$ denotes the time integral over one single kick;

$$
\frac{1}{\tau_{D}} \int^{*} d t i \mathcal{L}_{D} E(t)=m \frac{v_{D}}{\tau_{D}} \boldsymbol{v}(t) \cdot \boldsymbol{\xi}_{n}+\frac{m v_{D}^{2}}{2 \tau_{D}} \boldsymbol{\xi}_{n}^{2} .
$$

Lets define $i \tilde{\mathcal{L}}_{D}^{+} A:=\tau_{D}^{-1} \int^{*} d t i \mathcal{L}_{D} A$. Then we get $\left\langle i \tilde{\mathcal{L}}_{D}^{+} \boldsymbol{p}\right\rangle=0$ and $\left\langle i \tilde{\mathcal{L}}_{D}^{+} E\right\rangle=$ $m v_{D}^{2} / 2 \tau_{D} \equiv P_{D}$ which defines the driving power, $P_{D}$. In the end, the driving power that will compensate the collisional cooling is all we care about. Therefore it is best to think of the driving as being defined by $P_{D}$ and $\tau_{D}$ and to regard $v_{D}=\sqrt{2 P_{D} \tau_{D} / m}$ as a derived quantity.

If we consider the result of a finite number $N$ of kicks, we find that due to the $\boldsymbol{\xi}_{n}$ being gaussian random variables, the total effect can be written in the form

$$
\int_{0}^{t} d t^{\prime} i \mathcal{L}_{D} A\left(\boldsymbol{v} ; t^{\prime}\right)=A(\boldsymbol{v}(0)+\Delta \boldsymbol{v}(t))-A(\boldsymbol{v} ; 0),
$$

where $t=N \tau_{D}$ and, $\Delta \boldsymbol{v}(t) \sim \mathcal{N}\left(0,2 N P_{D} \tau_{D} / m\right)=\mathcal{N}\left(0,2 P_{D} t / m\right)$, is again a gaussian random variable. Assuming we let $\tau_{D} \rightarrow 0$ this defines a Wiener process for the velocity $\boldsymbol{v}(t)$. The corresponding stochastic differential equation reads

$$
\frac{d \boldsymbol{v}}{d t}=\sqrt{2 P_{D} / m} \boldsymbol{\xi}(t)
$$


where the $\boldsymbol{\xi}(t)$ are now gaussian random variables with zero mean, $\langle\boldsymbol{\xi}(t)\rangle=0$, and variance $\left\langle\xi^{\alpha}(t) \xi^{\beta}\left(t^{\prime}\right)\right\rangle=\delta^{\alpha \beta} \delta\left(t-t^{\prime}\right)$. According to Ito calculus 1 we then have for an observable $A$,

$$
\begin{aligned}
d A(\boldsymbol{v}) & =A^{\prime}(\boldsymbol{v}) d v+\frac{1}{2} A^{\prime \prime}(\boldsymbol{v}) d v^{2}+\mathcal{O}\left(d v^{3}\right) \\
& =A^{\prime}(\boldsymbol{v}) d v+A^{\prime \prime}(\boldsymbol{v}) \frac{P_{D}}{m} d t
\end{aligned}
$$

where $A^{\prime}(\boldsymbol{v}) \equiv \partial A / \partial \boldsymbol{v}$. Keeping in mind that the $\boldsymbol{\xi}(t)$ have awkward properties and should in fact always be integrated over time, we can, nevertheless, define a corresponding Liouville operator

$$
i \mathcal{L}_{D}^{\infty}=\sqrt{2 P_{D} / m} \boldsymbol{\xi}(t) \cdot \frac{\partial}{\partial \boldsymbol{v}}+\frac{P_{D}}{m} \frac{\partial^{2}}{\partial \boldsymbol{v}^{2}} .
$$

One can check that it has the right properties, i.e., $\left\langle i \mathcal{L}_{D}^{\infty} \boldsymbol{p}\right\rangle=\sqrt{2 P_{D} m}\langle\boldsymbol{\xi}\rangle=0$ and $\left\langle i \mathcal{L}_{D}^{\infty} E\right\rangle=\sqrt{P_{D} m / 2}\langle\boldsymbol{\xi} \cdot \boldsymbol{v}\rangle+P_{D}=P_{D}$.

Alternatively, this limiting behavior for $\tau_{D} \rightarrow 0$ can also be derived specifically from equation (B.1). The expression $i \tilde{\mathcal{L}}_{D} A(\boldsymbol{v})$ can be expanded as

$$
\begin{aligned}
i \tilde{\mathcal{L}}_{D} A\left(v^{\alpha}\right) & =\frac{A\left(v^{\alpha}+C \sqrt{\tau_{D}} \xi_{n}^{\alpha}\right)-2 A\left(v^{\alpha}+C \sqrt{\tau_{D}} \xi_{n}^{\alpha} / 2\right)+A\left(v^{\alpha}\right)}{\tau_{D}} \\
& +\frac{2 A\left(v^{\alpha}+C \sqrt{\tau_{D}} \xi_{n}^{\alpha} / 2\right)-2 A\left(v^{\alpha}\right)}{\tau_{D}}
\end{aligned}
$$

where $C:=\sqrt{2 P_{D} / m}$. In the continuous limit this yields,

$$
\lim _{\tau_{D} \rightarrow 0} i \tilde{\mathcal{L}}_{D} A(\boldsymbol{v})=A^{\prime}(\boldsymbol{v}) \lim _{\tau_{D} \rightarrow 0} \sqrt{2 P_{D} / m \tau_{D}} \boldsymbol{\xi}_{n}+A^{\prime \prime}(\boldsymbol{v}) \frac{P_{D}}{2 m} \boldsymbol{\xi}_{n}^{2}
$$

which turns into equation (B.7) when we make the replacements $\lim _{\tau_{D} \rightarrow 0} \boldsymbol{\xi}_{n} / \sqrt{\tau_{D}} \rightarrow$ $\boldsymbol{\xi}(t)$, which underlines that the $\boldsymbol{\xi}(t)$ are almost surely infinite, and $\lim _{\tau_{D} \rightarrow 0}\left\langle\xi_{n}^{\alpha} \xi_{m}^{\beta}\right\rangle \rightarrow$ $2\left\langle\xi^{\alpha}(t) \xi^{\beta}\left(t^{\prime}\right)\right\rangle$.

\footnotetext{
${ }^{1}$ We need the relations $d v^{2}=d t$ and $d v^{M}=0$ for $M>2$.
} 


\section{Fluctuating Hydrodynamics}

\section{C.1 Hydrodynamic Eigenvalues}

The eigen values are determined by

$$
\begin{aligned}
\operatorname{det}[\mathrm{M}(k)-i \omega]= & \left|\begin{array}{ccc}
i \omega & 0 & i k n \\
g \Gamma / n & D_{T} k^{2}+3 \Gamma / 2 T+i \omega & 2 i k p / 3 n \\
i k c_{T}^{2} / n & i k p / m n T & \nu_{\ell} k^{2}+i \omega
\end{array}\right| \\
= & i \omega\left[\left(D_{T} k^{2}+3 \Gamma / 2 T+i \omega\right)\left(\nu_{\ell} k^{2}+i \omega\right)+2 k^{2} p^{2} / 3 m n^{2} T\right] \\
& -k^{2}\left[g p \Gamma / m n T-c_{T}^{2}\left(D_{T} k^{2}+3 \Gamma / 2 T+i \omega\right)\right] \\
\simeq & -i \omega^{3}-\omega^{2}\left[\left(D_{T}+\nu_{l}\right) k^{2}+3 \Gamma / 2 T\right] \\
& +i \omega k^{2}\left(c_{T}^{2}+2 p^{2} / 3 m n^{2} T+3 \Gamma \nu_{\ell} / 2 T\right) \\
& -g p \Gamma k^{2} / m n T+c_{T}^{2} k^{2}\left(D_{T} k^{2}+3 \Gamma / 2 T\right)=0 .
\end{aligned}
$$

The isothermal speed of sound, $c_{T}^{2}=\frac{\partial p}{\partial m n} \simeq T / m$, is equal to the thermal velocity in a dilute gas. With the molecular chaos assumption, the cooling rate, $\Gamma / T \approx$ $\frac{1-\epsilon^{2}}{3} \omega_{0}$, is always smaller than the collision frequency $\omega_{c}$. Again in the dilute limit, $D_{T} \simeq \nu_{\ell}=\mathcal{O}\left(\ell_{0}^{2} \omega_{c}\right)$. Finally, we have $\ell_{0}^{2} \omega_{c}^{2}=T / m$. For the mixed term in the above equation these relations imply $3 \Gamma \nu_{\ell} / 2 T \simeq\left(1-\epsilon^{2}\right) T / m$.

In the limit $\left(D_{T}+\nu_{\ell}\right) k^{2} \ll 3 \Gamma / 2 T$ we have

$$
-i \omega^{3}-\frac{3 \Gamma}{2 T} \omega^{2}+i \omega k^{2}\left(c_{T}^{2}+2 p^{2} / 3 m n^{2} T+3 \Gamma \nu_{\ell} / 2 T\right)+\frac{3 \Gamma}{2 T} k^{2}\left(c_{T}^{2}-2 g p / 3 m n\right)=0 .
$$

With the ansatz

$$
\begin{aligned}
(i \omega+3 \Gamma / 2 T)\left(i \omega+i c_{<}\right. & \left.k+D_{S}^{<} k^{2}\right)\left(i \omega-i c_{<} k+D_{S}^{<} k^{2}\right) \\
& \simeq-i \omega^{3}-\frac{3 \Gamma}{2 T} \omega^{2}+i \omega k^{2}\left(c_{<}^{2}+3 \Gamma D_{S}^{<} / T\right)+\frac{3 \Gamma}{2 T} c_{<}^{2} k^{2},
\end{aligned}
$$

we get

$$
\begin{gathered}
c_{<}^{2}=c_{T}^{2}-2 g p / 3 m n, \\
2 D_{S}^{<}=\nu_{\ell}+\frac{4 p T}{9 m n \Gamma}(g+p / n T) .
\end{gathered}
$$




\section{C.2 Structure Factors from Fluctuating Hydrodynamics}

Formally integrating eq. $(3.23)$ we get

$$
\begin{aligned}
\delta \boldsymbol{a}(t) & =e^{t M} \delta \boldsymbol{a}(0)+\int_{0}^{t} d \tau e^{(t-\tau) M} \boldsymbol{f}(\tau) \\
& =\mathrm{U}(t) \delta \boldsymbol{a}(0)+\int_{0}^{t} d \tau \mathrm{U}(t-\tau) \boldsymbol{f}(\tau),
\end{aligned}
$$

which defines the propagator U. Multiplying the above equation by $\delta \boldsymbol{a}^{*}(t)$ and taking the average, we get

$$
\left\langle\delta a_{a}(t) \delta a_{b}^{*}(t)\right\rangle=\mathrm{S}_{a b}=\mathrm{U}_{a c}(t) \mathrm{U}_{b d}^{*}(t) \mathrm{S}_{c d}+\int_{0}^{t} d \tau \mathrm{U}_{a c}(\tau) \mathrm{U}_{b d}^{*}(\tau) \Gamma_{c d} .
$$

Taking the time derivative of $\mathrm{S}$ we get

$$
0=\mathrm{M}_{f c}(\boldsymbol{k}) \mathrm{U}_{a f}(t) \mathrm{U}_{b d}^{*}(t) \mathrm{S}_{c d}(\boldsymbol{k})+\mathrm{U}_{a c}(t) \mathrm{M}_{f d}(-\boldsymbol{k}) \mathrm{U}_{b f}^{*}(t) \mathrm{S}_{c d}(\boldsymbol{k})+\mathrm{U}_{a c}(t) \mathrm{U}_{b d}^{*}(t) \Gamma_{c d}(\boldsymbol{k}) .
$$

Setting the time to zero all the propagators degenrate to identies and we get

$$
0=\mathrm{M}_{a c}(\boldsymbol{k}) \mathrm{S}_{c b}(\boldsymbol{k})+\mathrm{M}_{b d}(-\boldsymbol{k}) \mathrm{S}_{a d}(\boldsymbol{k})+\Gamma_{a b}(\boldsymbol{k}),
$$

or

$$
-\mathrm{M}(\boldsymbol{k}) \mathrm{S}(\boldsymbol{k})-\mathrm{S}(\boldsymbol{k}) \mathrm{M}^{T}(-\boldsymbol{k})=\Gamma(\boldsymbol{k}) .
$$

Multiplying the above equation with partitions of unity from the left and from the right and invoking the eigenvalue equation we get

$$
\sum_{\lambda, \mu}\left|w_{\lambda}(\boldsymbol{k})\right\rangle\left\langle v_{\lambda}(\boldsymbol{k})|\mathrm{S}(\boldsymbol{k})| v_{\mu}(\boldsymbol{k})\right\rangle\left\langle w_{\mu}(\boldsymbol{k})\right|=-\sum_{\lambda, \mu} \frac{\left|w_{\lambda}(\boldsymbol{k})\right\rangle\left\langle v_{\lambda}(\boldsymbol{k})|\Gamma(\boldsymbol{k})| v_{\mu}(\boldsymbol{k})\right\rangle\left\langle w_{\mu}(\boldsymbol{k})\right|}{z_{\lambda}(k)+z_{\mu}^{*}(k)} .
$$

Upon rewriting the terms a little we get eq. (3.25). 


\section{Mode Coupling Diagrams}

In this chapter I want to give a few more details on the diagrams used in chapter 4. While the interpretation is physically most transparent in the time domain, the formulas become a little easier in the Laplace domain. In particular the convolution theorem, $\int_{0}^{\infty} f(\tau) g(t-\tau) d \tau \rightarrow \hat{f}(s) \hat{g}(s)$, reduces the number of integrals involved.

Following Wyld [323], I introduce a formal four-vector $\check{k}:=(s, \boldsymbol{k})$. For simplicity, I further assume that the vertices $\mathcal{V}_{q k p}$ are independent of wave number except for

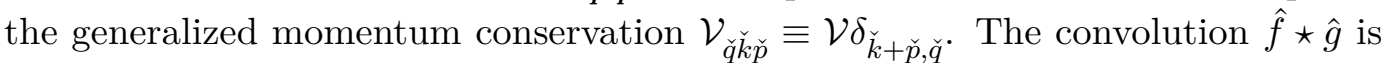
defined as $(\hat{f} \star \hat{g})(\check{k})=\int d^{4} p \hat{f}(\check{p}) \hat{g}(\check{k}-\check{p})=\int d^{3} p \int_{0}^{s} d \sigma \hat{f}(\sigma, \boldsymbol{p}) \hat{g}(s-\sigma, \boldsymbol{k}-\boldsymbol{p})$.

The equation of motion for the VACF reads in the Laplace domain,

$$
\hat{\psi}(s)=\hat{\psi}_{0}(s)+\hat{\psi}_{0}(s) \hat{M}_{v}(s) \hat{\psi}(s),
$$

and with the mode coupling approximation, $\hat{M}_{v}(s) \approx \hat{M}_{v \rho}=\mathcal{V}^{2}\left(\hat{\phi} \star \hat{\phi}^{s}\right)(s, \boldsymbol{q}=\mathbf{0})$, we have

$$
\hat{\psi}(s)=\hat{\psi}_{0}(s)+\hat{\psi}_{0}(s) \mathcal{V}^{2}\left(\hat{\phi} \star \hat{\phi}^{s}\right)(s, \mathbf{0}) \hat{\psi}(s) .
$$

For the second order equation of motion for the coherent scattering function $\phi(q, t)$ one finds

$$
\hat{\phi}(\check{q})=\hat{\phi}_{0}(\check{q})+\hat{\phi}_{0}(\check{q}) \hat{M}(\check{q})[\hat{\phi}(\check{q})-1 / s],
$$

i.e, with $\hat{M}(\check{q}) \approx \mathcal{V}^{2}(\hat{\phi} \star \hat{\phi})(\check{q})$,

$$
\hat{\phi}(\check{q})=\hat{\phi}_{0}(\check{q})+\hat{\phi}_{0}(\check{q}) \mathcal{V}^{2}(\hat{\phi} \star \hat{\phi})(\check{q})[\hat{\phi}(\check{q})-1 / s] .
$$

With the following three identifications and the additional rule that there is an integration $\int d^{4} k_{i}$ over every internal variable $\breve{k}_{i}$ both eq. (D.2) and eq. (D.4) can be represented by diagrams that are structurally identical:

$$
\begin{aligned}
\overline{\phi_{0}} & =\hat{\phi}_{0}(\check{q}) \\
\bar{\phi} & =\hat{\phi}(\check{q}) \\
-\quad & =\mathcal{V} \delta_{\check{k}+\check{p}, \check{q}} .
\end{aligned}
$$





\section{E The Mori Identity}

Starting from the trivial identity

$$
(s+\mathcal{L})(s+\mathcal{L})^{-1}=1
$$

insert $1=\mathcal{P}+\mathcal{Q}$

$$
(s+\mathcal{L}) \mathcal{P}(s+\mathcal{L})^{-1}+(s+\mathcal{L}) \mathcal{Q}(s+\mathcal{L})^{-1}=1
$$

use $\mathcal{P}^{2}=\mathcal{P}$

$$
(s \mathcal{P}+\mathcal{P} \mathcal{L}) \mathcal{P}(s+\mathcal{L})^{-1}+(s \mathcal{P}+\mathcal{P} \mathcal{L}) \mathcal{Q}(s+\mathcal{L})^{-1}=\mathcal{P}
$$

and $\mathcal{P} \mathcal{Q}=0$ and do the same again with $\mathcal{Q}$

$$
\begin{aligned}
& (s+\mathcal{P} \mathcal{L P}) \mathcal{P}(s+\mathcal{L})^{-1}+\mathcal{P} \mathcal{L} \mathcal{Q}(s+\mathcal{L})^{-1}=P \\
& (s+\mathcal{Q} \mathcal{L} \mathcal{Q}) \mathcal{Q}(s+\mathcal{L})^{-1}+\mathcal{Q} \mathcal{L} \mathcal{P}(s+\mathcal{L})^{-1}=Q
\end{aligned}
$$

Multiply with $\mathcal{P}$ from the right

$$
\begin{gathered}
(s+\mathcal{P} \mathcal{L} \mathcal{P}) \mathcal{P}(s+\mathcal{L})^{-1} \mathcal{P}+(\mathcal{P} \mathcal{L} \mathcal{Q}) \mathcal{Q}(s+\mathcal{L})^{-1} \mathcal{P}=\mathcal{P}^{2}=\mathcal{P} \\
(s+\mathcal{Q} \mathcal{L} \mathcal{Q}) \mathcal{Q}(s+\mathcal{L})^{-1} \mathcal{P}+(\mathcal{Q L} \mathcal{P}) \mathcal{P}(s+\mathcal{L})^{-1} \mathcal{P}=0 \\
-(\mathcal{Q} \mathcal{P}) \mathcal{P}(s+\mathcal{L})^{-1} \mathcal{P}=(s+\mathcal{Q} \mathcal{L} \mathcal{Q}) \mathcal{Q}(s+\mathcal{L})^{-1} \mathcal{P}
\end{gathered}
$$

Substitute this in the second equation

$$
\left[s+\mathcal{P} \mathcal{L} \mathcal{P}-\mathcal{P} \mathcal{L} Q(s+\mathcal{Q} \mathcal{L})^{-1} \mathcal{Q L} \mathcal{P}\right] \mathcal{P}(s+\mathcal{L})^{-1} \mathcal{P}=\mathcal{P}
$$





\section{F Matrix Elements of the Liouville Operator}

In this appendix you can find the detailed calculation of the matrix elements of the Liouville operator as they are needed in the main part of the text. Purely technical aspects of the calculation that are used repeatedly are collected in section F.3.

Note the following important identities

$$
\begin{gathered}
i \mathcal{L}_{0} j_{q}^{L}=\frac{1}{N} \frac{i}{q} \sum_{l}\left(\boldsymbol{q} \cdot \boldsymbol{v}_{l}\right)^{2} e^{i \boldsymbol{q} \cdot \boldsymbol{r}_{i}} \\
i \mathcal{T}_{12}^{+} j_{q}^{L}=-\frac{1+\epsilon}{2 N}\left(\hat{\boldsymbol{r}}_{12} \cdot \boldsymbol{v}_{12}\right)^{2}\left(\hat{\boldsymbol{q}} \cdot \hat{\boldsymbol{r}}_{12}\right) \Theta\left(-\hat{\boldsymbol{r}}_{12} \cdot \boldsymbol{v}_{12}\right) \delta\left(r_{12}-2 a\right)\left(e^{i \boldsymbol{q} \cdot \boldsymbol{r}_{2}}-e^{i \boldsymbol{q} \cdot \boldsymbol{r}_{1}}\right), \\
i \mathcal{T}_{1 s}^{+} j_{q}^{s L}=-\frac{1+\epsilon}{2}\left(\hat{\boldsymbol{r}}_{1 s} \cdot \boldsymbol{v}_{1 s}\right)^{2}\left(\hat{\boldsymbol{q}} \cdot \hat{\boldsymbol{r}}_{1 s}\right) \Theta\left(-\hat{\boldsymbol{r}}_{1 s} \cdot \boldsymbol{v}_{1 s}\right) \delta\left(r_{1 s}-2 a\right) e^{i \boldsymbol{q} \cdot \boldsymbol{r}_{s}}
\end{gathered}
$$

As velocities appear at most linearly in all of the following matrix elements, there is no explicit contribution of the driving operator, $\mathcal{L}_{D}^{+}$.

\section{F.1 The Frequency Matrix}

For the tagged particle quantities one finds,

$$
\left\langle\left\langle\rho_{q}^{s} \mid \mathcal{L}_{+} \rho_{q}^{s}\right\rangle\right\rangle=\left\langle\rho_{q}^{s} \mid \mathcal{L}_{0} \rho_{q}^{s}\right\rangle=q\left\langle\rho_{q}^{s} \mid j_{q}^{s L}\right\rangle=0,
$$

due to parity and,

$$
\left\langle\left\langle j_{q}^{s L} \mid \mathcal{L}_{+} \rho_{q}^{s}\right\rangle\right\rangle=q\left\langle j_{q}^{s L} \mid j_{q}^{s L}\right\rangle=q \frac{T}{m},
$$

in agreement with known results [115].

The element,

$$
\left\langle\left\langle\rho_{q}^{s} \mid \mathcal{L}_{+} j_{q}^{s L}\right\rangle=\left\langle\rho_{q}^{s} \mid \mathcal{L}_{0} j_{q}^{s L}\right\rangle+\left\langle\rho_{q}^{s} \mid \mathcal{T}_{+} j_{q}^{s L}\right\rangle,\right.
$$

is more difficult to determine. First, one finds,

$$
\left\langle\rho_{q}^{s} \mid \mathcal{L}_{0} j_{q}^{s L}\right\rangle=q\left\langle e^{-i \boldsymbol{q} \cdot \boldsymbol{r}_{s}}\left(\hat{\boldsymbol{q}} \cdot \boldsymbol{v}_{s}\right)^{2} e^{i \boldsymbol{q} \cdot \boldsymbol{r}_{s}}\right\rangle=q \frac{T}{m} .
$$


The collisional contribution,

$$
\left\langle\rho_{q}^{s} \mid \mathcal{T}_{+} j_{q}^{s L}\right\rangle=i \frac{1+\epsilon}{2} N\left\langle\left(\hat{\boldsymbol{r}}_{1 s} \cdot \boldsymbol{v}_{1 s}\right)^{2}\left(\hat{\boldsymbol{q}} \cdot \hat{\boldsymbol{r}}_{1 s}\right) \Theta\left(-\hat{\boldsymbol{r}}_{1 s} \cdot \boldsymbol{v}_{1 s}\right) \delta\left(r_{1 s}-2 a\right)\right\rangle=0,
$$

vanishes because the average $\left\langle\left(\hat{\boldsymbol{q}} \cdot \hat{\boldsymbol{r}}_{1 s}\right)\right\rangle=0$ in an isotropic system. The element $\left\langle\left\langle j_{q}^{s L} \mid \mathcal{L}_{+} j_{q}^{s L}\right\rangle\right.$ will be discussed below [see eq. [F.20 ] $]$.

There is one vanishing element for the coherent modes,

$$
\left\langle\left\langle\rho_{q} \mid \mathcal{L}_{+} \rho_{q}\right\rangle\right\rangle=q\left\langle\rho_{q} \mid j_{q}^{L}\right\rangle=0 .
$$

The known off-diagonal element can be reduced to [115],

$$
\left.\left\langle j_{q}^{L} \mid \mathcal{L}_{+} \rho_{q}\right\rangle\right\rangle=q\left\langle j_{q}^{L} \mid j_{q}^{L}\right\rangle=\frac{q}{N} \frac{T}{m} .
$$

The second off-diagonal element, $\left\langle\left\langle\rho_{q} \mid \mathcal{L}_{+} j_{q}^{L}\right\rangle\right\rangle$, is again more difficult to determine. The free streaming contribution is,

$$
\left\langle\left\langle\rho_{q} \mid \mathcal{L}_{0} j_{q}^{L}\right\rangle\right\rangle=\frac{q}{N^{2}}\left\langle\sum_{i, j} e^{-i \boldsymbol{q} \cdot \boldsymbol{r}_{i}}\left(\hat{\boldsymbol{q}} \cdot \boldsymbol{v}_{j}\right)^{2} e^{i \boldsymbol{q} \cdot \boldsymbol{r}_{j}}\right\rangle .
$$

The velocity integration yields a factor $T / m$ while the remaining spatial integration defines the static structure factor $S_{q}$,

$$
\left\langle\left\langle\rho_{q} \mid \mathcal{L}_{0} j_{q}^{L}\right\rangle=\frac{q}{N} \frac{T}{m} S_{q} .\right.
$$

For the collisional contribution we get the following result,

$$
\begin{aligned}
& \left\langle\rho_{q} \mid \mathcal{T}_{+} j_{q}^{L}\right\rangle= \\
& i \frac{1+\epsilon}{4} \hat{\boldsymbol{q}} \cdot\left\langle\left(\hat{\boldsymbol{r}}_{12} \cdot \boldsymbol{v}_{12}\right)^{2} \hat{\boldsymbol{r}}_{12} \Theta\left(-\hat{\boldsymbol{r}}_{12} \cdot \boldsymbol{v}_{12}\right) \delta\left(r_{12}-2 a\right)\left(e^{i \boldsymbol{q} \cdot \boldsymbol{r}_{2}}-e^{i \boldsymbol{q} \cdot \boldsymbol{r}_{1}}\right) \sum_{i} e^{-i \boldsymbol{q} \cdot \boldsymbol{r}_{i}}\right\rangle .
\end{aligned}
$$

The velocity integration yields a factor $T / 2 m$ while the spatial integration can be expanded into the following types of averages,

$$
\begin{aligned}
\left\langle\hat{\boldsymbol{r}}_{12} \delta\left(r_{12}-2 a\right)\left(e^{i \boldsymbol{q} \cdot \boldsymbol{r}_{2}}-e^{i \boldsymbol{q} \cdot \boldsymbol{r}_{1}}\right) \sum_{i} e^{-i \boldsymbol{q} \cdot \boldsymbol{r}_{i}}\right\rangle & =\left\langle\hat{\boldsymbol{r}}_{12} \delta\left(r_{12}-2 a\right) e^{-i \boldsymbol{q} \cdot \boldsymbol{r}_{1}} e^{i \boldsymbol{q} \cdot \boldsymbol{r}_{2}}\right\rangle \\
& -\left\langle\hat{\boldsymbol{r}}_{12} \delta\left(r_{12}-2 a\right) e^{-i \boldsymbol{q} \cdot \boldsymbol{r}_{2}} e^{i \boldsymbol{q} \cdot \boldsymbol{r}_{1}}\right\rangle \\
& +(N-2)\left\langle\hat{\boldsymbol{r}}_{12} \delta\left(r_{12}-2 a\right) e^{-i \boldsymbol{q} \cdot \boldsymbol{r}_{3}} e^{i \boldsymbol{q} \cdot \boldsymbol{r}_{2}}\right\rangle \\
& -(N-2)\left\langle\hat{\boldsymbol{r}}_{12} \delta\left(r_{12}-2 a\right) e^{-i \boldsymbol{q} \cdot \boldsymbol{r}_{3}} e^{i \boldsymbol{q} \cdot \boldsymbol{r}_{1}}\right\rangle .
\end{aligned}
$$


By relabeling the integration variables, the four terms can be collapsed to two terms,

$$
\begin{aligned}
\left\langle\hat{\boldsymbol{r}}_{12} \delta\left(r_{12}-2 a\right)\left(e^{i \boldsymbol{q} \cdot \boldsymbol{r}_{2}}-e^{i \boldsymbol{q} \cdot \boldsymbol{r}_{1}}\right) \sum_{i} e^{-i \boldsymbol{q} \cdot \boldsymbol{r}_{i}}\right\rangle & =2\left\langle\hat{\boldsymbol{r}}_{12} \delta\left(r_{12}-2 a\right) e^{-i \boldsymbol{q} \cdot \boldsymbol{r}_{1}} e^{i \boldsymbol{q} \cdot \boldsymbol{r}_{2}}\right\rangle \\
& +2(N-2)\left\langle\hat{\boldsymbol{r}}_{12} \delta\left(r_{12}-2 a\right) e^{-i \boldsymbol{q} \cdot \boldsymbol{r}_{3}} e^{i \boldsymbol{q} \cdot \boldsymbol{r}_{2}}\right\rangle .
\end{aligned}
$$

With the help of the YBG-relation the second term can be written as

$$
N\left\langle\left\langle\hat{\boldsymbol{r}}_{12} \delta\left(r_{12}-2 a\right) e^{-i \boldsymbol{q} \cdot \boldsymbol{r}_{3}} e^{i \boldsymbol{q} \cdot \boldsymbol{r}_{2}}\right\rangle=-\frac{1}{N}\left(S_{q}-1\right)-\left\langle\hat{\boldsymbol{r}}_{12} \delta\left(r_{12}-2 a\right) e^{-i \boldsymbol{q} \cdot \boldsymbol{r}_{1}} e^{i \boldsymbol{q} \cdot \boldsymbol{r}_{2}}\right\rangle,\right.
$$

cancelling the first term in equation eq. F.15. We get,

$$
\left\langle\left\langle\rho_{q} \mid \mathcal{T}_{+} j_{q}^{L}\right\rangle\right\rangle=-\frac{1+\epsilon}{2} \frac{q}{N} \frac{T}{m}\left(S_{q}-1\right)
$$

and all in all,

$$
\left\langle\left\langle\rho_{q} \mid \mathcal{L}_{+} j_{q}^{L}\right\rangle=\frac{1}{2} \frac{q}{N} \frac{T}{m}\left[(1-\epsilon) S_{q}+(1+\epsilon)\right] .\right.
$$

The calculation of $\left\langle\left\langle j_{q}^{L} \mid \mathcal{L}_{+} j_{q}^{L}\right\rangle\right\rangle$ is not fundamentally new [278]. The free streaming and driving parts do not contribute and the collisional contribution has already been calculated for elastic hard spheres apart from the usual $\epsilon$-dependent prefactor.

Expanding the different type of contributions,

$$
\begin{aligned}
\left\langle j_{q}^{L} \mid \mathcal{T}_{+} j_{q}^{L}\right\rangle & =i \frac{1+\epsilon}{4}\left\langle\left(\hat{\boldsymbol{q}} \cdot \boldsymbol{v}_{1}\right) e^{-i \boldsymbol{q} \cdot \boldsymbol{r}_{12}}\left(\hat{\boldsymbol{q}} \cdot \hat{\boldsymbol{r}}_{12}\right)\left(\hat{\boldsymbol{r}}_{12} \cdot \boldsymbol{v}_{12}\right)^{2} \Theta\left(-\hat{\boldsymbol{r}}_{12} \cdot \boldsymbol{v}_{12}\right) \delta\left(r_{12}-2 a\right)\right\rangle \\
& -i \frac{1+\epsilon}{4}\left\langle\left(\hat{\boldsymbol{q}} \cdot \boldsymbol{v}_{1}\right)\left(\hat{\boldsymbol{q}} \cdot \hat{\boldsymbol{r}}_{12}\right)\left(\hat{\boldsymbol{r}}_{12} \cdot \boldsymbol{v}_{12}\right)^{2} \Theta\left(-\hat{\boldsymbol{r}}_{12} \cdot \boldsymbol{v}_{12}\right) \delta\left(r_{12}-2 a\right)\right\rangle \\
& +i \frac{1+\epsilon}{4}(N-2)\left\langle\left(\hat{\boldsymbol{r}}_{12} \cdot \boldsymbol{v}_{3}\right) e^{-i \boldsymbol{q} \cdot \boldsymbol{r}_{3}}\left(\hat{\boldsymbol{q}} \cdot \hat{\boldsymbol{r}}_{12}\right)\left(\hat{\boldsymbol{r}}_{12} \cdot \boldsymbol{v}_{12}\right)^{2} \Theta\left(-\hat{\boldsymbol{r}}_{12} \cdot \boldsymbol{v}_{12}\right)\right. \\
& \left.\times \delta\left(r_{12}-2 a\right)\left(e^{i \boldsymbol{q} \cdot \boldsymbol{r}_{2}}-e^{i \boldsymbol{q} \cdot \boldsymbol{r}_{1}}\right)\right\rangle,
\end{aligned}
$$

one finds that the third term vanishes by symmetry while the first term can be expressed in terms of spherical Bessel functions (see section F.3 below) to yield the final result,

$$
\left\langle\left\langle j_{q}^{L} \mid \mathcal{L}_{+} j_{q}^{L}\right\rangle=-i \omega_{E} \frac{1+\epsilon}{2 N} \frac{T}{m}\left[1-j_{0}(2 a q)+2 j_{2}(2 a q)\right] .\right.
$$

The fact that the three particle term does not contribute also implies that the corresponding tagged particle relaxation rate is essentially identical, $\left\langle\left\langle j_{q}^{s L} \mid \mathcal{L}_{+} j_{q}^{s L}\right\rangle=\right.$ $N\left\langle\left\langle j_{q}^{L} \mid \mathcal{L}_{+} j_{q}^{L}\right\rangle\right\rangle$. 


\section{F.2 The Vertices}

\section{F.2.1 The Velocity Autocorrelation Function}

For the coupling to the density mode, the projector $\mathcal{Q}$ does not change the vertices due to parity,

$$
\left\langle\left\langle\boldsymbol{v}_{s} \mid \mathcal{L}_{+} \mathcal{Q}_{k} \rho_{p}^{s}\right\rangle\right\rangle=\left\langle\left\langle\boldsymbol{v}_{s} \mid \mathcal{L}_{+} \rho_{k} \rho_{p}^{s}\right\rangle\right\rangle=\left\langle\boldsymbol{v}_{s} \mid \mathcal{L}_{0} \rho_{k} \rho_{p}^{s}\right\rangle
$$

and

$$
\left\langle\left\langle\rho_{k} \rho_{p}^{s} \mid \mathcal{Q} \mathcal{L}_{+} \boldsymbol{v}_{s}\right\rangle\right\rangle=\left\langle\left\langle\rho_{k} \rho_{p}^{s} \mid \mathcal{L}_{+} \boldsymbol{v}_{s}\right\rangle\right\rangle=\left\langle\rho_{k} \rho_{p}^{s} \mid \mathcal{T}_{+} \boldsymbol{v}_{s}\right\rangle .
$$

This is different for the coupling to the currents,

$$
\left\langle\left\langle v_{s}^{\alpha} \mid \mathcal{L}_{+} \mathcal{Q} j_{k}^{\beta} \rho_{p}^{s}\right\rangle\right\rangle=\left\langle\left\langle v_{s}^{\alpha} \mid \mathcal{L}_{+} j_{k}^{\beta} \rho_{p}^{s}\right\rangle\right\rangle-m\left\langle\left\langle v_{s}^{\alpha} \mid \mathcal{L}_{+} v_{s}^{\alpha}\right\rangle\right\rangle\left\langle v_{s}^{\alpha} \mid j_{k}^{\beta} \rho_{p}^{s}\right\rangle / T
$$

and

$$
\left.\left\langle j_{k}^{\beta} \rho_{p}^{s} \mid \mathcal{Q} \mathcal{L}_{+} v_{s}^{\alpha}\right\rangle\right\rangle=\left\langle\left\langle j_{k}^{\beta} \rho_{p}^{s} \mid \mathcal{L}_{+} v_{s}^{\alpha}\right\rangle\right\rangle-m\left\langle j_{k}^{\beta} \rho_{p}^{s} \mid v_{s}^{\alpha}\right\rangle\left\langle\left\langle v_{s}^{\alpha} \mid \mathcal{L}_{+} v_{s}^{\alpha}\right\rangle / T .\right.
$$

Due to parity one finds

$$
m\left\langle j_{k}^{\beta} \rho_{p}^{s} \mid v_{s}^{\alpha}\right\rangle / T=\frac{\delta(\boldsymbol{k}+\boldsymbol{p})}{N V} \delta^{\alpha \beta} .
$$

The contributions to eq. (F.21) are also of this form,

$$
\left\langle\boldsymbol{v}_{s} \mid \mathcal{L}_{0} \rho_{k} \rho_{p}^{s}\right\rangle=k\left\langle\boldsymbol{v}_{s} \mid j_{k}^{L} \rho_{p}^{s}\right\rangle+p\left\langle\boldsymbol{v}_{s} \mid \rho_{k} j_{p}^{s L}\right\rangle,
$$

where,

$$
p\left\langle\boldsymbol{v}_{s} \mid \rho_{k} j_{p}^{s L}\right\rangle=\frac{p}{N}\left\langle\sum_{j} \boldsymbol{v}_{s} e^{i \boldsymbol{k} \cdot \boldsymbol{r}_{j}}\left(\hat{\boldsymbol{p}} \cdot \boldsymbol{v}_{s}\right) e^{i \boldsymbol{p} \cdot \boldsymbol{r}_{s}}\right\rangle=\frac{T}{m} \frac{\delta(\boldsymbol{k}+\boldsymbol{p})}{N V} \boldsymbol{p} S_{k} .
$$

The three point quantities reduce to

$$
\left\langle\left\langle v_{s}^{\alpha} \mid \mathcal{L}_{+} j_{k}^{\beta} \rho_{p}^{s}\right\rangle\right\rangle=\left\langle v_{s}^{\alpha} \mid \rho_{p}^{s} \mathcal{T}_{+} j_{k}^{\beta}\right\rangle
$$

and

$$
\left\langle\left\langle j_{k}^{\beta} \rho_{p}^{s} \mid \mathcal{L}_{+} v_{s}^{\alpha}\right\rangle\right\rangle=\left\langle j_{k}^{\beta} \rho_{p}^{s} \mid \mathcal{T}_{+} v_{s}^{\alpha}\right\rangle
$$

Explicitly, one finds

$$
\begin{aligned}
\left\langle\rho_{k} \rho_{p}^{s} \mid \mathcal{T}_{+} \boldsymbol{v}_{s}\right\rangle & =-i \frac{1+\epsilon}{2}\left\langle e^{-i \boldsymbol{k} \cdot \boldsymbol{r}_{1}} e^{-i \boldsymbol{p} \cdot \boldsymbol{r}_{s}} \hat{\boldsymbol{r}}_{1 s} \delta\left(r_{1 s}-2 a\right)\left(\hat{\boldsymbol{r}}_{1 s} \cdot \boldsymbol{v}_{1 s}\right)^{2} \Theta\left(-\hat{\boldsymbol{r}}_{1 s} \cdot \boldsymbol{v}_{1 s}\right)\right\rangle \\
& -i \frac{1+\epsilon}{2} N\left\langle e^{-i \boldsymbol{k} \cdot \boldsymbol{r}_{2}} e^{-i \boldsymbol{p} \cdot \boldsymbol{r}_{s}} \hat{\boldsymbol{r}}_{1 s} \delta\left(r_{1 s}-2 a\right)\left(\hat{\boldsymbol{r}}_{1 s} \cdot \boldsymbol{v}_{1 s}\right)^{2} \Theta\left(-\hat{\boldsymbol{r}}_{1 s} \cdot \boldsymbol{v}_{1 s}\right)\right\rangle
\end{aligned}
$$


Upon invoking the YBG theorem on the second term, one finds that the first term is canceled. The velocity integrations can be done immediately,

$$
\left\langle\rho_{k} \rho_{p}^{s} \mid \mathcal{T}_{+} \boldsymbol{v}_{s}\right\rangle=\frac{1+\epsilon}{2} \frac{\delta(\boldsymbol{k}+\boldsymbol{p})}{N V} \frac{T}{m} \hat{\boldsymbol{k}}\left(S_{k}-1\right) .
$$

Moreover,

$$
\left\langle j_{k}^{\beta} \rho_{p}^{s} \mid \mathcal{T}_{+} v_{s}^{\alpha}\right\rangle=\left\langle j_{k}^{\beta} \mid \mathcal{T}_{+} j_{p}^{s, \alpha}\right\rangle=\frac{\delta(\boldsymbol{k}+\boldsymbol{p})}{N V} \delta^{\alpha \beta}\left\langle j_{k}^{s, \alpha} \mid \mathcal{T}_{+} j_{k}^{s, \alpha}\right\rangle,
$$

due to parity. The left vertex is identical.

\section{F.2.2 The Incoherent Scattering Function}

Here we have

$$
\left\langle\left\langle j_{q}^{s L} \mid \mathcal{L}_{+} \mathcal{Q}_{k} \rho_{p}^{s}\right\rangle\right\rangle=\left\langle\left\langle j_{q}^{s L} \mid \mathcal{L}_{+} \rho_{k} \rho_{p}^{s}\right\rangle\right\rangle-\left\langle\left\langle j_{q}^{s L} \mid \mathcal{L}_{+} \rho_{q}^{s}\right\rangle\right\rangle\left\langle\rho_{q}^{s} \mid \rho_{k} \rho_{p}^{s}\right\rangle
$$

and

$$
\left\langle\left\langle\rho_{k} \rho_{p}^{s} \mid \mathcal{Q} \mathcal{L}_{+} j_{q}^{s L}\right\rangle\right\rangle=\left\langle\left\langle\rho_{k} \rho_{p}^{s} \mid \mathcal{L}_{+} j_{q}^{s L}\right\rangle\right\rangle-\left\langle\rho_{k} \rho_{p}^{s} \mid \rho_{q}^{s}\right\rangle\left\langle\left\langle\rho_{q}^{s} \mid \mathcal{L}_{+} j_{q}^{s L}\right\rangle\right\rangle,
$$

where,

$$
\left\langle\rho_{q}^{s} \mid \rho_{k} \rho_{p}^{s}\right\rangle=\frac{1}{N}\left\langle\sum_{j} e^{-i \boldsymbol{q} \cdot \boldsymbol{r}_{s}} e^{i \boldsymbol{k} \cdot \boldsymbol{r}_{j}} e^{i \boldsymbol{p} \cdot \boldsymbol{r}_{s}}\right\rangle=\frac{\delta(\boldsymbol{k}+\boldsymbol{p}-\boldsymbol{q})}{N V} S_{k} .
$$

As usual, the left vertex is easier to determine,

$$
\left\langle\left\langle j_{q}^{s L} \mid \mathcal{L}_{+} \rho_{k} \rho_{p}^{s}\right\rangle\right\rangle=k\left\langle j_{q}^{s L} \mid j_{k}^{L} \rho_{p}^{s}\right\rangle+p\left\langle j_{q}^{s L} \mid \rho_{k} j_{p}^{s L}\right\rangle,
$$

where,

$$
\begin{aligned}
\left\langle j_{q}^{s L} \mid j_{k}^{L} \rho_{p}^{s}\right\rangle & =\frac{1}{N}\left\langle\sum_{j}\left(\hat{\boldsymbol{q}} \cdot \boldsymbol{v}_{s}\right) e^{-i \boldsymbol{q} \cdot \boldsymbol{r}_{s}}\left(\hat{\boldsymbol{k}} \cdot \boldsymbol{v}_{j}\right) e^{i \boldsymbol{k} \cdot \boldsymbol{r}_{j}} e^{i \boldsymbol{p} \cdot \boldsymbol{r}_{s}}\right\rangle \\
& =\frac{T}{m} \frac{\delta(\boldsymbol{k}+\boldsymbol{p}-\boldsymbol{q})}{N V}
\end{aligned}
$$

and

$$
\begin{aligned}
\left\langle j_{q}^{s L} \mid \rho_{k} j_{p}^{s L}\right\rangle & =\frac{1}{N}\left\langle\sum_{j}\left(\hat{\boldsymbol{q}} \cdot \boldsymbol{v}_{s}\right) e^{-i \boldsymbol{q} \cdot \boldsymbol{r}_{s}} e^{i \boldsymbol{k} \cdot \boldsymbol{r}_{j}}\left(\hat{\boldsymbol{p}} \cdot \boldsymbol{v}_{s}\right) e^{i \boldsymbol{p} \cdot \boldsymbol{r}_{s}}\right\rangle \\
& =\frac{T}{m} \frac{\delta(\boldsymbol{k}+\boldsymbol{p}-\boldsymbol{q})}{N V} S_{k} .
\end{aligned}
$$

The right vertex is determined by

$$
\left\langle\left\langle\rho_{k} \rho_{p}^{s} \mid \mathcal{L}_{+} j_{q}^{s L}\right\rangle\right\rangle=\left\langle\rho_{k} \rho_{p}^{s} \mid \mathcal{L}_{0} j_{q}^{s L}\right\rangle+\left\langle\rho_{k} \rho_{p}^{s} \mid \mathcal{T}_{+} j_{q}^{s L}\right\rangle .
$$


The free streaming contribution reads

$$
\left\langle\rho_{k} \rho_{p}^{s} \mid \mathcal{L}_{0} j_{q}^{s L}\right\rangle=q \frac{T}{m}\left\langle\rho_{k} \rho_{p}^{s} \mid \rho_{q}^{s}\right\rangle=q \frac{T}{m} \frac{\delta(\boldsymbol{k}+\boldsymbol{p}-\boldsymbol{q})}{N V} S_{k},
$$

while the collisional contribution differs from eq. F.31) above by a factor $\hat{\boldsymbol{q}} \exp \left(i \boldsymbol{q} \cdot \boldsymbol{r}_{s}\right)$ inside the average,

$$
\left\langle\rho_{k} \rho_{p}^{s} \mid \mathcal{T}_{+} j_{q}^{s L}\right\rangle=\frac{1+\epsilon}{2} \frac{\delta(\boldsymbol{k}+\boldsymbol{p}-\boldsymbol{q})}{N V} \frac{T}{m}\left(S_{k}-1\right) .
$$

\section{F.2.3 The Coherent Scattering Function}

The vertices are, again, a sum of two terms,

$$
\left\langle\left\langle j_{q}^{L} \mid \mathcal{L}_{+} \mathcal{Q}_{k} \rho_{p}\right\rangle\right\rangle=\left\langle\left\langle j_{q}^{L} \mid \mathcal{L}_{+} \rho_{k} \rho_{p}\right\rangle\right\rangle-N\left\langle\left\langle j_{q}^{L} \mid \mathcal{L}_{+} \rho_{q}\right\rangle\right\rangle\left\langle\rho_{q} \mid \rho_{k} \rho_{p}\right\rangle / S_{q}
$$

and

$$
\left\langle\left\langle\rho_{k} \rho_{p} \mid \mathcal{Q} \mathcal{L}_{+} j_{q}^{L}\right\rangle\right\rangle=\left\langle\left\langle\rho_{k} \rho_{p} \mid \mathcal{L}_{+} j_{q}^{L}\right\rangle\right\rangle-N\left\langle\rho_{k} \rho_{p} \mid \rho_{q}\right\rangle\left\langle\left\langle\rho_{q} \mid \mathcal{L}_{+} j_{q}^{L}\right\rangle\right\rangle / S_{q} .
$$

The left vertex only has a free streaming contribution,

$$
\left\langle j_{q}^{L} \mid \mathcal{L}_{+} \rho_{k} \rho_{p}\right\rangle=\left\langle j_{q}^{L} \mid \mathcal{L}_{0} \rho_{k} \rho_{p}\right\rangle=k\left\langle j_{q}^{L} \mid j_{k}^{L} \rho_{p}\right\rangle+p\left\langle j_{q}^{L} \mid \rho_{k} j_{p}^{L}\right\rangle .
$$

Invoking the definitions one finds

$$
\begin{aligned}
\left\langle j_{q}^{L} \mid j_{k}^{L} \rho_{p}\right\rangle & =\frac{1}{N^{3}}\left\langle\sum_{j, k, \ell}\left(\hat{\boldsymbol{q}} \cdot \boldsymbol{v}_{j}\right) e^{-i \boldsymbol{q} \cdot \boldsymbol{r}_{j}}\left(\hat{\boldsymbol{k}} \cdot \boldsymbol{v}_{k}\right) e^{i \boldsymbol{k} \cdot \boldsymbol{r}_{k}} e^{i \boldsymbol{p} \cdot \boldsymbol{r}_{\ell}}\right\rangle \\
& =\frac{1}{N^{3}}\left\langle\sum_{k, \ell}\left(\hat{\boldsymbol{q}} \cdot \boldsymbol{v}_{k}\right)^{2} e^{i(\boldsymbol{k}-\boldsymbol{q}) \cdot \boldsymbol{r}_{k}} e^{i \boldsymbol{p} \cdot \boldsymbol{r}_{\ell}}\right\rangle .
\end{aligned}
$$

The velocity integration yields a factor $T / m$ while the spatial integration essentially defines the structure factor again,

$$
\left\langle j_{q}^{L} \mid j_{k}^{L} \rho_{p}\right\rangle=\frac{T}{m} \frac{\delta(\boldsymbol{k}+\boldsymbol{p}-\boldsymbol{q})}{N^{2} V}\left(S_{k}-1\right) .
$$

For the free streaming contribution we get

$$
\left\langle\rho_{k} \rho_{p} \mid \mathcal{L}_{0} j_{q}^{L}\right\rangle=q \frac{T}{m}\left\langle\rho_{k} \rho_{p} \mid \rho_{q}\right\rangle=q \frac{\delta(\boldsymbol{k}+\boldsymbol{p}-\boldsymbol{q})}{N^{2} V} S^{(3)}(\boldsymbol{k}, \boldsymbol{p}) .
$$

The $\delta$-function, $\delta(\boldsymbol{k}+\boldsymbol{p}-\boldsymbol{q}) / V$, that imposes the momentum conservation constraint will be suppressed in the following to reduce clutter. 
The driving contribution vanishes for the same reason as in all the cases above. The collisional contribution on the other hand requires considerable work. In order to expand the different types of contributions to the following expression,

$$
\begin{aligned}
& \left\langle\rho_{k} \rho_{p} \mid \mathcal{T}_{+} j_{q}^{L}\right\rangle \\
& \quad=i \frac{1+\epsilon}{4 N} \frac{T}{m} \hat{\boldsymbol{q}} \cdot\left\langle\sum_{i} e^{-i \boldsymbol{k} \cdot \boldsymbol{r}_{i}} \sum_{j} e^{-i \boldsymbol{p} \cdot \boldsymbol{r}_{j}} \hat{\boldsymbol{r}}_{12} \delta\left(r_{12}-2 a\right)\left(e^{i \boldsymbol{q} \cdot \boldsymbol{r}_{2}}-e^{i \boldsymbol{q} \cdot \boldsymbol{r}_{1}}\right)\right\rangle
\end{aligned}
$$

I use a short hand notation for the average,

$$
\begin{aligned}
\langle i j \mid 12\rangle & =\langle 11 \mid 12\rangle+\langle 22 \mid 12\rangle+\langle 12 \mid 12\rangle+\langle 21 \mid 12\rangle \\
& +N(\langle 13 \mid 12\rangle+\langle 23 \mid 12\rangle+\langle 31 \mid 12\rangle+\langle 32 \mid 12\rangle+\langle 33 \mid 12\rangle) \\
& +N^{2}\langle 34 \mid 12\rangle .
\end{aligned}
$$

The number of unique terms can be further reduced by exploiting the symmetries to yield

$$
\langle i j \mid 12\rangle=2\langle 11 \mid 12\rangle+\langle 12 \mid 12\rangle+2 N\langle 13 \mid 12\rangle+N\langle 33 \mid 12\rangle+N^{2}\langle 34 \mid 12\rangle .
$$

Now I will treate this expression term by term. Fortunately, most of the terms eventually cancel each other and, thus, there is no need to calculate them explicitly.

$$
\langle 11 \mid 12\rangle=\left\langle e^{-i \boldsymbol{k} \cdot \boldsymbol{r}_{1}} e^{-i \boldsymbol{p} \cdot \boldsymbol{r}_{1}} \hat{\boldsymbol{r}}_{12} \delta\left(r_{12}-2 a\right)\left(e^{i \boldsymbol{q} \cdot \boldsymbol{r}_{2}}-e^{i \boldsymbol{q} \cdot \boldsymbol{r}_{1}}\right)\right\rangle
$$

Switching to relative coordinates,

$$
\langle 11 \mid 12\rangle=\left\langle e^{i(\boldsymbol{k}+\boldsymbol{p}-\boldsymbol{q}) \cdot \boldsymbol{r}_{1}} \hat{\boldsymbol{r}}_{12} \delta\left(r_{12}-2 a\right)\left(e^{i \boldsymbol{q} \cdot \boldsymbol{r}_{12}}-1\right)\right\rangle,
$$

one can extract the momentum conservation constraint and reduce the expression to the following standard form,

$$
\langle 11 \mid 12\rangle=\frac{\delta(\boldsymbol{k}+\boldsymbol{p}-\boldsymbol{q})}{V}\left\langle\hat{\boldsymbol{r}}_{12} \delta\left(r_{12}-2 a\right) e^{i \boldsymbol{q} \cdot \boldsymbol{r}_{12}}\right\rangle .
$$

Calculation of the second term proceeds by essentially the same steps,

$$
\langle 12 \mid 12\rangle=\left\langle e^{i \boldsymbol{k} \cdot \boldsymbol{r}_{1}} e^{i \boldsymbol{p} \cdot \boldsymbol{r}_{2}} \hat{\boldsymbol{r}}_{12} \delta\left(r_{12}-2 a\right)\left(e^{-i \boldsymbol{q} \cdot \boldsymbol{r}_{2}}-e^{-i \boldsymbol{q} \cdot \boldsymbol{r}_{1}}\right)\right\rangle .
$$

Introduction of relative coordinates,

$$
\langle 12 \mid 12\rangle=\left\langle e^{i(\boldsymbol{k}+\boldsymbol{p}-\boldsymbol{q}) \cdot \boldsymbol{r}_{2}} \hat{\boldsymbol{r}}_{12} \delta\left(r_{12}-2 a\right)\left(e^{i \boldsymbol{k} \cdot \boldsymbol{r}_{12}}-e^{i(\boldsymbol{k}-\boldsymbol{q}) \cdot \boldsymbol{r}_{12}}\right)\right\rangle,
$$


and reduction to the standard form,

$$
\langle 12 \mid 12\rangle=\frac{\delta(\boldsymbol{k}+\boldsymbol{p}-\boldsymbol{q})}{V}\left[\left\langle\hat{\boldsymbol{r}}_{12} \delta\left(r_{12}-2 a\right) e^{i \boldsymbol{k} \cdot \boldsymbol{r}_{12}}\right\rangle+\left\langle\hat{\boldsymbol{r}}_{21} \delta\left(r_{21}-2 a\right) e^{i \boldsymbol{p} \cdot \boldsymbol{r}_{21}}\right\rangle\right] .
$$

The first three particle term ,

$$
\langle 13 \mid 12\rangle=\left\langle e^{i \boldsymbol{k} \cdot \boldsymbol{r}_{1}} e^{i \boldsymbol{p} \cdot \boldsymbol{r}_{3}} \hat{\boldsymbol{r}}_{12} \delta\left(r_{12}-2 a\right)\left(e^{-i \boldsymbol{q} \cdot \boldsymbol{r}_{2}}-e^{-i \boldsymbol{q} \cdot \boldsymbol{r}_{1}}\right)\right\rangle,
$$

requires a little more work,

$$
\begin{aligned}
\langle 13 \mid 12\rangle & =\left\langle e^{i(\boldsymbol{k}+\boldsymbol{p}-\boldsymbol{q}) \cdot \boldsymbol{r}_{2}} e^{i \boldsymbol{k} \cdot \boldsymbol{r}_{12}} e^{i \boldsymbol{p} \cdot \boldsymbol{r}_{32}} \hat{\boldsymbol{r}}_{12} \delta\left(r_{12}-2 a\right)\right\rangle \\
& -\left\langle e^{i(\boldsymbol{k}-\boldsymbol{q}) \cdot \boldsymbol{r}_{1}} e^{i \boldsymbol{p} \cdot \boldsymbol{r}_{3}} \hat{\boldsymbol{r}}_{12} \delta\left(r_{12}-2 a\right)\right\rangle .
\end{aligned}
$$

The first term can be simplified by use of the BGY-relation again,

$$
\begin{aligned}
\left\langle e^{i(\boldsymbol{k}-\boldsymbol{q}) \cdot \boldsymbol{r}_{1}} e^{i \boldsymbol{p} \cdot \boldsymbol{r}_{3}} \hat{\boldsymbol{r}}_{12} \delta\left(r_{12}-2 a\right)\right\rangle & \\
& =\frac{\delta(\boldsymbol{k}+\boldsymbol{p}-\boldsymbol{q})}{N^{2} V}\left[i \boldsymbol{p}\left(S_{p}-1\right)+N\left\langle\hat{\boldsymbol{r}}_{12} \delta\left(r_{12}-2 a\right) e^{i \boldsymbol{p} \cdot \boldsymbol{r}_{12}}\right\rangle\right],
\end{aligned}
$$

while there is no need to evaluate the second term,

$$
\begin{aligned}
\langle 13 \mid 12\rangle=-\frac{\delta(\boldsymbol{k}+\boldsymbol{p}-\boldsymbol{q})}{N^{2} V}\left[i \boldsymbol{p}\left(S_{p}-1\right)\right. & +N\left\langle\hat{\boldsymbol{r}}_{12} \delta\left(r_{12}-2 a\right) e^{i \boldsymbol{p} \cdot \boldsymbol{r}_{12}}\right\rangle \\
& \left.-N^{2}\left\langle e^{i \boldsymbol{k} \cdot \boldsymbol{r}_{12}} e^{i \boldsymbol{p} \cdot \boldsymbol{r}_{32}} \hat{\boldsymbol{r}}_{12} \delta\left(r_{12}-2 a\right)\right\rangle\right]
\end{aligned}
$$

The same hold for the second three particle term,

$$
\langle 33 \mid 12\rangle=\left\langle e^{i \boldsymbol{k} \cdot \boldsymbol{r}_{3}} e^{i \boldsymbol{p} \cdot \boldsymbol{r}_{3}} \hat{\boldsymbol{r}}_{12} \delta\left(r_{12}-2 a\right)\left(e^{-i \boldsymbol{q} \cdot \boldsymbol{r}_{2}}-e^{-i \boldsymbol{q} \cdot \boldsymbol{r}_{1}}\right)\right\rangle .
$$

Use the YBG-relation,

$$
\langle 33 \mid 12\rangle=-2 \frac{\delta(\boldsymbol{k}+\boldsymbol{p}-\boldsymbol{q})}{N V^{2}}\left[\int d^{3} r e^{-i \boldsymbol{q} \cdot \boldsymbol{r}} \nabla g(r)-\int d^{3} r g(r) \delta(r-2 a) \hat{\boldsymbol{r}} e^{-i \boldsymbol{q} \cdot \boldsymbol{r}}\right],
$$

to bring it to the standard form,

$$
\langle 33 \mid 12\rangle=-2 \frac{\delta(\boldsymbol{k}+\boldsymbol{p}-\boldsymbol{q})}{N^{2} V}\left[i \boldsymbol{q}\left(S_{q}-1\right)+N\left\langle\hat{\boldsymbol{r}}_{12} \delta\left(r_{12}-2 a\right) e^{i \boldsymbol{q} \cdot \boldsymbol{r}_{12}}\right\rangle\right]
$$

The four particle term,

$$
\langle 34 \mid 12\rangle=\left\langle e^{i \boldsymbol{k} \cdot \boldsymbol{r}_{3}} e^{i \boldsymbol{p} \cdot \boldsymbol{r}_{4}} \hat{\boldsymbol{r}}_{12} \delta\left(r_{12}-2 a\right)\left(e^{-i \boldsymbol{q} \cdot \boldsymbol{r}_{2}}-e^{-i \boldsymbol{q} \cdot \boldsymbol{r}_{1}}\right)\right\rangle,
$$


can be reduced to three particle contributions by the help of the BGY-relation again,

$$
\begin{aligned}
\langle 34 \mid 12\rangle & =-\frac{2}{N V^{3}} \int d^{3} r_{2} d^{3} r_{3} d^{3} r_{4} e^{-i \boldsymbol{q} \cdot \boldsymbol{r}_{2}} e^{i \boldsymbol{k} \cdot \boldsymbol{r}_{3}} e^{i \boldsymbol{p} \cdot \boldsymbol{r}_{4}} \frac{\partial}{\partial \boldsymbol{r}_{2}} g_{3}\left(\boldsymbol{r}_{2}, \boldsymbol{r}_{3}, \boldsymbol{r}_{4}\right) \\
& +\frac{2}{N V^{3}} \int d^{3} r_{2} d^{3} r_{3} d^{3} r_{4} e^{-i \boldsymbol{q} \cdot \boldsymbol{r}_{2}} e^{i \boldsymbol{k} \cdot \boldsymbol{r}_{3}} e^{i \boldsymbol{p} \cdot \boldsymbol{r}_{4}} \hat{\boldsymbol{r}}_{23} \delta\left(r_{23}-2 a\right) g_{3}\left(\boldsymbol{r}_{2}, \boldsymbol{r}_{3}, \boldsymbol{r}_{4}\right) \\
& +\frac{2}{N V^{3}} \int d^{3} r_{2} d^{3} r_{3} d^{3} r_{4} e^{-i \boldsymbol{q} \cdot \boldsymbol{r}_{2}} e^{i \boldsymbol{k} \cdot \boldsymbol{r}_{3}} e^{i \boldsymbol{p} \cdot \boldsymbol{r}_{4}} \hat{\boldsymbol{r}}_{24} \delta\left(r_{24}-2 a\right) g_{3}\left(\boldsymbol{r}_{2}, \boldsymbol{r}_{3}, \boldsymbol{r}_{4}\right) .
\end{aligned}
$$

Extraction of the momentum conservation constraint,

$$
\begin{aligned}
\langle 34 \mid 12\rangle= & -\frac{2 i \boldsymbol{q}}{N V^{3}} \int d^{3} r_{2} d^{3} r_{23} d^{3} r_{24} e^{i(\boldsymbol{k}+\boldsymbol{p}-\boldsymbol{q}) \cdot \boldsymbol{r}_{2}} e^{-i \boldsymbol{k} \cdot \boldsymbol{r}_{23}} e^{-i \boldsymbol{p} \cdot \boldsymbol{r}_{24}} g_{3}\left(\boldsymbol{r}_{23}, \boldsymbol{r}_{24}\right) \\
& +\frac{2}{N V^{3}} \int d^{3} r_{2} d^{3} r_{23} d^{3} r_{24} e^{i(\boldsymbol{k}+\boldsymbol{p}-\boldsymbol{q}) \cdot \boldsymbol{r}_{2}} e^{-i \boldsymbol{k} \cdot \boldsymbol{r}_{23}} e^{-i \boldsymbol{p} \cdot \boldsymbol{r}_{24}} g_{3}\left(\boldsymbol{r}_{23}, \boldsymbol{r}_{24}\right) \hat{\boldsymbol{r}}_{23} \delta\left(r_{23}-2 a\right) \\
& +\frac{2}{N V^{3}} \int d^{3} r_{2} d^{3} r_{23} d^{3} r_{24} e^{i(\boldsymbol{k}+\boldsymbol{p}-\boldsymbol{q}) \cdot \boldsymbol{r}_{2}} e^{-i \boldsymbol{k} \cdot \boldsymbol{r}_{23}} e^{-i \boldsymbol{p} \cdot \boldsymbol{r}_{24}} g_{3}\left(\boldsymbol{r}_{23}, \boldsymbol{r}_{24}\right) \hat{\boldsymbol{r}}_{24} \delta\left(r_{24}-2 a\right),
\end{aligned}
$$

leaves us with three relatively simple terms,

$$
\begin{aligned}
\langle 34 \mid 12\rangle= & -\frac{2 i \boldsymbol{q}}{N^{3}} \frac{\delta(\boldsymbol{k}+\boldsymbol{p}-\boldsymbol{q})}{V}\left[S^{(3)}(\boldsymbol{k}, \boldsymbol{p})-S_{k}-S_{p}-S_{q}+2\right] \\
& -2 \frac{\delta(\boldsymbol{k}+\boldsymbol{p}-\boldsymbol{q})}{N V}\left\langle e^{i \boldsymbol{k} \cdot \boldsymbol{r}} e^{i \boldsymbol{p} \cdot \boldsymbol{R}} \hat{\boldsymbol{r}} \delta(r-2 a)\right\rangle \\
& -2 \frac{\delta(\boldsymbol{k}+\boldsymbol{p}-\boldsymbol{q})}{N V}\left\langle e^{i \boldsymbol{k} \cdot \boldsymbol{r}} e^{i \boldsymbol{p} \cdot \boldsymbol{R}} \hat{\boldsymbol{R}} \delta(R-2 a)\right\rangle .
\end{aligned}
$$

Adding up all the terms calculated above, most of them cancel as promised and we get

$$
\begin{aligned}
\langle i j \mid 12\rangle=-\frac{2 i}{N} \frac{\delta(\boldsymbol{k}+\boldsymbol{p}-\boldsymbol{q})}{V}\left[\boldsymbol{p}\left(S_{p}-1\right)\right. & +\boldsymbol{k}\left(S_{k}-1\right)+\boldsymbol{q}\left(S_{q}-1\right) \\
& \left.+\boldsymbol{q}\left(S^{(3)}(\boldsymbol{k}, \boldsymbol{p})-S_{k}-S_{p}-S_{q}+2\right)\right],
\end{aligned}
$$

which can be simplified further to yield

or

$$
\langle i j \mid 12\rangle=\frac{2 i}{N} \frac{\delta(\boldsymbol{k}+\boldsymbol{p}-\boldsymbol{q})}{V}\left[\boldsymbol{k} S_{p}+\boldsymbol{p} S_{k}-\boldsymbol{q} S^{(3)}(\boldsymbol{k}, \boldsymbol{p})\right]
$$

$$
\left\langle\rho_{k} \rho_{p} \mid \mathcal{T}_{+} j_{q}^{L}\right\rangle=\frac{1+\epsilon}{2 N^{2}} \frac{T}{m} \frac{\delta(\boldsymbol{k}+\boldsymbol{p}-\boldsymbol{q})}{V}\left[q S^{(3)}(\boldsymbol{k}, \boldsymbol{p})-(\hat{\boldsymbol{q}} \cdot \boldsymbol{k}) S_{p}-(\hat{\boldsymbol{q}} \cdot \boldsymbol{p}) S_{k}\right]
$$

and

$$
\begin{aligned}
& \left\langle\rho_{k} \rho_{p} \mid \mathcal{L}_{+} j_{q}^{L}\right\rangle \\
& \quad=-\frac{T}{m} \frac{\delta(\boldsymbol{k}+\boldsymbol{p}-\boldsymbol{q})}{N^{2} V}\left\{\frac{1+\epsilon}{2}\left[(\hat{\boldsymbol{q}} \cdot \boldsymbol{k}) S_{p}+(\hat{\boldsymbol{q}} \cdot \boldsymbol{p}) S_{k}\right]+\frac{1-\epsilon}{2} q S^{(3)}(\boldsymbol{k}, \boldsymbol{p})\right\}
\end{aligned}
$$

respectively. 


\section{F.3 Technicalities}

In this section I will collect some of the mostly technical aspects of the calculations.

To calculate a term like

$$
\left\langle\hat{\boldsymbol{r}} \delta(r-2 a) e^{i \boldsymbol{q} \cdot \boldsymbol{r}}\right\rangle=\int d^{3} r \hat{\boldsymbol{r}} \delta(r-2 a) g(r) e^{i \boldsymbol{q} \cdot \boldsymbol{r}},
$$

one switches to spherical coordinates,

$$
\left\langle\hat{\boldsymbol{r}} \delta(r-2 a) e^{i \boldsymbol{q} \cdot \boldsymbol{r}}\right\rangle=8 \pi a^{2} \chi \hat{\boldsymbol{q}} \int_{0}^{\pi} d \vartheta \sin \vartheta \cos \vartheta e^{2 i a q \cos \vartheta} .
$$

The remaining integral defines a spherical Bessel function [292],

$$
\left\langle\hat{\boldsymbol{r}} \delta(r-2 a) e^{i \boldsymbol{q} \cdot \boldsymbol{r}}\right\rangle=16 \pi i a^{2} \chi \hat{\boldsymbol{q}} j_{1}(2 a q) .
$$

To calculate a term like

$$
\begin{aligned}
N\left\langle\hat { \boldsymbol { r } } _ { 1 2 } \delta \left( r_{12}-\right.\right. & \left.2 a) e^{-i \boldsymbol{q} \cdot \boldsymbol{r}_{3}} e^{i \boldsymbol{q} \cdot \boldsymbol{r}_{2}}\right\rangle \\
& =\frac{n}{V^{2}} \int d^{3} r_{1} d^{3} r_{2} d^{3} r_{3} g_{3}\left(\boldsymbol{r}_{1}, \boldsymbol{r}_{2}, \boldsymbol{r}_{3}\right) \delta\left(r_{12}-2 a\right) \hat{\boldsymbol{r}}_{12} e^{-i \boldsymbol{q} \cdot \boldsymbol{r}_{3}} e^{i \boldsymbol{q} \cdot \boldsymbol{r}_{2}}
\end{aligned}
$$

one has to invoke the YBG-relation,

$$
\begin{aligned}
N\left\langle\hat{\boldsymbol{r}}_{12} \delta\left(r_{12}-2 a\right) e^{-i \boldsymbol{q} \cdot \boldsymbol{r}_{3}} e^{i \boldsymbol{q} \cdot \boldsymbol{r}_{2}}\right\rangle & =-\frac{1}{V^{2}} \int d^{3} r_{2} d^{3} r_{3} e^{-i \boldsymbol{q} \cdot \boldsymbol{r}_{3}} e^{i \boldsymbol{q} \cdot \boldsymbol{r}_{2}} \nabla_{23} g\left(r_{23}\right) \\
& +\frac{\chi}{V^{2}} \int d^{3} r_{2} d^{3} r_{3} \delta\left(r_{23}-2 a\right) \hat{\boldsymbol{r}}_{23} e^{-i \boldsymbol{q} \cdot \boldsymbol{r}_{3}} e^{i \boldsymbol{q} \cdot \boldsymbol{r}_{2}}
\end{aligned}
$$

The second term is rarely needed explicitly and is easier to recognize in the more compact notation. For the first term, switching to relative coordinates yields

$$
\begin{aligned}
N\left\langle\hat{\boldsymbol{r}}_{12} \delta\left(r_{12}-2 a\right) e^{-i \boldsymbol{q} \cdot \boldsymbol{r}_{3}} e^{i \boldsymbol{q} \cdot \boldsymbol{r}_{2}}\right\rangle= & -\frac{1}{V} \int d^{3} r e^{i \boldsymbol{q} \cdot \boldsymbol{r}} \nabla[g(r)-1] \\
& -\left\langle\hat{\boldsymbol{r}}_{12} \delta\left(r_{12}-2 a\right) e^{-i \boldsymbol{q} \cdot \boldsymbol{r}_{1}} e^{i \boldsymbol{q} \cdot \boldsymbol{r}_{2}}\right\rangle
\end{aligned}
$$

or

$$
N\left\langle\hat{\boldsymbol{r}}_{12} \delta\left(r_{12}-2 a\right) e^{-i \boldsymbol{q} \cdot \boldsymbol{r}_{3}} e^{i \boldsymbol{q} \cdot \boldsymbol{r}_{2}}\right\rangle=-\frac{1}{N}\left(S_{q}-1\right)-\left\langle\hat{\boldsymbol{r}}_{12} \delta\left(r_{12}-2 a\right) e^{-i \boldsymbol{q} \cdot \boldsymbol{r}_{1}} e^{i \boldsymbol{q} \cdot \boldsymbol{r}_{2}}\right\rangle .
$$

To calculate this third power of velocities,

$$
\begin{aligned}
\left\langle\left(\hat{\boldsymbol{q}} \cdot \boldsymbol{v}_{1}\right)\left(\hat{\boldsymbol{n}} \cdot \boldsymbol{v}_{12}\right)^{2} \Theta\left(-\hat{\boldsymbol{n}} \cdot \boldsymbol{v}_{12}\right)\right\rangle & =\hat{\boldsymbol{q}} \cdot\left\langle\boldsymbol{v}_{1}\left(\hat{\boldsymbol{n}} \cdot \boldsymbol{v}_{12}\right)^{2} \Theta\left(-\hat{\boldsymbol{n}} \cdot \boldsymbol{v}_{12}\right)\right\rangle \\
& =(\hat{\boldsymbol{q}} \cdot \hat{\boldsymbol{n}})\left\langle\left(\hat{\boldsymbol{n}} \cdot \boldsymbol{v}_{1}\right)\left(\hat{\boldsymbol{n}} \cdot \boldsymbol{v}_{12}\right)^{2} \Theta\left(-\hat{\boldsymbol{n}} \cdot \boldsymbol{v}_{12}\right)\right\rangle,
\end{aligned}
$$


it is best to switch to relative coordinates,

$$
\begin{aligned}
& \left\langle\left(\hat{\boldsymbol{n}} \cdot \boldsymbol{v}_{1}\right)\left(\hat{\boldsymbol{n}} \cdot \boldsymbol{v}_{12}\right)^{2} \Theta\left(-\hat{\boldsymbol{n}} \cdot \boldsymbol{v}_{12}\right)\right\rangle \\
& =2 \sqrt{2}\left(\frac{m}{2 \pi T}\right)^{3} \int d^{3} v d^{3} V\left[(\hat{\boldsymbol{n}} \cdot \boldsymbol{v})^{3}+(\hat{\boldsymbol{n}} \cdot \boldsymbol{v})^{2}(\hat{\boldsymbol{n}} \cdot \boldsymbol{V})\right] \Theta(-\hat{\boldsymbol{n}} \cdot \boldsymbol{v}) e^{-m\left(\boldsymbol{v}^{2}+V^{2}\right) / 2 T}
\end{aligned}
$$

The $\boldsymbol{V}$-integration yields only a trivial contribution while a transformation to spherical coordinates for $\boldsymbol{v}$,

$$
\begin{aligned}
&\left\langle\left(\hat{\boldsymbol{n}} \cdot \boldsymbol{v}_{1}\right)\left(\hat{\boldsymbol{n}} \cdot \boldsymbol{v}_{12}\right)^{2} \Theta\left(-\hat{\boldsymbol{n}} \cdot \boldsymbol{v}_{12}\right)\right\rangle \\
&=4 \sqrt{2} \pi\left(\frac{m}{2 \pi T}\right)^{3 / 2} \int_{0}^{\infty} d v \int_{\pi / 2}^{\pi} d \vartheta v^{5} \sin \vartheta \cos ^{3} \vartheta e^{-m v^{2} / 2 T}
\end{aligned}
$$

yields

$$
\left\langle\left(\hat{\boldsymbol{n}} \cdot \boldsymbol{v}_{1}\right)\left(\hat{\boldsymbol{n}} \cdot \boldsymbol{v}_{12}\right)^{2} \Theta\left(-\hat{\boldsymbol{n}} \cdot \boldsymbol{v}_{12}\right)\right\rangle=-\frac{4}{\sqrt{\pi}}\left(\frac{T}{m}\right)^{3 / 2}
$$

A term like

$$
\left\langle\left(\hat{\boldsymbol{q}} \cdot \hat{\boldsymbol{r}}_{12}\right)^{2} \delta\left(r_{12}-2 a\right) e^{i \boldsymbol{q} \cdot \boldsymbol{r}_{12}}\right\rangle=\frac{1}{V} \int d^{3} r g(r) \delta(r-2 a)(\hat{\boldsymbol{q}} \cdot \hat{\boldsymbol{r}})^{2} e^{i \boldsymbol{q} \cdot \boldsymbol{r}}
$$

can easily be reduced to Bessel functions using spherical coordinates,

$$
\left\langle\left(\hat{\boldsymbol{q}} \cdot \hat{\boldsymbol{r}}_{12}\right)^{2} e^{i \boldsymbol{q} \cdot \boldsymbol{r}_{12}} \delta\left(r_{12}-2 a\right)\right\rangle=\frac{8 \pi}{N} n a^{2} \chi \int_{0}^{\pi} d \vartheta \sin \vartheta \cos ^{2} \vartheta e^{2 i a q \cos \vartheta} .
$$

Using the identity [292],

$$
j_{2}(x)=\frac{3}{x} j_{1}(x)-j_{0}(x)
$$

for spherical Bessel functions, $j_{\nu}$, one finds

$$
\int_{0}^{\pi} d \vartheta \sin \vartheta \cos ^{2} \vartheta e^{i x \cos \vartheta}=2 j_{0}(x)-\frac{4}{x} j_{1}(x)=\frac{2}{3}\left[j_{0}(x)-2 j_{2}(x)\right]
$$

or

$$
\left\langle\left(\hat{\boldsymbol{q}} \cdot \hat{\boldsymbol{r}}_{12}\right)^{2} e^{i \boldsymbol{q} \cdot \boldsymbol{r}_{12}} \delta\left(r_{12}-2 a\right)\right\rangle=\frac{16 \pi}{3 N} n a^{2} \chi\left[j_{0}(2 a q)-2 j_{2}(2 a q)\right] .
$$





\section{Acknowledgment}

There are quite a few people that have contributed directly or indirectly to the completion of this thesis. It is a pleasure to thank all of them.

First and foremost I thank Prof. Dr. Annette Zippelius for a never ending stream of interesting subjects to work on, some of which made it into this thesis; for giving me the opportunity to work in her inspiring group and for the financial support that allowed me to fully concentrate on the scientific problems. I also thank Prof. Dr. Reiner Kree for his willingness to review this thesis on short notice and a very enjoyable lecture on "Mathematical Methods". I thank Dr. Matthias Sperl for his hospitality in Cologne. During that time I had a real breakthrough with my calculations. I also thank him for allowing me to use his MCT program and for countless fruitful discussions about MCT and granular matter. I thank PD Dr. Timo Aspelmeier for writing an excellent ED simulation program and Dr. Iraj Gholami for maintaining and extending it. I also learned a lot about physics and being a physicist from Timo, thank you for that.

Being trapped between the university and the MPI, I have to thank the secretaries: Katrin Glormann, Bärbel Lütge-Hampe, Giesela Mesecke, Gabriele Schubert, Ursula Glasneck, Germelina Meyer-Blank, Frank Rindermann for making all the bureaucratic processes as smooth as possible.

I thank Dr. Robert Peters and Dr. Jürgen Holm for keeping the cluster up and running. Burning so much CPU-time would have been much more challenging without you.

I thank all those that have read an earlier version of this thesis: Alice, Andrea, Martin, Robert, Stephan, Timo. Your input was extremely helpful.

During my time in the institute, I met a lot of very nice people. You provided me with the right mixture of coffee, tea and cake mixed with interesting discussions, scientific and other. Thank you. In particular, let me thank all my office mates, Wolfgang Barthel, Jens Birkholz, Stefan Wolfsheimer, Andrea Fiege, Taha Yasseri, Timo Aspelmeier, for providing a quiet but welcoming atmosphere and Alice von der Heydt, Martin Kiemes, Robert Peters and Stephan Ulrich for always having an open door.

There are even more people outside of the institute or outside of the physics community that supported me. Let me thank all of you. 



\section{Bibliography}

[1] D. J. Hornbaker, R. Albert, I. Albert, A. L. Barabási, and P. Schiffer. What keeps sandcastles standing? Nature, 387(6635):765, 1997.

[2] T. C. Halsey and A. J. Levine. How sandcastles fall. Phys. Rev. Lett., 80(14): 3141-3144, 1998.

[3] M. Scheel, R. Seemann, M. Brinkmann, M. Di Michiel, A. Sheppard, B. Breidenbach, and S. Herminghaus. Morphological clues to wet granular pile stability. Nature Mat., 7(3):189-193, 2008.

[4] T. Shinbrot and F. J. Muzzio. Nonequilibrium patterns in granular mixing and segregation. Phys. Today, 53:25, 2000.

[5] M. Ausloos, R. Lambiotte, K. Trojan, Z. Koza, et al. Granular Matter: a wonderful world of clusters in far-from-equilibrium systems. Physica A, 357(2): 337-349, 2005.

[6] I. S. Aranson and L. S. Tsimring. Patterns and collective behavior in granular media: Theoretical concepts. Rev. Mod Phys., 78(2):641-692, 2006.

[7] Labormikro. http://www.flickr.com/photos/labormikro/2809055850/, .

[8] Labormikro. http://www.flickr.com/photos/labormikro/3285208964/,

[9] Dominotic. http://www.flickr.com/photos/11061732@N07/5329813226/

[10] Ric Martin. http://www.flickr.com/photos/geckorem/2181849378/

[11] P. Richard, M. Nicodemi, R. Delannay, P. Ribière, and D. Bideau. Slow relaxation and compaction of granular systems. Nature Mat., 4(2):121-128, 2005 .

[12] R. M. Iverson. The physics of debris flows. Rev. Geophys., 35(3):245-296, 1997.

[13] B. T. Werner and B. Hallet. Numerical simulation of self-organized stone stripes. Nature, 361(6408):142, 1993. 
[14] R. D. Maladen, Y. Ding, C. Li, and D. I. Goldman. Undulatory swimming in sand: Subsurface locomotion of the sandfish lizard. Science, 325(5938):314, 2009 .

[15] T. Shimada, D. Kadau, T. Shinbrot, and H. J. Herrmann. Swimming in granular media. Phys. Rev. E, 80(2):020301, 2009.

[16] J. J. Lissauer. Planet formation. Ann. Rev. Astron. Astrophys., 31(1):129-172, 1993.

[17] P. Goldreich and S. Tremaine. The dynamics of planetary rings. Ann. Rev. Astron. Astrophys., 20:249-283, 1982.

[18] F. Spahn, J. M. Hertzsch, and N. V. Brilliantov. The role of particle collisions for the dynamics in planetary rings. Chaos, Solitons $\mathcal{E}$ Fractals, 5(10):1945-1964, 1995 .

[19] P. Goldreich and S. Tremaine. The velocity dispersion in Saturn's rings. Icarus, 34(2):227-239, 1978.

[20] H. M. Jaeger, S. R. Nagel, and R. P. Behringer. Granular solids, liquids, and gases. Rev. Mod. Phys., 68(4):1259-1273, 1996.

[21] H. M. Jaeger and S. R. Nagel. Physics of the granular state. Science, 255 (5051):1523, 1992.

[22] H. M. Jaeger, S. R. Nagel, and R. P. Behringer. The physics of granular materials. Phys. Today, 49:32, 1996.

[23] J. Rajchenbach. Granular flows. Adv. Phys., 49(2):229-256, 2000.

[24] S. Herminghaus. Dynamics of wet granular matter. Adv. Phys., 54(3):221-261, 2005.

[25] D. Champion, M. Le Meste, and D. Simatos. Towards an improved understanding of glass transition and relaxations in foods: molecular mobility in the glass transition range. Trends Food Sci. \& Tech., 11(2):41-55, 2000.

[26] M. Le Meste, D. Champion, G. Roudaut, G. Blond, and D. Simatos. Glass transition and food technology: a critical appraisal. J. Food Sci., 67(7):24442458, 2002.

[27] P. Bursac, G. Lenormand, B. Fabry, M. Oliver, D. A. Weitz, V. Viasnoff, J. P. Butler, and J. J. Fredberg. Cytoskeletal remodelling and slow dynamics in the living cell. Nature Mat., 4(7):557-561, 2005. 
[28] P. Sollich, F. Lequeux, P. Hébraud, and M.E. Cates. Rheology of soft glassy materials. Phys. Rev. Lett., 78(10):2020-2023, 1997.

[29] A. L. Greer. Metallic Glasses. Science, 267(5206):1947, 1995.

[30] R. Edgeworth, B. J. Dalton, and T. Parnell. The pitch drop experiment. Eur. J. Phys., 5:198, 1984.

[31] J. Mainstone, University of Queensland, and Saibo. GFDL, 2007.

[32] A. Cavagna. Supercooled liquids for pedestrians. Phys. Rep., 476(4-6):51-124, 2009 .

[33] I. M. Hodge. Physical aging in polymer glasses. Science, 267(5206):1945-1945, 1995.

[34] G. Biroli. A crash course on ageing. J. Stat. Mech., 2005:P05014, 2005.

[35] N. N. So Much More to Know .... Science, 309(5731):78b-102, 2005.

[36] C. A. Angell. Liquid fragility and the glass transition in water and aqueous solutions. Chem. Rev., 102(8):2627-2650, 2002.

[37] P. Jenniskens and D. F. Blake. Structural transitions in amorphous water ice and astrophysical implications. Science, 265(5173):753, 1994.

[38] M. E. Cates, J. P. Wittmer, J.-P. Bouchaud, and P. Claudin. Jamming, Force Chains, and Fragile Matter. Phys. Rev. Lett., 81(9):1841-1844, 1998.

[39] A. J. Liu and S. R. Nagel. Jamming is not just cool any more. Nature, 396 (6706):21-22, 1998.

[40] M. Pica Ciamarra, M. Nicodemi, and A. Coniglio. Recent results on the jamming phase diagram. Soft Matter, 6:2871, 2010.

[41] G. Marty and O. Dauchot. Subdiffusion and cage effect in a sheared granular material. Phys. Rev. Lett., 94(1):015701, 2005.

[42] A. R. Abate and D. J. Durian. Approach to jamming in an air-fluidized granular bed. Phys. Rev. E, 74(3):031308, 2006.

[43] D. I. Goldman and H. L. Swinney. Signatures of glass formation in a fluidized bed of hard spheres. Phys. Rev. Lett., 96(14):145702, 2006.

[44] P. M. Reis, R. A. Ingale, and M. D. Shattuck. Caging Dynamics in a Granular Fluid. Phys. Rev. Lett., 98(18):188301, 2007. 
[45] A. S. Keys, A. R. Abate, S. C. Glotzer, and D. J. Durian. Measurement of growing dynamical length scales and prediction of the jamming transition in a granular material. Nature physics, 3(4):260-264, 2007.

[46] K. Watanabe and H. Tanaka. Direct observation of medium-range crystalline order in granular liquids near the glass transition. Phys. Rev. Lett., 100(15): 158002, 2008.

[47] Corey S. O'Hern, Leonardo E. Silbert, Andrea J. Liu, and Sidney R. Nagel. Jamming at zero temperature and zero applied stress: The epitome of disorder. Phys. Rev. E, 68(1):011306, 2003.

[48] S. Chapman and T. G. Cowling. The Mathematical Theory of Non-Uniform Gases, volume 1. Cambridge University Press, 1960.

[49] L. D. Landau and E. M. Lifshitz. Fluid Mechanics, volume 6 of Course of Theoretical Physics. Butterworth-Heinemann, 2. edition, 1987.

[50] M. L. Tan and I. Goldhirsch. Rapid granular flows as mesoscopic systems. Phys. Rev. Lett., 81(14):3022-3025, 1998.

[51] I. Goldhirsch. Scales and kinetics of granular flows. Chaos, 9:659, 1999.

[52] S. McNamara. Hydrodynamic modes of a uniform granular medium. Phys. Fluids A, 5:3056, 1993.

[53] I. Goldhirsch and G. Zanetti. Clustering instability in dissipative gases. Phys. Rev. Lett., 70(11):1619-1622, 1993.

[54] A. Rosato, K. J. Strandburg, F. Prinz, and R. H. Swendsen. Why the Brazil nuts are on top: Size segregation of particulate matter by shaking. Phys. Rev. Lett., 58(10):1038-1040, 1987.

[55] M. Schröter, S. Ulrich, J. Kreft, J. B. Swift, and H. L. Swinney. Mechanisms in the size segregation of a binary granular mixture. Phys. Rev. E, 74(1):011307, 2006 .

[56] V. Garzó. Brazil-nut effect versus reverse Brazil-nut effect in a moderately dense granular fluid. Phys. Rev. E, 78(2):020301, 2008.

[57] L. P. Kadanoff. Built upon sand: Theoretical ideas inspired by granular flows. Rev. Mod. Phys., 71(1):435-444, 1999.

[58] I. Goldhirsch. Rapid Granular Flows. Annu. Rev. Fluid Mech., 35(1):267-293, 2003. 
[59] B. J. Alder and T. E. Wainwright. Velocity autocorrelations for hard spheres. Phys. Rev. Lett., 18(23):988-990, 1967.

[60] B. J. Alder and T. E. Wainwright. Decay of the velocity autocorrelation function. Phys. Rev. A, 1(1):18-21, 1970.

[61] T. E. Wainwright, B. J. Alder, and D. M. Gass. Decay of time correlations in two dimensions. Phys. Rev. A, 4(1):233-237, 1971.

[62] Y. Pomeau and P. Résibois. Time dependent correlation functions and modemode coupling theories. Phys. Rep., 19:63-139, 1975.

[63] J. R. Dorfman, T. R. Kirkpatrick, and J. V. Sengers. Generic long-range correlations in molecular fluids. Annu. Rev. Phys. Chem., 45(1):213-239, 1994.

[64] I. M. de Schepper, M. H. van Beyeren, and M. H. Ernst. The nonexistence of the linear diffusion equation beyond Fick's law. Physica, 75(1):1-36, 1974.

[65] J. R. Dorfman and E. G. D. Cohen. The virial expansion in equilibrium and nonequilibrium statistical mechanics. Intl. J. Quant. Chem., 22(S16):63-81, 1982.

[66] M. Otsuki and H. Hayakawa. Long-time tails for sheared fluids. J. Stat. Mech., 2009:L08003, 2009.

[67] A. Fiege, T. Aspelmeier, and A. Zippelius. Long-time tails and cage effect in driven granular fluids. Phys. Rev. Lett., 102(9):098001, 2009.

[68] N. W. Ashcroft and N. D. Mermin. Solid State Physics. Cengage Learning Services, 1976.

[69] J.-P. Hansen and I. R. McDonald. Theory of Simple Liquids. Academic Press, 3 edition, 2006.

[70] L. E. Busse. Temperature dependence of the structures of $\mathrm{As}_{2} \mathrm{Se}_{3}$ and $\mathrm{As}_{x} \mathrm{~S}_{1-x}$ glasses near the glass transition. Phys. Rev. B, 29(6):3639-3651, 1984.

[71] N. Goldenfeld. Lectures on Phase Transitions and the Renormalization Group. Addison Wesley, 1992.

[72] Per Bak, Chao Tang, and Kurt Wiesenfeld. Self-organized criticality: An explanation of the 1/f noise. Phys. Rev. Lett., 59(4):381-384, 1987.

[73] G. Peng and T. Ohta. Steady state properties of a driven granular medium. Phys. Rev. E, 58(4):4737-4746, 1998. 
[74] T. P. C. van Noije, M. H. Ernst, E. Trizac, and I. Pagonabarraga. Randomly driven granular fluids: Large-scale structure. Phys. Rev. E, 59(4):4326-4341, 1999.

[75] P. N. Pusey and W. van Megen. Phase behaviour of concentrated suspensions of nearly hard colloidal spheres. Nature, 320(6060):340-342, 1986.

[76] J. Kepler. On the six-cornered snowflake. Clarendon Press, Oxford, 1966. Trans.: C. Hardie.

[77] T. C. Hales. A proof of the Kepler conjecture. Ann. Math., 162:1065-1185, 2005.

[78] H. Löwen. Fun with Hard Spheres. In Statistical Physics and Spatial Statistics, volume 554 of Lecture Notes in Physics, page 295. Springer, Berlin, 2000.

[79] S. Sastry, T. M. Truskett, P. G. Debenedetti, S. Torquato, and F. H. Stillinger. Free volume in the hard sphere liquid. Mol. Phys., 95(2):289-298, 1998.

[80] W. G. Hoover and F. H. Ree. Melting transition and communal entropy for hard spheres. J. Chem. Phys., 49(8):3609, 1968.

[81] C. H. Mak. Large-scale simulations of the two-dimensional melting of hard disks. Phys. Rev. E, 73(6):065104, 2006.

[82] C. Song, P. Wang, and H. A. Makse. A phase diagram for jammed matter. Nature, 453(7195):629-632, 2008.

[83] D. Tabor. A simple theory of static and dynamic hardness. Proc. R. Soc. Lond. A, 192(1029):247-274, 1948.

[84] J. P. Dilley. Energy loss in collisions of icy spheres: Loss mechanism and size-mass dependence. Icarus, 105(1):225-234, 1993.

[85] T. Pöschel and V. Buchholtz. Molecular dynamics of arbitrarily shaped granular particles. J. Phys. I, 5(11):1431-1455, 1995.

[86] Y. Jiao, F. H. Stillinger, and S. Torquato. Optimal packings of superballs. Phys. Rev. E, 79(4):041309, 2009.

[87] G. Costantini, U. M. B. Marconi, G. Kalibaeva, and G. Ciccotti. The inelastic hard dimer gas: A nonspherical model for granular matter. J. Chem. Phys., 122:164505, 2005.

[88] M. Huthmann, T. Aspelmeier, and A. Zippelius. Granular cooling of hard needles. Phys. Rev. E, 60(1):654-659, 1999. 
[89] M. Otto, T. Aspelmeier, and A. Zippelius. Microscopic dynamics of thin hard rods. J. Chem. Phys., 124:154907, 2006.

[90] H. Gomart, J. Talbot, and P. Viot. Boltzmann equation for a granular capped rectangle in a thermalized bath of hard disks. Phys. Rev. E, 71(5):51306, 2005.

[91] H. Hertz. Über die Berührung fester elastischer Körper. J. Reine Angew. Math., 1882(92):156-171, 1882.

[92] L. Rayleigh. On the production of vibrations by forces of relatively long duration, with application to the theory of collisions. Phil. Mag., 11(3):283, 1906.

[93] F. Gerl and A. Zippelius. Coefficient of restitution for elastic disks. Phys. Rev. E, 59(2):2361-2372, 1999.

[94] T. Aspelmeier. Microscopic models of energy dissipation by internal degrees of freedom in particle collisions. PhD thesis, Georg-August-Universität Göttingen, 2000 .

[95] Y. H. Pao. Extension of the Hertz theory of impact to the viscoelastic case. J. Appl. Phys., 26:1083, 1955.

[96] N. V. Brilliantov, F. Spahn, J. M. Hertzsch, and T. Pöschel. Model for collisions in granular gases. Phys. Rev. E, 53(5):5382-5392, 1996.

[97] T. Schwager and T. Pöschel. Coefficient of restitution for viscoelastic spheres: The effect of delayed recovery. Phys. Rev. E, 78(5):51304, 2008.

[98] P. A. Cundall and O. D. L. Strack. A discrete numerical model for granular assemblies. Geotechnique, 29(1):47-65, 1979.

[99] P. K. Haff. Grain flow as a fluid-mechanical phenomenon. J. Fluid Mech., 134: 401-430, 1983.

[100] T. Aspelmeier, M. Huthmann, and A. Zippelius. Free Cooling of Particles with Rotational Degrees of Freedom. In Th. Pöschel and S. Luding, editors, Granular Gases, pages 31-58. Springer, 2001.

[101] S. F. Foerster, M. Y. Louge, H. Chang, and K. Allia. Measurements of the collision properties of small spheres. Phys. Fluids, 6:1108, 1994.

[102] A. Prevost, D. A. Egolf, and J. S. Urbach. Forcing and velocity correlations in a vibrated granular monolayer. Phys. Rev. Lett., 89(8):084301, 2002. 
[103] I. S. Aranson and J. S. Olafsen. Velocity fluctuations in electrostatically driven granular media. Phys. Rev. E, 66(6):061302, 2002.

[104] K. Kohlstedt, A. Snezhko, M. V. Sapozhnikov, I. S. Aranson, J. S. Olafsen, and E. Ben-Naim. Velocity distributions of granular gases with drag and with long-range interactions. Phys. Rev. Lett., 95(6):068001, 2005.

[105] C. C. Maaß, N. Isert, G. Maret, and C. M. Aegerter. Experimental investigation of the freely cooling granular gas. Phys. Rev. Lett., 100(24):248001, 2008.

[106] R. P. Ojha, P. A. Lemieux, P. K. Dixon, A. J. Liu, and D. J. Durian. Statistical mechanics of a gas-fluidized particle. Nature, 427(6974):521-523, 2004.

[107] A. R. Abate and D. J. Durian. Partition of energy for air-fluidized grains. Phys. Rev. E, 72(3):031305, 2005.

[108] M. Schröter, D. I. Goldman, and H. L. Swinney. Stationary state volume fluctuations in a granular medium. Phys. Rev. E, 71(3):030301, 2005.

[109] D. R. M. Williams and F. C. MacKintosh. Driven granular media in one dimension: Correlations and equation of state. Phys. Rev. E, 54(1):9-12, 1996.

[110] C. Bizon, M. D. Shattuck, J. B. Swift, and H. L. Swinney. Transport coefficients for granular media from molecular dynamics simulations. Phys. Rev. E, 60(4): 4340-4351, 1999.

[111] P. Espanol and P. Warren. Statistical mechanics of dissipative particle dynamics. EPL, 30:191-196, 1995.

[112] D. Forster, D. R. Nelson, and M. J. Stephen. Large-distance and long-time properties of a randomly stirred fluid. Phys. Rev. A, 16(2):732-749, 1977.

[113] N. F. Carnahan and K. E. Starling. Equation of state for nonattracting rigid spheres. J. Chem. Phys., 51:635, 1969.

[114] Y. Song, E. A. Mason, and R. M. Stratt. Why does the Carnahan-Starling equation work so well? J. Phys. Chem., 93(19):6916-6919, 1989.

[115] J. P. Boon and S. Yip. Molecular Hydrodynamics. Dover Publications, 1992.

[116] J. K. Percus and G. J. Yevick. Analysis of classical statistical mechanics by means of collective coordinates. Phys. Rev., 110(1):1-13, 1958.

[117] M. S. Wertheim. Exact solution of the Percus-Yevick integral equation for hard spheres. Phys. Rev. Lett., 10(8):321-323, 1963. 
[118] E. Thiele. Equation of state for hard spheres. J. Chem. Phys., 39:474, 1963.

[119] N. W. Ashcroft and J. Lekner. Structure and resistivity of liquid metals. Phys. Rev., 145(1):83-90, 1966.

[120] L. Verlet and J. J. Weis. Equilibrium theory of simple liquids. Phys. Rev. A, 5 (2):939-952, 1972.

[121] D. A. Head and H. Tanaka. Superdiffusive mass transport as a causal mechanism for large-scale structure formation. EPL, 91:40008, 2010.

[122] M. S. Green. Markoff Random Processes and the Statistical Mechanics of Time-Dependent Phenomena. II. Irreversible Processes in Fluids. J. Chem. Phys., 22:398, 1954.

[123] R. Kubo. Statistical-mechanical theory of irreversible processes. I. J. Phys. Soc. Jap., 12:570, 1957.

[124] K. Kawasaki and I. Oppenheim. Logarithmic term in the density expansion of transport coefficients. Phys. Rev, 139(6):1763-1768, 1965.

[125] J. D. Ferry. Viscoelastic properties of polymers. John Wiley \& Sons Inc, 1980.

[126] J. C. Maxwell. On the dynamic theory of gases. Phil. Trans. R. Soc, 157:49, 1867.

[127] D. Turnbull. Under what conditions can a glass be formed? Contemp. Phys., 10(5):473-488, 1969.

[128] W. Kauzmann. The Nature of the Glassy State and the Behavior of Liquids at Low Temperatures. Chem. Rev., 43(2):219-256, 1948.

[129] A. Cavagna, A. Attanasi, and J. Lorenzana. Viscoelasticity and metastability limit in supercooled liquids. Phys. Rev. Lett., 95(11):115702, 2005.

[130] J. Jäckle. Models of the glass transition. Rep. Prog. Phys., 49:171, 1986.

[131] C. A. Angell, P. H. Poole, and J. Shao. Glass-forming liquids, anomalous liquids, and polyamorphism in liquids and biopolymers. Nuovo Cimento D, 16 (8):993-1025, 1994.

[132] M. D. Ediger, C. A. Angell, and S. R. Nagel. Supercooled liquids and glasses. J. Phys. Chem, 100(31):13200-13212, 1996.

[133] P. G. Debenedetti and F. H. Stillinger. Supercooled liquids and the glass transition. Nature, 410(6825):259-267, 2001. 
[134] A. Inoue, T. Zhang, and T. Masumoto. Glass-forming ability of alloys. $J$. Non-Cryst. Solids, 156:473-480, 1993.

[135] S. L. Roux and P. Jund. Influence of the cooling rate on the glass transition temperature and the structural properties of glassy $\mathrm{GeS}_{2}$ : an ab initio molecular dynamics study. J. Phys.: Condensed Matt., 19:196102, 2007.

[136] C. A. Angell. Perspective on the glass transition. J. Phys. Chem. Solids, 49(8): 863-871, 1988.

[137] W. Kob and H. C. Andersen. Testing mode-coupling theory for a supercooled binary Lennard-Jones mixture I: The van Hove correlation function. Phys. Rev. E, 51(5):4626-4641, 1995.

[138] H. Vogel. Das Temperaturabhängigkeitsgesetz der Viskosität von Flüssigkeiten. Phys. Z, 22:645-646, 1921.

[139] G. S. Fulcher. Analysis of Recent Measurements of the Viscosity of Glasses. J. Americ. Ceramic Soc., 8(6):339-355, 1925.

[140] G. Tammann and W. Hesse. Die Abhängigkeit der Viscosität von der Temperatur bei unterkühlten Flüssigkeiten. Z. anorg. allg. Chem., 156(1):245-257, 1926.

[141] F. Stickel, E. W. Fischer, and R. Richert. Dynamics of glass-forming liquids. I. Temperature-derivative analysis of dielectric relaxation data. J. Chem. Phys., 102:6251, 1995.

[142] S. Arrhenius. Über die Reaktionsgeschwindigkeit bei der Inversion von Rohrzucker durch Säuren. Z. Phys. Chem., 4:226-248, 1889.

[143] R. Richert and C. A. Angell. Dynamics of glass-forming liquids. V. On the link between molecular dynamics and configurational entropy. J. Chem. Phys., 108:9016, 1998.

[144] W. Kob and H. C. Andersen. Testing mode-coupling theory for a supercooled binary Lennard-Jones mixture. II. Intermediate scattering function and dynamic susceptibility. Phys. Rev. E, 52(4):4134-4153, 1995.

[145] T. Castellani and A. Cavagna. Spin-glass theory for pedestrians. J. Stat. Mech., 2005:P05012, 2005.

[146] S. F. Edwards and P. W. Anderson. Theory of spin glasses. J. Phys. F, 5:965, 1975 . 
[147] P. Debye. Interferenz von Röntgenstrahlen und Wärmebewegung. Ann. Phys., 348(1):49-92, 1913.

[148] I. Waller. Zur Frage der Einwirkung der Wärmebewegung auf die Interferenz von Röntgenstrahlen. Z. Phys. A, 17(1):398-408, 1923.

[149] W. Götze and G. M. Vujičić. A comment on the Debye-Waller factor determination in undercooled liquids. Z. Phys. B, 76(2):175-178, 1989.

[150] G. U. Nienhaus and F. Parak. The Mössbauer effect and collective motions in glass-forming liquids and polymeric networks. Hyperfine Interact., 90(1): 243-264, 1994.

[151] G. Williams and D. C. Watts. Non-symmetrical dielectric relaxation behaviour arising from a simple empirical decay function. Trans. Faraday Soc., 66:80-85, 1970 .

[152] E. von Schweidler. Studien über die Anomalien im Verhalten der Dielektrika. Ann. Phys., 329(24):711-770, 1907.

[153] M. Fuchs, W. Götze, I. Hofacker, and A. Latz. Comments on the alpha-peak shapes for relaxation in supercooled liquids. J. Phys.: Condens. Matter, 3: $5047,1991$.

[154] T. Gleim, W. Kob, and K. Binder. How does the relaxation of a supercooled liquid depend on its microscopic dynamics? Phys. Rev. Lett., 81(20):4404-4407, 1998.

[155] T. Voigtmann, A. M. Puertas, and M. Fuchs. Tagged-particle dynamics in a hard-sphere system: Mode-coupling theory analysis. Phys. Rev. E, 70(6): 061506, 2004.

[156] G. Szamel and E. Flenner. Independence of the relaxation of a supercooled fluid from its microscopic dynamics: Need for yet another extension of the mode-coupling theory. EPL, 67:779, 2004.

[157] M. Goldstein. Viscous liquids and the glass transition: a potential energy barrier picture. J. Chem. Phys., 51:3728, 1969.

[158] A. P. Sokolov. The glass transition: general scenario and crossover temperature. J. Non-Cryst. Solids, 235:190-195, 1998.

[159] Y. Brumer and D. R. Reichman. Mean-field theory, mode-coupling theory, and the onset temperature in supercooled liquids. Phys. Rev. E, 69(4):041202, 2004 . 
[160] W. van Megen and P. N. Pusey. Dynamic light-scattering study of the glass transition in a colloidal suspension. Phys. Rev. A, 43(10):5429-5441, 1991.

[161] W. van Megen and S. M. Underwood. Dynamic-light-scattering study of glasses of hard colloidal spheres. Phys. Rev. E, 47(1):248-261, 1993.

[162] I. Volkov, M. Cieplak, J. Koplik, and J. R. Banavar. Molecular dynamics simulations of crystallization of hard spheres. Phys. Rev. E, 66(6):061401, 2002.

[163] R. Ni, S. Belli, R. van Roij, and M. Dijkstra. Glassy dynamics, spinodal fluctuations, and the kinetic limit of hard-rod nucleation, 2010.

[164] W. van Megen. Crystallisation and the glass transition in suspensions of hard colloidal spheres. Transp. Theory Stat. Phys., 24(6):1017-1051, 1995.

[165] J. M. Gordon, J. H. Gibbs, and P. D. Fleming. The hard sphere glass transition. J. Chem. Phys., 65:2771, 1976.

[166] L. V. Woodcock. Glass transition in the hard-sphere model. J. Chem. Soc. Faraday Trans. 2, 72:1667-1672, 1976.

[167] S. R. Williams, I. K. Snook, and W. van Megen. Molecular dynamics study of the stability of the hard sphere glass. Phys. Rev. E, 64(2):021506, 2001.

[168] E. Zaccarelli, C. Valeriani, E. Sanz, W. C. K. Poon, M. E. Cates, and P. N. Pusey. Crystallization of hard-sphere glasses. Phys. Rev. Lett., 103(13):135704, 2009.

[169] E. Sanz, Ch. Valeriani, E. Zaccarelli, W. C. K. Poon, P. N. Pusey, and M. E. Cates. Crystallization Mechanism of Hard Sphere Glasses. Phys. Rev. Lett., 106(21):215701, 2011.

[170] S. Auer and D. Frenkel. Suppression of crystal nucleation in polydisperse colloids due to increase of the surface free energy. Nature, 413(6857):711-713, 2001.

[171] P. N. Pusey, E. Zaccarelli, C. Valeriani, E. Sanz, W. C. K. Poon, and M. E. Cates. Hard spheres: crystallization and glass formation. Phil. Trans. R. Soc. A, 367(1909):4993, 2009.

[172] S. Martin, G. Bryant, and W. van Megen. Crystallization kinetics of polydisperse colloidal hard spheres: experimental evidence for local fractionation. Phys. Rev. E, 67(6):061405, 2003.

[173] M. Fasolo and P. Sollich. Fractionation effects in phase equilibria of polydisperse hard-sphere colloids. Phys. Rev. E, 70(4):041410, 2004. 
[174] W. Götze and L. Sjögren. $\beta$ relaxation at the glass transition of hard-spherical colloids. Phys. Rev. A, 43(10):5442-5448, 1991.

[175] H. Senff, W. Richtering, C. Norhausen, A. Weiss, and M. Ballauff. Rheology of a temperature sensitive core-shell latex. Langmuir, 15(1):102-106, 1999.

[176] P. A. Nommensen, M. H. G. Duits, D. van den Ende, and J. Mellema. Steady shear behavior of polymerically stabilized suspensions: Experiments and lubrication based modeling. Phys. Rev. E, 59(3):3147, 1999.

[177] P. Strating. Brownian dynamics simulation of a hard-sphere suspension. Phys. Rev. E, 59(2):2175, 1999.

[178] M. Fuchs and M. E. Cates. Theory of Nonlinear Rheology and Yielding of Dense Colloidal Suspensions. Phys. Rev. Lett., 89:248304, 2002.

[179] M. Fuchs and M. E. Cates. Schematic models for dynamic yielding of sheared colloidal glasses. Faraday Discuss., 123:267-286, 2003.

[180] M. Fuchs and M. E. Cates. Integration through transients for Brownian particles under steady shear. J. Phys: Condens. Matt., 17(20):1681, 2005.

[181] I. Gholami, A. Fiege, and A. Zippelius. Slow dynamics and precursors of the glass transition in granular fluids. art. arXiv:1106.3239, 2011.

[182] M. Fixman. Viscosity of critical mixtures. J. Chem. Phys., 36:310, 1962.

[183] L. P. Kadanoff and J. Swift. Transport coefficients near the liquid-gas critical point. Phys. Rev., 166(1):89-101, 1968.

[184] K. Kawasaki. Correlation-Function Approach to the Transport Coefficients near the Critical Point. I. Phys. Rev., 150(1):291-306, 1966.

[185] K. Kawasaki. Kinetic equations and time correlation functions of critical fluctuations. Ann. Phys., 61(1):1-56, 1970.

[186] K. Kawasaki. Mode coupling in critical phenomena and supercooled liquids. Transp. Theory Stat. Phys., 24(6):755-779, 1995.

[187] I. Oppenheim. Mode coupling and long time tails. Transp. Theory Stat. Phys., 24(6):781-798, 1995.

[188] S. P. Das. Mode-coupling theory and the glass transition in supercooled liquids. Rev. Mod. Phys., 76(3):785-851, 2004.

[189] M. Blume and J. Hubbard. Spin Correlation Functions at High Temperatures. Phys. Rev. B, 1(9):3815-3830, 1970. 
[190] E. Frey and F. Schwabl. Critical dynamics of magnets. Adv. Phys., 43(5): 577-683, 1994.

[191] R. H. Kraichnan. Decay of Isotropic Turbulence in the Direct-Interaction Approximation. Phys. Fluids, 7:1030, 1964.

[192] H. Goldstein, C. Poole, and J. Safko. Classical Mechanics. Addison Wesley, San Francisco, 3rd edition, 2002.

[193] V. I. Arnold. Mathematical methods of classical mechanics. Springer, 1989.

[194] B. O. Koopman. Hamiltonian Systems and Transformation in Hilbert Space. Proc. Natl. Acad. Sci., 17(5):315, 1931.

[195] B. L. Holian and D. J. Evans. Classical response theory in the Heisenberg picture. J. Chem. Phys., 83(7):3560-3566, 1985.

[196] D. J. Evans and G. P. Morriss. Statistical Mechanics of Nonequilibrium Liquids. ANU E Press, Canberra, 2 edition, 2007.

[197] M. Born and H. S. Green. A general kinetic theory of liquids. I. The molecular distribution functions. Proc. R. Soc. A, 188(1012):10, 1946.

[198] K. Kawasaki. Irreducible memory function for dissipative stochastic systems with detailed balance. Physica A, 215(1-2):61-74, 1995.

[199] M. H. Ernst, J. R. Dorfmann, W. R. Hoegy, and J. M. J. van Leeuwen. Hard-sphere dynamics and binary-collision operators. Physica, 45(1):127-146, 1969.

[200] A. R. Altenberger. On the calculation of the classical Liouville operator for the step-type interparticle interaction. Physica A, 80(1):46 - 62, 1975.

[201] J. E. Marsden. Generalized Hamiltonian mechanics a mathematical exposition of non-smooth dynamical systems and classical Hamiltonian mechanics. Arch. Rat. Mech. Ana., 28(5):323-361, 1968.

[202] S. Cordier, L. Pareschi, and G. Toscani. On a kinetic model for a simple market economy. J. Stat. Phys., 120(1):253-277, 2005.

[203] J. J. Brey, J. W. Dufty, and A. Santos. Dissipative dynamics for hard spheres. J. Stat. Phys., 87(5):1051-1066, 1997.

[204] M. Huthmann and A. Zippelius. Dynamics of inelastically colliding rough spheres: Relaxation of translational and rotational energy. Phys. Rev. E, 56 (6):6275-6278, 1997. 
[205] T. P. C. van Noije and M. H. Ernst. Velocity distributions in homogeneous granular fluids: The free and the heated case. Granular Matter, 1(2):57-64, 1998.

[206] J. M. Montanero and A. Santos. Computer simulation of uniformly heated granular fluids. Granul. Matter, 2(2):53-64, 2000.

[207] G. W. Baxter and J. S. Olafsen. Experimental evidence for molecular chaos in granular gases. Phys. Rev. Lett., 99(2):028001, 2007.

[208] M. H. Ernst, E. Trizac, and A. Barrat. The Boltzmann equation for driven systems of inelastic soft spheres. J. Stat. Phys., 124(2):549-586, 2006.

[209] S. H. Noskowicz, O. Bar-Lev, D. Serero, and I. Goldhirsch. Computer-aided kinetic theory and granular gases. EPL, 79:60001, 2007.

[210] C. Villani. Mathematics of granular materials. J. Stat. Phys., 124(2):781-822, 2006.

[211] P. Bak, C. Tang, and K. Wiesenfeld. Self-organized criticality. Phys. Rev. A, 38(1):364-374, 1988.

[212] T. Hwa and M. Kardar. Dissipative transport in open systems: An investigation of self-organized criticality. Phys. Rev. Lett., 62(16):1813-1816, 1989.

[213] G. Grinstein, D. H. Lee, and S. Sachdev. Conservation laws, anisotropy, and self-organized criticality in noisy nonequilibrium systems. Phys. Rev. Lett., 64 (16):1927-1930, 1990.

[214] G. Grinstein. Generic scale invariance in classical nonequilibrium systems (invited). J. Appl. Phys., 69:5441, 1991.

[215] J. Schofield and I. Oppenheim. The hydrodynamics of inelastic granular systems. Physica A, 196(2):209-240, 1993.

[216] J. J. Brey, J. W. Dufty, C. S. Kim, and A. Santos. Hydrodynamics for granular flow at low density. Phys. Rev. E, 58(4):4638, 1998.

[217] V. Garzó and J. W. Dufty. Dense fluid transport for inelastic hard spheres. Phys. Rev. E, 59(5):5895, 1999.

[218] J. W. Dufty. Fourier's Law for a Granular Fluid. J. Phys. Chem. C, 111(43): 15605, 2007.

[219] V. Garzó and J. M. Montanero. Transport coefficients of a heated granular gas. Physica A, 313(3-4):336-356, 2002. 
[220] R. F. Fox. Hydrodynamic fluctuation theories. J. Math. Phys., 19:1993, 1978.

[221] M. Toda, R. Kubo, and N. Saito. Statistical physics: Nonequilibrium statistical mechanics, volume 2 of Springer series in solid-state sciences. Springer, Berlin, 1991.

[222] P. Maynar, M. I. G. Soria, and E. Trizac. Fluctuating hydrodynamics for driven granular gases. EPJ ST, 179(1):123-139, 2009.

[223] S. R. de Groot and P. Mazur. Non-equilibrum Thermodynamics. Dover Publications, Inc., New York, 1984.

[224] B. D. Lubachevsky. How to simulate billiards and similar systems. J. Comp. Phys., 94(2):255-283, 1991.

[225] S. J. Cornell, M. R. Swift, and A. J. Bray. Inelastic collapse of a randomly forced particle. Phys. Rev. Lett., 81(6):1142-1145, 1998.

[226] T. W. Burkhardt and S. N. Kotsev. Equilibrium of a confined, randomly accelerated, inelastic particle: Is there inelastic collapse? Phys. Rev. E, 70(2): 026105, 2004.

[227] P. Deltour and J. L. Barrat. Quantitative study of a freely cooling granular medium. J. Phys. I, 7(1):137-151, 1997.

[228] S. Luding and S. McNamara. How to handle the inelastic collapse of a dissipative hard-sphere gas with the TC model. Granular Matter, 1(3):113-128, 1998.

[229] T. Aspelmeier. private communication.

[230] F. James. A review of pseudorandom number generators. Comp. Phys. Comm., 60(3):329-344, 1990.

[231] M. Matsumoto and T. Nishimura. Mersenne twister: a 623-dimensionally equidistributed uniform pseudo-random number generator. TOMACS, 8(1): 3-30, 1998.

[232] Eric Jones, Travis Oliphant, Pearu Peterson, et al. SciPy: Open source scientific tools for Python, 2001-. URL http://www.scipy.org/.

[233] C. Dellago and H. A. Posch. Kolmogorov-Sinai entropy and Lyapunov spectra of a hard-sphere gas. Physica A, 240(1-2):68-83, 1997.

[234] G. E. P. Box and Mervin E. Muller. A Note on the Generation of Random Normal Deviates. Ann. Math. Stat., 29(2):610-611, 1958. 
[235] K. Vollmayr-Lee, T. Aspelmeier, and A. Zippelius. Hydrodynamic correlation functions of a driven granular fluid in steady state. Phys. Rev. E, 83(1):011301, 2011.

[236] Y. Amarouchene and H. Kellay. Speed of sound from shock fronts in granular flows. Phys. Fluids, 18:031707, 2006.

[237] G. D'Anna, P. Mayor, A. Barrat, V. Loreto, and F. Nori. Observing brownian motion in vibration-fluidized granular matter. Nature, 424(6951):909-912, 2003.

[238] I. Goldhirsch, S. H. Noskowicz, and O. Bar-Lev. Nearly smooth granular gases. Phys. Rev. Lett., 95(6):068002, 2005.

[239] I. Goldhirsch, S. H. Noskowicz, and O. Bar-Lev. Hydrodynamics of Nearly Smooth Granular Gases. J. Phys. Chem. B, 109(45):21449-21470, 2005.

[240] O. R. Walton and R. L. Braun. Viscosity, granular-temperature, and stress calculations for shearing assemblies of inelastic, frictional disks. J. Rheol., 30 (5):949-980, 1986.

[241] A. Fiege, W. T. Kranz, T. Aspelmeier, and A. Zippelius. Large scale structure of driven granular fluids. in prepration.

[242] J. J. Brey, M. J. Ruiz-Montero, P. Maynar, and M. I. García de Soria. Hydrodynamic modes, Green-Kubo relations, and velocity correlations in dilute granular gases. J. Phys.: Condens. Matt., 17:S2489-S2502, 2005.

[243] J. Dufty, A. Baskaran, and J. J. Brey. Linear response for granular fluids. J. Stat. Mech., 2006:L08002, 2006.

[244] D. Forster. Hydrodynamic Fluctuations, Broken Symmetry, and Correlation Functions. W. A. Benjamin, 1975.

[245] R. Zwanzig. Ensemble method in the theory of irreversibility. J. Chem. Phys., $33: 1338,1960$.

[246] R. Zwanzig. Memory effects in irreversible thermodynamics. Phys. Rev., 124 (4):983-992, 1961.

[247] H. Mori. Transport, collective motion, and Brownian motion. Prog. Theo. Phys., 33:423-455, 1965.

[248] H. Mori. A continued-fraction representation of the time-correlation functions. Prog. Theor. Phys., 34:399-416, 1965. 
[249] H. C. Andersen. A Diagrammatic Formulation of the Kinetic Theory of Fluctuations in Equilibrium Classical Fluids. I. The Fluctuation Basis and the Cluster Properties of Associated Functions. J. Phys. Chem. B, 106(33): 8326-8337, 2002.

[250] H. C. Andersen. A diagrammatic formulation of the kinetic theory of fluctuations in equilibrium classical fluids. II. Equations of motion of the fluctuation fields and their diagrammatic solution. J. Phys. Chem. B, 107(37):10226-10233, 2003.

[251] H. C. Andersen. Diagrammatic formulation of the kinetic theory of fluctuations in equilibrium classical fluids. III. Cluster analysis of the renormalized interactions and a second diagrammatic representation of the correlation functions. $J$. Phys. Chem. B, 107(37):10234-10242, 2003.

[252] H. van Beijeren and M. H. Ernst. Kinetic theory of hard spheres. J. Stat. Phys., 21(2):125-167, 1979.

[253] M. von Smoluchowski. Über Brownsche Molekularbewegung unter Einwirkung äußerer Kräfte und deren Zusammenhang mit der verallgemeinerten Diffusionsgleichung. Ann. Phys., 353(48):1103-1112, 1915.

[254] B. Cichocki and W. Hess. On the memory function for the dynamic structure factor of interacting brownian particles. Physica. A, 141(2-3):475-488, 1987.

[255] S. J. Pitts and H. C. Andersen. The meaning of the irreducible memory function in stochastic theories of dynamics with detailed balance. J. Chem. Phys., 113: $3945,2000$.

[256] J. S. Thakur and J. Bosse. Glass transition of two-component liquids. II. The Lamb-Mössbauer factors. Phys. Rev. A, 43(8):4388, 1991.

[257] M. Fuchs, W. Götze, and M. R. Mayr. Asymptotic laws for tagged-particle motion in glassy systems. Phys. Rev. E, 58(3):3384-3399, 1998.

[258] U. Bengtzelius, W. Götze, and A. Sjölander. Dynamics of supercooled liquids and the glass transition. J. Phys. C, 17:5915-5934, 1984.

[259] M. H. Ernst, E. H. Hauge, and J. M. J. van Leeuwen. Asymptotic time behavior of correlation functions. I. Kinetic terms. Phys. Rev. A, 4(5):2055-2065, 1971.

[260] G. F. Mazenko. Fully Renormalized Kinetic Theory. II. Velocity Autocorrelation. Phys. Rev. A, 7(1):222-233, 1973.

[261] P. M. Furtado, G. F. Mazenko, and S. Yip. Effects of correlated collisions on atomic diffusion in a hard-sphere fluid. Phys. Rev. A, 14(2):869-876, 1976. 
[262] P. Resibois and J. L. Lebowitz. Approximate kinetic theory of hard-sphere fluids near equilibrium. I. Formal theory. J. Stat. Phys., 12(6):483-516, 1975.

[263] P. Resibois. Approximate kinetic theory of hard-sphere fluids near equilibrium: II. A quasihydrodynamic approximation for the velocity autocorrelation function. J. Stat. Phys., 13(5):393-426, 1975.

[264] L. Sjögren and A. Sjölander. Kinetic theory of self-motion in monatomic liquids. J. Phys. C, 12:4369-4392, 1979.

[265] L. Sjögren. Numerical results on the velocity correlation function in liquid argon and rubidium. J. Phys. C, 13:705-715, 1980.

[266] T. R. Kirkpatrick and J. C. Nieuwoudt. Mode-coupling theory of the large long-time tails in the stress-tensor autocorrelation function. Phys. Rev. A, 33 (4):2651-2657, 1986.

[267] T. R. Kirkpatrick and J. C. Nieuwoudt. Mode-coupling theory of the intermediate-time behavior of the velocity autocorrelation function. Phys. Rev. A, 33(4):2658-2662, 1986.

[268] M. Canales and J. Á. Padró. On the mode-coupling theory for the velocity autocorrelation functions of simple liquids. J. Phys.: Condens. Matter, 9:11009, 1997.

[269] J. Bosse, W. Götze, and A. Zippelius. Velocity-autocorrelation spectrum of simple classical liquids. Phys. Rev. A, 18(3):1214-1221, 1978.

[270] J. R. Dorfman and E. G. D. Cohen. Velocity-correlation functions in two and three dimensions: Low density. Phys. Rev. A, 6(2):776-790, 1972.

[271] E. P. Gross. Memory function for dressed particles. J. Stat. Phys., 11(6): 503-521, 1974.

[272] R. I. Cukier and J. R. Mehaffey. Kinetic theory of self-diffusion in a hard-sphere fluid. Phys. Rev. A, 18(3):1202-1213, 1978.

[273] H. H. H. Yuan and I. Oppenheim. Transport in two dimensions. I the selfdiffusion coefficient. Physica A, 90(1):1-20, 1978.

[274] G. F. Mazenko. Fully renormalized kinetic theory. III. Density fluctuations. Phys. Rev. A, 9(1):360-387, 1974.

[275] J. Bosse, W. Götze, and M. Lücke. Mode-coupling theory of simple classical liquids. Phys. Rev. A, 17(1):434-446, 1978. 
[276] L. Sjögren. Kinetic theory of current fluctuations in simple classical liquids. Phys. Rev. A, 22(6):2866-2882, 1980.

[277] L. Sjögren. Numerical results on the density fluctuations in liquid rubidium. Phys. Rev. A, 22(6):2883-2890, 1980.

[278] E. Leutheusser. Dynamics of a classical hard-sphere gas I. Formal theory. J. Phys. C, 15:2801-2826, 1982.

[279] E. Leutheusser. Dynamics of a classical hard-sphere gas. II. Numerical results. J. Phys. C, 15:2827, 1982.

[280] J. L. Barrat, W. Götze, and A. Latz. The liquid-glass transition of the hardsphere system. J. Phys.: Condens. Matter, 1:7163, 1989.

[281] W. Götze and L. Sjögren. The glass transition singularity. Z. Phys. B, 65(4): 415-427, 1987.

[282] C. D. Boley. Projection-operator approach to a renormalized kinetic theory. Phys. Rev. A, 11(1):328-339, 1975.

[283] M. Lindenfeld. Identity for memory operators in classical kinetic theory. Phys. Rev. A, 15(4):1801-1804, 1977.

[284] J. P. Bouchaud, L. Cugliandolo, J. Kurchan, and M. Mézard. Mode-coupling approximations, glass theory and disordered systems. Physica A, 226(3-4): 243-273, 1996.

[285] J. Jäckle and D. Sappelt. Test of analytical approximations for kinetic Ising models with sharp blocking transition. Physica A, 192(4):691-707, 1993.

[286] E. Leutheusser. Dynamical model of the liquid-glass transition. Phys. Rev. A, 29(5):2765-2773, 1984.

[287] W. Götze. Complex dynamics of glass-forming liquids: a mode-coupling theory. Oxford University Press, USA, 2009.

[288] G. Foffi, W. Götze, F. Sciortino, P. Tartaglia, and T. Voigtmann. $\alpha$-relaxation processes in binary hard-sphere mixtures. Phys. Rev. E, 69(1):011505, 2004.

[289] G. Wahnström and L. Sjögren. Incoherent scattering function in simple classical liquids. J. Phys. C, 15:401, 1982.

[290] J. Schofield and I. Oppenheim. Mode coupling and tagged particle correlation functions: the Stokes-Einstein law. Physica A, 187(1-2):210-242, 1992. 
[291] W. Götze and A. Zippelius. Incoherent scattering function $S_{s}(q, \omega)$ for classical liquids. Phys. Rev. A, 14(5):1842-1852, 1976.

[292] A. Jeffrey and D. Zwillinger, editors. Gradshteyn and Ryzhik's Table of Integrals, Series, and Products. Academic Press, 6th edition, 2000.

[293] M. H. Ernst and A. Weyland. Long time behaviour of the velocity autocorrelation function in a Lorentz gas. Phys. Lett. A, 34(1):39-40, 1971.

[294] W. T. Kranz, M. Sperl, and A. Zippelius. Glass Transition for Driven Granular Fluids. Phys. Rev. Lett., 104(22):225701, 2010.

[295] G. Szamel. Colloidal glass transition: Beyond mode-coupling theory. Phys. Rev. Lett., 90(22):228301, 2003.

[296] G. Szamel. Gaussian density fluctuations, mode coupling theory and all that. EPL, 65:498, 2004.

[297] J. Wu and J. Cao. High-order mode-coupling theory for the colloidal glass transition. Phys. Rev. Lett., 95(7):078301, 2005.

[298] Y. Uehara, Y. T. Lee, T. Ree, and F. H. Ree. Triplet distribution functions for hard spheres and hard disks. J. Chem. Phys., 70:1884, 1979.

[299] B. Bildstein and G. Kahl. Triplet correlation functions for hard-spheres: Computer simulation results. J. Chem. Phys., 100:5882, 1994.

[300] J. G. Kirkwood. Statistical mechanics of fluid mixtures. J. Chem. Phys., 3: 300, 1935.

[301] J. G. Kirkwood and E. M. Boggs. The radial distribution function in liquids. J. Chem. Phys., 10:394, 1942.

[302] A. Haffmans, I. M. de Schepper, J. P. J. Michels, and H. van Beijeren. Threeparticle equilibrium correlations in dense hard-sphere fluids. Phys. Rev. A, 37 (7):2698-2700, 1988.

[303] A. Singer. Maximum entropy formulation of the Kirkwood superposition approximation. J. Chem. Phys., 121:3657, 2004.

[304] T. Franosch, M. Fuchs, W. Götze, M. R. Mayr, and A. P. Singh. Asymptotic laws and preasymptotic correction formulas for the relaxation near glasstransition singularities. Phys. Rev. E, 55(6):7153, 1997.

[305] G. Nägele and J. Bergenholtz. Linear viscoelasticity of colloidal mixtures. J. Chem. Phys., 108:9893, 1998. 
[306] H. Hayakawa and M. Otsuki. Mode-coupling theory of sheared dense granular liquids. Prog. Theor. Phys., 119(3):381-402, 2008.

[307] J. Machta and I. Oppenheim. Mode coupling theory of hydrodynamics and steady state systems. Physica A, 112:361-392, 1982.

[308] J. Schofield, R. Lim, and I. Oppenheim. Mode coupling and generalized hydrodynamics. Physica A, 181(1-2):89-135, 1992.

[309] J. Schofield and I. Oppenheim. Mode coupling in nonequilibrium granular flow systems. Physica A, 204(1-4):555-605, 1994.

[310] J. L. Barrat and A. Latz. Mode coupling theory for the glass transition in a simple binary mixture. J. Phys.: Condens. Matt., 2:4289-4295, 1990.

[311] M. Fuchs and A. Latz. $\alpha$-relaxation in a supercooled binary mixture. Physica A, 201(1-3):1-13, 1993.

[312] R. Schmitz, J. W. Dufty, and P. De. Absence of a sharp glass transition in mode coupling theory. Phys. Rev. Lett., 71(13):2066-2069, 1993.

[313] C. Z. W. Liu and I. Oppenheim. Reexamination of the mode-coupling scheme for the glass transition. Physica A, 235(3-4):369-387, 1997.

[314] C. Z. W. Liu and I. Oppenheim. Microscopic theory for hopping transport in glass-forming liquids. Physica A, 247(1-4):183-195, 1997.

[315] M. Manno and I. Oppenheim. Microscopic theory for hopping transport in glass-forming liquids: mode coupling corrections. Physica A, 265(3-4):520-534, 1999.

[316] K. Kawasaki and K. Fuchizaki. An attempt to go beyond the current mode coupling theories of supercooled liquids and glass transitions. J. Non-Cryst. Solids, 235:57-65, 1998.

[317] L. Berthier, G. Biroli, J. P. Bouchaud, W. Kob, K. Miyazaki, and D. R. Reichman. Spontaneous and induced dynamic fluctuations in glass formers. I. General results and dependence on ensemble and dynamics. J. Chem. Phys, $126: 184503,2007$.

[318] L. Berthier, G. Biroli, J. P. Bouchaud, W. Kob, K. Miyazaki, and D. R. Reichman. Spontaneous and induced dynamic correlations in glass formers. II. Model calculations and comparison to numerical simulations. J. Chem. Phys., 126:184504, 2007. 
[319] A. Andreanov, G. Biroli, and J. P. Bouchaud. Mode coupling as a Landau theory of the glass transition. EPL, 88:16001, 2009.

[320] A. Ikeda and K. Miyazaki. Mode-Coupling Theory as a Mean-Field Description of the Glass Transition. Phys. Rev. Lett., 104(25):255704, 2010.

[321] S. P. Das. Glass transition and self-consistent mode-coupling theory. Phys. Rev. A, 42(10):6116-6124, 1990.

[322] B. Kim and K. Kawasaki. A fluctuation-dissipation relationship-preserving field theory for interacting Brownian particles: one-loop theory and mode coupling theory. J. Stat. Mech., 2004:1-60, 2008.

[323] H. W. Wyld. Formulation of the theory of turbulence in an incompressible fluid. Ann. Phys. NY, 14:143-165, 1961. 



\section{Curriculum Vitae}

Persönliche Daten

Name:

Wolf Till Kranz

Geburtstag, -ort:

Adresse:

Nationalität:

Ausbildung

$1986-1997$

$1997-2000$

Juni 2000

\section{Studium}

Oktober 2000

Oktober 2002

April 2003

Juli 2006

September 2006

deutsch
19. 06. 1979 in Mainz

Kasseler-Landstr. 19, 37081 Göttingen

Freie Waldorfschule Wiesbaden

Dr. Wilhelm Meyer-Gymnasium Braunschweig

Allgemeine Hochschulreife, Note: 1,3

Beginn Studium Physik an der Uni Augsburg

Vordiplom Physik

Fortsetzung Studium Physik an der Uni Göttingen

Diplom Physik, Note: sehr gut

Beginn Promotion Physik bei Prof. Dr. A. Zippelius

am Institut für Theor. Physik der Uni Göttingen

\section{Veröffentlichungen}

1. Kranz, W. T., M. Sperl and A. Zippelius, Glass Transition for Driven Granular Fluids, Phys. Rev. Lett. 104, 225701 (2010)

2. Kranz, W. T., N. V. Brilliantov, Th. Pöschel and A. Zippelius, Correlation of spin and velocity in the homogeneous cooling state of a granular gas of rough particles, EPJ ST 179, 91 (2009)

3. Uecker, H., W. T. Kranz, T. Aspelmeier and A. Zippelius, Partitioning of Energy in Highly Polydisperse Granular Gases, Phys. Rev. E 80, 041303 (2009)

4. Brilliantov, N. V., Th. Pöschel, W. T. Kranz and A. Zippelius, Translations and rotations are correlated in granular gases, Phys. Rev. Lett. 98, 28001 (2007) 